\title{
Supporting Information:
}

\section{Diastereo- and Enantioselective Synthesis of Structurally diverse Succinate, Butyrolactone, Trifluoromethyl Derivatives by Iridium Catalyzed Hydrogenation of Tetrasubstituted Olefins}

\author{
Sutthichat Kerdphon, ${ }^{\dagger[a]}$ Sudipta Ponra, ${ }^{[a]}$ Jianping Yang, ${ }^{\ddagger[a]}$ Haibo $W{ }^{[a]}$ Lars \\ Eriksson $^{[\mathrm{b}]}$ and Pher G. Andersson* ${ }^{[\mathrm{a}][\mathrm{c}]}$ \\ Email: Pher.Andersson@su.se \\ ${ }^{[a]}$ Department of Organic Chemistry, Stockholm University, Arrhenius Laboratory, 106 91, Stockholm, \\ Sweden; \\ ${ }^{[b]}$ Department of Materials and Environmental Chemistry, Stockholm University, Svante Arrhenius \\ väg16C, SE-10691, Stockholm, Sweden; \\ ${ }^{[c]}$ School of Chemistry and Physics, University of KwaZulu-Natal, Durban, South Africa.
}

\section{Table of contents}

1. General methods ........................................................... 1

2. General procedure for the preparation of $\alpha$-keto esters.......................... 2

3. General procedure for the preparation of tetrasubstituted olefins..................S3

4. Detailed optimization studies............................................ 16

5. General procedure for the hydrogenation reaction of the tetrasubstituted olefins......S19

6. Separation data and specific rotation for chiral compounds......................S30

7. General procedure for the preparation of bicyclic ligands......................... 35

8. General procedure for the preparation o the iridium complexes....................S37

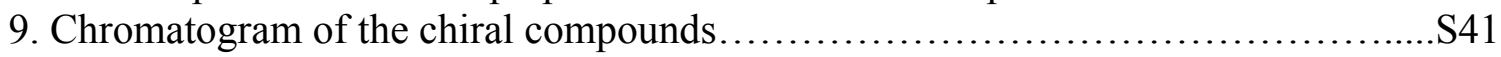

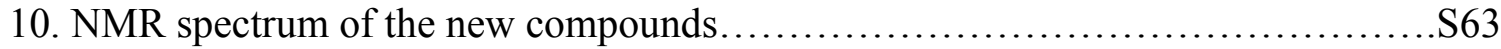

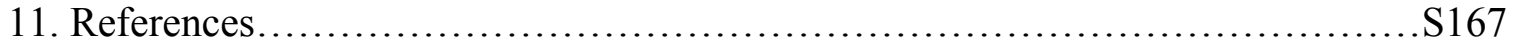

\section{General methods}

All glass vessels were dried in a vacuum oven $\left(160{ }^{\circ} \mathrm{C}\right)$ prior to use and cooled to room temperature under nitrogen. All the reactions were carried out using magnetic stirring under nitrogen atmosphere. THF was freshly dried over $\mathrm{Na} /$ benzophenone under nitrogen. $\mathrm{CH}_{2} \mathrm{Cl}_{2}$, pyridine and benzene were freshly distilled prior use from $\mathrm{CaH}_{2}$ under nitrogen. All reagents were purchased from commercial available sources and used directly or purified either by distillation or column chromatography. Thin-layer chromatography (TLC) was performed on aluminum plates coated with Kieselgel 60 (0.20 mm, UV 254) and visualized under ultraviolet light or either by staining with potassium permanganate solution or Hanessian's stain. ${ }^{1} \mathrm{HNMR}$ spectra was recorded at $400 \mathrm{MHz}$ in $\mathrm{CDCl}_{3}$ and referenced internally to the residual $\mathrm{CHCl}_{3}$ signal $(7.26 \mathrm{ppm})$.

${ }^{13} \mathrm{CNMR}$ spectra was recorded at $100 \mathrm{MHz}$ in $\mathrm{CDCl}_{3}$ and referenced to the central peak of $\mathrm{CDCl}_{3}(77.16 \mathrm{ppm})$. Chemical shifts were reported in ppm ( $\delta$ scale), and coupling constants $(J)$ were reported in Hertz $(\mathrm{Hz})$. High-resolution mass spectrometric (HRMS) data were obtained from the Bruker micro TOF-Q II instrument operating at ambient 
temperature. Optical rotation was recorded on a thermostated polarimeter using sodium lamp $(589 \mathrm{~nm})$ and a $10 \mathrm{~cm}$ cell. Enantiomeric excesses were determined using either GC-MS (30m columns, Helium gas carrier at $1 \mathrm{ml} / \mathrm{min}$, constant pressure), HPLC using chiral stationary phase. Racemic compounds were in all cases used for comparison.

\section{General procedure for preparation of $\alpha$-keto esters}

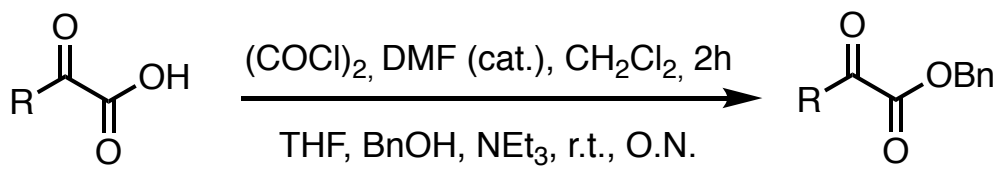

To a solution of the corresponding $\alpha$-keto acids ( $10.0 \mathrm{mmol}, 1.0$ equiv.) in $10.0 \mathrm{~mL}$ dry $\mathrm{DCM}(\mathrm{EtOH}$ free $)$ at $0^{\circ} \mathrm{C}$, oxalyl dichloride $(1 \mathrm{~mL}, 1.1$ equiv. $)$ and a drop of dry DMF were added sequentially. The mixture was then stirred for $2 \mathrm{~h}$ at room temperature, followed by the removal of the solvent and redundant oxalyl dichloride under reduced pressure. The residue was re-dissolved in dry THF $20 \mathrm{~mL}$ and benzyl alcohol $(1.2 \mathrm{~mL}$, 1.2 equiv.) was added. After drop-wise addition of $\mathrm{NEt}_{3}(4.2 \mathrm{~mL}, 30 \mathrm{mmol}, 3.0$ equiv.) the reaction mixture was stirred overnight at room temperature. After completion of the reaction, which was monitored by TLC, the reaction was quenched by addition of water. The phases were separated and the aqueous phase was extracted with diethyl ether $(3 \mathrm{x}$ $30 \mathrm{~mL}$ ). The combined extracts were dried over $\mathrm{Na}_{2} \mathrm{SO}_{4}$ and after removal of the solvent in vacuum, the crude $\alpha$-keto esters were obtained, which were purified by flash column chromatography over silica gel using diethyl ether, n-pentane mixture.

Various $\alpha$-keto esters were synthesized using this method and the characterizations of the new $\alpha$-keto esters are given below.

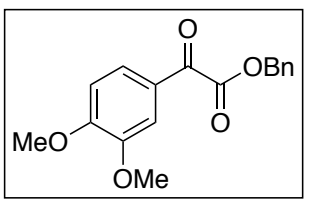

\section{Benzyl 2-(3,4-dimethoxyphenyl)-2-oxoacetate}

Yellow oil, 90\% yield. ${ }^{1} \mathrm{H}$ NMR $\left(\mathrm{CDCl}_{3}, 400 \mathrm{MHz}\right): \delta 7.58(\mathrm{ddd}, J=8.5,2.1,0.7 \mathrm{~Hz}$, $1 \mathrm{H}), 7.49-7.43(\mathrm{~m}, 3 \mathrm{H}), 7.42-7.35(\mathrm{~m}, 3 \mathrm{H}), 6.88(\mathrm{dd}, J=8.5,0.9 \mathrm{~Hz}, 1 \mathrm{H}), 5.40(\mathrm{~s}$, $2 \mathrm{H}), 3.95(\mathrm{~d}, J=1.3 \mathrm{~Hz}, 3 \mathrm{H}), 3.86(\mathrm{~d}, J=1.2 \mathrm{~Hz}, 3 \mathrm{H}) \cdot{ }^{13} \mathrm{C} \mathrm{NMR}\left(\mathrm{CDCl}_{3}, 100 \mathrm{MHz}\right): \delta$ $184.8,164.1,155.1,149.5,134.8,128.9,128.9$, 128.8, 126.2 , 125.7, 110.9, 110.4, 67.7, 56.4, 56.1. HRMS-ESI calcd for [M+Na] $]^{+}: 323.0895$, found: 323.0882 .

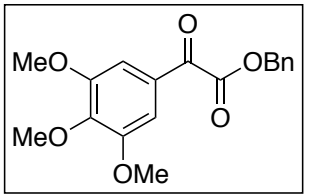

\section{Benzyl 2-oxo-2-(3,4,5-trimethoxyphenyl)acetate}

Yellow oil, $88 \%$ yield. ${ }^{1} \mathrm{H}$ NMR $\left(\mathrm{CDCl}_{3}, 400 \mathrm{MHz}\right): \delta 7.52-7.45(\mathrm{~m}, 2 \mathrm{H}), 7.43-7.34$ $(\mathrm{m}, 3 \mathrm{H}), 7.15(\mathrm{~s}, 2 \mathrm{H}), 5.42(\mathrm{~s}, 2 \mathrm{H}), 3.92(\mathrm{~s}, 3 \mathrm{H}), 3.78(\mathrm{~s}, 6 \mathrm{H}) \cdot{ }^{13} \mathrm{C} \mathrm{NMR}\left(\mathrm{CDCl}_{3}, 100\right.$ $\mathrm{MHz}): \delta 185.1,164.0,153.3,144.4,134.9,129.1,129.0,128.9,127.4,107.4,67.8,61.2$, 56.3. HRMS-ESI calcd for $[\mathrm{M}+\mathrm{Na}]^{+}: 353.1001$, found: 353.1003 . 


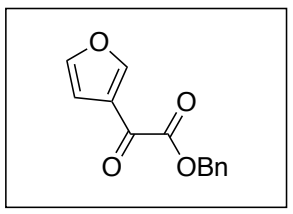

\section{Benzyl 2-(furan-3-yl)-2-oxoacetate}

Yellow oil, 84\% yield. ${ }^{1} \mathrm{H}$ NMR $\left(\mathrm{CDCl}_{3}, 400 \mathrm{MHz}\right): \delta 8.53-8.45(\mathrm{~m}, 1 \mathrm{H}), 7.51-7.32$ $(\mathrm{m}, 6 \mathrm{H}), 6.92-6.86(\mathrm{~m}, 1 \mathrm{H}), 5.36(\mathrm{~s}, 2 \mathrm{H}) .{ }^{13} \mathrm{C} \mathrm{NMR}\left(\mathrm{CDCl}_{3}, 100 \mathrm{MHz}\right): \delta 177.7,161.0$, $152.1,144.3,134.6,129.0,128.9,128.8,124.0,109.3,68.2$. HRMS-ESI calcd for $[\mathrm{M}+\mathrm{Na}]^{+}: 253.0471$, found: 253.0460 .

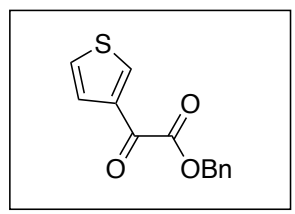

\section{Benzyl 2-oxo-2-(thiophen-3-yl)acetate}

Yellow oil, 90\% yield. ${ }^{1} \mathrm{H}$ NMR $\left(\mathrm{CDCl}_{3}, 400 \mathrm{MHz}\right): \delta 8.51-8.45(\mathrm{~m}, 1 \mathrm{H}), 7.69-7.64$ $(\mathrm{m}, 1 \mathrm{H}), 7.48-7.32(\mathrm{~m}, 6 \mathrm{H}), 5.39(\mathrm{~s}, 2 \mathrm{H}) .{ }^{13} \mathrm{C} \mathrm{NMR}\left(\mathrm{CDCl}_{3}, 100 \mathrm{MHz}\right): \delta 178.0,162.4$, 137.9, 137.7, 134.7, 128.9 (2C), 128.8, 128.0, 126.8, 68.1. HRMS-ESI calcd for $[\mathrm{M}+\mathrm{Na}]^{+}:$269.0243, found: 269.0253 .

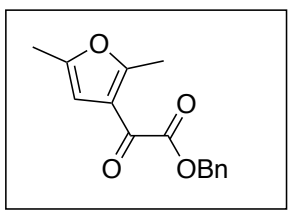

\section{Benzyl 2-(2,5-dimethylfuran-3-yl)-2-oxoacetate}

Yellow oil, $88 \%$ yield. ${ }^{1} \mathrm{H}$ NMR $\left(\mathrm{CDCl}_{3}, 400 \mathrm{MHz}\right): \delta 7.47-7.32(\mathrm{~m}, 5 \mathrm{H}), 6.42-6.38$ $(\mathrm{m}, 1 \mathrm{H}), 5.34(\mathrm{~s}, 2 \mathrm{H}), 2.51(\mathrm{~s}, 3 \mathrm{H}), 2.24(\mathrm{~s}, 3 \mathrm{H}) .{ }^{13} \mathrm{C} \mathrm{NMR}\left(\mathrm{CDCl}_{3}, 100 \mathrm{MHz}\right): \delta 180.1$, 163.0, 161.9, 151.0, 134.8, 128.8, 128.8, 128.8, 118.6, 106.3, 67.8, 14.7, 13.2. HRMSESI calcd for $[\mathrm{M}+\mathrm{Na}]^{+}: 281.0784$, found: 281.0791 .

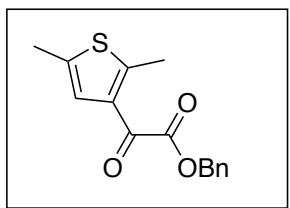

\section{Benzyl 2-(2,5-dimethylthiophen-3-yl)-2-oxoacetate}

Yellow oil, 82\% yield. ${ }^{1} \mathrm{H}$ NMR $\left(\mathrm{CDCl}_{3}, 400 \mathrm{MHz}\right): \delta 7.47-7.33(\mathrm{~m}, 5 \mathrm{H}), 7.02-6.98$ $(\mathrm{m}, 1 \mathrm{H}), 5.36(\mathrm{~s}, 2 \mathrm{H}), 2.66(\mathrm{~s}, 3 \mathrm{H}), 2.37(\mathrm{~s}, 3 \mathrm{H}) .{ }^{13} \mathrm{C} \mathrm{NMR}\left(\mathrm{CDCl}_{3}, 100 \mathrm{MHz}\right): \delta 180.4$, $164.0,152.9,136.1,134.8,131.4,128.9,128.8,128.8,126.9,67.7,16.1,15.0$. HRMSESI calcd for $[\mathrm{M}+\mathrm{Na}]^{+}: 297.0556$, found: 297.0558 .

\section{General procedure for preparation of tetrasubstituted olefins}

The tetrasubstituted olefins were prepared from a literature procedure either using the Wittig reaction $^{1}$ or by us the Horner-Wadsworth-Emmons reaction. ${ }^{2}$ 


\section{General procedure for the Wittig reaction}<smiles>[R]C(Br)C(=O)OCC</smiles>

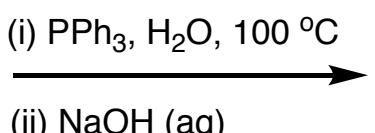

(ii) $\mathrm{NaOH}(\mathrm{aq})$<smiles>[R]C(=P)C(=O)OCC</smiles><smiles>[R]C(=O)C([R])=C([R])[R]</smiles>

To a solution of suitable 2-bromobutyrate $(12 \mathrm{mmol})$ in $\mathrm{H}_{2} \mathrm{O}(10 \mathrm{~mL})$ was added triphenylphosphine $(2.62 \mathrm{~g}, 10 \mathrm{mmol})$. The mixture was heated to reflux for one day. After cooling to room temperature, $\mathrm{CH}_{2} \mathrm{Cl}_{2}$ was used to extract the organics $(3 \mathrm{x} 10 \mathrm{~mL})$ and the aqueous layer was collected. The $\mathrm{pH}$ of the aqueous layer was adjusted to 12 13 with $10 \% \mathrm{NaOH}$ solution and phosphorene was extracted with $\mathrm{CH}_{2} \mathrm{Cl}_{2}(3 \times 10 \mathrm{~mL})$. After drying over $\mathrm{Na}_{2} \mathrm{SO}_{4}$, the organic layer was evaporated to give phosphorene as a foam-like yellow solid. This crude phosphorene was used in the next step without further purification.

To a stirring solution of phosphorane $(1 \mathrm{mmol})$ in toluene $(1.5 \mathrm{~mL})$ at $0{ }^{\circ} \mathrm{C}$ appropriate glyoxalate or ketone $(0.049 \mathrm{~mol})$ was added dropwise and the resulting mixture was stirred at reflux condition for 12 hours. After completion of the reaction, which was monitored by TLC, the reaction was quenched with water and was extracted with diethyl ether $(3 \times 10 \mathrm{~mL})$. The combined organic layers was dried over $\mathrm{Na}_{2} \mathrm{SO}_{4}$, filtered, and concentrated under reduced pressure. The crude product was subjected to flash chromatography-using n-pentane, $\mathrm{Et}_{2} \mathrm{O}$ mixture to afford the corresponding tetrasubstituted olefins.

Tetrasubstituted olefins $\mathbf{1 b}, \mathbf{2 g - 2} \mathbf{2}, \mathbf{2} \mathbf{p}-\mathbf{2} \mathbf{w}$ were synthesized following the Wittig reaction protocol.

\section{General procedure for the Horner-Wadsworth-Emmons reaction}<smiles>CCOC(=O)C(P)P(=O)(OCC)OCC</smiles>

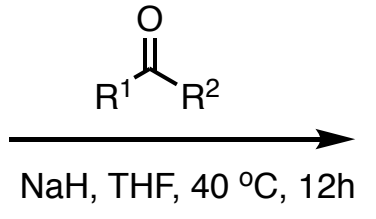<smiles>[R]C([R])=C([R])OCC</smiles>

To a stirred suspension of $\mathrm{NaH}$ ( $1 \mathrm{mmol}, 60 \%$ mineral dispersion) in anhydrous THF (2 $\mathrm{mL})$ at $0{ }^{\circ} \mathrm{C}$, the substituted triethyl phosphonoacetate $(1.02 \mathrm{mmol})$ was added dropwise. The reaction mixture was then heated to $40^{\circ} \mathrm{C}$ and stirred for 1 hour. After cooling again to $0{ }^{\circ} \mathrm{C}$, suitable glyoxalate or ketone $(0.049 \mathrm{~mol})$ was added dropwise and the resulting mixture was stirred at $40{ }^{\circ} \mathrm{C}$ for a further 12 hour. After completion of the reaction, monitored by TLC, the reaction was quenched with water. The reaction mixture was extracted with diethyl ether $(3 \times 10 \mathrm{~mL})$. The combined organic layers were dried over $\mathrm{Na}_{2} \mathrm{SO}_{4}$, filtered, and concentrated under reduced pressure. The crude product was purified by flash chromatography using n-pentane, $\mathrm{Et}_{2}$ Omixture to afford the desired tetrasubstituted olefins.

Tetrasubstituted olefins 1a, 1c-1f, 1n, 1o, 3a-3d and $\mathbf{5 f - 5 i}$ were prepared following this method. The assignments of $E$ or $Z$ configurations to the tetrasubstituted alkene substrates (1) were carried by the difference of $\delta$ values in ${ }^{1} \mathrm{H}-\mathrm{NMR}$ of $\mathrm{CH}_{2}$ of ethyl ester and comparing with similar known compounds. 


\section{General procedure for trifluoromethyl substituted tetra-substituted olefin synthesis ${ }^{3}$}

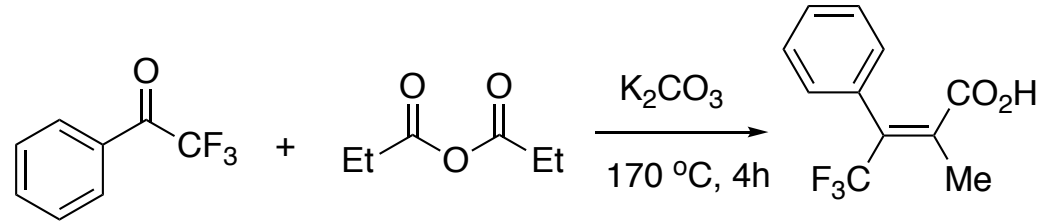

A mixture of $1.38 \mathrm{~mL}(10 \mathrm{mmol})$ of 2,2,2-trifluoroaceto-phenone, $1.86 \mathrm{~mL}(14.5 \mathrm{mmol})$ of propionic anhydride and $0.5 \mathrm{~g}(3.5 \mathrm{mmol})$ of anhydrous and powdered $\mathrm{K}_{2} \mathrm{CO}_{3}$ was heated in an oil bath at $170{ }^{\circ} \mathrm{C}$ for $4 \mathrm{~h}$. The mixture was cooled and $15 \mathrm{~mL}$ of $12 \% \mathrm{KOH}$ solution was added. The basic solution was extracted with $3 \times 10 \mathrm{~mL}$ diethyl ether and the aqueous phase was acidified with concentrated $\mathrm{HCl}$. The corresponding 2-methyl-3phenyl-4,4,4- trifluoro-2-butenoic acid was obtained as off-white solid and which was used without further purification for esterification.
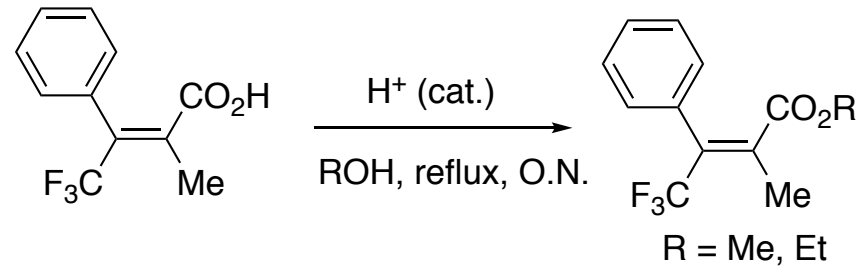

To 2-methyl-3-phenyl-4,4,4-trifluoro-2-butenoic acid $(5 \mathrm{mmol})$ appropriate alcohol (5 $\mathrm{mL})$ was added followed by few drops of conc. $\mathrm{H}_{2} \mathrm{SO}_{4}(0.1 \mathrm{~mL})$ and the resulting mixture was stirred at reflux condition for over night. After completion of the reaction, monitored by TLC, alcohol was removed under reduced pressure and the reaction was quenched with water. The reaction mixture was extracted with diethyl ether $(3 \times 10 \mathrm{~mL})$. The combined organic layers were dried over $\mathrm{Na}_{2} \mathrm{SO}_{4}$, filtered, and concentrated under reduced pressure. The crude product was purified by flash chromatography using npentane, $\mathrm{Et}_{2} \mathrm{O}$ mixture to afford the desired tetrasubstituted olefins $\mathbf{5 a}$ or $\mathbf{5 d}$.

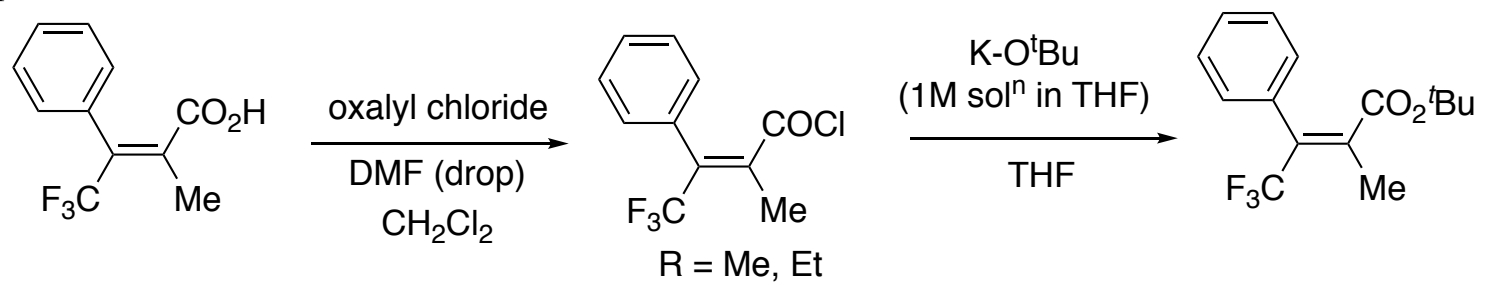

Oxalyl chloride (1.05 equiv.) was added dropwise to the 2-methyl-3-phenyl-4,4,4trifluoro-2-butenoic acid (1 equiv) in $\mathrm{CH}_{2} \mathrm{Cl}_{2}(10 \mathrm{~mL} / \mathrm{g})$, followed by a drop of DMF. Once all the acid had been consumed (TLC analysis of an aliquot after a MeOH quench), the solvent was removed in vacuo. THF $(10 \mathrm{~mL} / \mathrm{g})$ was added and the mixture cooled to 0 ${ }^{\circ} \mathrm{C}$. K-O ${ }^{t} \mathrm{Bu}\left(1 \mathrm{M}\right.$ solution in THF, 1.2 equiv) was added. After 10 minutes at $0{ }^{\circ} \mathrm{C}$, the ice bath was removed and the reaction mixture stirred at room temperature for over night. After completion of the reaction, monitored by TLC, the reaction mixture was diluted with diethyl ether $(30 \mathrm{~mL} / \mathrm{g})$ and washed with water $(30 \mathrm{~mL} / \mathrm{g})$. The aqueous phase was extracted with diethyl ether $(3 \times 30 \mathrm{~mL} / \mathrm{g})$. The combined organic layers were dried over $\mathrm{Na}_{2} \mathrm{SO}_{4}$, filtered, and concentrated under reduced pressure. The crude product was 
purified by flash chromatography using n-pentane, $\mathrm{Et}_{2} \mathrm{O}$ mixture to afford the desired tetrasubstituted olefins.

Tetrasubstituted olefin $\mathbf{5 c}$ was prepared following this method.

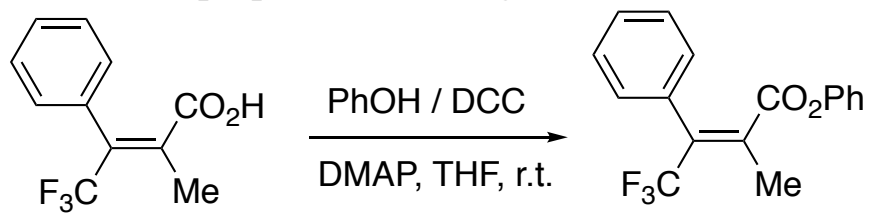

To a mixture of 2-methyl-3-phenyl-4,4,4- trifluoro-2-butenoic acid (1 equiv) and phenol (1.2 equiv) in THF (10 mL), DCC (1.2 equiv) and DMAP (catalytic amount) was added. The resulting mixture was stirred at room temperature for over night. After completion of the reaction, monitored by TLC, THF was removed under reduced pressure and the reaction was quenched with water. The reaction mixture was extracted with diethyl ether $(3 \times 10 \mathrm{~mL})$. The combined organic layers were dried over $\mathrm{Na}_{2} \mathrm{SO}_{4}$, filtered, and concentrated under reduced pressure. The crude product was purified by flash chromatography using n-pentane, $\mathrm{Et}_{2} \mathrm{O}$ mixture to afford the desired tetrasubstituted olefins.

Tetrasubstituted olefin 5e was prepared following this method. The assignments of $E$ or $Z$ configurations to the tetrasubstituted alkene substrates (5) were carried by the difference of ${ }^{19}$ F-NMR and comparing with similar known compounds.

The characterizations of the new tetrasubstituted olefins are given below.

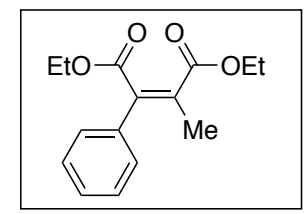

\section{Diethyl 2-methyl-3-phenylmaleate (1a)}

Yellow oil, 40\% yield. ${ }^{1} \mathrm{H}$ NMR $\left(\mathrm{CDCl}_{3}, 400 \mathrm{MHz}\right): \delta 7.44-7.33(\mathrm{~m}, 3 \mathrm{H}), 7.32-7.28$ $(\mathrm{m}, 2 \mathrm{H}), 4.28(\mathrm{q}, 2 \mathrm{H}), 4.22(\mathrm{q}, J=7.3 \mathrm{~Hz}, 2 \mathrm{H}), 1.90(\mathrm{~s}, 3 \mathrm{H}), 1.33(\mathrm{t}, J=7.1 \mathrm{~Hz}, 3 \mathrm{H}), 1.26$ $(\mathrm{t}, J=7.1 \mathrm{~Hz}, 3 \mathrm{H}) .{ }^{13} \mathrm{C} \mathrm{NMR}\left(\mathrm{CDCl}_{3}, 100 \mathrm{MHz}\right): \delta 168.3,168.3,140.2,135.0132 .9$, $128.8,128.6,61.5(2 \mathrm{C}), 16.5,14.2,14.1$. HRMS-ESI calcd for $[\mathrm{M}+\mathrm{Na}]^{+}: 285.1097$, found: 285.1107 .

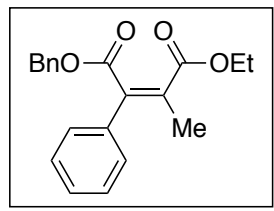

\section{1-Benzyl 4-ethyl 3-methyl-2-phenylmaleate (1b)}

Colourless oil, 55\% yield. ${ }^{1} \mathrm{H}$ NMR $\left(\mathrm{CDCl}_{3}, 400 \mathrm{MHz}\right): \delta 7.41-7.33(\mathrm{~m}, 3 \mathrm{H}), 7.30$ (ttd, $J=5.3,4.0,3.5,1.9 \mathrm{~Hz}, 7 \mathrm{H}), 5.20(\mathrm{~s}, 2 \mathrm{H}), 4.18(\mathrm{q}, J=7.1 \mathrm{~Hz}, 2 \mathrm{H}), 1.90(\mathrm{~s}, 3 \mathrm{H}), 1.27(\mathrm{t}$, $J=7.1 \mathrm{~Hz}, 3 \mathrm{H}) .{ }^{13} \mathrm{C} \mathrm{NMR}\left(\mathrm{CDCl}_{3}, 100 \mathrm{MHz}\right): \delta 168.3,168.0,139.6,135.7,134.9,133.6$, $128.8,128.6,128.6,128.3,67.3,61.5,16.6,14.2$. HRMS-ESI calcd for $[\mathrm{M}+\mathrm{Na}]^{+}$: 347.1254, found: 347.1271 . 


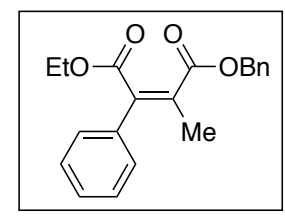

\section{1-Benzyl 4-ethyl 2-methyl-3-phenylmaleate (1c)}

Colourless oil, $20 \%$ yield. ${ }^{1} \mathrm{H}$ NMR $\left(\mathrm{CDCl}_{3}, 400 \mathrm{MHz}\right): \delta 7.41-7.34(\mathrm{~m}, 8 \mathrm{H}), 7.31(\mathrm{~d}, J$ $=1.9 \mathrm{~Hz}, 1 \mathrm{H}), 7.29(\mathrm{t}, J=1.7 \mathrm{~Hz}, 1 \mathrm{H}), 5.25(\mathrm{~s}, 2 \mathrm{H}), 4.07(\mathrm{q}, J=7.1 \mathrm{~Hz}, 2 \mathrm{H}), 1.92(\mathrm{~s}$, $3 \mathrm{H}), 1.17(\mathrm{t}, J=7.1 \mathrm{~Hz}, 3 \mathrm{H}) .{ }^{13} \mathrm{C} \mathrm{NMR}\left(\mathrm{CDCl}_{3}, 100 \mathrm{MHz}\right): \delta 168.2,168.1,140.9,135.6$, $134.9,132.2,128.7,128.6,128.6,128.6,128.5,128.3,67.4,61.5,16.5,14.0$. HRMS-ESI calcd for $[\mathrm{M}+\mathrm{Na}]^{+}: 347.1259$, found: 347.1254 .

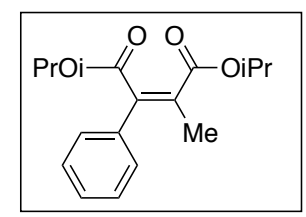

\section{Diisopropyl 2-methyl-3-phenylmaleate}

Colorless oil, $20 \%$ yield. ${ }^{1} \mathrm{H}$ NMR $\left(\mathrm{CDCl}_{3}, 400 \mathrm{MHz}\right): \delta 7.46-7.14(\mathrm{~m}, 5 \mathrm{H}), 5.19-5.05$ $(\mathrm{m}, 2 \mathrm{H}), 1.88(\mathrm{~s}, 3 \mathrm{H}), 1.32(\mathrm{~d}, J=6.3 \mathrm{~Hz}, 6 \mathrm{H}), 1.23(\mathrm{~d}, J=6.3 \mathrm{~Hz}, 6 \mathrm{H}) .{ }^{13} \mathrm{C} \mathrm{NMR}$ $\left(\mathrm{CDCl}_{3}, 100 \mathrm{MHz}\right): \delta 167.8,167.74,2,140.5,135.2,132.5,128.7,128.5,128.4,67.0$, 68.9, 21.8, 21.7, 16.5. HRMS-ESI calcd for [M+Na] $]^{+}: 313.1410$, found: 313.1422 .

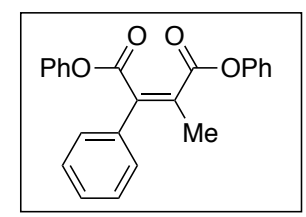

\section{Diphenyl 2-methyl-3-phenylmaleate}

Yellow oil, $23 \%$ yield. ${ }^{1} \mathrm{H}$ NMR $\left(\mathrm{CDCl}_{3}, 400 \mathrm{MHz}\right): \delta 7.51-7.46(\mathrm{~m}, 4 \mathrm{H}), 7.46-7.38$ $(\mathrm{m}, 3 \mathrm{H}), 7.35-7.29(\mathrm{~m}, 2 \mathrm{H}), 7.26(\mathrm{t}, J=3.6 \mathrm{~Hz}, 1 \mathrm{H}), 7.23-7.17(\mathrm{~m}, 3 \mathrm{H}), 7.09-7.07$ $(\mathrm{m}, 2 \mathrm{H}), 2.15(\mathrm{~d}, J=1.0 \mathrm{~Hz}, 3 \mathrm{H}) .{ }^{13} \mathrm{C} \mathrm{NMR}\left(\mathrm{CDCl}_{3}, 100 \mathrm{MHz}\right): \delta 166.6,166.4,150.7$, $150.7,141.0,134.4,133.4,129.7,129.5,129.1,128.9,126.3,126.2,121.7,121.6,16.8$. HRMS-ESI calcd for [M+Na] $]^{+}: 381.1097$, found: 381.1082 .

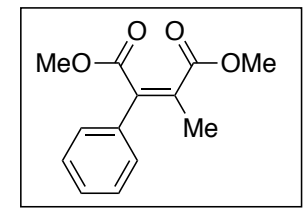

\section{Dimethyl 2-methyl-3-phenylmaleate}

Colourless oil, $31 \%$ yield. ${ }^{1} \mathrm{H}$ NMR $\left(\mathrm{CDCl}_{3}, 400 \mathrm{MHz}\right): \delta 7.43-7.35(\mathrm{~m}, 3 \mathrm{H}), 7.32-$ $7.27(\mathrm{~m}, 2 \mathrm{H}), 3.83(\mathrm{~s}, 3 \mathrm{H}), 3.76(\mathrm{~s}, 3 \mathrm{H}), 1.90(\mathrm{~s}, 3 \mathrm{H}) .{ }^{13} \mathrm{C} \mathrm{NMR}\left(\mathrm{CDCl}_{3}, 100 \mathrm{MHz}\right)$ : $\delta 168.9,168.6,139.9,134.72133 .4,128.8,128.7,128.6,52.7,52.6,16.6$. HRMS-ESI calcd for $[\mathrm{M}+\mathrm{Na}]^{+}: 257.0784$, found: 257.0789 . 


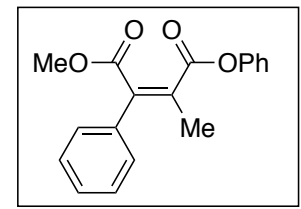

1-Methyl 4-phenyl 3-methyl-2-phenylmaleate

Yellow oil, $30 \%$ yield. ${ }^{1} \mathrm{H}$ NMR $\left(\mathrm{CDCl}_{3}, 400 \mathrm{MHz}\right): \delta 7.47-7.39(\mathrm{~m}, 5 \mathrm{H}), 7.38-7.33$ $(\mathrm{m}, 2 \mathrm{H}), 7.31-7.19(\mathrm{~m}, 3 \mathrm{H}), 3.75(\mathrm{~s}, 3 \mathrm{H}), 2.06(\mathrm{~s}, 3 \mathrm{H}) .{ }^{13} \mathrm{C} \mathrm{NMR}\left(\mathrm{CDCl}_{3}, 100 \mathrm{MHz}\right)$ : $\delta 168.3,167.0,150.8,140.5,134.6,133.5,129.7,128.8(2 \mathrm{C}), 128.7,128.7,126.2,121.6$, 52.8, 16.9. HRMS-ESI calcd for $[\mathrm{M}+\mathrm{Na}]^{+}: 319.0941$, found: 319.0951 .

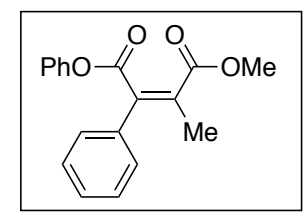

\section{1-Methyl 4-phenyl 2-methyl-3-phenylmaleate}

Yellow oil, $15 \%$ yield. ${ }^{1} \mathrm{H}$ NMR $\left(\mathrm{CDCl}_{3}, 400 \mathrm{MHz}\right): \delta 7.47-7.34(\mathrm{~m}, 7 \mathrm{H}), 7.25-7.19$ $(\mathrm{m}, 1 \mathrm{H}), 7.18-7.14(\mathrm{~m}, 2 \mathrm{H}), 3.86(\mathrm{~s}, 3 \mathrm{H}), 1.99(\mathrm{~s}, 3 \mathrm{H}) .{ }^{13} \mathrm{C} \mathrm{NMR}\left(\mathrm{CDCl}_{3}, 100 \mathrm{MHz}\right)$ : $\delta 168.5,166.7,150.7,140.2,134.5,133.2,129.5,128.9,128.8,128.8,126.1,121.5,52.7$, 16.4. HRMS-ESI calcd for $[\mathrm{M}+\mathrm{Na}]^{+}: 319.0941$, found: 319.0950 .

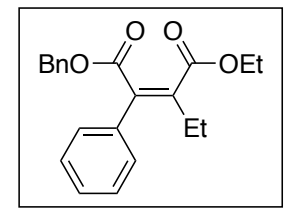

\section{1-Benzyl 4-ethyl 3-ethyl-2-phenylmaleate(1d)}

Colourless oil, $18 \%$ yield. ${ }^{1} \mathrm{H}$ NMR $\left(\mathrm{CDCl}_{3}, 400 \mathrm{MHz}\right): \delta 7.41-7.33(\mathrm{~m}, 3 \mathrm{H}), 7.33-$ $7.23(\mathrm{~m}, 7 \mathrm{H}), 5.17(\mathrm{~s}, 2 \mathrm{H}), 4.18(\mathrm{q}, J=7.1 \mathrm{~Hz}, 2 \mathrm{H}), 2.26(\mathrm{q}, J=7.5 \mathrm{~Hz}, 2 \mathrm{H}), 1.27$ (t, $J=$ $7.1 \mathrm{~Hz}, 3 \mathrm{H}), 1.02(\mathrm{t}, J=7.5 \mathrm{~Hz}, 3 \mathrm{H}) .{ }^{13} \mathrm{C} \mathrm{NMR}\left(\mathrm{CDCl}_{3}, 100 \mathrm{MHz}\right): \delta 168.4,167.6,141.6$, $137.3,135.7,135.0,128.8,128.6(2 \mathrm{C}), 128.4,128.3,128.2,67.1,61.4,24.2,14.2,13.0$. HRMS-ESI calcd for [M+Na] $]^{+}: 361.1410$, found: 361.1419 .

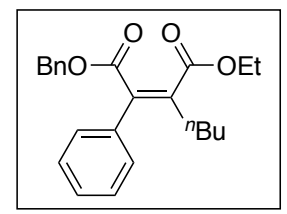

\section{1-Benzyl 4-ethyl 3-butyl-2-phenylmaleate (1e)}

Colourless oil, $22 \%$ yield. ${ }^{1} \mathrm{H}$ NMR $\left(\mathrm{CDCl}_{3}, 400 \mathrm{MHz}\right): \delta 7.41-7.22(\mathrm{~m}, 10 \mathrm{H}), 5.17(\mathrm{~s}$, $2 \mathrm{H}), 4.17(\mathrm{q}, J=7.1 \mathrm{~Hz}, 2 \mathrm{H}), 2.27-2.19(\mathrm{~m}, 2 \mathrm{H}), 1.44-1.35(\mathrm{~m}, 2 \mathrm{H}), 1.26(\mathrm{t}, J=7.1$ $\mathrm{Hz}, 3 \mathrm{H}), 1.24-1.16(\mathrm{~m}, 2 \mathrm{H}), 0.78(\mathrm{t}, J=7.3 \mathrm{~Hz}, 3 \mathrm{H}) .{ }^{13} \mathrm{C} \mathrm{NMR}\left(\mathrm{CDCl}_{3}, 100 \mathrm{MHz}\right)$ : $\delta 168.6,167.6,140.9,137.3,135.7,135.0,128.9,128.6,128.5,128.4,128.2,128.2,67.1$, $61.4,30.5$ (2C), 22.6, 14.2, 13.8. HRMS-ESI calcd for $[\mathrm{M}+\mathrm{Na}]^{+}: 389.1723$, found: 389.1726 . 


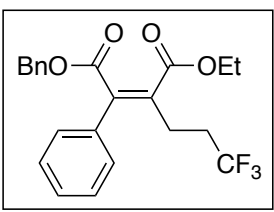

1-Benzyl 4-ethyl 2-phenyl-3-(3,3,3-trifluoropropyl)maleate (1f)

Colourless oil, $60 \%$ yield. ${ }^{1} \mathrm{H}$ NMR $\left(\mathrm{CDCl}_{3}, 400 \mathrm{MHz}\right): \delta 7.48-7.37(\mathrm{~m}, 3 \mathrm{H}), 7.37-$ $7.19(\mathrm{~m}, 7 \mathrm{H}), 5.20(\mathrm{~s}, 2 \mathrm{H}), 4.21(\mathrm{q}, J=7.2 \mathrm{~Hz}, 2 \mathrm{H}), 2.58-2.43(\mathrm{~m}, 2 \mathrm{H}), 2.33-2.14(\mathrm{~m}$, 2H), $1.29(\mathrm{t}, J=7.2 \mathrm{~Hz}, 3 \mathrm{H}) .{ }^{13} \mathrm{C} \mathrm{NMR}\left(\mathrm{CDCl}_{3}, 100 \mathrm{MHz}\right): \delta 167.6,166.7,142.8,135.4$, $134.1,133.3,129.1,128.9,128.6,128.4,128.3,128.1,67.4,61.8,32.8$ (q, $J=29.0 \mathrm{~Hz})$, $22.7(\mathrm{q}, J=3.4 \mathrm{~Hz}), 14.1{ }^{19} \mathrm{~F}$ NMR $\left(\mathrm{CDCl}_{3}, 376 \mathrm{MHz}\right) \delta-66.9$ (s). HRMS-ESI calcd for $[\mathrm{M}+\mathrm{Na}]^{+}:$429.1290, found: 429.1282 .

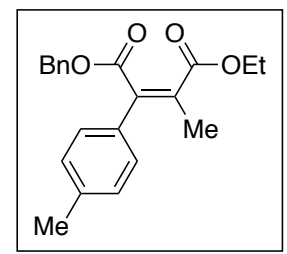

\section{1-Benzyl 4-ethyl 3-methyl-2-(p-tolyl)maleate (1g)}

Colourless oil, $46 \%$ yield. ${ }^{1} \mathrm{H}$ NMR $\left(\mathrm{CDCl}_{3}, 400 \mathrm{MHz}\right) \delta 7.36-7.27(\mathrm{~m}, 5 \mathrm{H}), 7.22$ - 7.16 $(\mathrm{m}, 4 \mathrm{H}), 5.20(\mathrm{~s}, 2 \mathrm{H}), 4.17(\mathrm{q}, J=7.1 \mathrm{~Hz}, 2 \mathrm{H}), 2.37(\mathrm{~s}, 3 \mathrm{H}), 1.92(\mathrm{~s}, 3 \mathrm{H}), 1.27(\mathrm{t}, J=7.1$ $\mathrm{Hz}, 3 \mathrm{H}) .{ }^{13} \mathrm{C} \mathrm{NMR}\left(\mathrm{CDCl}_{3}, 100 \mathrm{MHz}\right) \delta 168.3(2 \mathrm{C}), 140.0,138.6,135.7,132.7,131.9$, 129.3, 128.7, 128.6, 128.3, 128.3, 67.2, 61.5, 21.4, 16.5, 14.2. HRMS-ESI calcd for $[\mathrm{M}+\mathrm{Na}]^{+}:$361.1410, found: 361.1406 .

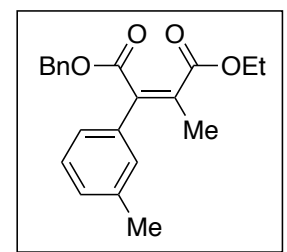

\section{1-Benzyl 4-ethyl 3-methyl-2-(m-tolyl)maleate(1h)}

Yellow oil, 23\% yield. ${ }^{1} \mathrm{H}$ NMR $\left(\mathrm{CDCl}_{3}, 400 \mathrm{MHz}\right): \delta 7.37-7.25(\mathrm{~m}, 7 \mathrm{H}), 7.17$ (dtd, $J=$ 7.6, 1.4, $0.8 \mathrm{~Hz}, 1 \mathrm{H}), 7.14-7.08(\mathrm{~m}, 2 \mathrm{H}), 5.21(\mathrm{~s}, 2 \mathrm{H}), 4.18(\mathrm{q}, J=7.1 \mathrm{~Hz}, 2 \mathrm{H}), 2.35$ (d, $J=0.7 \mathrm{~Hz}, 3 \mathrm{H}), 1.92(\mathrm{~s}, 3 \mathrm{H}), 1.28(\mathrm{t}, J=7.1 \mathrm{~Hz}, 3 \mathrm{H}) .{ }^{13} \mathrm{C} \mathrm{NMR}\left(\mathrm{CDCl}_{3}, 100 \mathrm{MHz}\right)$ : $\delta 168.3,140.0,138.3,135.8,134.8,133.0,129.4,129.3,128.6,128.5,128.3,128.3$, $125.9,67.2,61.5,21.5,16.6,14.2$. HRMS-ESI calcd for $[\mathrm{M}+\mathrm{Na}]^{+}: 361.1410$, found: 361.1426 .

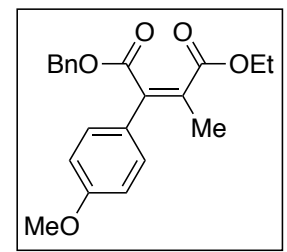


1-Benzyl 4-ethyl 2-(4-methoxyphenyl)-3-methylmaleate (1i)

Colourless oil, $48 \%$ yield. ${ }^{1} \mathrm{H}$ NMR $\left(\mathrm{CDCl}_{3}, 400 \mathrm{MHz}\right): \delta 7.35-7.28(\mathrm{~m}, 5 \mathrm{H}), 7.25-$ $7.20(\mathrm{~m}, 2 \mathrm{H}), 6.93-6.87(\mathrm{~m}, 2 \mathrm{H}), 5.20(\mathrm{~s}, 2 \mathrm{H}), 4.17(\mathrm{q}, J=7.1 \mathrm{~Hz}, 2 \mathrm{H}), 3.82(\mathrm{~s}, 3 \mathrm{H})$, $1.93(\mathrm{~s}, 3 \mathrm{H}), 1.27(\mathrm{t}, J=7.1 \mathrm{~Hz}, 3 \mathrm{H}) .{ }^{13} \mathrm{C} \mathrm{NMR}\left(\mathrm{CDCl}_{3}, 100 \mathrm{MHz}\right): \delta 168.5,168.3$, $159.9,139.9,135.7,132.1,130.3,128.6,128.4,128.3,127.0,114.0,67.2,61.5,55.4$, 16.5, 14.2. HRMS-ESI calcd for $[\mathrm{M}+\mathrm{Na}]^{+}: 377.1359$, found: 377.1363 .

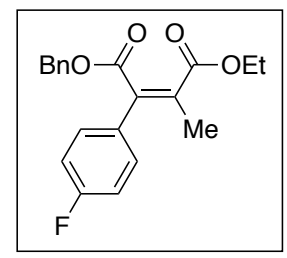

\section{1-Benzyl 4-ethyl 2-(4-fluorophenyl)-3-methylmaleate (1j)}

Colourless oil, 53\% yield. ${ }^{1} \mathrm{H}$ NMR $\left(\mathrm{CDCl}_{3}, 400 \mathrm{MHz}\right): \delta 7.36-7.23(\mathrm{~m}, 7 \mathrm{H}), 7.10-$ 7.03 (m, 2H), 5.19 (s, 2H), 4.17 (q, $J=7.1 \mathrm{~Hz}, 2 \mathrm{H}), 1.90$ (s, 3H), 1.27 (t, $J=7.1 \mathrm{~Hz}, 3 \mathrm{H})$.

${ }^{13} \mathrm{C} \mathrm{NMR}\left(\mathrm{CDCl}_{3}, 100 \mathrm{MHz}\right): \delta 168.2,167.7,162.8(\mathrm{~d}, J=248.5 \mathrm{~Hz}), 138.2,135.5$, $134.4,130.8(\mathrm{~d}, J=8.4 \mathrm{~Hz}), 128.6,128.4(\mathrm{~d}, J=5.8 \mathrm{~Hz}), 115.8,115.6,67.4,61.6,16.8$, 14.2. ${ }^{19} \mathrm{~F}$ NMR $\left(\mathrm{CDCl}_{3}, 376 \mathrm{MHz}\right) \delta-112.7$ (s). HRMS-ESI calcd for $[\mathrm{M}+\mathrm{Na}]^{+}$: 365.1160, found: 365.1169 .

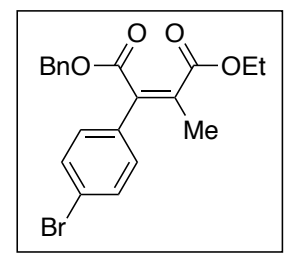

\section{1-Benzyl 4-ethyl 2-(4-bromophenyl)-3-methylmaleate (1k)}

Colourless oil, 43\% yield. ${ }^{1} \mathrm{H}$ NMR $\left(\mathrm{CDCl}_{3}, 400 \mathrm{MHz}\right) \delta 7.55-7.48(\mathrm{~m}, 2 \mathrm{H}), 7.36$ - 7.26 $(\mathrm{m}, 5 \mathrm{H}), 7.20-7.11(\mathrm{~m}, 2 \mathrm{H}), 5.18(\mathrm{~s}, 2 \mathrm{H}), 4.17(\mathrm{q}, J=7.1 \mathrm{~Hz}, 2 \mathrm{H}), 1.89(\mathrm{~s}, 3 \mathrm{H}), 1.27(\mathrm{t}$, $J=7.1 \mathrm{~Hz}, 3 \mathrm{H}) .{ }^{13} \mathrm{C} \mathrm{NMR}\left(\mathrm{CDCl}_{3}, 100 \mathrm{MHz}\right) \delta 168.2,167.4,137.8,135.5,135.0,131.8$, 130.6, 128.6, 128.4, 128.4, 122.9, 67.4, 61.7, 16.8, 14.1. HRMS-ESI calcd for $[\mathrm{M}+\mathrm{Na}]^{+}$, $[\mathrm{M}+\mathrm{Na}+2]^{+}:$425.0359, found: 425.0348, 427.0335 .

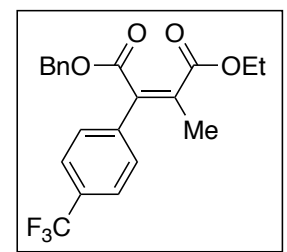

1-Benzyl 4-ethyl 3-methyl-2-(4-(trifluoromethyl)phenyl)maleate (11)

Colourless oil, $53 \%$ yield ${ }^{1} \mathrm{H}$ NMR $\left(\mathrm{CDCl}_{3}, 400 \mathrm{MHz}\right) \delta 7.68-7.62(\mathrm{~m}, 2 \mathrm{H}), 7.44-7.38$ $(\mathrm{m}, 2 \mathrm{H}), 7.36-7.30(\mathrm{~m}, 3 \mathrm{H}), 7.29-7.25(\mathrm{~m}, 2 \mathrm{H}), 5.19(\mathrm{~s}, 2 \mathrm{H}), 4.18(\mathrm{q}, J=7.1 \mathrm{~Hz}, 2 \mathrm{H})$, $1.89(\mathrm{~s}, 3 \mathrm{H}), 1.27(\mathrm{t}, J=7.2 \mathrm{~Hz}, 3 \mathrm{H}) .{ }^{13} \mathrm{C} \mathrm{NMR}\left(\mathrm{CDCl}_{3}, 100 \mathrm{MHz}\right) \delta 168.0,166.8,138.4$, $136.9,136.2,135.2,130.5(\mathrm{q}, I J C-F=32.6 \mathrm{~Hz}), 129.3,128.5,128.3,128.2,125.46(\mathrm{q}$, 
$2 J C-F=3.8 \mathrm{~Hz}), 122.6,67.4,61.6,16.9,14.0 .{ }^{19} \mathrm{~F}$ NMR $\left(\mathrm{CDCl}_{3}, 376 \mathrm{MHz}\right) \delta-62.7(\mathrm{~s})$. HRMS-ESI calcd for $[\mathrm{M}+\mathrm{Na}]^{+}: 415.1128$, found: 415.1128 .

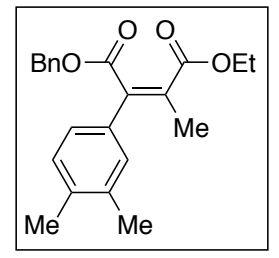

1-Benzyl 4-ethyl 2-(3,4-dimethylphenyl)-3-methylmaleate (1m)

Yellow oil, $43 \%$ yield. ${ }^{1} \mathrm{H}$ NMR $\left(\mathrm{CDCl}_{3}, 400 \mathrm{MHz}\right): \delta 7.36-7.28(\mathrm{~m}, 5 \mathrm{H}), 7.17-7.10$ $(\mathrm{m}, 1 \mathrm{H}), 7.08-7.00(\mathrm{~m}, 2 \mathrm{H}), 5.20(\mathrm{~s}, 2 \mathrm{H}), 4.16(\mathrm{q}, J=7.1 \mathrm{~Hz}, 2 \mathrm{H}), 2.27(\mathrm{~s}, 3 \mathrm{H}), 2.24(\mathrm{~s}$, $3 \mathrm{H}), 1.92(\mathrm{~s}, 3 \mathrm{H}), 1.26(\mathrm{t}, J=7.1 \mathrm{~Hz}, 3 \mathrm{H}) .{ }^{13} \mathrm{C}$ NMR $\left(\mathrm{CDCl}_{3}, 100 \mathrm{MHz}\right): \delta 168.5,168.3$, $140.3,137.3,136.8,135.8,132.3,132.2,129.8,128.6,128.4,128.3,126.3,67.2,61.4$, $19.9,19.8,16.5,14.2$. HRMS-ESI calcd for $[\mathrm{M}+\mathrm{Na}]^{+}: 375.1567$, found: 375.1568 .

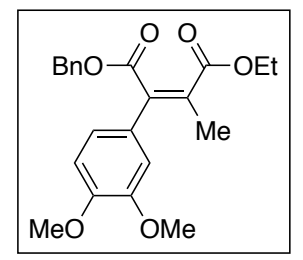

1-Benzyl 4-ethyl 2-(3,4-dimethoxyphenyl)-3-methylmaleate (1n)

Colourless oil, $50 \%$ yield. ${ }^{1} \mathrm{H}$ NMR (CDCl $\left.3,400 \mathrm{MHz}\right): \delta 7.35-7.28(\mathrm{~m}, 5 \mathrm{H}), 6.86(\mathrm{~d}, J$ $=1.1 \mathrm{~Hz}, 2 \mathrm{H}), 6.78(\mathrm{~d}, J=1.2 \mathrm{~Hz}, 1 \mathrm{H}), 5.21(\mathrm{~s}, 2 \mathrm{H}), 4.18(\mathrm{q}, J=7.1 \mathrm{~Hz}, 2 \mathrm{H}), 3.89(\mathrm{~s}$, $3 \mathrm{H}), 3.77(\mathrm{~s}, 3 \mathrm{H}), 1.94(\mathrm{~s}, 3 \mathrm{H}), 1.28(\mathrm{t}, J=7.1 \mathrm{~Hz}, 3 \mathrm{H}) .{ }^{13} \mathrm{C} \mathrm{NMR}\left(\mathrm{CDCl}_{3}, 100 \mathrm{MHz}\right)$ : $\delta 168.3,168.2,149.3,148.9,139.9,135.7,132.4,128.6,128.5,128.4,127.2,121.7$, $111.8,111.0,67.2,61.5,56.0,56.0,16.6,14.2$. HRMS-ESI calcd for $[\mathrm{M}+\mathrm{Na}]^{+}: 407.1471$, found: 407.1455 .

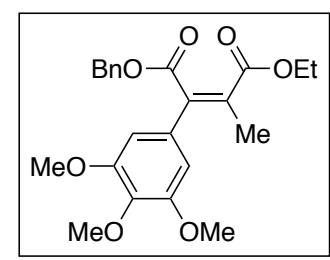

\section{1-Benzyl 4-ethyl 3-methyl-2-(3,4,5-trimethoxyphenyl)maleate (10)}

Colourless oil, $56 \%$ yield. ${ }^{1} \mathrm{H}$ NMR $\left(\mathrm{CDCl}_{3}, 400 \mathrm{MHz}\right): \delta 7.32(\mathrm{~d}, J=1.0 \mathrm{~Hz}, 5 \mathrm{H}), 6.48$ $(\mathrm{d}, J=0.6 \mathrm{~Hz}, 2 \mathrm{H}), 5.22(\mathrm{~d}, J=0.7 \mathrm{~Hz}, 2 \mathrm{H}), 4.19(\mathrm{qd}, J=7.2,0.8 \mathrm{~Hz}, 2 \mathrm{H}), 3.86(\mathrm{~d}, J=$ $0.9 \mathrm{~Hz}, 3 \mathrm{H}), 3.77(\mathrm{~d}, J=0.9 \mathrm{~Hz}, 6 \mathrm{H}), 1.94(\mathrm{~d}, J=0.6 \mathrm{~Hz}, 3 \mathrm{H}), 1.28$ (td, $J=7.1,0.9 \mathrm{~Hz}$, $3 \mathrm{H}) .{ }^{13} \mathrm{C}$ NMR $\left(\mathrm{CDCl}_{3}, 100 \mathrm{MHz}\right): \delta 168.1,168.0,153.3,139.8,138.2,135.7,133.1$, $130.1,128.6,128.5,128.4,105.9,67.3,61.6,61.0,56.2,16.7,14.2$. HRMS-ESI calcd for $[\mathrm{M}+\mathrm{Na}]^{+}:$437.1576, found: 437.1584 . 


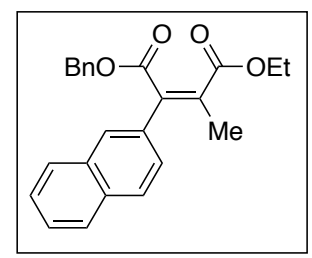

\section{1-Benzyl 4-ethyl 3-methyl-2-(naphthalen-2-yl)maleate (1p)}

Yellow oil, 52\% yield. ${ }^{1} \mathrm{H}$ NMR $\left(\mathrm{CDCl}_{3}, 400 \mathrm{MHz}\right): \delta 7.89-7.75(\mathrm{~m}, 4 \mathrm{H}), 7.56-7.48$ $(\mathrm{m}, 2 \mathrm{H}), 7.39(\mathrm{dd}, J=8.5,1.8 \mathrm{~Hz}, 1 \mathrm{H}), 7.29(\mathrm{~s}, 5 \mathrm{H}), 5.22(\mathrm{~s}, 2 \mathrm{H}), 4.20(\mathrm{q}, J=7.1 \mathrm{~Hz}$, $2 \mathrm{H}), 1.96(\mathrm{~s}, 3 \mathrm{H}), 1.29(\mathrm{t}, J=7.2 \mathrm{~Hz}, 3 \mathrm{H}) .{ }^{13} \mathrm{C} \mathrm{NMR}\left(\mathrm{CDCl}_{3}, 100 \mathrm{MHz}\right): \delta 168.3,168.0$, 139.6, 135.7, 133.9, 133.2, 132.3, 128.6, 128.4 (2C), 128.3(2C), 127.9, 126.8, 126.6, 126.4, 67.3, 61.6, 16.8, 14.2. HRMS-ESI calcd for [M+Na] ${ }^{+}: 397.1410$, found: 397.1413.

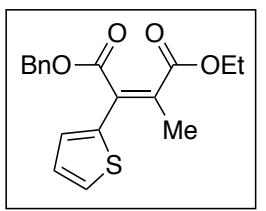

\section{1-Benzyl 4-ethyl 3-methyl-2-(thiophen-2-yl)maleate (1q)}

Yellow oil, 58\% yield. ${ }^{1} \mathrm{H}$ NMR $\left(\mathrm{CDCl}_{3}, 400 \mathrm{MHz}\right): \delta 7.45(\mathrm{dd}, J=5.1,1.2 \mathrm{~Hz}, 1 \mathrm{H})$, $7.40-7.30(\mathrm{~m}, 5 \mathrm{H}), 7.09(\mathrm{dd}, J=3.8,1.2 \mathrm{~Hz}, 1 \mathrm{H}), 7.05(\mathrm{dd}, J=5.1,3.7 \mathrm{~Hz}, 1 \mathrm{H}), 5.28$ $(\mathrm{s}, 2 \mathrm{H}), 4.18(\mathrm{q}, J=7.2 \mathrm{~Hz}, 2 \mathrm{H}), 2.17(\mathrm{~s}, 3 \mathrm{H}), 1.28(\mathrm{t}, J=7.2 \mathrm{~Hz}, 3 \mathrm{H}) .{ }^{13} \mathrm{C} \mathrm{NMR}\left(\mathrm{CDCl}_{3}\right.$, $100 \mathrm{MHz}$ ): $\delta 168.0,167.7,135.9,135.5,134.8,130.5,130.0,128.7,128.6$ (2C), 128.5, 127.2, 67.7, 61.7, 16.7, 14.2. HRMS-ESI calcd for $[\mathrm{M}+\mathrm{Na}]^{+}: 353.0818$, found: 353.0832 .

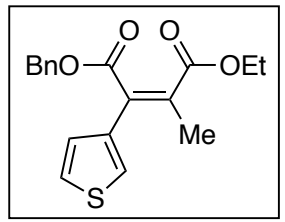

\section{1-Benzyl 4-ethyl 3-methyl-2-(thiophen-3-yl)maleate (1r)}

Colourless oil, 48\% yield. ${ }^{1} \mathrm{H}$ NMR $\left(\mathrm{CDCl}_{3}, 400 \mathrm{MHz}\right): \delta 7.36-7.29(\mathrm{~m}, 7 \mathrm{H}), 7.07$ (dd, $J=4.5,1.8 \mathrm{~Hz}, 1 \mathrm{H}), 5.23(\mathrm{~s}, 2 \mathrm{H}), 4.17(\mathrm{q}, J=7.1 \mathrm{~Hz}, 2 \mathrm{H}), 2.03(\mathrm{~s}, 3 \mathrm{H}), 1.27$ (t, $J=7.1$ $\mathrm{Hz}, 3 \mathrm{H}) .{ }^{13} \mathrm{C} \mathrm{NMR}\left(\mathrm{CDCl}_{3}, 100 \mathrm{MHz}\right): \delta 168.1,168.0,135.6,135.5,134.7,132.1,128.6$, $128.5,128.4,128.2,126.0,125.8,67.4,61.6,16.7,14.2$. HRMS-ESI calcd for $[\mathrm{M}+\mathrm{Na}]^{+}$: 353.0818, found: 353.0818 .

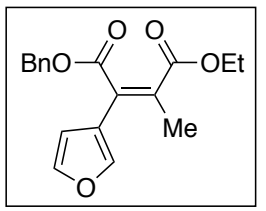

\section{1-Benzyl 4-ethyl 2-(furan-3-yl)-3-methylmaleate (1s)}

Colourless oil, $36 \%$ yield. ${ }^{1} \mathrm{H}$ NMR $\left(\mathrm{CDCl}_{3}, 400 \mathrm{MHz}\right): \delta 7.51(\mathrm{dd}, J=1.5,0.9 \mathrm{~Hz}, 1 \mathrm{H})$, $7.44-7.41(\mathrm{~m}, 1 \mathrm{H}), 7.40-7.29(\mathrm{~m}, 5 \mathrm{H}), 6.44(\mathrm{dd}, J=1.9,0.9 \mathrm{~Hz}, 1 \mathrm{H}), 5.25(\mathrm{~s}, 2 \mathrm{H})$, 
4.17 (q, $J=7.1 \mathrm{~Hz}, 2 \mathrm{H}), 2.08$ (s, 3H), 1.27 (t, $J=7.1 \mathrm{~Hz}, 3 \mathrm{H}) .{ }^{13} \mathrm{C}$ NMR $\left(\mathrm{CDCl}_{3}, 100\right.$ MHz): $\delta 168.2,167.8,143.2,143.0,135.5,132.4,130.8,128.7,128.6,128.5,119.8$, $110.9,67.5,61.5,16.6,14.2$. HRMS-ESI calcd for $[\mathrm{M}+\mathrm{Na}]^{+}: 337.1046$, found: 337.1058 .

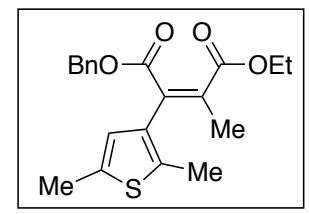

\section{1-Benzyl 4-ethyl 2-(2,5-dimethylthiophen-3-yl)-3-methylmaleate (1t)}

Yellow oil, $49 \%$ yield. ${ }^{1} \mathrm{H}$ NMR $\left(\mathrm{CDCl}_{3}, 400 \mathrm{MHz}\right): \delta 7.36-7.27(\mathrm{~m}, 5 \mathrm{H}), 6.51-6.44$ (m, 1H), 5.18 (s, 2H), 4.15 (q, J = 7.1 Hz, 2H), 2.38 (s, 3H), 2.23 (s, 3H), $1.84(\mathrm{~s}, 3 \mathrm{H})$, $1.25(\mathrm{t}, J=7.1 \mathrm{~Hz}, 3 \mathrm{H}) .{ }^{13} \mathrm{C} \mathrm{NMR}\left(\mathrm{CDCl}_{3}, 100 \mathrm{MHz}\right): \delta 168.1,167.7,136.6,135.8$, 135.4, 135.0, 134.8, 130.9, 128.6, 128.3, 128.2, 125.9, 67.0, 61.5, 16.7, 15.3, 14.2, 14.0. HRMS-ESI calcd for $[\mathrm{M}+\mathrm{Na}]^{+}: 381.1131$, found: 381.1120 .

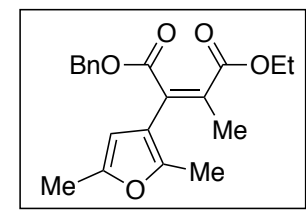

\section{1-Benzyl 4-ethyl 2-(2,5-dimethylfuran-3-yl)-3-methylmaleate (1u)}

Yellow oil, $45 \%$ yield. ${ }^{1} \mathrm{H}$ NMR $\left(\mathrm{CDCl}_{3}, 400 \mathrm{MHz}\right): \delta 7.38-7.28(\mathrm{~m}, 5 \mathrm{H}), 5.86-5.84$ (m, 1H), $5.20(\mathrm{~s}, 2 \mathrm{H}), 4.14(\mathrm{q}, J=7.1 \mathrm{~Hz}, 2 \mathrm{H}), 2.22(\mathrm{~s}, 3 \mathrm{H}), 2.11(\mathrm{~s}, 3 \mathrm{H}), 1.91(\mathrm{~s}, 3 \mathrm{H})$, $1.25(\mathrm{t}, J=7.1 \mathrm{~Hz}, 3 \mathrm{H}) .{ }^{13} \mathrm{C} \mathrm{NMR}\left(\mathrm{CDCl}_{3}, 100 \mathrm{MHz}\right): \delta 168.2,168.1,150.4,148.5$, $135.8,133.2,132.9,128.6,128.5,128.3,115.3,107.5,67.2,61.4,16.9,14.2,13.5,13.0$. HRMS-ESI calcd for $[\mathrm{M}+\mathrm{Na}]^{+}: 365.1359$, found: 365.1312 .

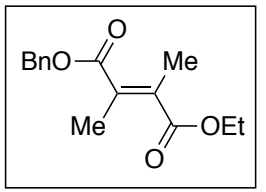

\section{1-Benzyl 4-ethyl 2,3-dimethylfumarate (1v)}

Colourless oil, $58 \%$ yield. ${ }^{1} \mathrm{H}$ NMR $\left(\mathrm{CDCl}_{3}, 400 \mathrm{MHz}\right): \delta 7.39-7.32(\mathrm{~m}, 5 \mathrm{H}), 5.22(\mathrm{~s}$, $2 \mathrm{H}), 4.24(\mathrm{q}, J=7.1 \mathrm{~Hz}, 2 \mathrm{H}), 2.07-2.03(\mathrm{~m}, 6 \mathrm{H}), 1.31(\mathrm{t}, J=7.1 \mathrm{~Hz}, 3 \mathrm{H}) .{ }^{13} \mathrm{C}$ NMR $\left(\mathrm{CDCl}_{3}, 100 \mathrm{MHz}\right): \delta 169.2,168.8,135.8,134.6,132.7,128.8,128.5,128.4,66.8,61.09$, 17.6, 17.5, 14.3. HRMS-ESI calcd for $[\mathrm{M}+\mathrm{Na}]^{+}: 285.1097$, found: 285.1109 .

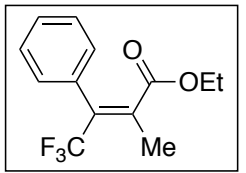

Ethyl (E)-4,4,4-trifluoro-2-methyl-3-phenylbut-2-enoate (5a)

Colourless oil, 89\% yield. ${ }^{1} \mathrm{H}$ NMR $\left(\mathrm{CDCl}_{3}, 400 \mathrm{MHz}\right) \delta 7.36-7.31(\mathrm{~m}, 3 \mathrm{H}), 7.23-7.20$ $(\mathrm{m}, 2 \mathrm{H}), 3.87(\mathrm{q}, J=7.1 \mathrm{~Hz}, 2 \mathrm{H}), 2.25(\mathrm{q}, J=2.66 \mathrm{~Hz}, 3 \mathrm{H}), 0.82(\mathrm{t}, J=7.14 \mathrm{~Hz}, 3 \mathrm{H}) .{ }^{13} \mathrm{C}$ 
NMR $\left(\mathrm{CDCl}_{3}, 100 \mathrm{MHz}\right) \delta 168.9,139.3(\mathrm{q}, J=2.97 \mathrm{~Hz}), 134.0(\mathrm{q}, J=1.72 \mathrm{~Hz}), 132.5$ (d, $J=30.99 \mathrm{~Hz}), 129.4,128.7,128.2,123.5(\mathrm{~d}, J=276.01 \mathrm{~Hz}), 61.2,16.7(\mathrm{~d}, J=2.37$ $\mathrm{Hz})$, 13.5. ${ }^{19} \mathrm{~F}$ NMR $\left(\mathrm{CDCl}_{3}, 376 \mathrm{MHz}\right) \delta-58.3(\mathrm{~s})$. HRMS-ESI calcd for $[\mathrm{M}+\mathrm{Na}]^{+}$: 281.0782, found: 281.0760 .

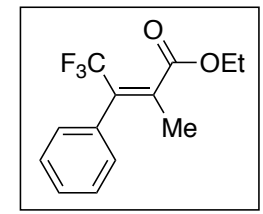

\section{Ethyl (Z)-4,4,4-trifluoro-2-methyl-3-phenylbut-2-enoate (5b)}

Colourless oil, $73 \%$ yield. ${ }^{1} \mathrm{H}$ NMR $\left(\mathrm{CDCl}_{3}, 400 \mathrm{MHz}\right) \delta 7.46-7.38(\mathrm{~m}, 3 \mathrm{H}), 7.28-7.24$ $(\mathrm{m}, 2 \mathrm{H}), 4.32(\mathrm{q}, J=7.1 \mathrm{~Hz}, 2 \mathrm{H}), 1.82(\mathrm{q}, J=2.2 \mathrm{~Hz}, 3 \mathrm{H}), 1.36(\mathrm{t}, J=7.1 \mathrm{~Hz}, 3 \mathrm{H}) .{ }^{13} \mathrm{C}$ NMR $\left(\mathrm{CDCl}_{3}, 100 \mathrm{MHz}\right) \delta 169.2,137.3(\mathrm{~d}, J=3.7 \mathrm{~Hz}), 132.2,129.6,129.0,128.8$, 124.0, 121.3, 61.9, 18.5, 14.1. ${ }^{19} \mathrm{~F}$ NMR $\left(\mathrm{CDCl}_{3}, 376 \mathrm{MHz}\right) \delta-61.4$ (s). HRMS-ESI calcd for $[\mathrm{M}+\mathrm{Na}]^{+}: 281.0782$, found: 281.0760 .

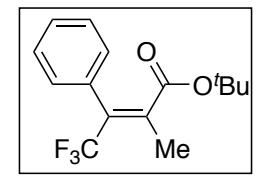

(E)-tert-butyl 4,4,4-trifluoro-2-methyl-3-phenylbut-2-enoate (5c)

Colourless oil, $81 \%$ yield. ${ }^{1} \mathrm{H}$ NMR $\left(\mathrm{CDCl}_{3}, 400 \mathrm{MHz}\right) \delta 7.38-7.29(\mathrm{~m}, 3 \mathrm{H}), 7.22(\mathrm{dd}, J$ $=6.6,3.0 \mathrm{~Hz}, 2 \mathrm{H}), 2.20(\mathrm{q}, J=2.7 \mathrm{~Hz}, 3 \mathrm{H}), 1.10(\mathrm{~s}, 9 \mathrm{H}) \cdot{ }^{13} \mathrm{C} \mathrm{NMR}\left(\mathrm{CDCl}_{3}, 100 \mathrm{MHz}\right) \delta$ $168.1,140.3(\mathrm{~d}, J=3.0 \mathrm{~Hz}), 134.1$ (d, $J=1.7 \mathrm{~Hz}), 131.1$ (q, $J=30.8 \mathrm{~Hz}), 129.8,128.6$, 128.2, $123.7(\mathrm{~d}, J=275.8 \mathrm{~Hz}), 82.4,27.5,16.6(\mathrm{~d}, J=2.3 \mathrm{~Hz}) .{ }^{19} \mathrm{~F} \mathrm{NMR}\left(\mathrm{CDCl}_{3}, 376\right.$ $\mathrm{MHz}) \delta-58.3(\mathrm{~s})$. HRMS-ESI calcd for $[\mathrm{M}+\mathrm{Na}]^{+}: 309.1078$, found: 309.1081.

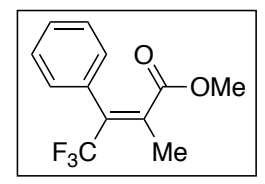

(E)-methyl 4,4,4-trifluoro-2-methyl-3-phenylbut-2-enoate (5d)

Colourless oil, 86\% yield. ${ }^{1} \mathrm{H}$ NMR $\left(\mathrm{CDCl}_{3}, 400 \mathrm{MHz}\right) \delta 7.40-7.30(\mathrm{~m}, 3 \mathrm{H}), 7.24-7.17$ $(\mathrm{m}, 2 \mathrm{H}), 3.40(\mathrm{~s}, 3 \mathrm{H}), 2.24(\mathrm{q}, J=2.7 \mathrm{~Hz}, 3 \mathrm{H}) \cdot{ }^{13} \mathrm{C} \mathrm{NMR}\left(\mathrm{CDCl}_{3}, 100 \mathrm{MHz}\right) \delta 169.3$, $139.1(\mathrm{~d}, J=3.0 \mathrm{~Hz}), 133.9(\mathrm{q}, J=1.6 \mathrm{~Hz}), 133.3-132.3(\mathrm{~m}), 129.1,128.8,128.2,123.5$ $(\mathrm{d}, J=276.0 \mathrm{~Hz}), 52.00,16.7(\mathrm{~d}, J=2.3 \mathrm{~Hz}) .{ }^{19} \mathrm{~F} \mathrm{NMR}\left(\mathrm{CDCl}_{3}, 376 \mathrm{MHz}\right) \delta-58.2(\mathrm{~s})$. HRMS-ESI calcd for $[\mathrm{M}+\mathrm{Na}]^{+}: 267.0609$, found: 267.0618 .

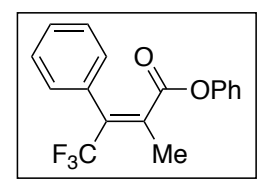

(E)-phenyl 4,4,4-trifluoro-2-methyl-3-phenylbut-2-enoate (5e)

Colourless oil, 79\% yield. ${ }^{1} \mathrm{H}$ NMR $\left(\mathrm{CDCl}_{3}, 400 \mathrm{MHz}\right) \delta 7.49-7.44(\mathrm{~m}, 3 \mathrm{H}), 7.41$ (dq, $J$ $=4.4,2.8 \mathrm{~Hz}, 2 \mathrm{H}), 7.32-7.26(\mathrm{~m}, 2 \mathrm{H}), 7.23-7.17(\mathrm{~m}, 1 \mathrm{H}), 6.55(\mathrm{ddd}, J=8.4,2.4,1.1$ $\mathrm{Hz}, 2 \mathrm{H}), 2.45(\mathrm{q}, J=2.7 \mathrm{~Hz}, 3 \mathrm{H}) .{ }^{13} \mathrm{C} \mathrm{NMR}\left(\mathrm{CDCl}_{3}, 100 \mathrm{MHz}\right) \delta 167.5,149.9,138.6(\mathrm{t}$, 
$J=2.9 \mathrm{~Hz}), 133.8(\mathrm{~d}, J=1.6 \mathrm{~Hz}), 133.7-132.8(\mathrm{~m}), 129.6,129.4,129.1,128.5,126.3$, $121.9(\mathrm{~d}, J=18.8 \mathrm{~Hz}), 121.1,16.8(\mathrm{t}, J=2.4 \mathrm{~Hz}) .{ }^{19} \mathrm{~F} \mathrm{NMR}\left(\mathrm{CDCl}_{3}, 376 \mathrm{MHz}\right) \delta-58.4$ (s). HRMS-ESI calcd for $[\mathrm{M}+\mathrm{Na}]^{+}: 329.0765$, found: 329.0746 .

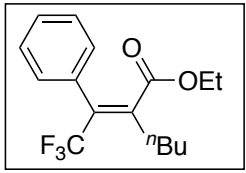

(E)-ethyl 2-(2,2,2-trifluoro-1-phenylethylidene)hexanoate (5f)

Colourless oil, $62 \%$ yield. ${ }^{1} \mathrm{H}$ NMR $\left(\mathrm{CDCl}_{3}, 400 \mathrm{MHz}\right) \delta 7.37-7.28(\mathrm{~m}, 3 \mathrm{H}), 7.24-7.17$ $(\mathrm{m}, 2 \mathrm{H}), 3.85(\mathrm{q}, J=7.1 \mathrm{~Hz}, 2 \mathrm{H}), 2.63(\mathrm{ddq}, J=10.1,6.1,2.0 \mathrm{~Hz}, 2 \mathrm{H}), 1.53-1.32(\mathrm{~m}$, $4 \mathrm{H}), 0.94(\mathrm{t}, J=7.2 \mathrm{~Hz}, 3 \mathrm{H}), 0.81(\mathrm{t}, J=7.1 \mathrm{~Hz}, 3 \mathrm{H}) \cdot{ }^{13} \mathrm{C} \mathrm{NMR}\left(\mathrm{CDCl}_{3}, 100 \mathrm{MHz}\right) \delta$ $168.6,145.6-143.7(\mathrm{~m}), 134.1(\mathrm{q}, J=1.8 \mathrm{~Hz}), 131.4(\mathrm{q}, J=30.9 \mathrm{~Hz}), 129.5,128.7$, $128.2,123.6(\mathrm{~d}, J=276.1 \mathrm{~Hz}), 61.1,30.6(\mathrm{~d}, J=2.2 \mathrm{~Hz}), 30.4,22.6,13.9,13.6 .{ }^{19} \mathrm{~F} \mathrm{NMR}$ $\left(\mathrm{CDCl}_{3}, 376 \mathrm{MHz}\right) \delta-57.4(\mathrm{~s})$. HRMS-ESI calcd for $[\mathrm{M}+\mathrm{Na}]^{+}$: 323.1235, found: 323.1232 .

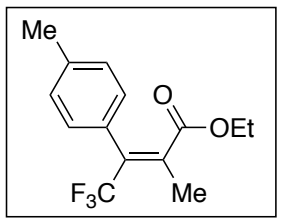

(E)-ethyl 4,4,4-trifluoro-2-methyl-3-p-tolylbut-2-enoate (5g)

Colourless oil, 48\% yield. ${ }^{1} \mathrm{H}$ NMR $\left(\mathrm{CDCl}_{3}, 400 \mathrm{MHz}\right) \delta 7.19-7.07(\mathrm{~m}, 4 \mathrm{H}), 3.90(\mathrm{q}, J$ $=7.1 \mathrm{~Hz}, 2 \mathrm{H}), 2.35(\mathrm{~d}, J=0.6 \mathrm{~Hz}, 3 \mathrm{H}), 2.23(\mathrm{q}, J=2.6 \mathrm{~Hz}, 3 \mathrm{H}), 0.87(\mathrm{t}, J=7.1 \mathrm{~Hz}, 3 \mathrm{H})$. ${ }^{13} \mathrm{C}$ NMR $\left(\mathrm{CDCl}_{3}, 100 \mathrm{MHz}\right) \delta 169.0,139.1(\mathrm{q}, J=3.0 \mathrm{~Hz}), 138.6,132.4(\mathrm{q}, J=30.9$ $\mathrm{Hz}), 131.0(\mathrm{~d}, J=1.6 \mathrm{~Hz}), 129.2,128.9,123.6(\mathrm{~d}, J=275.9 \mathrm{~Hz}), 61.2,21.3,16.6(\mathrm{~d}, J=$ $2.5 \mathrm{~Hz}), 13.5 .{ }^{19} \mathrm{~F} \mathrm{NMR}\left(\mathrm{CDCl}_{3}, 376 \mathrm{MHz}\right) \delta-58.4$ (s). HRMS-ESI calcd for $[\mathrm{M}+\mathrm{Na}]^{+}$: 295.0922, found: 295.0925 .

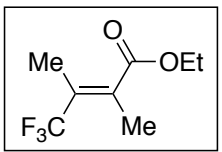

(E)-ethyl 4,4,4-trifluoro-2,3-dimethylbut-2-enoate (5h)

Colourless oil, $66 \%$ yield. ${ }^{1} \mathrm{H}$ NMR $\left(\mathrm{CDCl}_{3}, 400 \mathrm{MHz}\right) \delta 4.26(\mathrm{q}, J=7.1 \mathrm{~Hz}, 2 \mathrm{H}), 2.05$ $(\mathrm{qd}, J=2.7,1.4 \mathrm{~Hz}, 3 \mathrm{H}), 1.92(\mathrm{q}, J=1.6 \mathrm{~Hz}, 3 \mathrm{H}), 1.33(\mathrm{t}, J=7.1 \mathrm{~Hz}, 3 \mathrm{H}) .{ }^{13} \mathrm{C} \mathrm{NMR}$ $\left(\mathrm{CDCl}_{3}, 100 \mathrm{MHz}\right) \delta 169.1,135.2(\mathrm{t}, J=3.3 \mathrm{~Hz}), 128.5(\mathrm{q}, J=29.7 \mathrm{~Hz}), 124.2(\mathrm{~d}, J=$ $276.2 \mathrm{~Hz}), 61.5,16.4(\mathrm{q}, J=2.4 \mathrm{~Hz}), 15.3$ (q, $J=3.0 \mathrm{~Hz}), 14.2 .{ }^{19} \mathrm{~F} \mathrm{NMR}\left(\mathrm{CDCl}_{3}, 376\right.$ $\mathrm{MHz}) \delta-61.6(\mathrm{~s})$.

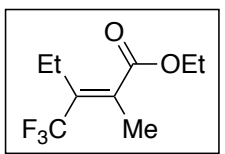

(E)-ethyl 2-methyl-3-(trifluoromethyl)pent-2-enoate(5i) 
Colourless oil, 91\% yield. ${ }^{1} \mathrm{H}$ NMR $\left(\mathrm{CDCl}_{3}, 400 \mathrm{MHz}\right) \delta 4.26(\mathrm{q}, J=7.2 \mathrm{~Hz}, 2 \mathrm{H}), 2.37-$ $2.20(\mathrm{~m}, 2 \mathrm{H}), 2.04(\mathrm{dtd}, J=3.5,2.6,0.9 \mathrm{~Hz}, 3 \mathrm{H}), 1.33(\mathrm{t}, J=7.1 \mathrm{~Hz}, 3 \mathrm{H}), 1.16-1.02(\mathrm{~m}$, $3 \mathrm{H}) .{ }^{13} \mathrm{C} \mathrm{NMR}\left(\mathrm{CDCl}_{3}, 100 \mathrm{MHz}\right) \delta 169.0,135.6(\mathrm{q}, J=3.5 \mathrm{~Hz}), 133.9(\mathrm{q}, J=28.0 \mathrm{~Hz})$, $124.4(\mathrm{q}, J=277.0 \mathrm{~Hz}), 61.4(\mathrm{~d}, J=2.6 \mathrm{~Hz}), 23.5(\mathrm{q}, J=2.6 \mathrm{~Hz}), 16.4(\mathrm{~d}, J=3.6 \mathrm{~Hz})$, $14.1(\mathrm{~d}, J=3.7 \mathrm{~Hz}), 13.7(\mathrm{~d}, J=3.5 \mathrm{~Hz}) .{ }^{19} \mathrm{~F} \mathrm{NMR}\left(\mathrm{CDCl}_{3}, 376 \mathrm{MHz}\right) \delta-59.8(\mathrm{~s})$. HRMS-ESI calcd for $[\mathrm{M}+\mathrm{Na}]^{+}: 233.0765$, found: 233.0738

\section{Detailed optimization studies}

Table S1. Evaluation of N,P-iridium catalysts in the asymmetric hydrogenation of $1 \mathbf{a}^{\mathrm{a}}$
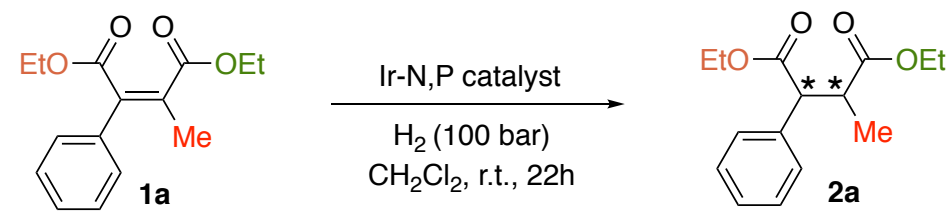

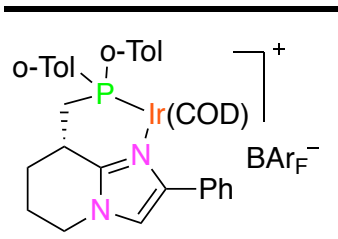

Conv. 99\% $22 \%$ ee

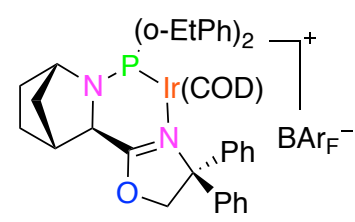

Conv. 29\% $92 \%$ ee

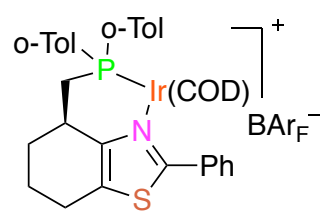

Conv. 37\%

$15 \%$ ee

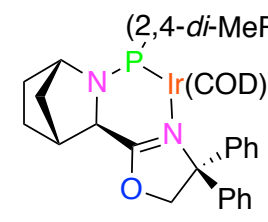

Conv. 78\% 95\% ee

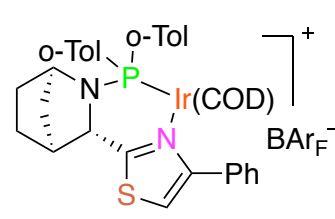

Conv. 68\% $72 \%$ ee

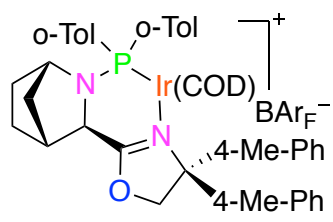

Conv. 95\% $94 \%$ ee

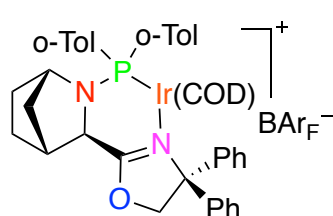

Conv. 30\% $96 \%$ ee

Conv. 99\% $96 \%$ ee

a Reaction conditions: $0.05 \mathrm{mmol}$ substrate, $0.5 \mathrm{~mol} \%$ catalyst, $0.5 \mathrm{~mL} \mathrm{CH}_{2} \mathrm{Cl}_{2}$. Conversions were determined by ${ }^{1} \mathrm{H}-\mathrm{NMR}$ spectroscopy. Enantiomeric excess determined by HPLC and SFC-HPLC analysis using a chiral stationary phase. 
Table S2. Optimization of hydrogenation of tetra-substituted olefin $1 \mathrm{a}^{\mathrm{a}}$

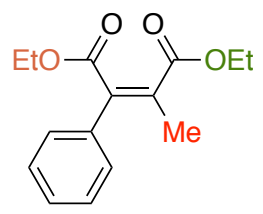

$1 a$

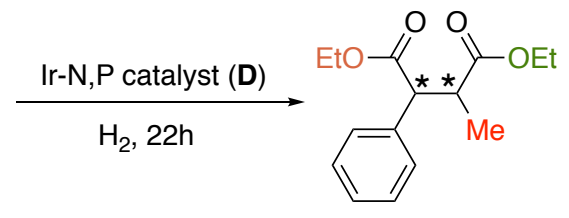

$2 a$

\begin{tabular}{ccccccc}
\hline Entry & $\mathbf{H}_{2}$ (bar) & Solvent & Temp $\left({ }^{\circ} \mathrm{C}\right)$ & Catalyst (mol\%) & Conversion (\%) & ee (\%) \\
\hline & & & & & & \\
1. & 100 & $\mathrm{CH}_{2} \mathrm{Cl}_{2}$ & r.t. & 0.5 & 30 & 96 \\
2. & 100 & $\mathrm{CH}_{2} \mathrm{Cl}_{2}$ & 40 & 0.5 & 78 & 93 \\
3. & 100 & $\mathrm{CH}_{2} \mathrm{Cl}_{2}$ & r.t. & 1.0 & 80 & 96 \\
4. & 100 & $\mathrm{Benzene}^{2}$ & r.t. & 1.0 & 99 & 96 \\
5. & 50 & Benzene & r.t. & 1.0 & 59 & 95 \\
\hline
\end{tabular}

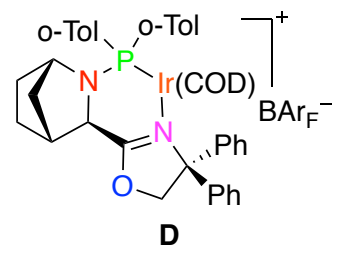

${ }^{a}$ Reaction conditions: $0.05 \mathrm{mmol}$ substrate, $0.5 \mathrm{~mL}$ solvent. Conversions were determined by ${ }^{1} \mathrm{H}-\mathrm{NMR}$ spectroscopy. ${ }^{b}$ Enantiomeric excess determined by HPLC and SFC-HPLC analysis using a chiral stationary phase.

Table S3. Optimization of reaction conditions for the hydrogenation of tetrasubstituted maleate olefins ${ }^{\mathrm{a}}$ 

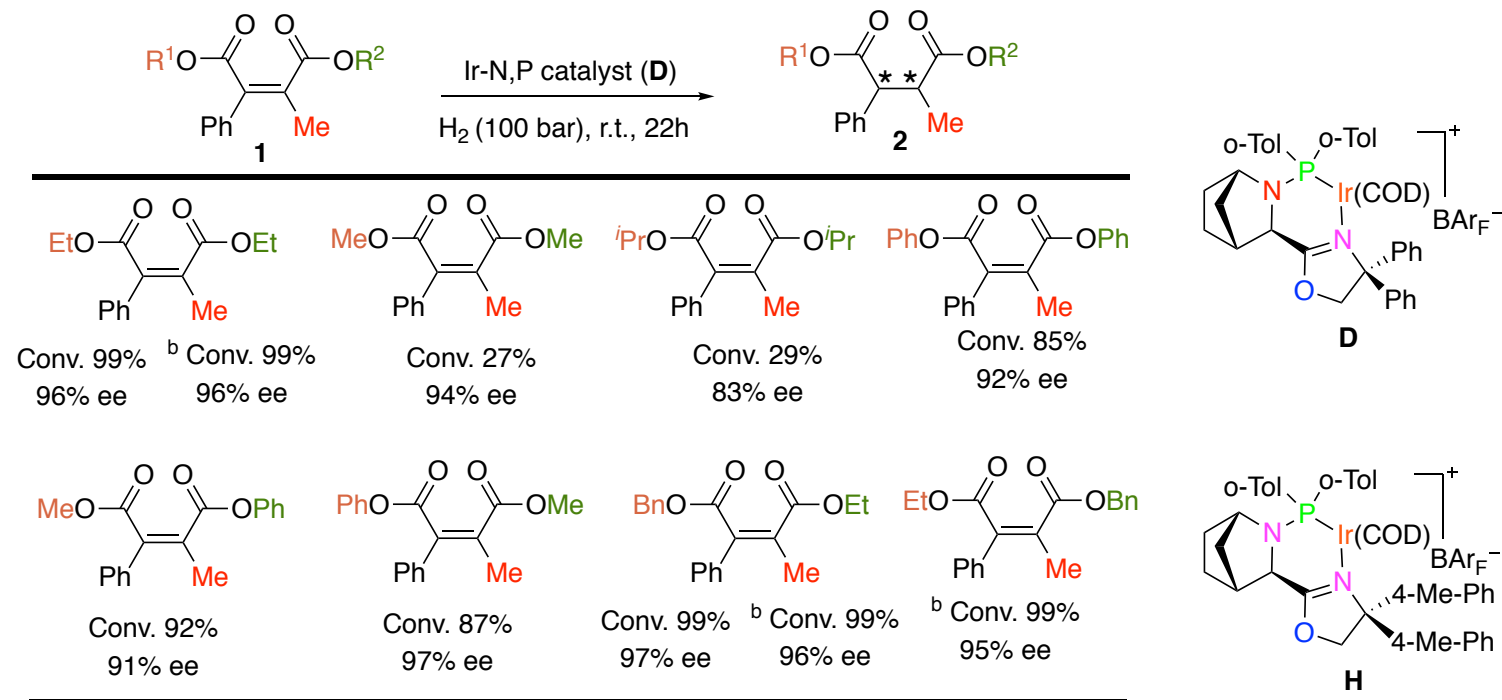

${ }^{\mathrm{a}}$ Reaction conditions: $0.05 \mathrm{mmol}$ substrate, $1.0 \mathrm{~mol} \%$ catalyst, $0.5 \mathrm{~mL}$ benzene. ${ }^{\mathrm{b}} 0.5$ mol\% catalyst $\mathbf{H}, 0.5 \mathrm{~mL} \mathrm{CH} \mathrm{Cl}_{2}$. Conversion determined by ${ }^{1} \mathrm{H}-\mathrm{NMR}$ spectroscopy. Enantiomeric excess determined by HPLC and SFC-HPLC analysis using a chiral stationary phase.

\section{Table S4. Optimization of hydrogenation of tetra-substituted olefin $5 \mathrm{a}^{\mathrm{a}}$}

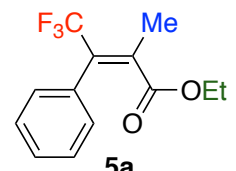

$5 a$

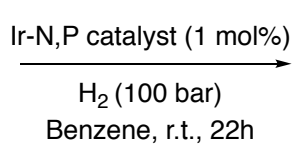

Benzene, r.t., 22h

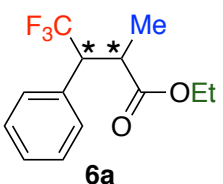

$6 a$

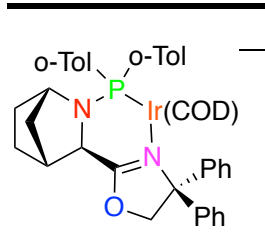

Conv. 19\% 97:3 dr, 43\% ee<smiles></smiles>

Conv. 9\% $90: 10 \mathrm{dr}, 68 \%$ ee

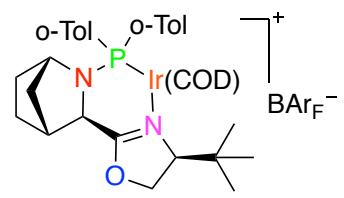

Conv. $20 \%$ 93:7 dr, 72\% ee

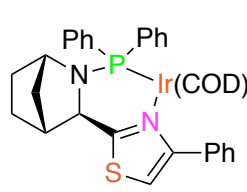

Conv. $20 \%$ $92: 8$ dr, $81 \%$ ee

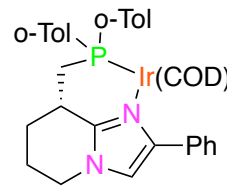

Conv. $66 \%$ $98: 2$ dr, $89 \%$ ee

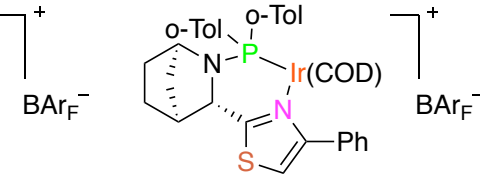

Conv. $53 \%$ $97: 3 \mathrm{dr}, 85 \%$ ee

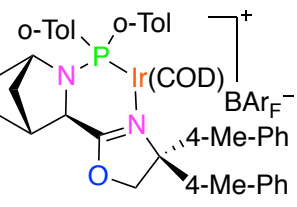
H 
Table S5. Optimization of solvent for hydrogenation of tetra-substituted olefin $5 \mathrm{a}^{\mathrm{a}}$

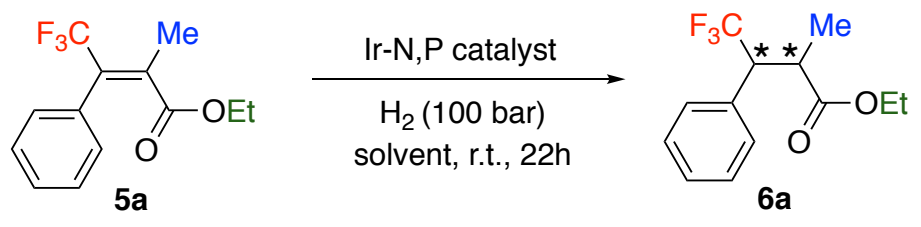

\begin{tabular}{ccccc}
\hline Entry & Solvent & Conversion (\%) & dr (\%) & ee (\%) \\
\hline 1. & Benzene & 87 & $97: 3$ & 93 \\
2. & Toluene & 87 & $98: 2$ & 95 \\
3. & $\mathrm{PhCF}_{3}$ & 99 & $99: 1$ & 95 \\
4. & $\mathrm{CH}_{2} \mathrm{Cl}_{2}$ & 99 & $>99: 1$ & 97 \\
\hline
\end{tabular}

a'Reaction conditions: $0.05 \mathrm{mmol}$ substrate, $0.5 \mathrm{~mL}$ solvent. Conversion determined by ${ }^{1} \mathrm{H}-\mathrm{NMR}$ spectroscopy. Enantiomeric excess determined by SFC-HPLC analysis using a chiral stationary phase.

\section{General procedure for hydrogenation reaction}

A well-dried glass vial was charged with substrate $(0.05$ or $0.5 \mathrm{mmol})$ and Ir-complex (1 mol\%). Freshly distilled $\mathrm{CH}_{2} \mathrm{Cl}_{2}(0.5 \mathrm{~mL}$ or $2 \mathrm{~mL})$ was added to it and placed in a highpressure hydrogenation apparatus. The reactor was purged three times with Ar and three times with $\mathrm{H}_{2}$, and then filled with 100 bar $\mathrm{H}_{2}$ pressure. The reaction was stirred at room temperature for further $22 \mathrm{~h}$ before releasing the $\mathrm{H}_{2}$ pressure. The solvent was removed and the product was dissolved in $\mathrm{Et}_{2} \mathrm{O}: \mathrm{n}$-Pentane $(1: 1,1 \mathrm{~mL})$. The solution was filtered through a short pad of silica and washed with $\mathrm{Et}_{2} \mathrm{O}: \mathrm{n}$-Pentane $(1: 1,1 \mathrm{~mL})$ two times. Conversions were determined by ${ }^{1} \mathrm{H}$ NMR and $e e$ values of the hydrogenated products were determined by using chiral GC or SFC or HPLC.

The fully characterized of the new hydrogenated products are available below.

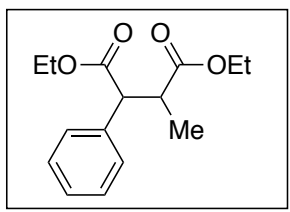

\section{Diethyl 2-methyl-3-phenylsuccinate (2a)}

Yellow oil, 99\% yield. ${ }^{1} \mathrm{H}$ NMR $\left(\mathrm{CDCl}_{3}, 400 \mathrm{MHz}\right): \delta 7.37-7.21(\mathrm{~m}, 5 \mathrm{H}), 4.22-4.03$ $(\mathrm{m}, 2 \mathrm{H}), 3.86(\mathrm{q}, J=7.1 \mathrm{~Hz}, 2 \mathrm{H}), 3.77(\mathrm{~d}, J=11.0 \mathrm{~Hz}, 1 \mathrm{H}), 3.23(\mathrm{~m}, 1 \mathrm{H}), 1.29(\mathrm{~d}, J=$ $6.9 \mathrm{~Hz}, 3 \mathrm{H}), 1.21(\mathrm{t}, J=7.1 \mathrm{~Hz}, 3 \mathrm{H}), 0.94(\mathrm{t}, J=7.1 \mathrm{~Hz}, 3 \mathrm{H}) .{ }^{13} \mathrm{C}$ NMR $\left(\mathrm{CDCl}_{3}, 100\right.$ MHz): $\delta 174.3,172.4,137.0,128.6,128.5,127.7,61.1,60.4,55.2,43.9,16.5,14.2,14.0$. HRMS-ESI calcd for $[\mathrm{M}+\mathrm{Na}]^{+}: 287.1254$, found: 287.1256 . 


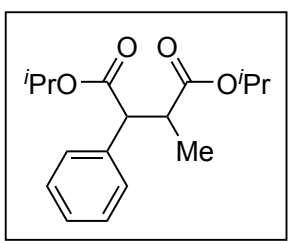

\section{Diisopropyl 2-methyl-3-phenylsuccinate}

Colourless oil, $44 \%$ yield. ${ }^{1} \mathrm{H}$ NMR $\left(\mathrm{CDCl}_{3}, 400 \mathrm{MHz}\right): \delta 7.40$ - 7.32 (m, 2H), 7.34 - 7.21 (m, 3H), 5.01 (hept, $J=6.3 \mathrm{~Hz}, 1 \mathrm{H}$ ), 4.74 (hept, $J=6.3 \mathrm{~Hz}, 1 \mathrm{H}), 3.73$ (d, $J=11.1 \mathrm{~Hz}$, $1 \mathrm{H}), 3.22(\mathrm{dq}, J=11.2,6.8 \mathrm{~Hz}, 1 \mathrm{H}), 1.30(\mathrm{~d}, J=6.8 \mathrm{~Hz}, 3 \mathrm{H}), 1.25(\mathrm{~d}, J=6.3 \mathrm{~Hz}, 3 \mathrm{H})$, $1.15(\mathrm{~d}, J=6.3 \mathrm{~Hz}, 3 \mathrm{H}), 1.03(\mathrm{~d}, J=6.3 \mathrm{~Hz}, 3 \mathrm{H}), 0.85(\mathrm{~d}, J=6.2 \mathrm{~Hz}, 3 \mathrm{H}) .{ }^{13} \mathrm{C}$ NMR $\left(\mathrm{CDCl}_{3}, 100 \mathrm{MHz}\right): \delta 173.8,171.9,137.1,128.6,128.5,127.6,68.5,67.6,55.6,43.9$, 21.9, 21.6, 21.6, 21.4, 16.5. HRMS-ESI calcd for [M+Na $]^{+}: 315.1567$, found: 315.1557 .

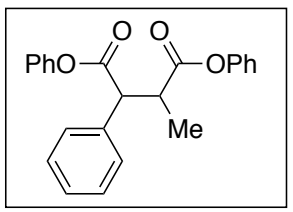

\section{Diphenyl 2-methyl-3-phenylsuccinate}

Yellow oil, 99\% yield. ${ }^{1} \mathrm{H}$ NMR $\left(\mathrm{CDCl}_{3}, 400 \mathrm{MHz}\right): \delta 7.56-7.50(\mathrm{~m}, 2 \mathrm{H}), 7.44-7.32$ (m, 5H), $7.29-7.19(\mathrm{~m}, 3 \mathrm{H}), 7.19-7.13(\mathrm{~m}, 1 \mathrm{H}), 7.01(\mathrm{dd}, J=8.6,1.2 \mathrm{~Hz}, 2 \mathrm{H}), 6.61$ (dd, $J=8.5,1.2 \mathrm{~Hz}, 2 \mathrm{H}), 4.17(\mathrm{~d}, J=10.8 \mathrm{~Hz}, 1 \mathrm{H}), 3.61(\mathrm{~m}, 1 \mathrm{H}), 1.58(\mathrm{~d}, J=6.8 \mathrm{~Hz}$, $3 \mathrm{H}) .{ }^{13} \mathrm{C}$ NMR $\left(\mathrm{CDCl}_{3}, 100 \mathrm{MHz}\right): \delta 172.8,170.8,150.7,150.4,136.2,129.6,129.5$, 129.1, 128.9, 128.4, 126.2, 126.0, 121.5, 121.4, 55.2, 44.0, 16.6. HRMS-ESI calcd for $[\mathrm{M}+\mathrm{Na}]^{+}: 383.1254$, found: 383.1271 .

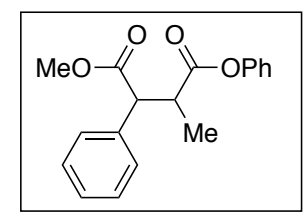

\section{1-Methyl 4-phenyl 3-methyl-2-phenylsuccinate}

Yellow oil, 99\% yield. ${ }^{1} \mathrm{H}$ NMR $\left(\mathrm{CDCl}_{3}, 400 \mathrm{MHz}\right): \delta 7.44-7.40(\mathrm{~m}, 2 \mathrm{H}), 7.39-7.30$ (m, 3H), $7.24(\mathrm{dd}, J=8.6,6.9 \mathrm{~Hz}, 2 \mathrm{H}), 7.17-7.10(\mathrm{~m}, 1 \mathrm{H}), 6.58-6.52(\mathrm{~m}, 2 \mathrm{H}), 3.90(\mathrm{~d}$, $J=11.0 \mathrm{~Hz}, 1 \mathrm{H}), 3.71(\mathrm{~s}, 3 \mathrm{H}), 3.52(\mathrm{~m}, 1 \mathrm{H}), 1.45(\mathrm{~d}, J=6.8 \mathrm{~Hz}, 3 \mathrm{H}) .{ }^{13} \mathrm{C} \mathrm{NMR}\left(\mathrm{CDCl}_{3}\right.$, $100 \mathrm{MHz}): \delta 172.9,172.7,150.4,136.6,129.4,128.9,128.8,128.2,125.9,121.4,55.2$, 52.5, 44.0, 16.7. HRMS-ESI calcd for [M+Na] $]^{+}: 321.1097$, found: 321.1092 .

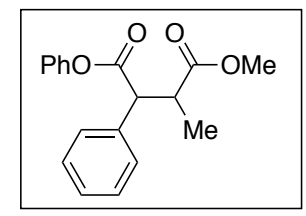

\section{1-Methyl 4-phenyl 2-methyl-3-phenylsuccinate}

Yellow oil, $15 \%$ yield. ${ }^{1} \mathrm{H}$ NMR $\left(\mathrm{CDCl}_{3}, 400 \mathrm{MHz}\right): \delta 7.45-7.40(\mathrm{~m}, 2 \mathrm{H}), 7.39-7.28$ (m, 5H), $7.25-7.17(\mathrm{~m}, 1 \mathrm{H}), 7.02-6.96(\mathrm{~m}, 2 \mathrm{H}), 4.08(\mathrm{~d}, J=10.5 \mathrm{~Hz}, 1 \mathrm{H}), 3.48(\mathrm{~s}$, $3 \mathrm{H}), 3.40-3.30(\mathrm{~m}, 1 \mathrm{H}), 1.42(\mathrm{dd}, J=6.8,0.6 \mathrm{~Hz}, 3 \mathrm{H}) .{ }^{13} \mathrm{C} \mathrm{NMR}\left(\mathrm{CDCl}_{3}, 100 \mathrm{MHz}\right)$ : 
$\delta 174.5,170.9,150.7,136.3,129.5,128.8,128.5,128.0,126.1,121.4,54.9,51.8,43.8$, 16.4. HRMS-ESI calcd for $[\mathrm{M}+\mathrm{Na}]^{+}: 321.1097$, found: 321.1110 .

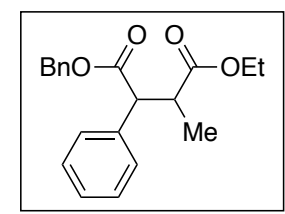

\section{1-Benzyl 4-ethyl 3-methyl-2-phenylsuccinate (2b)}

Colourless oil, 99\% yield. ${ }^{1} \mathrm{H}$ NMR $\left(\mathrm{CDCl}_{3}, 400 \mathrm{MHz}\right): \delta 7.39-7.18(\mathrm{~m}, 10 \mathrm{H}), 5.16(\mathrm{~d}$, $J=12.4 \mathrm{~Hz}, 1 \mathrm{H}), 5.07(\mathrm{~d}, J=12.5 \mathrm{~Hz}, 1 \mathrm{H}), 3.91-3.82(\mathrm{~m}, 3 \mathrm{H}), 3.31-3.21(\mathrm{~m}, 1 \mathrm{H})$, $1.27(\mathrm{~d}, J=6.8 \mathrm{~Hz}, 3 \mathrm{H}), 0.94(\mathrm{t}, J=7.1 \mathrm{~Hz}, 3 \mathrm{H}) .{ }^{13} \mathrm{C} \mathrm{NMR}\left(\mathrm{CDCl}_{3}, 100 \mathrm{MHz}\right): \delta 174.1$, $172.2,136.7,135.8,128.5(9), 128.6$ (2C), 128.3, 128.0, 127.8, 66.8, 60.4, 55.2, 43.8, 16.5, 13.9. HRMS-ESI calcd for $[\mathrm{M}+\mathrm{Na}]^{+}: 349.1410$, found: 349.1394 .

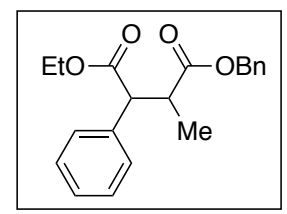

\section{1-Benzyl 4-ethyl 2-methyl-3-phenylsuccinate (2c)}

Colourless oil, 99\% yield. ${ }^{1} \mathrm{H}$ NMR $\left(\mathrm{CDCl}_{3}, 400 \mathrm{MHz}\right): \delta 7.39-7.22(\mathrm{~m}, 8 \mathrm{H}), 7.09-$ $7.00(\mathrm{~m}, 2 \mathrm{H}), 4.88(\mathrm{~s}, 2 \mathrm{H}), 4.26-4.05(\mathrm{~m}, 2 \mathrm{H}), 3.83(\mathrm{~d}, J=11.0 \mathrm{~Hz}, 1 \mathrm{H}), 3.34(\mathrm{dq}, J=$

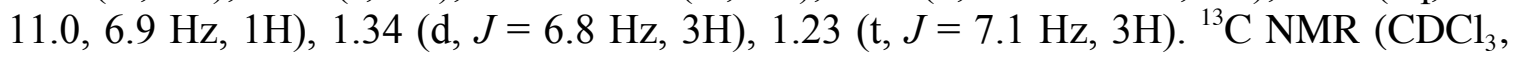
$100 \mathrm{MHz}): \delta 174.2,172.3,136.9,135.7,128.7,128.5,128.5,128.2,128.1,127.8,66.3$, 61.2, 55.1, 44.0, 16.6, 14.2. HRMS-ESI calcd for [M+Na] $]^{+}: 349.1416$, found: 349.1420 .

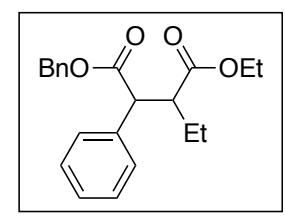

\section{1-Benzyl 4-ethyl 3-ethyl-2-phenylsuccinate (2d)}

Colourless oil, $99 \%$ yield. ${ }^{1} \mathrm{H}$ NMR $\left(\mathrm{CDCl}_{3}, 400 \mathrm{MHz}\right): \delta 7.37-7.20(\mathrm{~m}, 10 \mathrm{H}), 5.15(\mathrm{~d}$, $J=12.5 \mathrm{~Hz}, 1 \mathrm{H}), 5.05(\mathrm{~d}, J=12.4 \mathrm{~Hz}, 1 \mathrm{H}), 3.87-3.81$ (m, 3H), 3.15 (ddd, $J=11.5,9.7$, $4.4 \mathrm{~Hz}, 1 \mathrm{H}), 1.76-1.59(\mathrm{~m}, 2 \mathrm{H}), 0.91$ (q, $J=7.3 \mathrm{~Hz}, 6 \mathrm{H}) .{ }^{13} \mathrm{C} \mathrm{NMR}\left(\mathrm{CDCl}_{3}, 100 \mathrm{MHz}\right)$ : $\delta 173.4,172.3,136.6,135.8,128.7,128.6$ (2C), 128.3, 128.0, 127.9, 66.85, 60.3, 54.5, 51.3, 25.0, 14.0, 11.9. HRMS-ESI calcd for $[\mathrm{M}+\mathrm{Na}]^{+}: 363.1567$, found: 363.1563 .

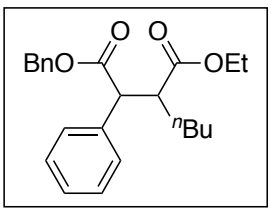

1-Benzyl 4-ethyl 3-butyl-2-phenylsuccinate (2e) 
White solid, 99\% yield. ${ }^{1} \mathrm{H}$ NMR $\left(\mathrm{CDCl}_{3}, 400 \mathrm{MHz}\right): \delta 7.39-7.16(\mathrm{~m}, 10 \mathrm{H}), 5.16(\mathrm{~d}, J=$ $12.4 \mathrm{~Hz}, 1 \mathrm{H}), 5.06(\mathrm{~d}, J=12.4 \mathrm{~Hz}, 1 \mathrm{H}), 3.85-3.79(\mathrm{~m}, 3 \mathrm{H}), 3.20$ (ddd, $J=11.5,10.5$, $3.6 \mathrm{~Hz}, 1 \mathrm{H}), 1.76-1.47(\mathrm{~m}, 1 \mathrm{H}), 1.36-1.20(\mathrm{~m}, 5 \mathrm{H}), 0.93-0.81(\mathrm{~m}, 6 \mathrm{H}) .{ }^{13} \mathrm{C} \mathrm{NMR}$ $\left(\mathrm{CDCl}_{3}, 100 \mathrm{MHz}\right): \delta 173.6,172.3,136.5,135.8,128.7,128.6,128.6,128.3,128.1$, $127.8,66.8,60.2,54.7,49.8,31.5,29.6,22.5,14.0,13.9$. HRMS-ESI calcd for $[\mathrm{M}+\mathrm{Na}]^{+}$: 391.1880, found: 391.1888 .

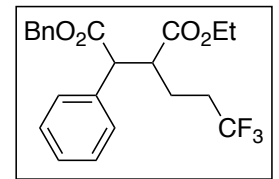

\section{1-Benzyl 4-ethyl 2-phenyl-3-(3,3,3-trifluoropropyl)succinate(2f)}

Colourless oil, $98 \%$ yield. ${ }^{1} \mathrm{H}$ NMR $\left(\mathrm{CDCl}_{3}, 400 \mathrm{MHz}\right): \delta 7.39-7.16(\mathrm{~m}, 10 \mathrm{H}), 5.23-$ $5.00(\mathrm{~m}, 2 \mathrm{H}), 3.91-3.80(\mathrm{~m}, 3 \mathrm{H}), 3.29-3.16(\mathrm{~m}, 1 \mathrm{H}), 2.19-2.04(\mathrm{~m}, 2 \mathrm{H}), 2.00-1.80$ $(\mathrm{m}, 2 \mathrm{H}), 0.90(\mathrm{t}, J=7.1 \mathrm{~Hz}, 3 \mathrm{H}) .{ }^{13} \mathrm{C} \mathrm{NMR}\left(\mathrm{CDCl}_{3}, 100 \mathrm{MHz}\right): \delta 172.4,171.8,135.8$, $135.5,128.7,128.7,128.6,128.5,128.1,67.2,60.8,54.2,48.6,31.9$ (q, $J=29.2 \mathrm{~Hz}$ ), $23.8(\mathrm{q}, J=3.0 \mathrm{~Hz}), 13.9 .{ }^{19} \mathrm{~F} \mathrm{NMR}\left(\mathrm{CDCl}_{3}, 376 \mathrm{MHz}\right) \delta-66.6$ (s). HRMS-ESI calcd for $[\mathrm{M}+\mathrm{Na}]^{+}: 431.1446$, found: 431.1449 .

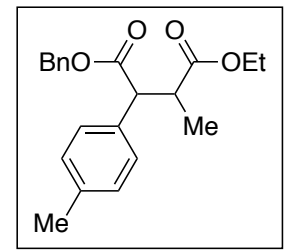

\section{1-Benzyl 4-ethyl 3-methyl-2-(p-tolyl)succinate (2g)}

White solid, 99\% yield. ${ }^{1} \mathrm{H}$ NMR $\left(\mathrm{CDCl}_{3}, 400 \mathrm{MHz}\right): \delta 7.36-7.28(\mathrm{~m}, 3 \mathrm{H}), 7.26-7.19$ $(\mathrm{m}, 5 \mathrm{H}), 7.11-7.05(\mathrm{~m}, 2 \mathrm{H}), 5.16(\mathrm{~d}, J=12.4 \mathrm{~Hz}, 1 \mathrm{H}), 5.05(\mathrm{~d}, J=12.4 \mathrm{~Hz}, 1 \mathrm{H}), 3.88$ $(\mathrm{q}, J=7.1 \mathrm{~Hz}, 2 \mathrm{H}), 3.82(\mathrm{~d}, J=10.9 \mathrm{~Hz}, 1 \mathrm{H}), 3.23(\mathrm{dq}, J=10.9,6.8 \mathrm{~Hz}, 1 \mathrm{H}), 2.30$ (s, $3 \mathrm{H}), 1.25(\mathrm{~d}, J=6.8 \mathrm{~Hz}, 3 \mathrm{H}), 0.97(\mathrm{t}, J=7.1 \mathrm{~Hz}, 3 \mathrm{H}) .{ }^{13} \mathrm{C} \mathrm{NMR}\left(\mathrm{CDCl}_{3}, 100 \mathrm{MHz}\right): \delta$ $174.2,172.4,137.5,135.9,133.7,129.3,128.6,128.4,128.3,128.1,66.8,60.4,54.8$, 43.9, 21.2, 16.5, 14.0. HRMS-ESI calcd for $[\mathrm{M}+\mathrm{Na}]^{+}: 363.1567$, found: 363.1549 .

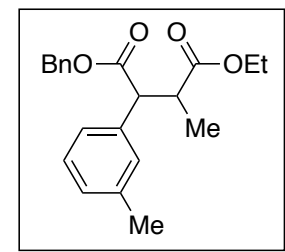

\section{1-Benzyl 4-ethyl 3-methyl-2-(m-tolyl)succinate (2h)}

Colourless oil, 99\% yield. ${ }^{1} \mathrm{H}$ NMR $\left(\mathrm{CDCl}_{3}, 400 \mathrm{MHz}\right): \delta 7.37-7.21(\mathrm{~m}, 6 \mathrm{H}), 7.20-$ $7.09(\mathrm{~m}, 3 \mathrm{H}), 7.05$ (ddt, $J=6.8,1.6,0.8 \mathrm{~Hz}, 1 \mathrm{H}), 5.17(\mathrm{~d}, J=12.4 \mathrm{~Hz}, 1 \mathrm{H}), 5.06(\mathrm{~d}, J=$ $12.4 \mathrm{~Hz}, 1 \mathrm{H}), 3.87$ (d, $J=7.1 \mathrm{~Hz}, 2 \mathrm{H}), 3.82(\mathrm{~d}, J=10.9 \mathrm{~Hz}, 1 \mathrm{H}), 3.24$ (dq, $J=10.9,6.8$ $\mathrm{Hz}, 1 \mathrm{H}), 2.30(\mathrm{~s}, 3 \mathrm{H}), 1.26(\mathrm{~d}, J=6.8 \mathrm{~Hz}, 3 \mathrm{H}), 0.95(\mathrm{~d}, J=7.1 \mathrm{~Hz}, 3 \mathrm{H}) .{ }^{13} \mathrm{C} \mathrm{NMR}$ $\left(\mathrm{CDCl}_{3}, 100 \mathrm{MHz}\right): \delta 174.2,172.3,138.2,136.6,135.9,129.3,128.6,128.6,128.5$, 
$128.3,128.1,125.6,66.8,60.4,55.1,43.8,21.5,16.5,14.0$. HRMS-ESI calcd for $[\mathrm{M}+\mathrm{Na}]^{+}: 363.1567$, found: 363.1584 .

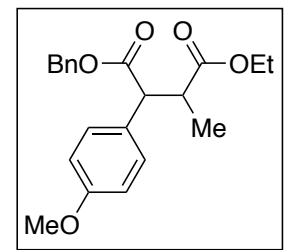

\section{1-Benzyl 4-ethyl 2-(4-methoxyphenyl)-3-methylsuccinate (2i)}

White solid, 98\% yield. ${ }^{1} \mathrm{H}$ NMR $\left(\mathrm{CDCl}_{3}, 400 \mathrm{MHz}\right): \delta 7.35-7.23(\mathrm{~m}, 7 \mathrm{H}), 6.86-6.77$ $(\mathrm{m}, 2 \mathrm{H}), 5.15(\mathrm{~d}, J=12.4 \mathrm{~Hz}, 1 \mathrm{H}), 5.06(\mathrm{~d}, J=12.4 \mathrm{~Hz}, 1 \mathrm{H}), 3.88(\mathrm{q}, J=7.1 \mathrm{~Hz}, 2 \mathrm{H})$, $3.80(\mathrm{~d}, J=11.1 \mathrm{~Hz}, 1 \mathrm{H}), 3.78(\mathrm{~s}, 3 \mathrm{H}), 3.21(\mathrm{dq}, J=11.0,6.8 \mathrm{~Hz}, 1 \mathrm{H}), 1.25(\mathrm{~d}, J=6.8$ $\mathrm{Hz}, 3 \mathrm{H}), 0.97$ (t, $J=7.1 \mathrm{~Hz}, 3 \mathrm{H}) .{ }^{13} \mathrm{C}$ NMR $\left(\mathrm{CDCl}_{3}, 100 \mathrm{MHz}\right): \delta 174.3,172.5,159.2$, 135.9, 129.7, 128.8, 128.6, 128.3, 128.1, 114.0, 66.8, 60.5, 55.4, 54.4, 43.9, 16.5, 14.1 . HRMS-ESI calcd for $[\mathrm{M}+\mathrm{Na}]^{+}: 379.1516$, found: 379.1516 .

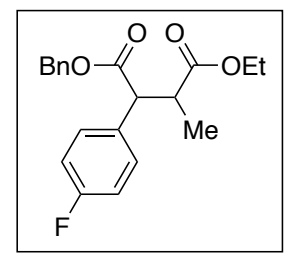

\section{1-Benzyl 4-ethyl 2-(4-fluorophenyl)-3-methylsuccinate (2j)}

Colourless oil, 99\% yield. ${ }^{1} \mathrm{H}$ NMR $\left(\mathrm{CDCl}_{3}, 400 \mathrm{MHz}\right): \delta 7.35-7.27(\mathrm{~m}, 5 \mathrm{H}), 7.26-$ $7.21(\mathrm{~m}, 2 \mathrm{H}), 7.01-6.93(\mathrm{~m}, 2 \mathrm{H}), 5.15(\mathrm{~d}, J=12.4 \mathrm{~Hz}, 1 \mathrm{H}), 5.07(\mathrm{~d}, J=12.4 \mathrm{~Hz}, 1 \mathrm{H})$, $3.88(\mathrm{q}, J=7.1 \mathrm{~Hz}, 2 \mathrm{H}), 3.83(\mathrm{~d}, J=11.0 \mathrm{~Hz}, 1 \mathrm{H}), 3.21(\mathrm{dq}, J=11.0,6.8 \mathrm{~Hz}, 1 \mathrm{H}), 1.25$ $(\mathrm{d}, J=6.8 \mathrm{~Hz}, 3 \mathrm{H}), 0.97(\mathrm{t}, J=7.1 \mathrm{~Hz}, 3 \mathrm{H}) .{ }^{13} \mathrm{C} \mathrm{NMR}\left(\mathrm{CDCl}_{3}, 100 \mathrm{MHz}\right): \delta 174.0$, $172.1,162.5(\mathrm{~d}, J=246.3 \mathrm{~Hz}), 135.7,132.6(\mathrm{~d}, J=3.3 \mathrm{~Hz}), 130.3(\mathrm{~d}, J=8.1 \mathrm{~Hz}), 128.7$, 128.4, 128.1, 115.6, 115.4, 67.0, 60.6, 54.4, 44.0, 16.5, 14.0. ${ }^{19} \mathrm{~F} \mathrm{NMR}\left(\mathrm{CDCl}_{3}, 376\right.$ MHz) $\delta-62.7$ (s). HRMS-ESI calcd for $[\mathrm{M}+\mathrm{Na}]^{+}: 367.1316$, found: 367.1316.

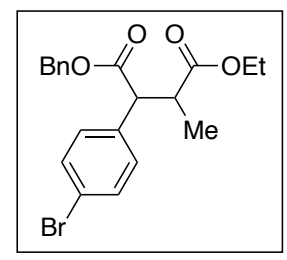

\section{1-Benzyl 4-ethyl 2-(4-bromophenyl)-3-methylsuccinate (2k)}

Colourless oil, 94\% yield. ${ }^{1} \mathrm{H}$ NMR $\left(\mathrm{CDCl}_{3}, 400 \mathrm{MHz}\right): \delta 7.44-7.39(\mathrm{~m}, 2 \mathrm{H}), 7.35-$ $7.29(\mathrm{~m}, 3 \mathrm{H}), 7.26-7.19(\mathrm{~m}, 4 \mathrm{H}), 5.15(\mathrm{~d}, J=12.3 \mathrm{~Hz}, 1 \mathrm{H}), 5.06(\mathrm{~d}, J=12.4 \mathrm{~Hz}, 1 \mathrm{H})$, 3.89 (q, $J=7.1 \mathrm{~Hz}, 2 \mathrm{H}), 3.81(\mathrm{~d}, J=11.0 \mathrm{~Hz}, 1 \mathrm{H}), 3.20(\mathrm{dq}, J=11.0,6.9 \mathrm{~Hz}, 1 \mathrm{H}), 1.25$ (d, $J=6.9 \mathrm{~Hz}, 3 \mathrm{H}), 0.99$ (t, $J=7.1 \mathrm{~Hz}, 3 \mathrm{H}) .{ }^{13} \mathrm{C} \mathrm{NMR}\left(\mathrm{CDCl}_{3}, 100 \mathrm{MHz}\right): \delta 173.9$, $171.8,135.8,135.6,131.8,130.3,128.7,128.5,128.2,122.0,67.1,60.6,54.6,43.7,16.5$, 14.0. HRMS-ESI calcd for $[\mathrm{M}+\mathrm{Na}]^{+}: 427.0515$, found: $427.0417,429.0500[\mathrm{M}+\mathrm{Na}+2]^{+}$. 


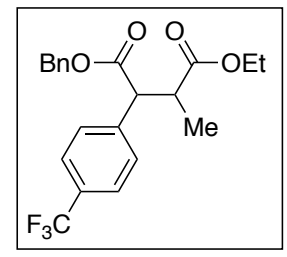

1-Benzyl 4-ethyl 3-methyl-2-(4-(trifluoromethyl)phenyl)succinate (2l)

Yellow oil, 99\% yield. ${ }^{1} \mathrm{H}$ NMR $\left(\mathrm{CDCl}_{3}, 400 \mathrm{MHz}\right): \delta 7.57(\mathrm{~d}, J=8.2 \mathrm{~Hz}, 2 \mathrm{H}), 7.49(\mathrm{~d}, J$ $=8.1 \mathrm{~Hz}, 2 \mathrm{H}), 7.38-7.30(\mathrm{~m}, 3 \mathrm{H}), 7.30-7.20(\mathrm{~m}, 2 \mathrm{H}), 5.18(\mathrm{~d}, J=12.3 \mathrm{~Hz}, 1 \mathrm{H}), 5.11$ $(\mathrm{d}, J=12.3 \mathrm{~Hz}, 1 \mathrm{H}), 3.98-3.86(\mathrm{~m}, 3 \mathrm{H}), 3.29$ (dq, $J=10.9,6.8 \mathrm{~Hz}, 1 \mathrm{H}), 1.31$ (d, $J=6.8$ $\mathrm{Hz}, 3 \mathrm{H}), 0.98$ (t, $J=7.1 \mathrm{~Hz}, 3 \mathrm{H}) .{ }^{13} \mathrm{C} \mathrm{NMR}\left(\mathrm{CDCl}_{3}, 100 \mathrm{MHz}\right): \delta 173.8,171.5,140.8$, $135.5,129.0,128.7,128.5,128.2,125.6,125.5,125.5,67.2,60.7,54.9,43.7,16.5,13.9$. ${ }^{19} \mathrm{~F}$ NMR $\left(\mathrm{CDCl}_{3}, 376 \mathrm{MHz}\right) \delta-114.7$ (s). HRMS-ESI calcd for $[\mathrm{M}+\mathrm{Na}]^{+}: 417.1284$, found: 417.1281.

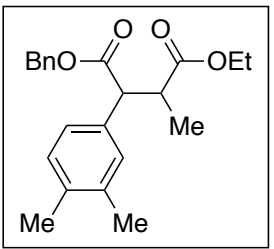

\section{1-Benzyl 4-ethyl 2-(3,4-dimethylphenyl)-3-methylsuccinate (2m)}

Yellow oil, 96\% yield. ${ }^{1} \mathrm{H}$ NMR $\left(\mathrm{CDCl}_{3}, 400 \mathrm{MHz}\right): \delta 7.35-7.22(\mathrm{~m}, 5 \mathrm{H}), 7.09-7.01$ $(\mathrm{m}, 3 \mathrm{H}), 5.17(\mathrm{~d}, J=12.4 \mathrm{~Hz}, 1 \mathrm{H}), 5.04(\mathrm{~d}, J=12.4 \mathrm{~Hz}, 1 \mathrm{H}), 3.89$ (q, $J=7.1 \mathrm{~Hz}, 2 \mathrm{H})$, $3.80(\mathrm{~d}, J=10.9 \mathrm{~Hz}, 1 \mathrm{H}), 3.22(\mathrm{dq}, J=10.9,6.9 \mathrm{~Hz}, 1 \mathrm{H}), 2.21(\mathrm{~s}, 6 \mathrm{H}), 1.25$ (d, $J=6.9$ $\mathrm{Hz}, 3 \mathrm{H}), 0.97$ (t, $J=7.1 \mathrm{~Hz}, 3 \mathrm{H}) .{ }^{13} \mathrm{C} \mathrm{NMR}\left(\mathrm{CDCl}_{3}, 100 \mathrm{MHz}\right): \delta 174.3,172.5,136.7$, 136.1, 135.9, 134.1, 129.8, 129.7, 128.6, 128.3, 128.1, 125.9, 66.8, 60.4, 54.7, 43.8, 19.9, $19.5,16.5,14.0$. HRMS-ESI calcd for $[\mathrm{M}+\mathrm{Na}]^{+}: 377.1723$, found: 377.1728 .

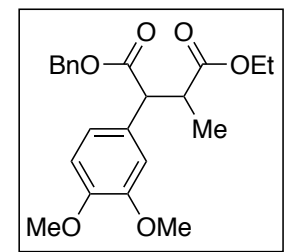

\section{1-Benzyl 4-ethyl 2-(3,4-dimethoxyphenyl)-3-methylsuccinate (2n)}

Colourless oil, 71\% yield. ${ }^{1} \mathrm{H} \mathrm{NMR}\left(\mathrm{CDCl}_{3}, 400 \mathrm{MHz}\right): \delta 7.31(\mathrm{qd}, J=2.9,2.1 \mathrm{~Hz}, 3 \mathrm{H})$, $7.26-7.23(\mathrm{~m}, 2 \mathrm{H}), 6.91-6.82(\mathrm{~m}, 2 \mathrm{H}), 6.77(\mathrm{~d}, J=8.2 \mathrm{~Hz}, 1 \mathrm{H}), 5.23-4.98(\mathrm{~m}, 2 \mathrm{H})$, $3.94-3.73(\mathrm{~m}, 9 \mathrm{H}), 3.22(\mathrm{dq}, J=11.1,6.8 \mathrm{~Hz}, 1 \mathrm{H}), 1.25(\mathrm{~d}, J=6.8 \mathrm{~Hz}, 3 \mathrm{H}), 0.99$ (t, $J=$ $7.1 \mathrm{~Hz}, 3 \mathrm{H}) .{ }^{13} \mathrm{C} \mathrm{NMR}\left(\mathrm{CDCl}_{3}, 100 \mathrm{MHz}\right): \delta 174.3,172.4,148.9,148.7,135.8,129.2$, $128.6,128.4,128.2,121.0,111.5,111.1,66.8,60.5,56.0,56.0,54.7,43.9,16.6,14.1$. HRMS-ESI calcd for $[\mathrm{M}+\mathrm{Na}]^{+}:$409.1627, found: 409.1615 . 


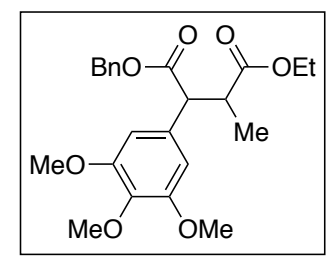

\section{1-Benzyl 4-ethyl 3-methyl-2-(3,4,5-trimethoxyphenyl)succinate(2o)}

Colourless oil, 99\% yield. ${ }^{1} \mathrm{H}$ NMR $\left(\mathrm{CDCl}_{3}, 400 \mathrm{MHz}\right): \delta 7.36-7.27(\mathrm{~m}, 3 \mathrm{H}), 7.28-$ $7.21(\mathrm{~m}, 2 \mathrm{H}), 6.52(\mathrm{~s}, 2 \mathrm{H}), 5.27-4.99(\mathrm{~m}, 2 \mathrm{H}), 3.91(\mathrm{qd}, J=7.1,4.2 \mathrm{~Hz}, 2 \mathrm{H}), 3.81-$ $3.76(\mathrm{~m}, 10 \mathrm{H}), 3.22(\mathrm{dq}, J=11.1,6.8 \mathrm{~Hz}, 1 \mathrm{H}), 1.26(\mathrm{~d}, J=6.8 \mathrm{~Hz}, 3 \mathrm{H}), 0.99(\mathrm{t}, J=7.1$ $\mathrm{Hz}, 3 \mathrm{H}) .{ }^{13} \mathrm{C} \mathrm{NMR}\left(\mathrm{CDCl}_{3}, 100 \mathrm{MHz}\right): \delta 174.2,172.1,153.2,137.6,135.8,132.2,128.7$, 128.4, 128.2, 105.6, 66.9, 61.0, 60.5, 56.2, 55.3, 43.8, 16.6, 14.1. HRMS-ESI calcd for $[\mathrm{M}+\mathrm{Na}]^{+}:$439.1733, found: 439.1736 .

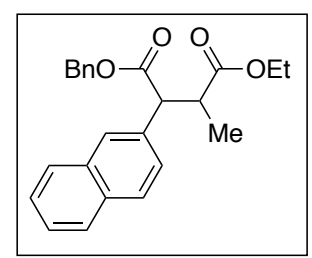

\section{1-Benzyl 4-ethyl 3-methyl-2-(naphthalen-2-yl)succinate (2p)}

White solid, 97\% yield. ${ }^{1} \mathrm{H}$ NMR $\left(\mathrm{CDCl}_{3}, 400 \mathrm{MHz}\right): \delta 7.85-7.74(\mathrm{~m}, 4 \mathrm{H}), 7.52-7.42$ (m, 3H), $7.32-7.19(\mathrm{~m}, 5 \mathrm{H}), 5.18(\mathrm{~d}, J=12.4 \mathrm{~Hz}, 1 \mathrm{H}), 5.07(\mathrm{~d}, J=12.4 \mathrm{~Hz}, 1 \mathrm{H}), 4.05$ $(\mathrm{d}, J=10.9 \mathrm{~Hz}, 1 \mathrm{H}), 3.88-3.76(\mathrm{~m}, 2 \mathrm{H}), 3.44-3.32(\mathrm{~m}, 1 \mathrm{H}), 1.32(\mathrm{~d}, J=6.8 \mathrm{~Hz}, 3 \mathrm{H})$, $0.86(\mathrm{~d}, J=7.1 \mathrm{~Hz}, 3 \mathrm{H}) .{ }^{13} \mathrm{C} \mathrm{NMR}\left(\mathrm{CDCl}_{3}, 100 \mathrm{MHz}\right): \delta 174.2,172.2,135.7,134.2$, 133.4, 133.0, 128.6, 128.4, 128.3, 128.1 (2C), 127.7, 127.7, 126.4, 126.2, 126.1, 66.9, $60.5,55.2,43.8,16.6,13.9$. HRMS-ESI calcd for $[\mathrm{M}+\mathrm{Na}]^{+}: 399.1567$, found: 399.1553 .

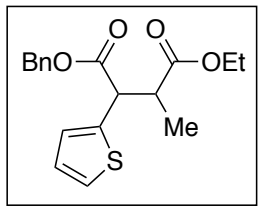

\section{1-Benzyl 4-ethyl 3-methyl-2-(thiophen-2-yl)succinate (2q)}

Yellow oil, 99\% yield. ${ }^{1} \mathrm{H} \mathrm{NMR}\left(\mathrm{CDCl}_{3}, 400 \mathrm{MHz}\right): \delta 7.38-7.28(\mathrm{~m}, 5 \mathrm{H}), 7.20(\mathrm{dd}, J=$ $5.1,1.2 \mathrm{~Hz}, 1 \mathrm{H}), 6.99-6.96(\mathrm{~m}, 1 \mathrm{H}), 6.91(\mathrm{dd}, J=5.1,3.5 \mathrm{~Hz}, 1 \mathrm{H}), 5.18(\mathrm{~d}, J=12.4 \mathrm{~Hz}$, $1 \mathrm{H}), 5.13(\mathrm{~d}, J=12.3 \mathrm{~Hz}, 1 \mathrm{H}), 4.19(\mathrm{~d}, J=10.3 \mathrm{~Hz}, 1 \mathrm{H}), 3.97$ (qd, $J=7.1,1.0 \mathrm{~Hz}, 2 \mathrm{H})$, $3.20(\mathrm{dq}, J=10.3,6.9 \mathrm{~Hz}, 1 \mathrm{H}), 1.24(\mathrm{~d}, J=6.9 \mathrm{~Hz}, 3 \mathrm{H}), 1.06(\mathrm{t}, J=7.1 \mathrm{~Hz}, 3 \mathrm{H}) .{ }^{13} \mathrm{C}$ NMR $\left(\mathrm{CDCl}_{3}, 100 \mathrm{MHz}\right): \delta 173.8,171.4,138.5,135.6,128.7,128.5,128.3,126.8,126.7$, 125.3, 67.2, 60.7, 50.2, 45.0, 16.2, 14.1. HRMS-ESI calcd for $[\mathrm{M}+\mathrm{Na}]^{+}: 355.0975$, found: 355.0981 .

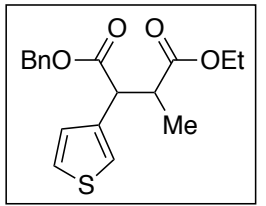




\section{1-Benzyl 4-ethyl 3-methyl-2-(thiophen-3-yl)succinate (2r)}

Colourless oil, 99\% yield. ${ }^{1} \mathrm{H}$ NMR $\left(\mathrm{CDCl}_{3}, 400 \mathrm{MHz}\right): \delta 7.37-7.30(\mathrm{~m}, 3 \mathrm{H}), 7.30-$ $7.25(\mathrm{~m}, 2 \mathrm{H}), 7.23(\mathrm{dd}, J=5.0,3.0 \mathrm{~Hz}, 1 \mathrm{H}), 7.17(\mathrm{dd}, J=3.0,1.4 \mathrm{~Hz}, 1 \mathrm{H}), 7.06(\mathrm{dd}, J=$ $5.0,1.3 \mathrm{~Hz}, 1 \mathrm{H}), 5.16(\mathrm{~d}, J=12.4 \mathrm{~Hz}, 1 \mathrm{H}), 5.10(\mathrm{~d}, J=12.4 \mathrm{~Hz}, 1 \mathrm{H}), 4.02(\mathrm{~d}, J=10.6$ $\mathrm{Hz}, 1 \mathrm{H}), 3.94$ (d, $J=7.1 \mathrm{~Hz}, 2 \mathrm{H}), 3.19$ (dq, $J=10.6,6.9 \mathrm{~Hz}, 1 \mathrm{H}), 1.23(\mathrm{~d}, J=6.9 \mathrm{~Hz}$, $3 \mathrm{H}), 1.02(\mathrm{t}, J=7.1 \mathrm{~Hz}, 3 \mathrm{H}) .{ }^{13} \mathrm{C} \mathrm{NMR}\left(\mathrm{CDCl}_{3}, 100 \mathrm{MHz}\right): \delta 174.2,171.8,136.9,135.7$, $128.7,128.4,128.2,127.7,125.7,123.2,66.9,60.6,50.5,44.0,16.2,14.0$. HRMS-ESI calcd for $[\mathrm{M}+\mathrm{Na}]^{+}: 355.0975$, found: 355.0990 .

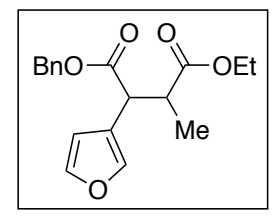

\section{1-Benzyl 4-ethyl 2-(furan-3-yl)-3-methylsuccinate (2s)}

Colourless oil, $99 \%$ yield. ${ }^{1} \mathrm{H}$ NMR $\left(\mathrm{CDCl}_{3}, 400 \mathrm{MHz}\right): \delta 7.40-7.31(\mathrm{~m}, 7 \mathrm{H}), 6.43-6.40$ (m, 1H), $5.19(\mathrm{~d}, J=12.3 \mathrm{~Hz}, 1 \mathrm{H}), 5.15(\mathrm{~d}, J=12.3 \mathrm{~Hz}, 1 \mathrm{H}), 4.08-3.98(\mathrm{~m}, 2 \mathrm{H}), 3.87$ (d, $J=10.0 \mathrm{~Hz}, 1 \mathrm{H}), 3.11(\mathrm{dd}, J=10.0,7.0 \mathrm{~Hz}, 1 \mathrm{H}), 1.24(\mathrm{~d}, J=7.0 \mathrm{~Hz}, 3 \mathrm{H}), 1.13$ (t, $J=$ $7.1 \mathrm{~Hz}, 3 \mathrm{H}) .{ }^{13} \mathrm{C}$ NMR $\left(100 \mathrm{MHz}, \mathrm{CDCl}_{3}\right) \delta 174.1,171.8,143.0,140.6,135.6,128.6$, $128.4,128.3,110.4,66.9,60.6,45.8,43.5,15.9,14.0$. HRMS-ESI calcd for $[\mathrm{M}+\mathrm{Na}]^{+}$: 339.1203, found: 339.1208 .

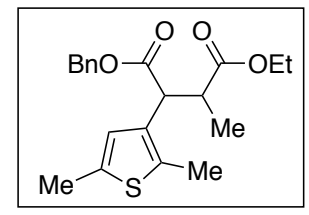

\section{1-Benzyl 4-ethyl 2-(2,5-dimethylthiophen-3-yl)-3-methylsuccinate (2t)}

Yellow oil, 99\% yield. ${ }^{1} \mathrm{H}$ NMR $\left(\mathrm{CDCl}_{3}, 400 \mathrm{MHz}\right): \delta 7.36-7.28(\mathrm{~m}, 3 \mathrm{H}), 7.27-7.23$ $(\mathrm{m}, 2 \mathrm{H}), 6.64-6.61(\mathrm{~m}, 1 \mathrm{H}), 5.15(\mathrm{~d}, J=12.5 \mathrm{~Hz}, 1 \mathrm{H}), 5.08(\mathrm{~d}, J=12.5 \mathrm{~Hz}, 1 \mathrm{H}), 4.01-$ $3.84(\mathrm{~m}, 3 \mathrm{H}), 3.17(\mathrm{dq}, J=10.9,6.9 \mathrm{~Hz}, 1 \mathrm{H}), 2.36-2.33(\mathrm{~m}, 3 \mathrm{H}), 2.33-2.31(\mathrm{~m}, 3 \mathrm{H})$, $1.22(\mathrm{~d}, J=6.8 \mathrm{~Hz}, 3 \mathrm{H}), 1.01(\mathrm{t}, J=7.1 \mathrm{~Hz}, 3 \mathrm{H}) .{ }^{13} \mathrm{C} \mathrm{NMR}\left(\mathrm{CDCl}_{3}, 100 \mathrm{MHz}\right): \delta 174.4$, 172.0, 136.0, 135.6, 133.8, 131.6, 128.6, 128.3, 128.0, 125.0, 66.7, 60.5, 48.4, 43.2, 16.4, 15.3, 14.0, 13.0. HRMS-ESI calcd for $[\mathrm{M}+\mathrm{Na}]^{+}: 383.1288$, found: 383.1300 .

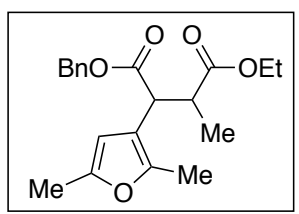

\section{1-Benzyl 4-ethyl 2-(2,5-dimethylfuran-3-yl)-3-methylsuccinate (2u)}

Yellow oil, 93\% yield. ${ }^{1} \mathrm{H}$ NMR $\left(\mathrm{CDCl}_{3}, 400 \mathrm{MHz}\right): \delta 7.38-7.27(\mathrm{~m}, 5 \mathrm{H}), 5.92(\mathrm{~s}, 1 \mathrm{H})$, $5.15(\mathrm{~d}, J=12.4 \mathrm{~Hz}, 1 \mathrm{H}), 5.09(\mathrm{~d}, J=12.4 \mathrm{~Hz}, 1 \mathrm{H}), 4.07-3.90(\mathrm{~m}, 2 \mathrm{H}), 3.67(\mathrm{~d}, J=$ $10.6 \mathrm{~Hz}, 1 \mathrm{H}), 3.07(\mathrm{dq}, J=10.6,6.9 \mathrm{~Hz}, 1 \mathrm{H}), 2.18(\mathrm{~s}, 6 \mathrm{H}), 1.19(\mathrm{~d}, J=6.9 \mathrm{~Hz}, 3 \mathrm{H}), 1.08$ 
(t, $J=7.1 \mathrm{~Hz}, 3 \mathrm{H}) .{ }^{13} \mathrm{C} \mathrm{NMR}\left(\mathrm{CDCl}_{3}, 100 \mathrm{MHz}\right): \delta 174.3,172.2,149.8,147.3,135.9$, $128.7,128.4,128.2,115.5,106.1,66.8,60.5,46.0,43.0,16.2,14.1,13.6,11.6$. HRMSESI calcd for $[\mathrm{M}+\mathrm{Na}]^{+}: 367.1516$, found: 367.1499 .

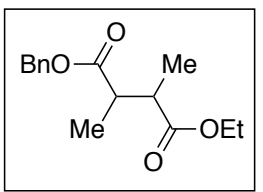

\section{1-Benzyl 4-ethyl 2,3-dimethylsuccinate (2v)}

Colourless oil, $99 \%$ yield. ${ }^{1} \mathrm{H}$ NMR (CDCl $\left.3,400 \mathrm{MHz}\right): \delta 7.40-7.29$ (m, $\left.5 \mathrm{H}\right), 5.14$ (d, $J$ $=12.4 \mathrm{~Hz}, 1 \mathrm{H}), 5.10(\mathrm{~d}, J=12.4 \mathrm{~Hz}, 1 \mathrm{H}), 4.08(\mathrm{qd}, J=7.1,0.9 \mathrm{~Hz}, 2 \mathrm{H}), 2.94-2.74(\mathrm{~m}$, $2 \mathrm{H}), 1.22-1.13(\mathrm{~m}, 9 \mathrm{H}) .{ }^{13} \mathrm{C} \mathrm{NMR}\left(\mathrm{CDCl}_{3}, 100 \mathrm{MHz}\right): \delta 175.3,175.2,136.1,128.7$, $128.3,128.3,66.5,60.7,41.8(2 \mathrm{C}), 14.3,13.8$. HRMS-ESI calcd for $[\mathrm{M}+\mathrm{Na}]^{+}: 287.1254$, found: 287.1262 .

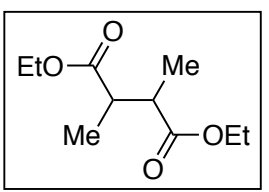

\section{Diethyl 2,3-dimethylsuccinate (2w)}

Colorless oil, ${ }^{1} \mathrm{H}$ NMR $\left(\mathrm{CDCl}_{3}, 400 \mathrm{MHz}\right): \delta 4.12(\mathrm{q}, J=7.1 \mathrm{~Hz}, 4 \mathrm{H}), 2.83-2.73(\mathrm{~m}$, $2 \mathrm{H}), 1.24(\mathrm{t}, J=7.2 \mathrm{~Hz}, 6 \mathrm{H}), 1.17-1.12(\mathrm{~m}, 6 \mathrm{H}) .{ }^{13} \mathrm{C} \mathrm{NMR}\left(\mathrm{CDCl}_{3}, 100 \mathrm{MHz}\right): \delta$ 175.4, $60.6,41.8,14.3,13.8$. HRMS-ESI calcd for $[\mathrm{M}+\mathrm{Na}]^{+}: 225.1097$, found: 225.1099 .

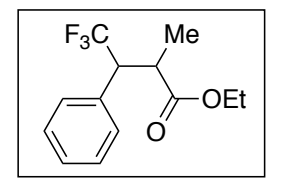

\section{Ethyl 4,4,4-trifluoro-2-methyl-3-phenylbutanoate (6a)}

Colourless oil, 98\% yield, ${ }^{1} \mathrm{H}$ NMR $\left(\mathrm{CDCl}_{3}, 400 \mathrm{MHz}\right) \delta 7.40-7.34(\mathrm{~m}, 3 \mathrm{H}), 7.31-7.26$ (m, 2H), 4.21 (qd, $J=7.1,5.5 \mathrm{~Hz}, 2 \mathrm{H}), 3.71$ (dq, $J=10.5,8.9 \mathrm{~Hz}, 1 \mathrm{H}), 3.07$ (dq, $J=$ $10.4,7.0 \mathrm{~Hz}, 1 \mathrm{H}), 1.30(\mathrm{t}, J=7.1 \mathrm{~Hz}, 3 \mathrm{H}), 0.96(\mathrm{~d}, J=7.0 \mathrm{~Hz}, 3 \mathrm{H}) .{ }^{13} \mathrm{C} \mathrm{NMR}\left(\mathrm{CDCl}_{3}\right.$, $100 \mathrm{MHz}) \delta 174.7,129.7,128.9,128.6,127.9,125.1,61.2,52.6(\mathrm{q}, J=26.3 \mathrm{~Hz}), 39.5(\mathrm{~d}$, $J=1.8 \mathrm{~Hz}), 16.2,14.2 .{ }^{19} \mathrm{~F} \mathrm{NMR}\left(\mathrm{CDCl}_{3}, 376 \mathrm{MHz}\right) \delta-67.7$ (s). HRMS-ESI calcd for [M+Na] $]^{+}: 283.0916$, found: 283.0921 .

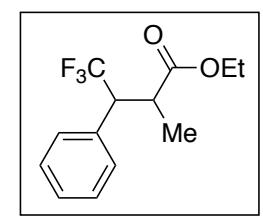

\section{Ethyl 4,4,4-trifluoro-2-methyl-3-phenylbutanoate (6b)}

Colourless oil, $13 \%$ conversion. ${ }^{1} \mathrm{H}$ NMR $\left(\mathrm{CDCl}_{3}, 400 \mathrm{MHz}\right) \delta 7.39-7.30(\mathrm{~m}, 5 \mathrm{H}), 3.88$ $(\mathrm{q}, J=7.1 \mathrm{~Hz}, 2 \mathrm{H}), 3.66-3.53(\mathrm{~m}, 1 \mathrm{H}), 3.27-3.10(\mathrm{~m}, 1 \mathrm{H}), 1.46(\mathrm{dq}, J=6.8,1.7 \mathrm{~Hz}$, $3 \mathrm{H}), 0.96(\mathrm{t}, J=7.1 \mathrm{~Hz}, 3 \mathrm{H}) .{ }^{13} \mathrm{C} \mathrm{NMR}\left(\mathrm{CDCl}_{3}, 100 \mathrm{MHz}\right) \delta 173.7,134.9,134.4,130.9$, 
129.2, 128.6, 128.4, 52.6 (q. $J=26.4$ ), 42.4, 16.5 (t, $J=2.2 \mathrm{~Hz}$ ), 13.9. HRMS-ESI calcd for $[\mathrm{M}+\mathrm{Na}]^{+}: 283.0916$, found: 283.0926 .

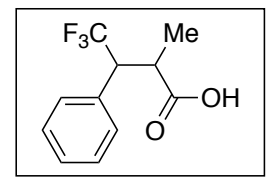

\section{4,4,4-Trifluoro-2-methyl-3-phenylbutanoic acid (6c)}

White crystalline solid, 99\% yield. ${ }^{1} \mathrm{H}$ NMR $\left(\mathrm{CDCl}_{3}, 400 \mathrm{MHz}\right) \delta 7.42-7.36(\mathrm{~m}, 3 \mathrm{H})$, 7.29 (dd, $J=7.2,2.4 \mathrm{~Hz}, 2 \mathrm{H}), 3.85-3.59(\mathrm{~m}, 1 \mathrm{H}), 3.14(\mathrm{dq}, J=10.1,7.1 \mathrm{~Hz}, 1 \mathrm{H}), 1.04$ $(\mathrm{d}, J=7.0 \mathrm{~Hz}, 3 \mathrm{H}) .{ }^{13} \mathrm{C} \mathrm{NMR}\left(\mathrm{CDCl}_{3}, 100 \mathrm{MHz}\right) \delta 180.6,132.7-131.6(\mathrm{~m}), 129.7$, $129.0,128.8,126.5(\mathrm{~d}, J=280.7 \mathrm{~Hz}), 52.4(\mathrm{q}, J=26.4 \mathrm{~Hz}), 39.3(\mathrm{t}, J=1.8 \mathrm{~Hz}), 16.1 .{ }^{19} \mathrm{~F}$ NMR $\left(\mathrm{CDCl}_{3}, 376 \mathrm{MHz}\right) \delta-67.7$ (s). HRMS-ESI calcd for [M+Na] ${ }^{+}: 2255.0609$, found: 255.0635 .

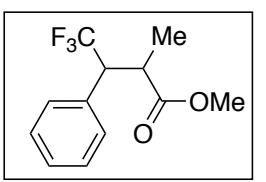

\section{Methyl 4,4,4-trifluoro-2-methyl-3-phenylbutanoate (6d)}

Colourless oil, $88 \%$ yield, ${ }^{1} \mathrm{H}$ NMR $\left(\mathrm{CDCl}_{3}, 400 \mathrm{MHz}\right) \delta 7.43-7.32(\mathrm{~m}, 3 \mathrm{H}), 7.31-7.23$ (m, 2H), $3.76(\mathrm{~s}, 3 \mathrm{H}), 3.70(\mathrm{dt}, J=10.3,8.9 \mathrm{~Hz}, 1 \mathrm{H}), 3.10(\mathrm{dq}, J=10.4,7.1 \mathrm{~Hz}, 1 \mathrm{H})$, $0.96(\mathrm{~d}, J=7.0 \mathrm{~Hz}, 3 \mathrm{H}) .{ }^{13} \mathrm{C}$ NMR $\left(\mathrm{CDCl}_{3}, 100 \mathrm{MHz}\right) \delta 175.2,132.6(\mathrm{~d}, J=1.9 \mathrm{~Hz})$, $129.7-129.6(\mathrm{~m}), 128.9,128.7,126.5(\mathrm{~d}, J=280.7 \mathrm{~Hz}), 52.7$ (q, $J=26.2 \mathrm{~Hz}), 52.4,39.3$ $(\mathrm{d}, J=1.8 \mathrm{~Hz}), 16.1 .{ }^{19} \mathrm{~F}$ NMR $\left(\mathrm{CDCl}_{3}, 376 \mathrm{MHz}\right) \delta-67.8$ (s). HRMS-ESI calcd for $[\mathrm{M}+\mathrm{Na}]^{+}:$269.0765, found: 269.0755 .

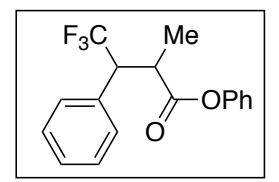

\section{Phenyl 4,4,4-trifluoro-2-methyl-3-phenylbutanoate (6e)}

Colourless oil, $48 \%$ yield, ${ }^{1} \mathrm{H}$ NMR $\left(\mathrm{CDCl}_{3}, 400 \mathrm{MHz}\right) \delta 7.44-7.38(\mathrm{~m}, 5 \mathrm{H}), 7.34(\mathrm{dd}, J$ $=7.3,2.2 \mathrm{~Hz}, 2 \mathrm{H}), 7.26(\mathrm{dt}, J=14.9,1.2 \mathrm{~Hz}, 1 \mathrm{H}), 7.12-7.08(\mathrm{~m}, 2 \mathrm{H}), 3.84(\mathrm{dq}, J=$ $10.5,8.8 \mathrm{~Hz}, 1 \mathrm{H}), 3.34(\mathrm{dq}, J=10.5,7.0 \mathrm{~Hz}, 1 \mathrm{H}), 1.14(\mathrm{~d}, J=7.0 \mathrm{~Hz}, 3 \mathrm{H}) .{ }^{13} \mathrm{C}$ NMR $\left(\mathrm{CDCl}_{3}, 100 \mathrm{MHz}\right) \delta 173.2,150.7,132.5-132.3(\mathrm{~m}), 129.7,129.7,129.7-129.6(\mathrm{~m})$, $129.1,128.8,126.2,121.5,52.7$ (q, $J=26.5 \mathrm{~Hz}), 39.6,16.3 .{ }^{19} \mathrm{~F} \mathrm{NMR}\left(\mathrm{CDCl}_{3}, 376 \mathrm{MHz}\right)$ $\delta$-67.4 (s). HRMS-ESI calcd for [M+Na] $]^{+}: 331.0922$, found: 331.0920 .

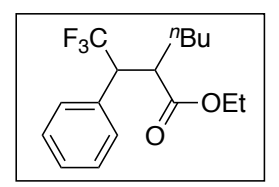

Ethyl 2-(2,2,2-trifluoro-1-phenylethyl) hexanoate (6f)

Colourless oil, $60 \%$ yield, ${ }^{1} \mathrm{H}$ NMR $\left(\mathrm{CDCl}_{3}, 400 \mathrm{MHz}\right) \delta 7.44-7.32(\mathrm{~m}, 3 \mathrm{H}), 7.32-7.20$ (m, 2H), 4.23 (qd, $J=7.1,1.6 \mathrm{~Hz}, 2 \mathrm{H}), 3.64$ (dq, $J=11.3,8.7 \mathrm{~Hz}, 1 \mathrm{H}), 2.97$ (td, $J=11.3$, 
$2.9 \mathrm{~Hz}, 1 \mathrm{H}), 1.31(\mathrm{t}, J=7.2 \mathrm{~Hz}, 4 \mathrm{H}), 1.24-1.02(\mathrm{~m}, 5 \mathrm{H}), 0.80-0.69(\mathrm{~m}, 3 \mathrm{H}) .{ }^{13} \mathrm{C} \mathrm{NMR}$ $\left(\mathrm{CDCl}_{3}, 100 \mathrm{MHz}\right) \delta 174.3,133.1(\mathrm{~d}, J=1.9 \mathrm{~Hz}), 129.5,129.0,128.6,126.4(\mathrm{~d}, J=281.0$ $\mathrm{Hz}), 61.1,52.7(\mathrm{q}, J=26.0 \mathrm{~Hz}), 45.1(\mathrm{~d}, J=1.5 \mathrm{~Hz}), 30.6,28.9,22.2,14.3,13.8 .{ }^{19} \mathrm{~F}$ NMR $\left(\mathrm{CDCl}_{3}, 376 \mathrm{MHz}\right) \delta-67.6$ (s). HRMS-ESI calcd for $[\mathrm{M}+\mathrm{Na}]^{+}: 325.1391$, found: 325.1384 .

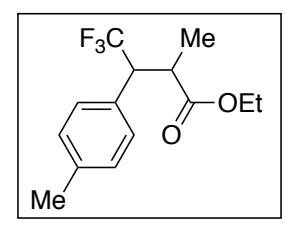

\section{Ethyl 4,4,4-trifluoro-2-methyl-3-p-tolylbutanoate (6g)}

Colourless oil, $68 \%$ yield. ${ }^{1} \mathrm{H}$ NMR $\left(\mathrm{CDCl}_{3}, 400 \mathrm{MHz}\right) \delta 7.22-7.10(\mathrm{~m}, 4 \mathrm{H}), 4.28-4.11$ (m, 2H), 3.67 (dq, $J=10.4,9.0 \mathrm{~Hz}, 1 \mathrm{H}), 3.05$ (dq, $J=10.4,7.1 \mathrm{~Hz}, 1 \mathrm{H}), 2.35(\mathrm{~s}, 3 \mathrm{H})$, $1.30(\mathrm{t}, J=7.1 \mathrm{~Hz}, 3 \mathrm{H}), 0.96(\mathrm{~d}, J=7.1 \mathrm{~Hz}, 3 \mathrm{H}) .{ }^{13} \mathrm{C} \mathrm{NMR}\left(\mathrm{CDCl}_{3}, 100 \mathrm{MHz}\right) \delta 174.8$, $138.4,129.7-129.3(\mathrm{~m}), 129.6,129.5,126.6(\mathrm{~d}, J=280.6 \mathrm{~Hz}), 61.2,52.3$ (q, $J=26.2$ $\mathrm{Hz}), 39.5(\mathrm{q}, J=1.8 \mathrm{~Hz}), 21.2,16.2,14.2 .{ }^{19} \mathrm{~F} \mathrm{NMR}\left(\mathrm{CDCl}_{3}, 376 \mathrm{MHz}\right) \delta-67.8(\mathrm{~s})$. HRMS-ESI calcd for $[\mathrm{M}+\mathrm{Na}]^{+}: 297.1078$, found: 297.1080.

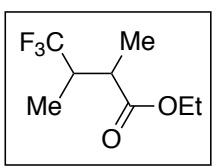

Ethyl 4,4,4-trifluoro-2,3-dimethylbutanoate (6h)(volatile)

Colourless oil (volatile), 99\% yield. ${ }^{1} \mathrm{H}$ NMR $\left(\mathrm{CDCl}_{3}, 400 \mathrm{MHz}\right) \delta 4.16$ (qd, $J=7.1,1.9$ $\mathrm{Hz}, 2 \mathrm{H}), 2.85-2.69(\mathrm{~m}, 2 \mathrm{H}), 1.26(\mathrm{t}, J=7.1 \mathrm{~Hz}, 3 \mathrm{H}), 1.20-1.16(\mathrm{~m}, 3 \mathrm{H}), 1.10(\mathrm{~d}, J=$ $6.9 \mathrm{~Hz}, 3 \mathrm{H}) .{ }^{13} \mathrm{C}$ NMR $\left(\mathrm{CDCl}_{3}, 100 \mathrm{MHz}\right) \delta 174.5,133.0-122.6(\mathrm{~m}), 61.1,39.4(\mathrm{q}, J=$ $26.2 \mathrm{~Hz}), 38.4(\mathrm{q}, J=2.1 \mathrm{~Hz}), 14.2,12.4,9.1(\mathrm{q}, J=2.9 \mathrm{~Hz}) .{ }^{19} \mathrm{~F}$ NMR $\left(\mathrm{CDCl}_{3}, 376\right.$ MHz) $\delta$-71.2 (s).HRMS-ESI calcd for [M+Na] $]^{+}: 221.0765$, found: 221.0743.

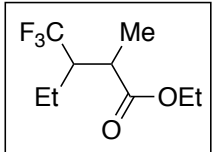

\section{Ethyl 2-methyl-3-(trifluoromethyl) pentanoate (6i)}

Colourless oil (volatile), $99 \%$ yield. ${ }^{1} \mathrm{H}$ NMR $\left(\mathrm{CDCl}_{3}, 400 \mathrm{MHz}\right) \delta 4.15$ (qq, $J=7.3,3.7$ $\mathrm{Hz}, 2 \mathrm{H}), 2.84(\mathrm{qd}, J=7.1,4.4 \mathrm{~Hz}, 1 \mathrm{H}), 2.75-2.57(\mathrm{~m}, 1 \mathrm{H}), 1.65(\mathrm{dp}, J=14.9,7.5 \mathrm{~Hz}$, $1 \mathrm{H}), 1.59-1.48(\mathrm{~m}, 1 \mathrm{H}), 1.26(\mathrm{~d}, J=14.3 \mathrm{~Hz}, 3 \mathrm{H}), 1.18(\mathrm{dd}, J=7.2,1.0 \mathrm{~Hz}, 3 \mathrm{H}), 0.96$ (dd, $J=7.5,1.1 \mathrm{~Hz}, 3 \mathrm{H}) .{ }^{13} \mathrm{C}$ NMR $\left(\mathrm{CDCl}_{3}, 100 \mathrm{MHz}\right) \delta 174.4,128.1(\mathrm{q}, J=281.0 \mathrm{~Hz})$, $61.1,45.7(\mathrm{q}, J=24.6 \mathrm{~Hz}), 37.8(\mathrm{q}, J=2.6 \mathrm{~Hz}), 18.0(\mathrm{q}, J=2.3 \mathrm{~Hz}), 14.2,12.0,11.8 .{ }^{19} \mathrm{~F}$ NMR $\left(\mathrm{CDCl}_{3}, 376 \mathrm{MHz}\right) \delta-67.8$ (s). HRMS-ESI calcd for [M+Na] $]^{+}: 235.0922$, found: 235.0928 .

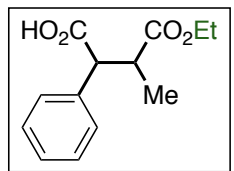

4-Ethoxy-3-methyl-4-oxo-2-phenylbutanoic acid (7) 
White solid, 96\% yield. ${ }^{1} \mathrm{H}$ NMR $\left(\mathrm{CDCl}_{3}, 400 \mathrm{MHz}\right) \delta 10.88$ (bs, $\left.1 \mathrm{H}\right), 7.40-7.20(\mathrm{~m}$, $5 \mathrm{H}), 3.87(\mathrm{q}, J=7.1 \mathrm{~Hz}, 2 \mathrm{H}), 3.80(\mathrm{~d}, J=10.9 \mathrm{~Hz}, 1 \mathrm{H}), 3.23(\mathrm{dq}, J=10.9,6.8 \mathrm{~Hz}, 1 \mathrm{H})$, $1.33(\mathrm{~d}, J=6.8 \mathrm{~Hz}, 3 \mathrm{H}), 0.94(\mathrm{t}, J=7.1 \mathrm{~Hz}, 3 \mathrm{H}) \cdot{ }^{13} \mathrm{C} \mathrm{NMR}\left(\mathrm{CDCl}_{3}, 100 \mathrm{MHz}\right) \delta 178.2$, $174.1,136.2,128.7,128.6,128.1,60.6,55.0,43.5,16.6,13.9$. HRMS-ESI calcd for $[\mathrm{M}+\mathrm{Na}]^{+}:$259.0946, found: 259.0947 .

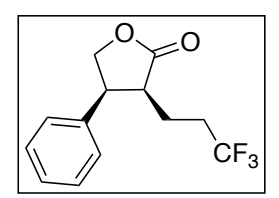

\section{4-Phenyl-3-(3,3,3-trifluoropropyl) dihydrofuran-2(3H)-one (10a)}

White solid, $87 \%$ yield. ${ }^{1} \mathrm{H}$ NMR $\left(\mathrm{CDCl}_{3}, 400 \mathrm{MHz}\right): \delta 7.41-7.32(\mathrm{~m}, 3 \mathrm{H}), 7.22-7.13$ (m, 2H), 4.64 (dd, $J=9.4,6.1 \mathrm{~Hz}, 1 \mathrm{H}), 4.54$ (dd, $J=9.4,1.9 \mathrm{~Hz}, 1 \mathrm{H}), 3.75$ (ddd, $J=8.2$, $6.1,1.9 \mathrm{~Hz}, 1 \mathrm{H}), 2.92(\mathrm{q}, J=7.8 \mathrm{~Hz}, 1 \mathrm{H}), 2.43-2.05(\mathrm{~m}, 2 \mathrm{H}), 1.72$ (dddd, $J=13.9$, $10.0,7.5,6.0 \mathrm{~Hz}, 1 \mathrm{H}), 1.43-1.34(\mathrm{~m}, 1 \mathrm{H}) .{ }^{13} \mathrm{C} \mathrm{NMR}\left(\mathrm{CDCl}_{3}, 100 \mathrm{MHz}\right): \delta 177.7,138.0$, $129.4,128.2,127.5,72.8,44.9,43.2,31.3(\mathrm{q}, J=29.0 \mathrm{~Hz}), 19.3(\mathrm{~d}, J=3.2 \mathrm{~Hz}), 19.2(\mathrm{~d}, J$ $=3.2 \mathrm{~Hz}) .{ }^{19} \mathrm{~F} \mathrm{NMR}\left(\mathrm{CDCl}_{3}, 376 \mathrm{MHz}\right) \delta-66.4(\mathrm{~s})$. HRMS-ESI calcd for $[\mathrm{M}+\mathrm{Na}]^{+}$: 281.0765, found: 281.0761 .

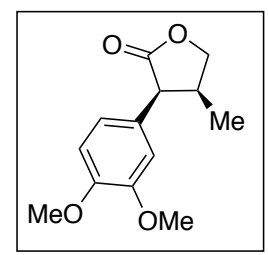

\section{3-(3,4-Dimethoxyphenyl)-4-methyldihydrofuran-2(3H)-one(10b)}

Colourless oil, $56 \%$ yield. ${ }^{1} \mathrm{H}$ NMR $\left(\mathrm{CDCl}_{3}, 400 \mathrm{MHz}\right): \delta 6.86(\mathrm{~d}, J=8.2 \mathrm{~Hz}, 1 \mathrm{H}), 6.77-$ $6.72(\mathrm{~m}, 1 \mathrm{H}), 6.69(\mathrm{~d}, J=2.1 \mathrm{~Hz}, 1 \mathrm{H}), 4.45(\mathrm{dd}, J=9.0,6.3 \mathrm{~Hz}, 1 \mathrm{H}), 4.03(\mathrm{dd}, J=9.0$, $4.6 \mathrm{~Hz}, 1 \mathrm{H}), 3.91$ (d, $J=8.1 \mathrm{~Hz}, 1 \mathrm{H}), 3.88$ (s, $3 \mathrm{H}), 3.87$ (s, 3H), 2.86 (dddd, $J=8.2,7.2$, 6.3, $4.6 \mathrm{~Hz}, 1 \mathrm{H}), 0.81(\mathrm{~d}, J=7.1 \mathrm{~Hz}, 3 \mathrm{H}) \cdot{ }^{13} \mathrm{C} \mathrm{NMR}\left(\mathrm{CDCl}_{3}, 100 \mathrm{MHz}\right): \delta 177.4,149.1$, 148.6, 126.1, 121.7, 112.6, 111.4, 73.0, 56.1, 56.1, 50.2, 36.0, 14.4. HRMS-ESI calcd for $[\mathrm{M}+\mathrm{Na}]^{+}: 259.0946$, found: 259.0948 .

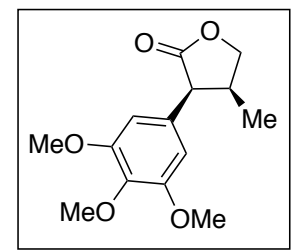

\section{4-Methyl-3-(3,4,5-trimethoxyphenyl) dihydrofuran-2(3H)-one(10c)}

Colourless oil, $69 \%$ yield. ${ }^{1} \mathrm{H}$ NMR $\left(\mathrm{CDCl}_{3}, 400 \mathrm{MHz}\right): \delta 6.40$ (s, $\left.2 \mathrm{H}\right), 4.46$ (dd, $J=9.0$, $6.3 \mathrm{~Hz}, 1 \mathrm{H}), 4.05(\mathrm{dd}, J=9.0,4.5 \mathrm{~Hz}, 1 \mathrm{H}), 3.90(\mathrm{~d}, J=8.2 \mathrm{~Hz}, 1 \mathrm{H}), 3.85(\mathrm{~s}, 6 \mathrm{H}), 3.84$ (s, $3 \mathrm{H}), 2.96-2.79(\mathrm{~m}, 1 \mathrm{H}), 0.83(\mathrm{~d}, J=7.1 \mathrm{~Hz}, 3 \mathrm{H}) .{ }^{13} \mathrm{C} \mathrm{NMR}\left(\mathrm{CDCl}_{3}, 100 \mathrm{MHz}\right)$ : 
$\delta 177.1,153.5,137.6,129.2,106.5,73.0,61.0,56.4,50.7,36.0,14.5$. HRMS-ESI calcd for $[\mathrm{M}+\mathrm{Na}]^{+}: 289.1052$, found: 289.1027 .

Separation data and specific rotation for chiral compounds.

\begin{tabular}{|c|c|c|c|c|c|c|c|c|}
\hline \multirow{2}{*}{ Entry } & \multirow{2}{*}{ Products } & \multirow{2}{*}{$e e(\%)$} & \multicolumn{2}{|c|}{ Retention time } & \multirow{2}{*}{ Separation method } & \multicolumn{3}{|c|}{ Optical rotation } \\
\hline & & & $\mathrm{T}_{\mathrm{R} 1} / \mathrm{min}$ & $\mathrm{T}_{\mathrm{R} 2} / \min$ & & $c$ & $\mathrm{~T}\left({ }^{\circ} \mathrm{C}\right)$ & {$[\alpha]_{D}^{T}$} \\
\hline 1 & & 99 & $\begin{array}{l}14.6 \\
\text { minor }\end{array}$ & $\begin{array}{c}17.2 \\
\text { major }\end{array}$ & $\begin{array}{l}\text { SFC: OJ-H } 5 \% \mathrm{MeOH} \text {, } \\
\text { Flow rate: } 2 \mathrm{~mL} / \mathrm{min}\end{array}$ & 0.28 & 26 & +35.1 \\
\hline 2 & & 95 & $\begin{array}{l}14.8 \\
\text { minor }\end{array}$ & $\begin{array}{l}17.1 \\
\text { major }\end{array}$ & $\begin{array}{l}\text { SFC: OJ-H } 5 \% \mathrm{MeOH} \text {, } \\
\text { Flow rate: } 2 \mathrm{~mL} / \mathrm{min}\end{array}$ & 0.1 & 25 & +24.7 \\
\hline 3 & & 97 & $\begin{array}{l}3.1 \\
\text { minor }\end{array}$ & $\begin{array}{l}3.4 \\
\text { major }\end{array}$ & $\begin{array}{l}\text { SFC: OJ-H } 5 \% \mathrm{MeOH} \text {, } \\
\text { Flow rate: } 2 \mathrm{~mL} / \mathrm{min}\end{array}$ & 0.14 & 25 & +30.5 \\
\hline 4 & & 94 & $\begin{array}{l}3.8 \\
\text { minor }\end{array}$ & $\begin{array}{c}4.1 \\
\text { major }\end{array}$ & $\begin{array}{l}\text { SFC: OJ-H } 2 \% \mathrm{MeOH} \text {, } \\
\text { Flow rate: } 2 \mathrm{~mL} / \mathrm{min}\end{array}$ & 0.22 & 26 & +28.5 \\
\hline 5 & & 90 & $\begin{array}{l}6.7 \\
\text { major }\end{array}$ & $\begin{array}{l}7.3 \\
\text { minor }\end{array}$ & $\begin{array}{l}\text { SFC: } \mathrm{IF} 10 \% \mathrm{MeOH} \text {, } \\
\text { Flow rate: } 2 \mathrm{~mL} / \mathrm{min}\end{array}$ & 0.1 & 25 & +36.0 \\
\hline 6 & & 96 & $\begin{array}{l}3.3 \\
\text { minor }\end{array}$ & $\begin{array}{l}3.5 \\
\text { major }\end{array}$ & $\begin{array}{l}\text { SFC: OJ-H } 5 \% \mathrm{MeOH} \text {, } \\
\text { Flow rate: } 2 \mathrm{~mL} / \mathrm{min}\end{array}$ & 0.26 & 26 & +37.0 \\
\hline 5 & & 97 & $\begin{array}{l}2.9 \\
\text { minor }\end{array}$ & $\begin{array}{c}3.1 \\
\text { major }\end{array}$ & $\begin{array}{l}\text { SFC: OJ-H } 5 \% \mathrm{MeOH}, \\
\text { Flow rate: } 2 \mathrm{~mL} / \mathrm{min}\end{array}$ & 0.20 & 26 & +39.0 \\
\hline 8 & & 94 & $\begin{array}{l}3.7 \\
\text { minor }\end{array}$ & $\begin{array}{l}4.2 \\
\text { major }\end{array}$ & $\begin{array}{l}\text { SFC: OJ-H } 5 \% \mathrm{MeOH} \text {, } \\
\text { Flow rate: } 2 \mathrm{~mL} / \mathrm{min}\end{array}$ & 0.20 & 26 & +26.8 \\
\hline
\end{tabular}




\begin{tabular}{|c|c|c|c|c|c|c|c|c|}
\hline 9 & & 98 & $\begin{array}{l}6.6 \\
\text { minor }\end{array}$ & $\begin{array}{l}7.2 \\
\text { major }\end{array}$ & $\begin{array}{l}\text { SFC: OJ-H } 5 \% \mathrm{MeOH} \text {, } \\
\text { Flow rate: } 2 \mathrm{~mL} / \mathrm{min} \\
\text { (Checked on diol form) }\end{array}$ & 0.10 & 26 & +26.0 \\
\hline 10 & & 97 & $\begin{array}{l}4.0 \\
\text { major }\end{array}$ & $\begin{array}{l}4.3 \\
\text { minor }\end{array}$ & $\begin{array}{l}\text { SFC: OZ-H } 5 \% \mathrm{MeOH}, \\
\text { Flow rate: } 2 \mathrm{~mL} / \mathrm{min}\end{array}$ & 0.20 & 26 & +28.0 \\
\hline 11 & & 91 & $\begin{array}{l}3.5 \\
\text { major }\end{array}$ & $\begin{array}{l}3.9 \\
\text { minor }\end{array}$ & $\begin{array}{l}\text { SFC: OZ-H } 2 \% \mathrm{MeOH}, \\
\text { Flow rate: } 2 \mathrm{~mL} / \mathrm{min}\end{array}$ & 0.14 & 26 & +28.0 \\
\hline 12 & & 97 & $\begin{array}{l}3.0 \\
\text { minor }\end{array}$ & $\begin{array}{l}3.2 \\
\text { major }\end{array}$ & $\begin{array}{l}\text { SFC: OJ-H } 5 \% \mathrm{MeOH} \text {, } \\
\text { Flow rate: } 2 \mathrm{~mL} / \mathrm{min}\end{array}$ & 0.24 & 26 & +40.4 \\
\hline 13 & & 94 & $\begin{array}{l}5.7 \\
\text { major }\end{array}$ & $\begin{array}{c}6.4 \\
\text { minor }\end{array}$ & $\begin{array}{l}\text { SFC: } \mathrm{IB} 15 \% \mathrm{MeOH} \text {, } \\
\text { Flow rate: } 2 \mathrm{~mL} / \mathrm{min}\end{array}$ & 0.1 & 25 & +34.0 \\
\hline 14 & & 96 & $\begin{array}{l}18.7 \\
\text { major }\end{array}$ & $\begin{array}{l}21.7 \\
\text { minor }\end{array}$ & $\begin{array}{l}\text { SFC: } \mathrm{IF} 10 \% \mathrm{MeOH} \text {, } \\
\text { Flow rate: } 2 \mathrm{~mL} / \mathrm{min}\end{array}$ & 0.1 & 25 & +29.0 \\
\hline 15 & & 98 & $\begin{array}{l}6.8 \\
\text { minor }\end{array}$ & $\begin{array}{l}8.1 \\
\text { major }\end{array}$ & $\begin{array}{l}\text { SFC: OJ-H } 5 \% \mathrm{MeOH} \text {, } \\
\text { Flow rate: } 2 \mathrm{~mL} / \mathrm{min}\end{array}$ & 0.14 & 26 & +49.3 \\
\hline 16 & & 62 & $\begin{array}{l}4.6 \\
\text { minor }\end{array}$ & $\begin{array}{l}4.9 \\
\text { major }\end{array}$ & $\begin{array}{l}\text { SFC: OZ-H } 5 \% \mathrm{MeOH}, \\
\text { Flow rate: } 2 \mathrm{~mL} / \mathrm{min}\end{array}$ & 0.18 & 26 & +16.5 \\
\hline 17 & $\mathrm{Br}$ & 89 & $\begin{array}{c}4.1 \\
\text { minor }\end{array}$ & $\begin{array}{l}4.4 \\
\text { major }\end{array}$ & $\begin{array}{l}\text { SFC: OJ-H } 5 \% \mathrm{MeOH}, \\
\text { Flow rate: } 2 \mathrm{~mL} / \mathrm{min}\end{array}$ & 0.22 & 26 & +26.4 \\
\hline 18 & & 75 & $\begin{array}{l}4.2 \\
\text { minor }\end{array}$ & $\begin{array}{l}4.5 \\
\text { major }\end{array}$ & $\begin{array}{l}\text { SFC: OJ-H } 5 \% \mathrm{MeOH} \text {, } \\
\text { Flow rate: } 2 \mathrm{~mL} / \mathrm{min}\end{array}$ & 0.24 & 26 & +10.8 \\
\hline 19 & & 94 & $\begin{array}{l}3.7 \\
\text { minor }\end{array}$ & $\begin{array}{l}3.9 \\
\text { major }\end{array}$ & $\begin{array}{l}\text { SFC: OZ-H } 5 \% \mathrm{MeOH} \text {, } \\
\text { Flow rate: } 2 \mathrm{~mL} / \mathrm{min}\end{array}$ & 0.18 & 26 & +56.5 \\
\hline 20 & & 95 & $\begin{array}{l}6.3 \\
\text { major }\end{array}$ & $\begin{array}{l}7.1 \\
\text { minor }\end{array}$ & $\begin{array}{l}\text { SFC: IA } 5 \% \mathrm{MeOH} \text {, } \\
\text { Flow rate: } 2 \mathrm{~mL} / \mathrm{min}\end{array}$ & 0.20 & 26 & +37.7 \\
\hline 21 & & 81 & $\begin{array}{c}3.1 \\
\text { major }\end{array}$ & $\begin{array}{l}3.5 \\
\text { minor }\end{array}$ & $\begin{array}{l}\text { SFC: AY-H } 5 \% \mathrm{MeOH}, \\
\text { Flow rate: } 2 \mathrm{~mL} / \mathrm{min}\end{array}$ & 0.22 & 26 & +4.9 \\
\hline
\end{tabular}




\begin{tabular}{|c|c|c|c|c|c|c|c|c|}
\hline 22 & MetO- & 84 & $\begin{array}{l}138.1 \\
\text { major }\end{array}$ & $\begin{array}{l}141.5 \\
\text { minor }\end{array}$ & $\begin{array}{c}\text { GC-MS: Hydrodex } \beta \text { - } \\
\text { 3P, } 80^{\circ} \mathrm{C} \text { iso }\end{array}$ & 0.10 & 23 & +5.0 \\
\hline 23 & & 90 & $\begin{array}{c}6.2 \\
\text { major }\end{array}$ & $\begin{array}{l}7.0 \\
\text { minor }\end{array}$ & $\begin{array}{c}\text { SFC: AD-H } 5 \% \mathrm{MeOH} \text {, } \\
\text { Flow rate: } 2 \mathrm{~mL} / \mathrm{min} \\
\text { (Checked on alcohol } \\
\text { form) }\end{array}$ & 0.10 & 24 & +51.0 \\
\hline 24 & & 94 & $\begin{array}{l}29.0 \\
\text { minor }\end{array}$ & $\begin{array}{l}31.0 \\
\text { major }\end{array}$ & $\begin{array}{c}\text { GC-MS: Hydrodex } \beta \text { - } \\
\text { 3P, } 100{ }^{\circ} \mathrm{C} \text { iso }\end{array}$ & 0.10 & 24 & -26.0 \\
\hline 25 & & 90 & $\begin{array}{l}139.0 \\
\text { major }\end{array}$ & $\begin{array}{l}142.0 \\
\text { minor }\end{array}$ & $\begin{array}{l}\text { GC-MS: Hydrodex } \beta \text { - } \\
3 \mathrm{P}, 120^{\circ} \mathrm{C} \text { iso }\end{array}$ & 0.10 & 23 & -15.0 \\
\hline 26 & & 95 & $\begin{array}{l}64.7 \\
\text { minor }\end{array}$ & $\begin{array}{l}65.4 \\
\text { major }\end{array}$ & $\begin{array}{c}\text { GC-MS: Hydrodex } \beta \text { - } \\
3 \mathrm{P}, 80^{\circ} \mathrm{C} \text { iso }\end{array}$ & 0.10 & 22 & +85 \\
\hline 27 & & 97 & $\begin{array}{l}171.0 \\
\text { minor }\end{array}$ & $\begin{array}{l}179.0 \\
\text { major }\end{array}$ & $\begin{array}{l}\text { GC-MS: Hydrodex } \beta \text { - } \\
\text { 6TBDM, } 70{ }^{\circ} \mathrm{C} \text { iso }\end{array}$ & 0.10 & 24 & -41.0 \\
\hline 28 & & 84 & $\begin{array}{l}106.0 \\
\text { minor }\end{array}$ & $\begin{array}{l}111.0 \\
\text { major }\end{array}$ & $\begin{array}{c}\text { GC-MS: Hydrodex } \beta \text { - } \\
\text { 6TBDM, } 70{ }^{\circ} \mathrm{C} \text { iso }\end{array}$ & 0.10 & 24 & -17.0 \\
\hline 29 & & 96 & $\begin{array}{l}5.8 \\
\text { major }\end{array}$ & $\begin{array}{l}7.9 \\
\text { minor }\end{array}$ & $\begin{array}{l}\text { SFC: } \mathrm{OJH} 2 \% \mathrm{MeOH} \\
\text { Flow rate: } 2 \mathrm{~mL} / \mathrm{min}\end{array}$ & 0.10 & 25 & $\begin{array}{c}- \\
143.0\end{array}$ \\
\hline 30 & & 93 & $\begin{array}{l}106.7 \\
\text { minor }\end{array}$ & $\begin{array}{l}110.7 \\
\text { major }\end{array}$ & $\begin{array}{c}\text { GC-MS: Hydrodex } \beta \text { - } \\
\text { 6TBDM, } 70^{\circ} \mathrm{C} \text { iso }\end{array}$ & 0.10 & 25 & -46.0 \\
\hline 31 & & 91 & $\begin{array}{l}5.8 \\
\text { major }\end{array}$ & $\begin{array}{l}7.9 \\
\text { minor }\end{array}$ & $\begin{array}{l}\text { SFC: OJH } 2 \% \mathrm{MeOH} \\
\text { Flow rate: } 2 \mathrm{~mL} / \mathrm{min}\end{array}$ & 0.10 & 25 & -53.0 \\
\hline 32 & & $>99$ & $\begin{array}{c}4.1 \\
\text { major }\end{array}$ & $\begin{array}{l}5.2 \\
\text { minor }\end{array}$ & $\begin{array}{l}\text { SFC: OJH5\% MeOH, } \\
\text { Flow rate: } 2 \mathrm{~mL} / \mathrm{min}\end{array}$ & 0.10 & 25 & -58.0 \\
\hline 33 & $\mathrm{CO}_{2} \mathrm{Et}$ & 97 & $\begin{array}{l}35.7 \\
\text { major }\end{array}$ & $\begin{array}{l}36.5 \\
\text { minor }\end{array}$ & $\begin{array}{c}\text { GC-MS: Hydrodex } \beta \text { - } \\
\mathrm{DM}, 90{ }^{\circ} \mathrm{C} \text { iso }\end{array}$ & 0.10 & 25 & -18.0 \\
\hline 34 & $\mathrm{~F}_{3} \mathrm{C}$ & 88 & $\begin{array}{l}19.9 \\
\text { major }\end{array}$ & $\begin{array}{l}22.4 \\
\text { minor }\end{array}$ & $\begin{array}{c}\text { GC-MS: Hydrodex } \beta \text { - } \\
\mathrm{DM}, 30{ }^{\circ} \mathrm{C} \text { iso }\end{array}$ & 0.10 & 25 & -06.0 \\
\hline 35 & $\underset{\mathrm{Et}}{\mathrm{Ft}_{3} \mathrm{C}}$ & 82 & $\begin{array}{l}41.6 \\
\text { major }\end{array}$ & $\begin{array}{l}48.5 \\
\text { minor }\end{array}$ & $\begin{array}{c}\text { GC-MS: Hydrodex } \beta \text { - } \\
\text { DM, } 30^{\circ} \mathrm{C} \text { iso }\end{array}$ & 0.10 & 25 & -44.0 \\
\hline
\end{tabular}




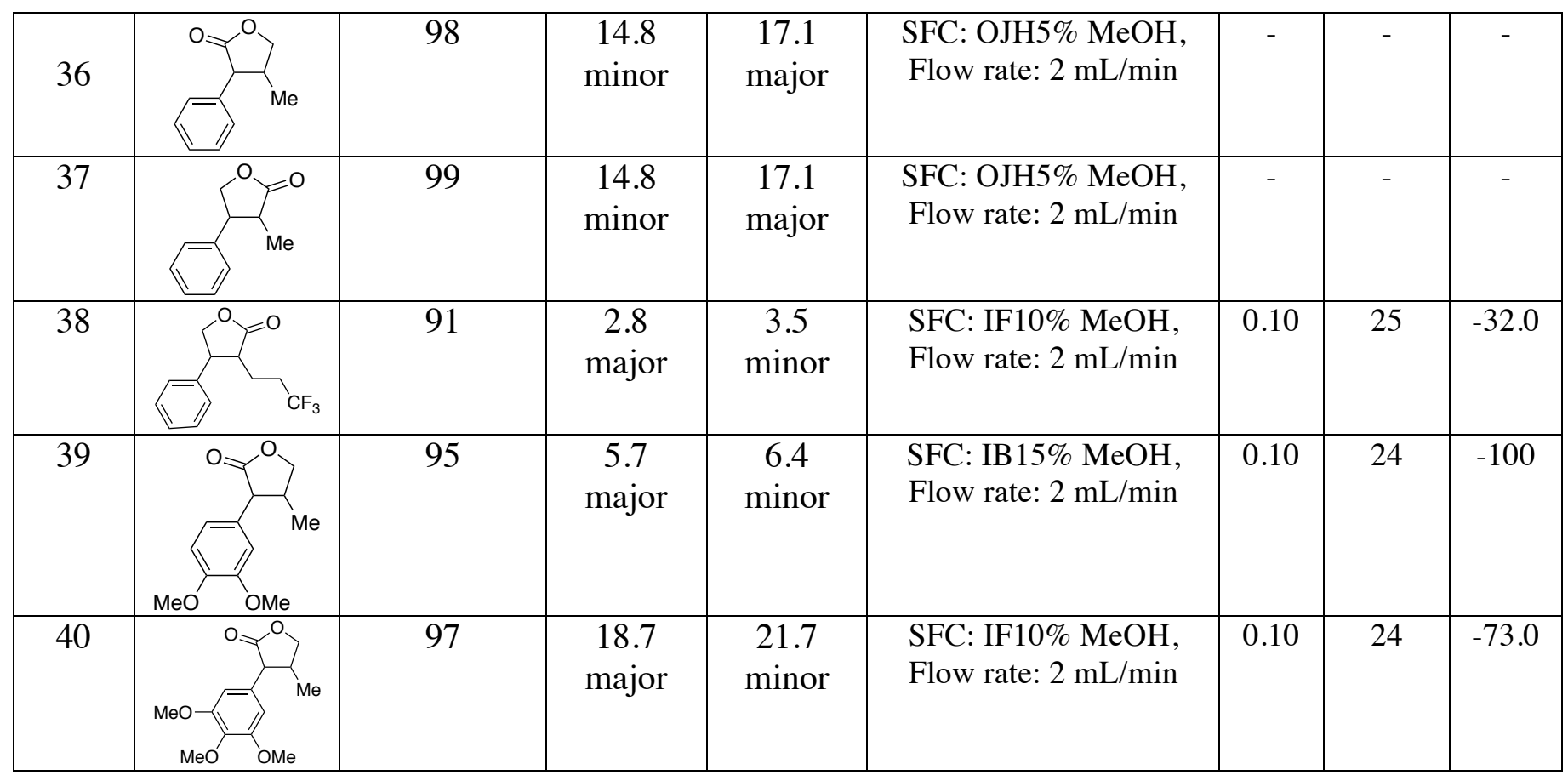

${ }^{\mathrm{a}} \mathrm{All}$ specific rotations were measured for $\mathrm{CHCl}_{3}$ solution

\section{Procedure for hydrogenolysis}
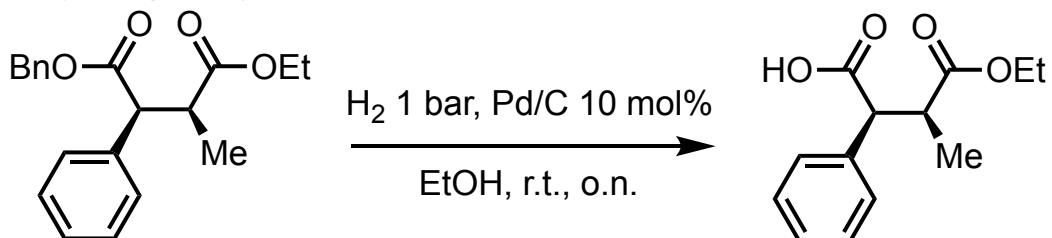

A well-dried glass vial was charged with 1-benzyl 4-ethyl (2S,3S)-3-methyl-2phenylsuccinate $\mathbf{2 b}(0.1 \mathrm{mmol})$ and $\mathrm{Pd} / \mathrm{C}(10 \mathrm{~mol} \%)$. Freshly dried EtOH was added and the flask was placed in a low-pressure hydrogenation apparatus. The reactor was purged three times with $\mathrm{Ar}$ and three times with $\mathrm{H}_{2}$, and then filled with 1 bar $\mathrm{H}_{2}$ gas. The reaction was stirred overnight at room temperature before releasing the $\mathrm{H}_{2}$ pressure. The reaction mixture was filtered through a short pad of celite and the solvent was removed under reduced pressure. The crude product was subjected to short flash chromatographyusing n-pentane, $\mathrm{Et}_{2} \mathrm{O}$ mixture to afford the corresponding $(2 S, 3 S)$-4-ethoxy-3-methyl-4oxo-2-phenylbutanoic acid 7.

Procedure for the selective reduction of $-\mathrm{COOH}$ followed by cyclization for $(3 S, 4 S)$ 3-methyl-4-phenyldihydrofuran-2(3H)-one

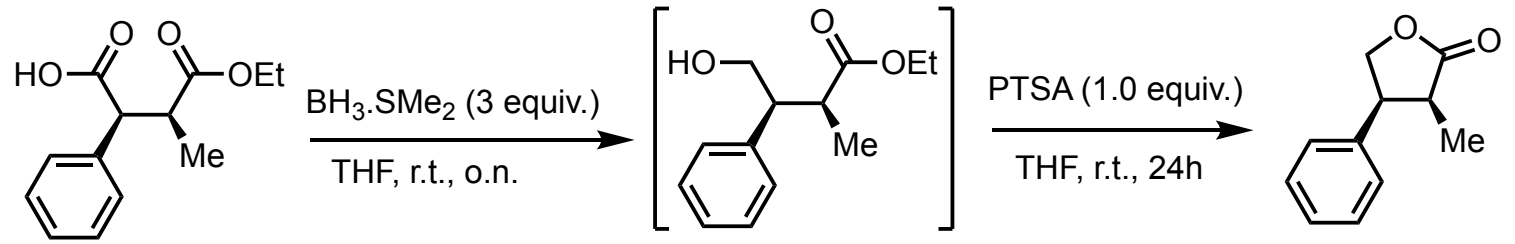


In a $10 \mathrm{~mL}$ one-necked round-bottomed flask, under an argon atmosphere $(2 S, 3 S)-4$ ethoxy-3-methyl-4-oxo-2-phenylbutanoic acid $7(23.6 \mathrm{mg}, 0.1 \mathrm{mmol})$ was dissolved in dry THF $(2 \mathrm{~mL})$ and cooled to $0{ }^{\circ} \mathrm{C}$. $\mathrm{BH}_{3} . \mathrm{SMe}_{2}$ complex (3 equiv.) was added dropwise at this temperature. After addition, the resulting mixture was stirred overnight at room temperature. After completion of the reaction, monitored by TLC, the reaction was quenched with $\mathrm{MeOH}(2 \mathrm{~mL})$ and the volatiles were evaporated in vacuo. The last operation was repeated twice to give the corresponding monoester.

The monoester was dissolved in $2 \mathrm{~mL}$ of THF and PTSA (1 equiv.) was carefully added. The reaction mixture was further stirred at room temperature for another 24 hour. After completion of the reaction, the solvent was evaporated in vacuo and quenched with $\mathrm{H}_{2} \mathrm{O}$ $(2 \mathrm{~mL})$ followed by extraction in $\mathrm{Et}_{2} \mathrm{O}(3 \times 5 \mathrm{~mL})$. The combined organic layers were dried over $\mathrm{Na}_{2} \mathrm{SO}_{4}$, filtered, and concentrated under reduced pressure. The crude product was subjected to flash chromatography using n-pentane, $\mathrm{Et}_{2} \mathrm{O}$ mixture to afford the corresponding $(3 S, 4 S)$-3-methyl-4-phenyldihydrofuran-2(3H)-one 8.

\section{Procedure for the selective reduction of -COOEt followed by cyclization for $(3 S, 4 S)$ - 4-methyl-3-phenyldihydrofuran-2(3H)-one}

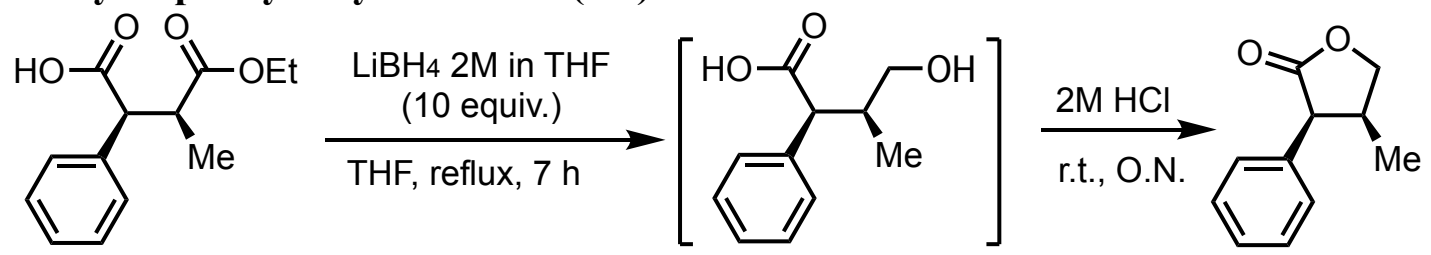

In an oven-dried $10 \mathrm{~mL}$ round-bottomed flask, under argon atmosphere $(2 S, 3 S)$-4-ethoxy3-methyl-4-oxo-2-phenylbutanoic acid7 $(23.6 \mathrm{mg}, 0.1 \mathrm{mmol})$ was dissolved in dry THF $(2 \mathrm{~mL})$ and $\mathrm{LiBH}_{4}$ solution $2 \mathrm{M}$ in THF (10 equiv.) was added dropwise. After addition, the resulting mixture was refluxed for 7 hour. After completion of the reaction, monitored by TLC, the reaction was quenched by $2 \mathrm{M} \mathrm{HCl}(1 \mathrm{~mL})$ under hot conditions. The resulting reaction mixture was concentrated in vacuo and extracted in $\mathrm{Et}_{2} \mathrm{O}(3 \times 5 \mathrm{~mL})$. The combined organic layers were dried over $\mathrm{Na}_{2} \mathrm{SO}_{4}$, filtered and concentrated under reduced pressure. The crude product was purified by flash chromatography-using npentane, $\mathrm{Et}_{2} \mathrm{O}$ mixture to give $(3 S, 4 S)$-4-methyl-3-phenyldihydrofuran-2(3H)-one 9.

\section{General procedure for the preparation of bicyclic ligands}

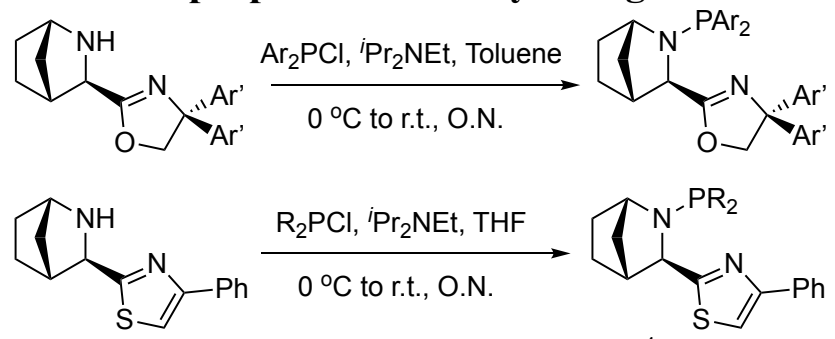

Amine was prepared according to the literature procedure. ${ }^{4}$

Amine $(1 \mathrm{mmol})$ was co-evaporated with dry toluene $(3 \times 20 \mathrm{~mL})$ and dissolved in dry toluene or THF $(6 \mathrm{~mL})$ under $\mathrm{N}_{2}$. Freshly distilled di-iso-propylethylamine $(3 \mathrm{mmol})$ was added and the solution was cooled to $0{ }^{\circ} \mathrm{C}$ in an ice-bath. Freshly distilled (prepared) suitable phosphine chloride $(1.2 \mathrm{mmol})$ was dissolved in toluene $(2 \mathrm{~mL})$ and added dropwise to the reaction mixture and stirred at room temperature for 24 hours. After 
completion of the reaction, monitored by TLC, it was quenched with saturated $\mathrm{NaHCO}_{3}$ solution the reaction mixture was extracted in $\mathrm{CH}_{2} \mathrm{Cl}_{2}(3 \times 10 \mathrm{~mL})$. The combined organic layers were dried over $\mathrm{Na}_{2} \mathrm{SO}_{4}$, filtered and concentrated under reduced pressure. The oily residual was quickly passed through a small pad of silica and used to make the Ir-complex without further characterization.

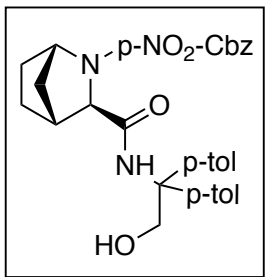

${ }^{1} \mathrm{H} \mathrm{NMR}\left(\mathrm{CDCl}_{3}, 400 \mathrm{MHz}\right) \delta 8.16-8.08(\mathrm{~m}, 2 \mathrm{H}), 7.52-7.37(\mathrm{~m}, 2 \mathrm{H}), 7.16-7.04(\mathrm{~m}$, $6 \mathrm{H}), 7.06-6.85(\mathrm{~m}, 2 \mathrm{H}), 5.29-5.13(\mathrm{~m}, 2 \mathrm{H}), 4.28(\mathrm{~s}, 3 \mathrm{H}), 3.99-3.84(\mathrm{~m}, 1 \mathrm{H}), 2.97(\mathrm{~s}$, $1 \mathrm{H}), 2.33(\mathrm{~s}, 3 \mathrm{H}), 1.98-1.72(\mathrm{~m}, 2 \mathrm{H}), 1.74-1.61(\mathrm{~m}, 2 \mathrm{H}), 1.57-1.37(\mathrm{~m}, 2 \mathrm{H}) .{ }^{13} \mathrm{C}$ NMR $\left(\mathrm{CDCl}_{3}, 100 \mathrm{MHz}\right) \delta 171.3,147.8,139.4,137.4,129.4,129.3,128.3,127.1,126.1$, $124.0,69.9,68.7,67.8,66.2,58.6,40.6,36.6,30.4,26.8,21.1,21.1$. HRMS-ESI; $\mathrm{m} / \mathrm{z}$ $\left[\mathrm{M}^{+}+\mathrm{Na}\right]$ Calcd. for $\mathrm{C}_{31} \mathrm{H}_{33} \mathrm{~N}_{3} \mathrm{O}_{6}=566.2262$. Found: 566.2262. $[\alpha]^{25}{ }_{\mathrm{D}}=+79.00(c=0.1 \mathrm{in}$ $\left.\mathrm{CHCl}_{3}\right)$.

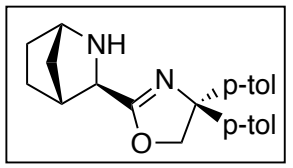

${ }^{1} \mathrm{H}$ NMR $\left(\mathrm{CDCl}_{3}, 400 \mathrm{MHz}\right) \delta 7.18(\mathrm{dd}, J=8.3,3.5 \mathrm{~Hz}, 4 \mathrm{H}), 7.11(\mathrm{dd}, J=8.1,6.4 \mathrm{~Hz}$, $4 \mathrm{H}), 4.79(\mathrm{~s}, 2 \mathrm{H}), 3.59-3.53(\mathrm{~m}, 1 \mathrm{H}), 3.54-3.49(\mathrm{~m}, 1 \mathrm{H}), 2.63(\mathrm{~d}, J=3.2 \mathrm{~Hz}, 1 \mathrm{H}), 2.32$ $(\mathrm{s}, 3 \mathrm{H}), 2.31(\mathrm{~s}, 3 \mathrm{H}), 1.70-1.55(\mathrm{~m}, 3 \mathrm{H}), 1.55-1.44(\mathrm{~m}, 2 \mathrm{H}), 1.30-1.21(\mathrm{~m}, 1 \mathrm{H}) .{ }^{13} \mathrm{C}$ NMR $\left(\mathrm{CDCl}_{3}, 100 \mathrm{MHz}\right) \delta 167.9,143.3,143.1,136.6,129.0,129.0,126.4,126.4,81.0$, 78.3, 59.4, 56.2, 41.4, 35.8, 31.2, 28.4, 21.0. HRMS-ESI; $\mathrm{m} / \mathrm{z}\left[\mathrm{M}^{+}+\mathrm{H}\right]$ Calcd. for $\mathrm{C}_{23} \mathrm{H}_{26} \mathrm{~N}_{2} \mathrm{O}=347.2118$. Found: 347.2112 . $[\alpha]^{25}=+31.00\left(c=0.1\right.$ in $\left.\mathrm{CHCl}_{3}\right)$.

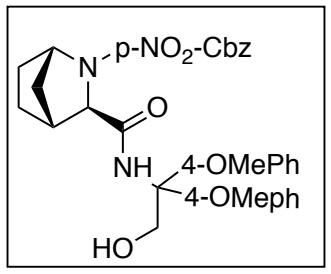

${ }^{1} \mathrm{H}$ NMR $\left(\mathrm{CDCl}_{3}, 400 \mathrm{MHz}\right) \delta 8.10(\mathrm{dd}, J=8.7,2.3 \mathrm{~Hz}, 2 \mathrm{H}), 7.41(\mathrm{~d}, J=8.2 \mathrm{~Hz}, 2 \mathrm{H})$, $7.17-6.93(\mathrm{~m}, 4 \mathrm{H}), 6.80(\mathrm{~d}, J=8.3 \mathrm{~Hz}, 4 \mathrm{H}), 5.41-5.06(\mathrm{~m}, 4 \mathrm{H}), 4.29(\mathrm{~s}, 1 \mathrm{H}), 4.19(\mathrm{~d}, J$ $=7.2 \mathrm{~Hz}, 2 \mathrm{H}), 3.91(\mathrm{~d}, J=16.8 \mathrm{~Hz}, 1 \mathrm{H}), 3.76(\mathrm{~s}, 6 \mathrm{H}), 2.94(\mathrm{~s}, 1 \mathrm{H}), 1.95-1.74(\mathrm{~m}, 2 \mathrm{H})$, $1.74-1.61(\mathrm{~m}, 2 \mathrm{H}), 1.48(\mathrm{dt}, J=12.7,7.0 \mathrm{~Hz}, 1 \mathrm{H}), 1.45-1.34(\mathrm{~m}, 1 \mathrm{H}) .{ }^{13} \mathrm{C} \mathrm{NMR}$ $\left(\mathrm{CDCl}_{3}, 100 \mathrm{MHz}\right) \delta 171.1,158.8,156.4,147.6,143.5,134.4,128.3,128.1,123.8,113.7$, 69.9, 68.0, 67.6, 66.0, 58.4, 55.2, 55.2, 40.5, 36.4, 30.2, 26.7. HRMS-ESI; $m / z\left[\mathrm{M}^{+}+\mathrm{Na}\right]$ Calcd. for $\mathrm{C}_{31} \mathrm{H}_{33} \mathrm{~N}_{3} \mathrm{O}_{8}=598.2160$. Found: 598.2157. $[\alpha]^{25}=+72.00\left(c=0.1\right.$ in $\left.\mathrm{CHCl}_{3}\right)$. 


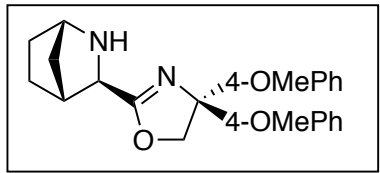

${ }^{1} \mathrm{H}$ NMR $\left(\mathrm{CDCl}_{3}, 400 \mathrm{MHz}\right) \delta 7.24-7.10(\mathrm{~m}, 4 \mathrm{H}), 6.86-6.74(\mathrm{~m}, 4 \mathrm{H}), 4.72(\mathrm{~s}, 2 \mathrm{H})$, $3.78-3.69(\mathrm{~m}, 3 \mathrm{H}), 3.51(\mathrm{~s}, 1 \mathrm{H}), 3.47(\mathrm{~d}, J=1.4 \mathrm{~Hz}, 1 \mathrm{H}), 2.65-2.59(\mathrm{~m}, 1 \mathrm{H}), 2.45(\mathrm{~s}$, $1 \mathrm{H}), 1.68-1.54(\mathrm{~m}, 2 \mathrm{H}), 1.50-1.42(\mathrm{~m}, 2 \mathrm{H}), 1.30-1.17(\mathrm{~m}, 2 \mathrm{H}) .{ }^{13} \mathrm{C} \mathrm{NMR}\left(\mathrm{CDCl}_{3}\right.$, $100 \mathrm{MHz}) \delta 167.9,158.4,138.5,138.3,127.6,113.6,113.6,81.0,77.8,59.3,56.1,55.1$, 41.3, 35.8, 31.3, 28.4, 22.3, 14.0. HRMS-ESI; $m / z\left[\mathrm{M}^{+}+\mathrm{H}\right]$ Calcd. for $\mathrm{C}_{23} \mathrm{H}_{26} \mathrm{~N}_{2} \mathrm{O}_{2}=$ 379.2016. Found: 379.2019 . $[\alpha]^{25}=-11\left(c=0.1\right.$ in $\left.\mathrm{CHCl}_{3}\right)$.

\section{General procedure for the preparation of iridium complexes}

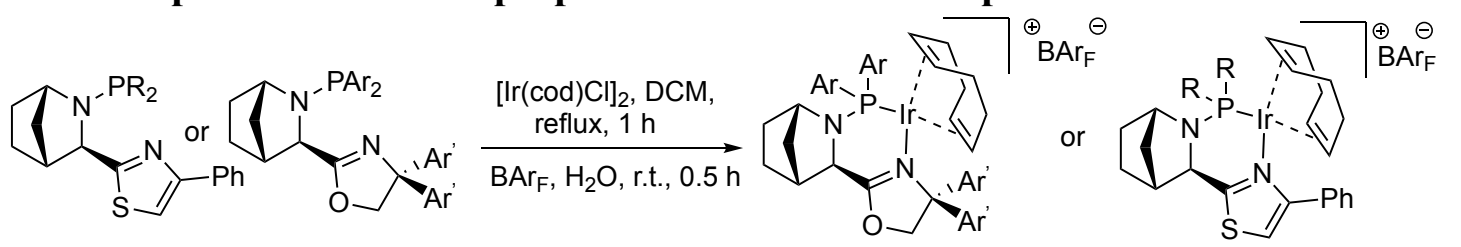

The synthesized oxazoline ligand was dissolved in $\mathrm{CH}_{2} \mathrm{Cl}_{2}(20 \mathrm{~mL})$ and $[\mathrm{Ir}(\mathrm{COD}) \mathrm{Cl}]_{2}$ $(0.25 \mathrm{mmol})$ was added. The atmosphere in the flask was evacuated and replenished three times with $\mathrm{N}_{2}$. The mixture was heated to reflux for 1 hour. After the solution was cooled to room temperature, distilled $\mathrm{H}_{2} \mathrm{O}(20 \mathrm{~mL})$ was added. Under vigorous stirring, $\mathrm{NaBArF} \cdot \mathrm{xH}_{2} \mathrm{O}(0.6 \mathrm{mmol})$ was added to the biphasic solution in one portion. The mixture was stirred vigorously for 30 minutes and extracted with $\mathrm{CH}_{2} \mathrm{Cl}_{2}(3 \times 10 \mathrm{~mL})$. Combined organic phase was dried over $\mathrm{Na}_{2} \mathrm{SO}_{4}$. After concentration in vacuum, the residue was purified on silica gel with $\mathrm{CH}_{2} \mathrm{Cl}_{2}$ : pentane $(1: 1)$ as the eluent to afford the iridium complex as an orange solid.

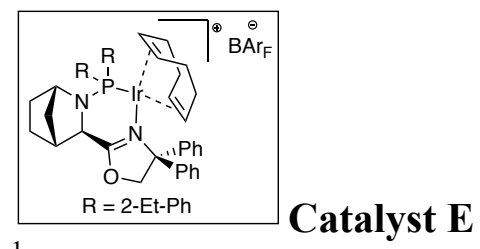

${ }^{1} \mathrm{H}$ NMR $\left(\mathrm{CDCl}_{3}, 400 \mathrm{MHz}\right) \delta 7.76-7.64(\mathrm{~m}, 8 \mathrm{H}), 7.60(\mathrm{t}, J=6.5 \mathrm{~Hz}, 1 \mathrm{H}), 7.55-7.46$ $(\mathrm{m}, 7 \mathrm{H}), 7.38-7.16(\mathrm{~m}, 9 \mathrm{H}), 7.09-6.83(\mathrm{~m}, 5 \mathrm{H}), 5.11-4.97(\mathrm{~m}, 2 \mathrm{H}), 4.92(\mathrm{~d}, J=9.0$ $\mathrm{Hz}, 1 \mathrm{H}), 4.58-4.48(\mathrm{~m}, 1 \mathrm{H}), 4.48-4.35(\mathrm{~m}, 1 \mathrm{H}), 4.35-4.23(\mathrm{~m}, 1 \mathrm{H}), 3.54-3.42(\mathrm{~m}$, $1 \mathrm{H}), 3.43-3.19(\mathrm{~m}, 2 \mathrm{H}), 3.11-3.03(\mathrm{~m}, 1 \mathrm{H}), 2.40-2.16(\mathrm{~m}, 3 \mathrm{H}), 2.15-1.77(\mathrm{~m}, 4 \mathrm{H})$, $1.77-1.37(\mathrm{~m}, 7 \mathrm{H}), 1.37-0.79(\mathrm{~m}, 5 \mathrm{H}), 1.19-0.70(\mathrm{~m}, 3 \mathrm{H}), 0.64-0.42(\mathrm{~m}, 1 \mathrm{H}) .{ }^{13} \mathrm{C}$ NMR $\left(\mathrm{CDCl}_{3}, 100 \mathrm{MHz}\right) \delta 173.9,161.8(\mathrm{dd}, J=99.9,49.8 \mathrm{~Hz}), 142.3,137.8,135.5-$ $134.6(\mathrm{~m}), 133.0-132.5(\mathrm{~m}), 129.9,129.7-129.0(\mathrm{~m}), 129.1-128.4(\mathrm{~m}), 128.3,126.4$ $(\mathrm{d}, J=14.0 \mathrm{~Hz}), 126.1,123.3,120.6,117.6,9.15,87.4,82.2,68.5,63.5$ (d, $J=10.8 \mathrm{~Hz})$, 60.8, 58.2, 39.7, 36.0, 34.4, $29.0(\mathrm{~d}, J=30.6 \mathrm{~Hz}), 27.1,15.3,13.6 .{ }^{31} \mathrm{P} \mathrm{NMR}\left(\mathrm{CDCl}_{3}, 121\right.$ $\mathrm{MHz}) \delta$ 48.7. HRMS-ESI calcd for $\left[\mathrm{M}-\mathrm{BAr}_{\mathrm{F}}\right]^{+}: 859.3366$, found: $859.3357 .[\alpha]_{\mathrm{D}}^{25}=$ $+10.00\left(c=0.1\right.$ in $\left.\mathrm{CHCl}_{3}\right)$. 


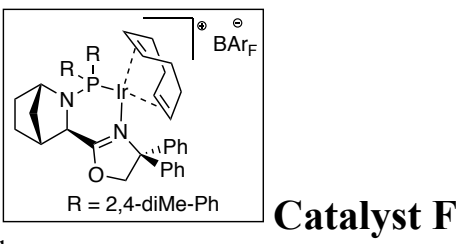

${ }^{1} \mathrm{H}$ NMR $\left(\mathrm{CDCl}_{3}, 400 \mathrm{MHz}\right) \delta 7.76-7.68(\mathrm{~m}, 8 \mathrm{H}), 7.57-7.50(\mathrm{~m}, 6 \mathrm{H}), 7.36-7.28(\mathrm{~m}$, $3 \mathrm{H}), 7.26-7.16(\mathrm{~m}, 2 \mathrm{H}), 7.15-7.00(\mathrm{~m}, 4 \mathrm{H}), 7.00-6.92(\mathrm{~m}, 3 \mathrm{H}), 6.82-6.67(\mathrm{~m}, 2 \mathrm{H})$, $5.09-4.97(\mathrm{~m}, 2 \mathrm{H}), 4.93(\mathrm{~d}, J=8.9 \mathrm{~Hz}, 1 \mathrm{H}), 4.60(\mathrm{~s}, 1 \mathrm{H}), 4.40(\mathrm{~d}, J=2.3 \mathrm{~Hz}, 1 \mathrm{H}), 3.41$ $-3.38(\mathrm{~m}, 2 \mathrm{H}), 3.30-3.26(\mathrm{~m}, 1 \mathrm{H}), 3.18(\mathrm{~s}, 3 \mathrm{H}), 3.11-3.00(\mathrm{~m}, 1 \mathrm{H}), 2.36(\mathrm{~s}, 4 \mathrm{H}), 2.20$ $(\mathrm{s}, 3 \mathrm{H}), 2.16(\mathrm{~s}, 3 \mathrm{H}), 2.13-2.05(\mathrm{~m}, 1 \mathrm{H}), 2.00(\mathrm{~d}, J=10.0 \mathrm{~Hz}, 1 \mathrm{H}), 1.88-1.65(\mathrm{~m}, 5 \mathrm{H})$, $1.61-1.52(\mathrm{~m}, 1 \mathrm{H}), 1.51-1.43(\mathrm{~m}, 1 \mathrm{H}), 1.41-1.32(\mathrm{~m}, 2 \mathrm{H}), 1.19-1.12(\mathrm{~m}, 1 \mathrm{H}), 1.07$ $1,02(\mathrm{~m}, 1 \mathrm{H}), 0.84-0.79(\mathrm{~m}, 1 \mathrm{H}) .{ }^{13} \mathrm{C} \mathrm{NMR}\left(\mathrm{CDCl}_{3}, 100 \mathrm{MHz}\right) \delta 173.2,161.8(\mathrm{dd}, J=$ 99.7, $49.8 \mathrm{~Hz}), 142.8(\mathrm{~d}, J=22.5 \mathrm{~Hz}), 141.5(\mathrm{~d}, J=8.1 \mathrm{~Hz}), 137.9,135.6-133.6(\mathrm{~m})$, $134.1(\mathrm{~d}, J=9.1 \mathrm{~Hz}), 133.2(\mathrm{~d}, J=8.8 \mathrm{~Hz}), 132.9,129.9,129.6-129.0(\mathrm{~m}), 129.0-$ $128.7(\mathrm{~m}), 128.5(\mathrm{~d}, J=11.5 \mathrm{~Hz}), 128.1,127.6,127.3$ (d, $J=9.4 \mathrm{~Hz}), 126.3,126.1$, 123.6, 120.6, 118.0 - 117.1 (m), 86.8, 77.5, 68.9, 62.3, 58.1, 40.6, 39.6, 36.1, 34.0, 29.8, 29.1, $25.2(\mathrm{~d}, J=30.5 \mathrm{~Hz}), 21.3 .{ }^{31} \mathrm{P} \mathrm{NMR}\left(\mathrm{CDCl}_{3}, 121 \mathrm{MHz}\right) \delta 48.6$. HRMS-ESI calcd for $\left[\mathrm{M}-\mathrm{BAr}_{\mathrm{F}}\right]^{+}:$859.3366, found: 859.3373. $[\alpha]_{\mathrm{D}}^{25}=-5.00\left(c=0.1\right.$ in $\left.\mathrm{CHCl}_{3}\right)$.

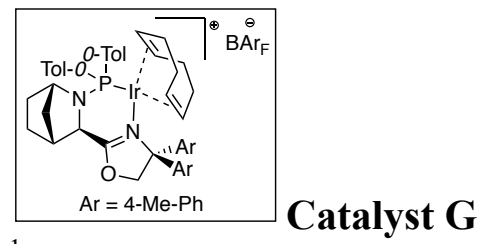

${ }^{1} \mathrm{H} \mathrm{NMR}\left(\mathrm{CDCl}_{3}, 400 \mathrm{MHz}\right) \delta 7.75-7.67(\mathrm{~m}, 8 \mathrm{H}), 7.55-7.49(\mathrm{~m}, 4 \mathrm{H}), 7.43(\mathrm{dt}, J=6.5$, $2.2 \mathrm{~Hz}, 2 \mathrm{H}), 7.37(\mathrm{~d}, J=8.1 \mathrm{~Hz}, 2 \mathrm{H}), 7.31-7.24(\mathrm{~m}, 2 \mathrm{H}), 7.25-7.15(\mathrm{~m}, 3 \mathrm{H}), 7.08(\mathrm{~d}, J$ $=8.0 \mathrm{~Hz}, 2 \mathrm{H}), 6.90(\mathrm{dt}, J=16.8,9.0 \mathrm{~Hz}, 2 \mathrm{H}), 6.80(\mathrm{dd}, J=15.1,8.1 \mathrm{~Hz}, 3 \mathrm{H}), 5.12(\mathrm{~s}$, $1 \mathrm{H}), 4.94$ (q, $J=9.0 \mathrm{~Hz}, 2 \mathrm{H}), 4.62(\mathrm{~s}, 1 \mathrm{H}), 4.37$ (d, $J=2.5 \mathrm{~Hz}, 1 \mathrm{H}), 3.40(\mathrm{~s}, 1 \mathrm{H}), 3.33$ (dd, $J=14.5,7.2 \mathrm{~Hz}, 2 \mathrm{H}), 3.27(\mathrm{~s}, 2 \mathrm{H}), 3.05(\mathrm{~s}, 1 \mathrm{H}), 2.30(\mathrm{~s}, 3 \mathrm{H}), 2.26(\mathrm{~s}, 3 \mathrm{H}), 2.16$ (s, $3 \mathrm{H}), 2.13-1.94(\mathrm{~m}, 2 \mathrm{H}), 1.83-1.66(\mathrm{~m}, 4 \mathrm{H}), 1.58(\mathrm{~s}, 2 \mathrm{H}), 1.50-1.23(\mathrm{~m}, 4 \mathrm{H}), 1.17-$ $1.01(\mathrm{~m}, 2 \mathrm{H}), 0.75-0.64(\mathrm{~m}, 1 \mathrm{H}) .{ }^{13} \mathrm{C} \mathrm{NMR}\left(\mathrm{CDCl}_{3}, 100 \mathrm{MHz}\right) \delta 172.7,161.7(\mathrm{dd}, J=$ 99.7, 49.8 Hz), 141.5 (d, $J=14.1 \mathrm{~Hz}), 139.9,139.4$ (d, $J=14.1 \mathrm{~Hz}), 138.4,134.8,132.6$, $132.3(\mathrm{~d}, J=8.8 \mathrm{~Hz}), 132.1,131.5(\mathrm{~d}, J=47.0 \mathrm{~Hz}), 130.7,129.7,129.1-128.9(\mathrm{~m})$, $128.8-128.6(\mathrm{~m}), 128.4,127.9,126.7$ (d, $J=7.7 \mathrm{~Hz}), 126.3$ (d, $J=9.1 \mathrm{~Hz}), 126.1$, $125.9,125.3(\mathrm{~d}, J=59.9 \mathrm{~Hz}), 123.2,120.5,117.8-117.1(\mathrm{~m}), 90.0(\mathrm{~d}, J=8.5 \mathrm{~Hz}), 86.6$, 81.7, $78.1(\mathrm{~d}, J=16.8 \mathrm{~Hz}), 69.1,63.7(\mathrm{~d}, J=10.8 \mathrm{~Hz}), 62.0,58.1,40.4,39.5,36.1,34.1$, 29.7, 29.3, 28.9, 27.1, 25.1 (d, $J=52.5 \mathrm{~Hz}), 22.3,20.9$ (d, $J=7.5 \mathrm{~Hz}), 14.1 .{ }^{31} \mathrm{P} \mathrm{NMR}$ $\left(\mathrm{CDCl}_{3}, 162 \mathrm{MHz}\right) \delta$ 48.4. HRMS-ESI; $\mathrm{m} / z$ [M-BAr $]^{+}$Calcd. for $\mathrm{C}_{45} \mathrm{H}_{51} \mathrm{IrN}_{2} \mathrm{OP}=$ 859.3366. Found: 859.3369. $[\alpha]^{25}=-13.00\left(c=0.1\right.$ in $\left.\mathrm{CHCl}_{3}\right)$.

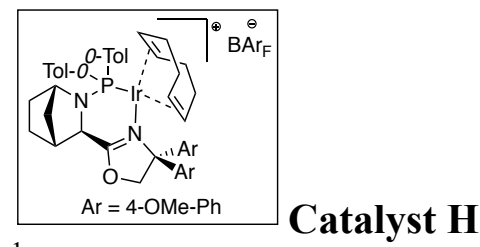

${ }^{1} \mathrm{H}$ NMR $\left(\mathrm{CDCl}_{3}, 400 \mathrm{MHz}\right) \delta 7.75(\mathrm{dd}, J=5.3,2.7 \mathrm{~Hz}, 8 \mathrm{H}), 7.56(\mathrm{~s}, 4 \mathrm{H}), 7.46(\mathrm{dd}, J=$ 8.8, $2.9 \mathrm{~Hz}, 4 \mathrm{H}), 7.36-7.17(\mathrm{~m}, 5 \mathrm{H}), 6.98(\mathrm{~d}, J=7.4 \mathrm{~Hz}, 1 \mathrm{H}), 6.95-6.86(\mathrm{~m}, 2 \mathrm{H}), 6.86$ 
- $6.79(\mathrm{~m}, 2 \mathrm{H}), 6.53(\mathrm{dd}, J=8.8,2.6 \mathrm{~Hz}, 2 \mathrm{H}), 5.15(\mathrm{~s}, 1 \mathrm{H}), 5.02-4.90(\mathrm{~m}, 2 \mathrm{H}), 4.67$ (s, $1 \mathrm{H}), 4.40(\mathrm{~d}, J=2.6 \mathrm{~Hz}, 1 \mathrm{H}), 3.78(\mathrm{~s}, 6 \mathrm{H}), 3.44(\mathrm{~s}, 3 \mathrm{H}), 3.30(\mathrm{~s}, 3 \mathrm{H}), 3.08(\mathrm{~s}, 1 \mathrm{H}), 2.43-$ $2.27(\mathrm{~m}, 1 \mathrm{H}), 2.20(\mathrm{~d}, J=2.3 \mathrm{~Hz}, 3 \mathrm{H}), 2.07(\mathrm{dd}, J=41.4,11.1 \mathrm{~Hz}, 2 \mathrm{H}), 1.93-1.69(\mathrm{~m}$, 4H), $1.60(\mathrm{~d}, J=10.4 \mathrm{~Hz}, 1 \mathrm{H}), 1.53-1.39(\mathrm{~m}, 2 \mathrm{H}), 1.22-1.04(\mathrm{~m}, 3 \mathrm{H}), 0.93-0.83(\mathrm{~m}$, $1 \mathrm{H}), 0.74(\mathrm{t}, J=10.9 \mathrm{~Hz}, 1 \mathrm{H}) .{ }^{13} \mathrm{C} \mathrm{NMR}\left(\mathrm{CDCl}_{3}, 100 \mathrm{MHz}\right) \delta 172.7,161.9(\mathrm{dd}, J=99.6$, $49.8 \mathrm{~Hz}$ ), 160.4, 159.4, 141.7 (d, $J=13.6 \mathrm{~Hz}), 139.6$ (d, $J=14.0 \mathrm{~Hz}), 135.0,133.1-$ $132.4(\mathrm{~m}), 132.3,131.8,131.3,131.0,129.8,129.5,129.3,129.3-129.1(\mathrm{~m}), 128.9$ (dd, $J=5.8,2.8 \mathrm{~Hz}), 128.8,128.6(\mathrm{dd}, J=5.8,2.8 \mathrm{~Hz}), 127.7,126.9(\mathrm{~d}, J=7.7 \mathrm{~Hz}), 126.5(\mathrm{~d}$, $J=8.9 \mathrm{~Hz}), 126.1,125.8,125.2,123.4,120.7,117.6$ (p, $J=4.2 \mathrm{~Hz}), 114.4,113.9,69.2$, $63.9(\mathrm{~d}, J=11.0 \mathrm{~Hz}), 62.1,58.2(\mathrm{~d}, J=5.1 \mathrm{~Hz}), 55.4(\mathrm{~d}, J=5.7 \mathrm{~Hz}), 40.6(\mathrm{~d}, J=5.6 \mathrm{~Hz})$, 39.6,36.3, 34.2, 33.0 (d, $J=191.2 \mathrm{~Hz}), 30.2-28.3(\mathrm{~m}), 27.2,25.3(\mathrm{~d}, J=45.4 \mathrm{~Hz}), 22.5$ $(\mathrm{d}, J=8.8 \mathrm{~Hz}), 14.3 .{ }^{31} \mathrm{P}$ NMR $\left(\mathrm{CDCl}_{3}, 162 \mathrm{MHz}\right) \delta 48.6$. HRMS-ESI; $\left.m / z . \mathrm{M}-\mathrm{BAr}_{\mathrm{F}}\right]^{+}$ Calcd. for $\mathrm{C}_{45} \mathrm{H}_{51} \mathrm{IrN}_{2} \mathrm{OP}=891.3264$. Found: 891.3261. $[\alpha]_{\mathrm{D}}^{25}=-19.00(c=0.1$ in $\mathrm{CHCl}_{3}$ ).

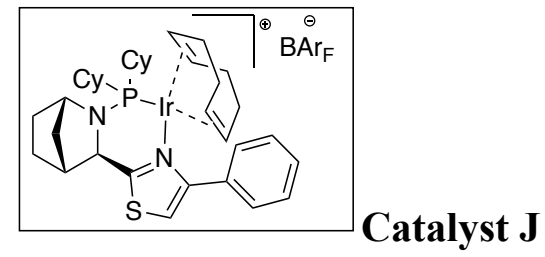

Orange colored crystalline solid. ${ }^{1} \mathrm{H}$ NMR $\left(\mathrm{CDCl}_{3}, 400 \mathrm{MHz}\right): \delta 8.20(\mathrm{dd}, J=7.3,2.2$ $\mathrm{Hz}, 2 \mathrm{H}), 7.71(\mathrm{p}, J=2.0 \mathrm{~Hz}, 8 \mathrm{H}), 7.57(\mathrm{dd}, J=5.9,1.5 \mathrm{~Hz}, 3 \mathrm{H}), 7.52(\mathrm{~s}, 4 \mathrm{H}), 7.49$ (s, $1 \mathrm{H}), 4.85(\mathrm{~s}, 1 \mathrm{H}), 4.62(\mathrm{~s}, 1 \mathrm{H}), 4.44(\mathrm{~d}, J=2.3 \mathrm{~Hz}, 1 \mathrm{H}), 3.65(\mathrm{~s}, 1 \mathrm{H}), 3.21(\mathrm{~s}, 1 \mathrm{H}), 2.99$ $(\mathrm{s}, 1 \mathrm{H}), 2.89$ (p, $J=7.5 \mathrm{~Hz}, 1 \mathrm{H}), 2.53(\mathrm{q}, J=13.2 \mathrm{~Hz}, 1 \mathrm{H}), 2.39-2.30(\mathrm{~m}, 1 \mathrm{H}), 2.22-$ $2.13(\mathrm{~m}, 2 \mathrm{H}), 2.07(\mathrm{dd}, J=19.3,8.6 \mathrm{~Hz}, 2 \mathrm{H}), 2.01-1.77(\mathrm{~m}, 9 \mathrm{H}), 1.77-1.57(\mathrm{~m}, 7 \mathrm{H})$, $1.40-1.21(\mathrm{~m}, 8 \mathrm{H}), 1.15-1.05(\mathrm{~m}, 3 \mathrm{H}), 0.97-0.86(\mathrm{~m}, 2 \mathrm{H}), 0.75-0.65(\mathrm{~m}, 1 \mathrm{H}) .{ }^{13} \mathrm{C}$ NMR $\left(\mathrm{CDCl}_{3}, 100 \mathrm{MHz}\right) \delta 173.7,161.8(\mathrm{dd}, J=99.9,49.7 \mathrm{~Hz}), 157.2,134.9,132.4$, $131.0,129.5,129.3-129.1(\mathrm{~m}), 129.0$ - $128.8(\mathrm{~m}), 127.4,126.0,123.3,120.6,117.8-$ $117.4(\mathrm{~m}), 117.3,93.6(\mathrm{~d}, J=8.1 \mathrm{~Hz}), 85.6(\mathrm{~d}, J=15.2 \mathrm{~Hz}), 71.1,68.3(\mathrm{~d}, J=7.7 \mathrm{~Hz})$, 63.8, $59.8(\mathrm{~d}, J=5.3 \mathrm{~Hz}), 42.7(\mathrm{~d}, J=6.1 \mathrm{~Hz}), 38.7-38.4(\mathrm{~m}), 37.8(\mathrm{~d}, J=3.7 \mathrm{~Hz}), 35.4$, 34.9, 33.3, 29.4 (d, $J=9.3 \mathrm{~Hz}), 28.0(\mathrm{~d}, J=4.3 \mathrm{~Hz}), 27.3(\mathrm{~d}, J=9.2 \mathrm{~Hz}), 26.6,26.5$, 26.4, $26.4-26.3(\mathrm{~m}), 26.2-26.2(\mathrm{~m}), 26.1,24.2(\mathrm{~d}, J=2.5 \mathrm{~Hz}) \cdot{ }^{31} \mathrm{P}$ NMR $\left(\mathrm{CDCl}_{3}, 121\right.$ $\mathrm{MHz}) \delta$ 60.3. HRMS-ESI calcd for [M-BArF] $]^{+}$753.2978, found: $735.2984 .[\alpha]^{25}=$ $+88.00\left(c=0.1\right.$ in $\left.\mathrm{CHCl}_{3}\right)$.

Some compounds reported in this manuscript were synthesized following procedures reported in literature. The spectroscopic data is in agreement with literature.

\section{Literature references of known compounds}

\begin{tabular}{l}
\hline $\mathrm{MeO}{ }_{2} \mathrm{C}$ \\
$\begin{array}{l}\text { Synthesis of Substituted Succinic Diesters. Adv. Synth. Catal.2015, 357 (1), } \\
\text { 177-184. }\end{array}$
\end{tabular}




\begin{tabular}{|c|c|}
\hline & $\begin{array}{l}\text { Concellón, J. M.; Bardales, E. Synthesis of }(E)-\alpha, \beta \text {-Unsaturated Esters with } \\
\text { Total or High Diastereoselectivity from } \alpha, \beta \text {-Epoxyesters. Org. Lett. 2002, 4, } \\
\text { 189-191. }\end{array}$ \\
\hline & $\begin{array}{l}\text { Durkin, K.; Liotta, D.; Rancourt, J.; Lavallee, J. F.; Boisvert, L.; Guindon, Y. } \\
\text { Stereoselective hydrogen-transfer reactions involving acyclic radicals. A study } \\
\text { of radical conformations using semiempirical calculations. J. Am. Chem. Soc. } \\
\text { 1992, 114, 4912-4914. }\end{array}$ \\
\hline & $\begin{array}{l}\text { Tsuda, T.; Yoshida, T.; Saegusa, T. Palladium (0)-catalyzed coupling reaction } \\
\text { of lithium (.alpha.-carbalkoxyvinyl) cuprates with organic halides. J. Org. } \\
\text { Chem. 1988, 53, 607-610. }\end{array}$ \\
\hline & $\begin{array}{l}\text { Malmedy, F.; Wirth, T. Stereoselective Ketone Rearrangements with } \\
\text { Hypervalent Iodine Reagents. Chem. Eur. J. 2016, 22, 16072-16077. }\end{array}$ \\
\hline & $\begin{array}{l}\text { Wang, P.; Liu, C.-R.; Sun, X.-L.; Chen, S.-S.; Li, J.-F.; Xie, Z.; Tang, Y. A } \\
\text { newly-designed PE-supported arsine for efficient and practical catalytic Wittig } \\
\text { olefination. Chem.Commun. 2012, 48, 290-292. }\end{array}$ \\
\hline & $\begin{array}{l}\text { Stensaas, K. L.; McCarty, B. V.; Touchette, N. M.; Brock, J. B.A comparison } \\
\text { of hydrogen bonding solvent effects on the singlet oxygen reactions of allyl } \\
\text { and vinyl sulfides, sulfoxides, and sulfones. Tetrahedron, 2006, 62, 10683- } \\
10687 \text {. }\end{array}$ \\
\hline & $\begin{array}{l}\text { Heß, T.; Raabe, G.; Gais, H.-J. Lithium-Titanium Exchange of Tertiary } \alpha- \\
\text { Sulfonyl Carbanions: Synthesis, Structure, Dynamics and Reactivity of Bis (1- } \\
\text { sulfonylalkyl) titaniums. Eur.J. Org. Chem. 2014, 7134-7147. }\end{array}$ \\
\hline & $\begin{array}{l}\text { Zhang, Q.-Q.; Xie, J.-H.; Yang, X.-H.; Xie, J.-B.; Zhou, Q.-L. Iridium- } \\
\text { Catalyzed Asymmetric Hydrogenation of } \alpha \text {-Substituted } \alpha, \beta \text {-Unsaturated } \\
\text { Acyclic Ketones: Enantioselective Total Synthesis of (-)-Mesembrine. Org. } \\
\text { Lett. 2012, 14, 6158-6161. }\end{array}$ \\
\hline $\mathrm{Me}$ & $\begin{array}{l}\text { Oi, S.; Moro, M.; Ono, S.; Inoue, Y. Rhodium-catalyzed Conjugate Addition } \\
\text { of Arylstannanes to } \alpha, \beta \text {-Unsaturated Ketones and Esters. Chem. Lett. 1998, } 1 \text {, } \\
83-84 \text {. }\end{array}$ \\
\hline & $\begin{array}{l}\text { Lightburn, T. E.; Dombrowski, M. T.; Tan, K. L. Catalytic scaffolding ligands: } \\
\text { an efficient strategy for directing reactions. J. Am. Chem. Soc. 2008, 130, } \\
9210-9211 .\end{array}$ \\
\hline & $\begin{array}{l}\text { Ueno, Y.; Moriya, O.; Chino, K.; Watanabe, M.; Okawara, M. General } \\
\text { synthetic route to } \gamma \text {-butyrolactones via stereoselective radical cyclization by } \\
\text { organotin species. J. Chem. Soc. Perkin Trans. I. 1986, 1351-1356. }\end{array}$ \\
\hline
\end{tabular}

\section{Absolute configuration determination 7a. Di-ester containing compounds}



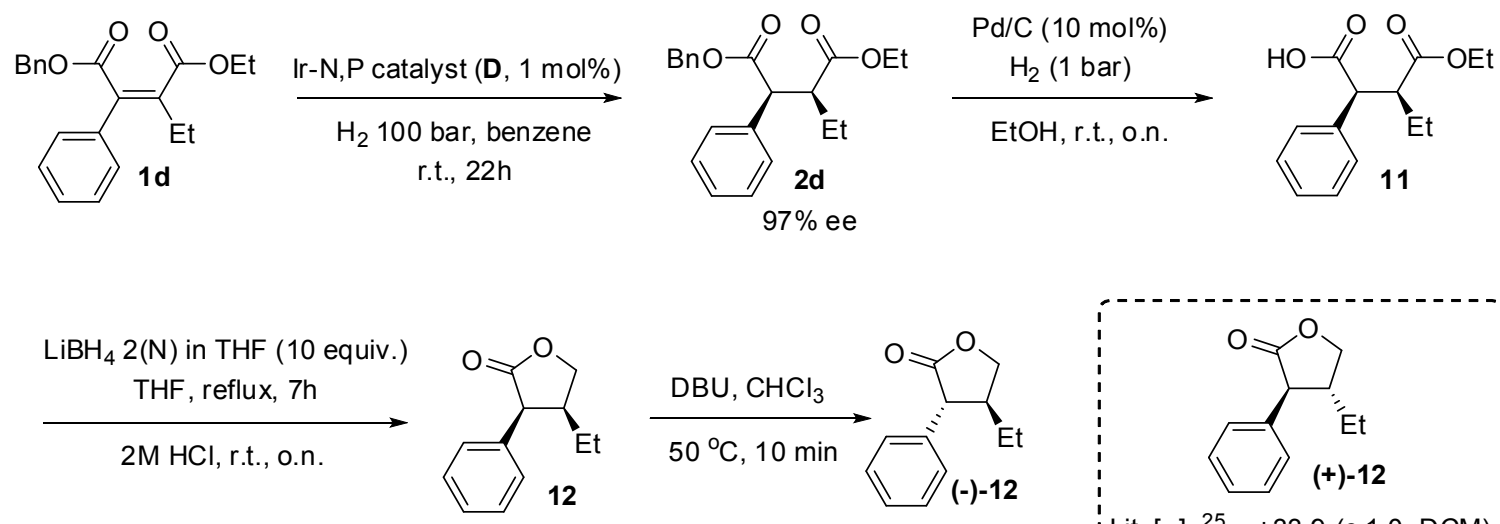

$95 \%$ Epimerization
$[\alpha]_{D}^{25}=-38.2($ c $0.12, D C M)$

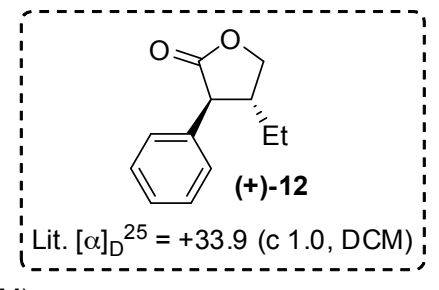

The absolute configuration of the hydrogenated product was determined by transformation of product 2d into known compound (-)-12. First, the benzylester in compound 2d was selectively cleaved off via hydrogenolysis to furnish compound $\mathbf{1 1}$. A chemoselective reduction of the ethyl ester using $\mathrm{LiBH}_{4}$ followed by cyclization under acidic conditions afforded cis-lactone 12. Finally, epimerization with DBU resulted in the trans-isomer of (-)-12. The absolute configuration of the other products have been tentatively assigned by comparison of elution order from HPLC, using a chiral stationary phase and the optical rotation of product $\mathbf{2 d}$.

7a. Trifluoromethyl containing compounds by single crystal X-ray diffraction Single crystal of $\mathbf{6 c}$ was grown, by dissolving in pentane and slow evaporation at room temperature. Detailed information for this crystal is available in CCDC 1907708.

\section{Chromatogram of the chiral compounds}

Compounds containing di-ester were converted to the di-alcholic compound by reducing the ester by DIBALH in THF at r.t. and separated by SFC (IA 5\% MeOH, Flow rate: 2 $\mathrm{mL} / \mathrm{min}$ ) as appeared below. 

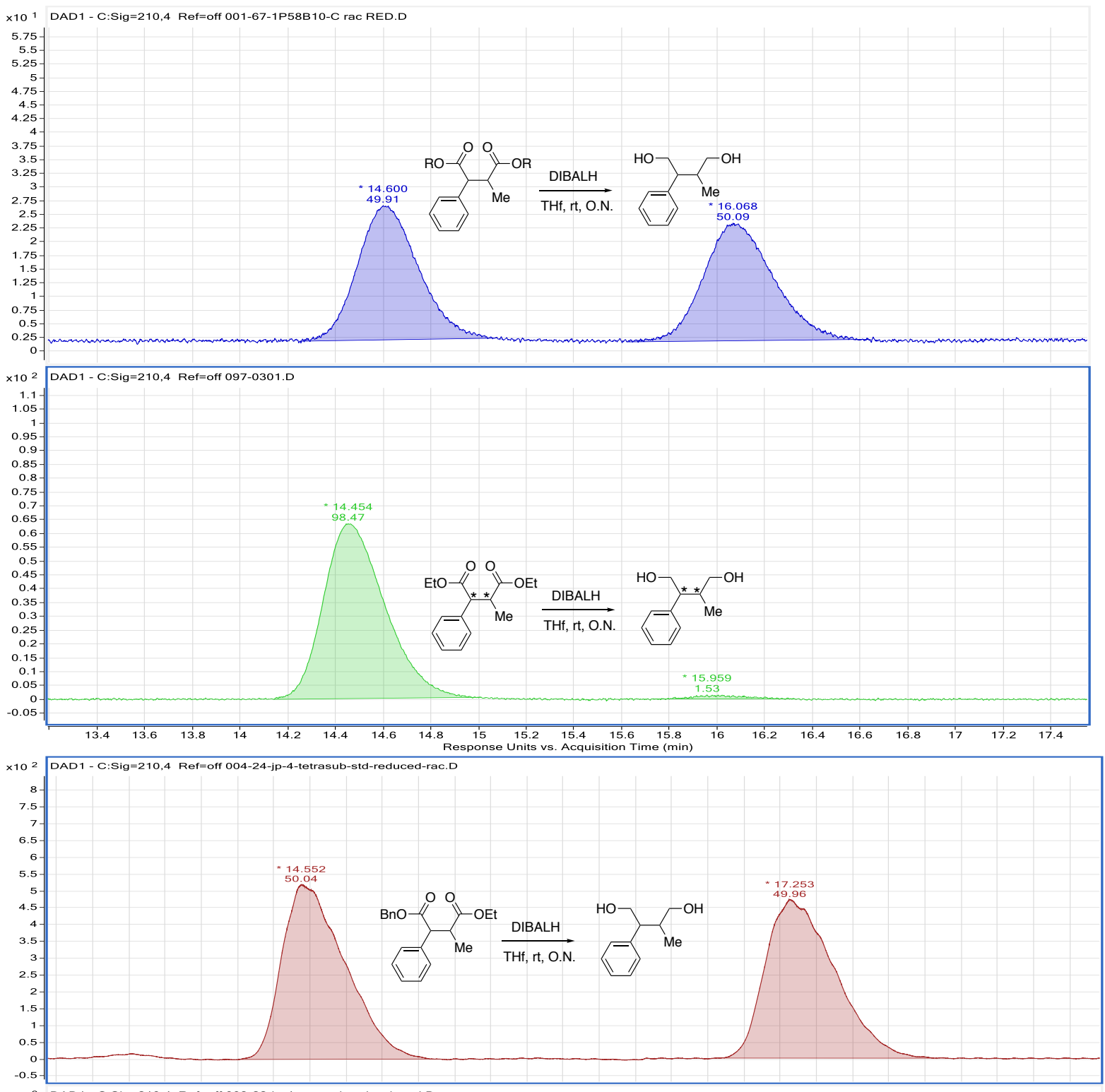

....

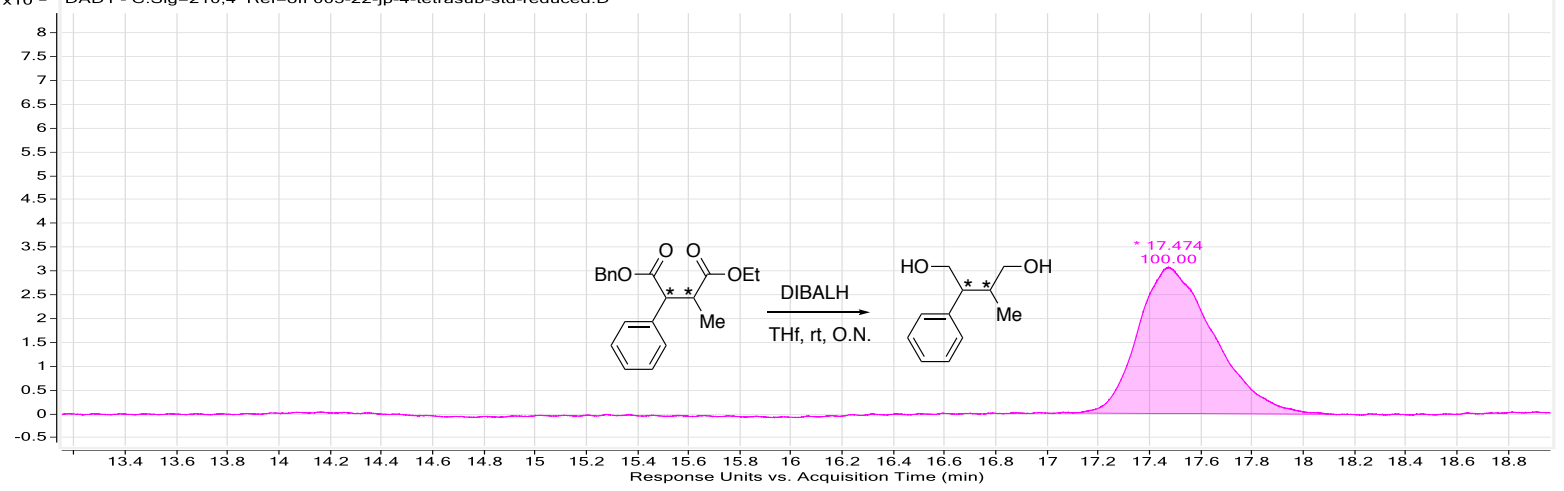



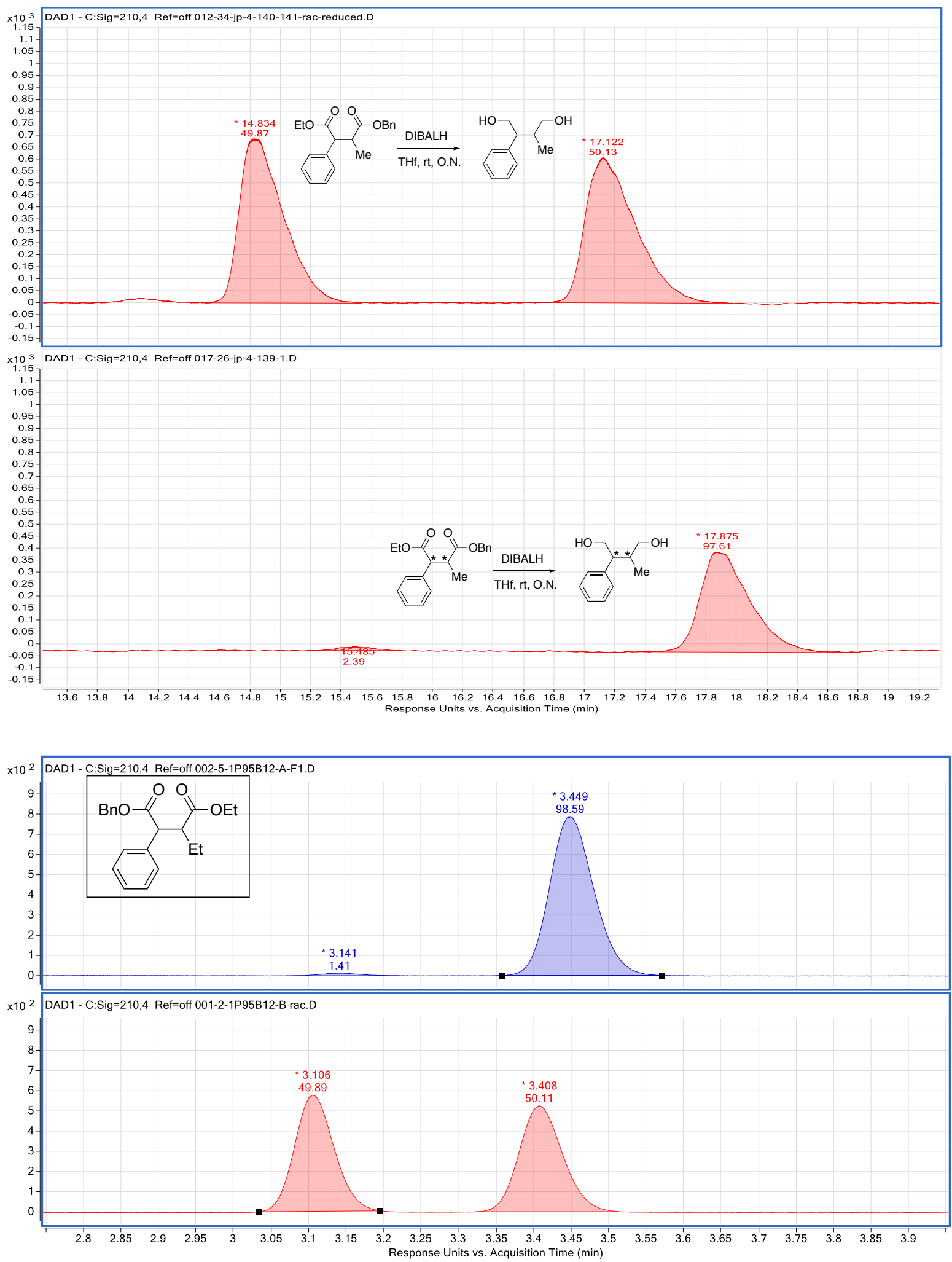

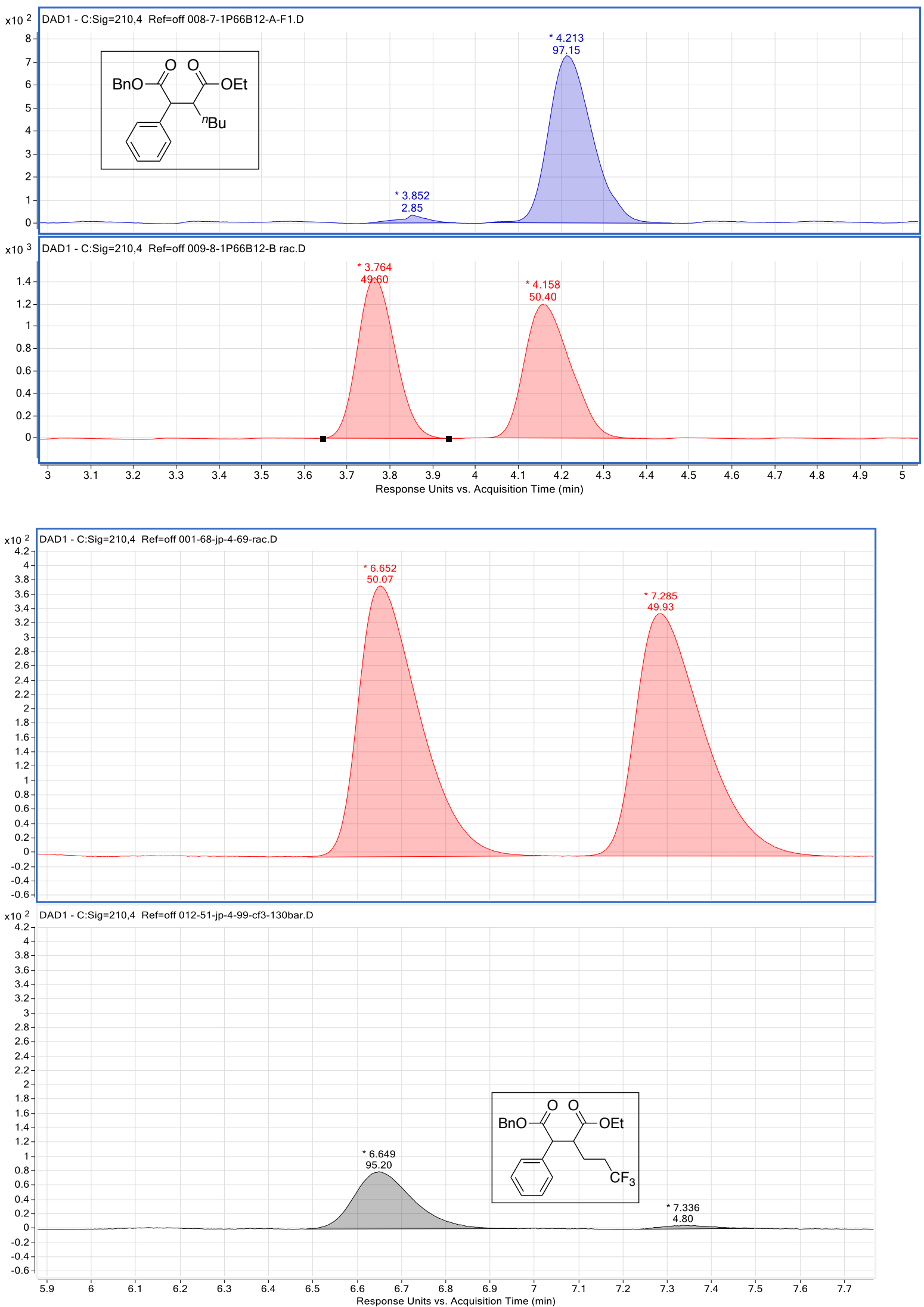

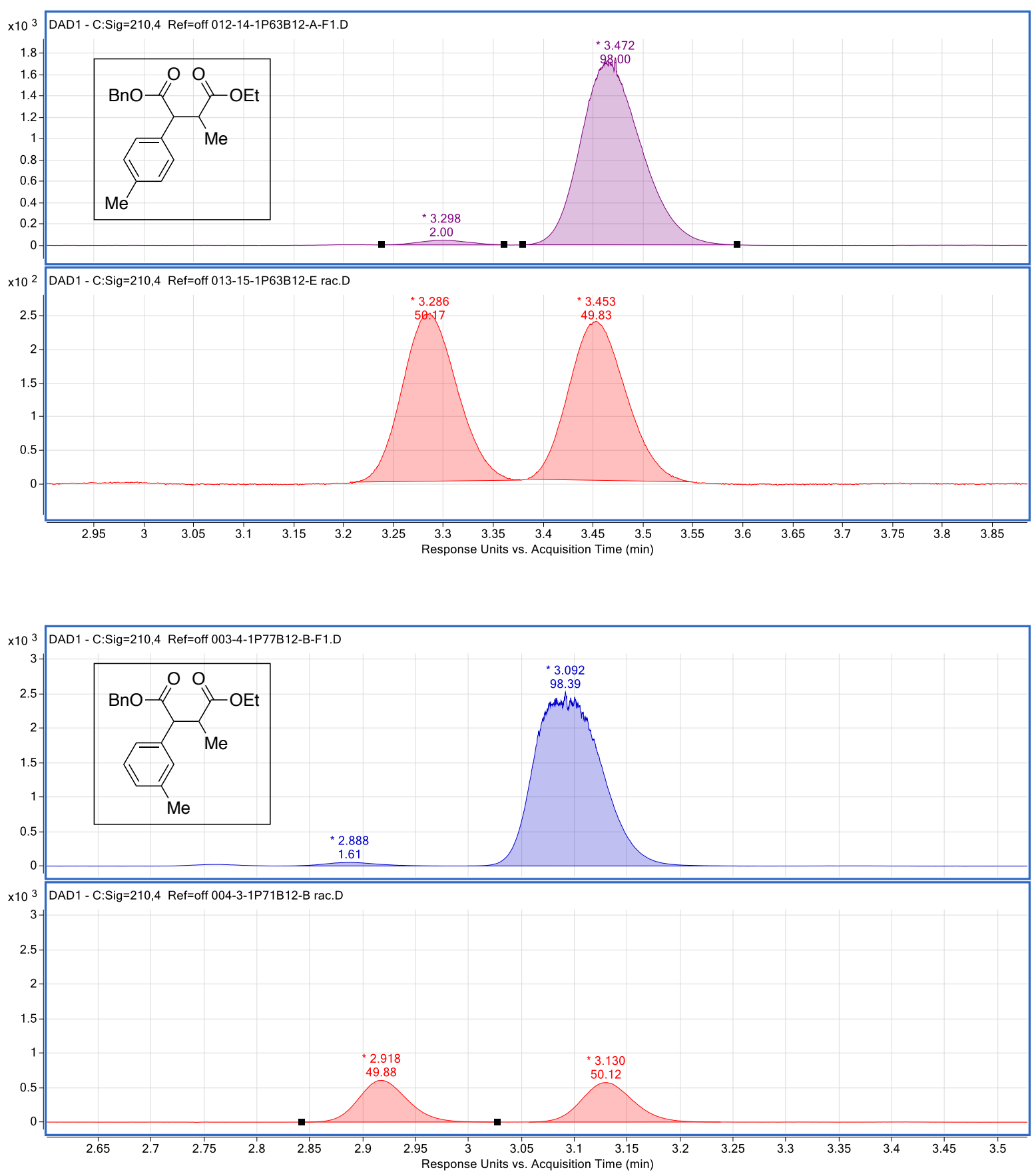

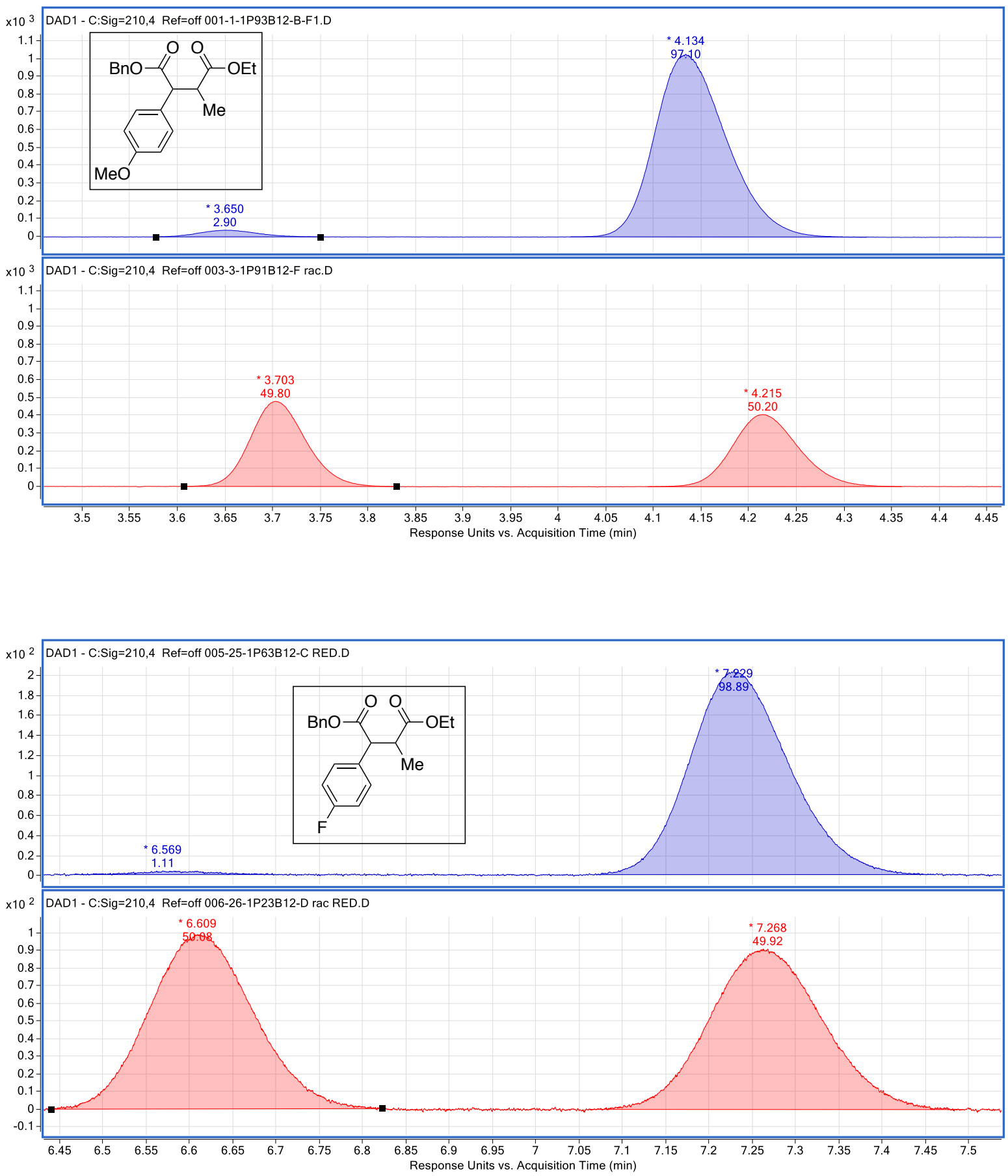

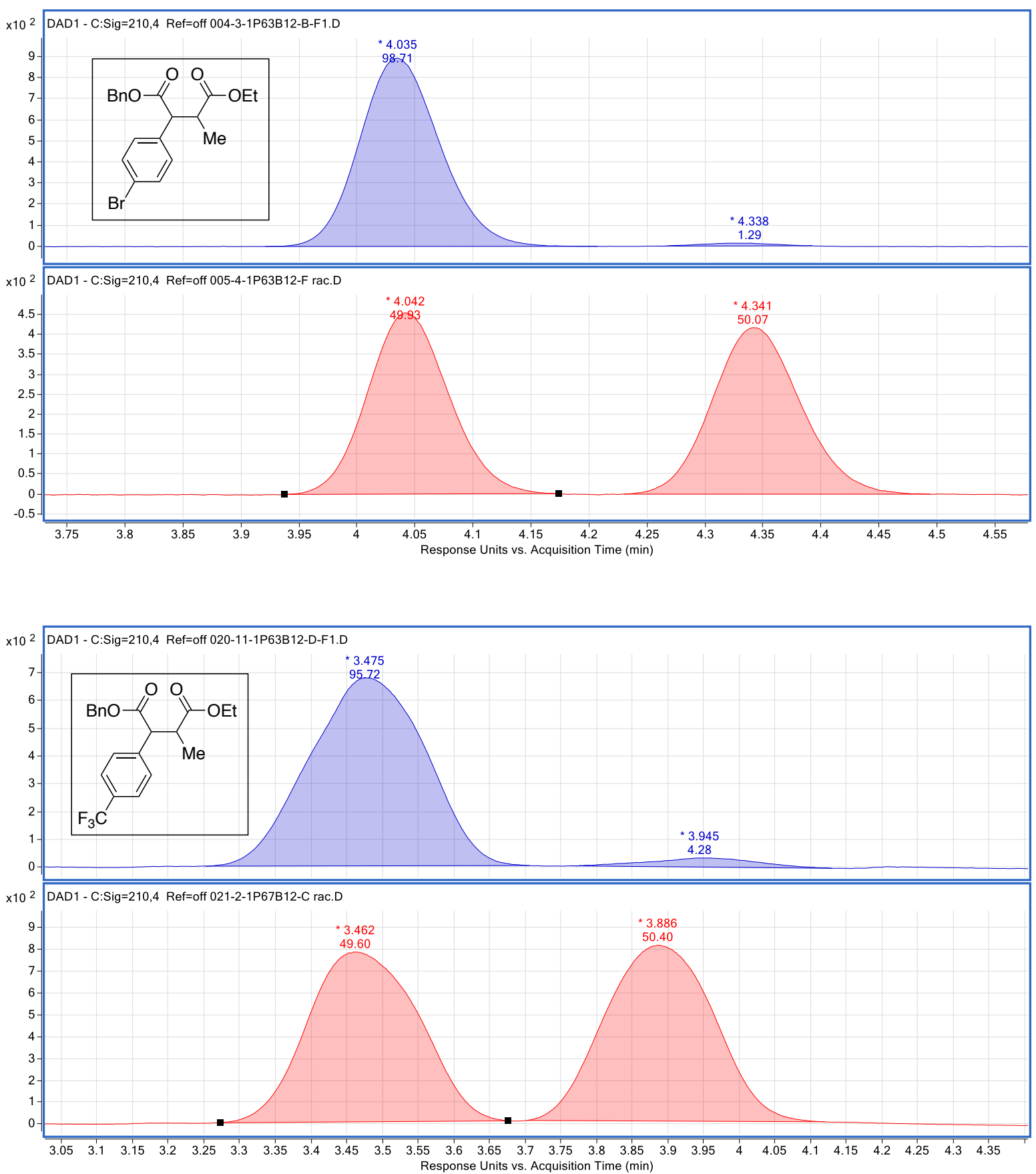


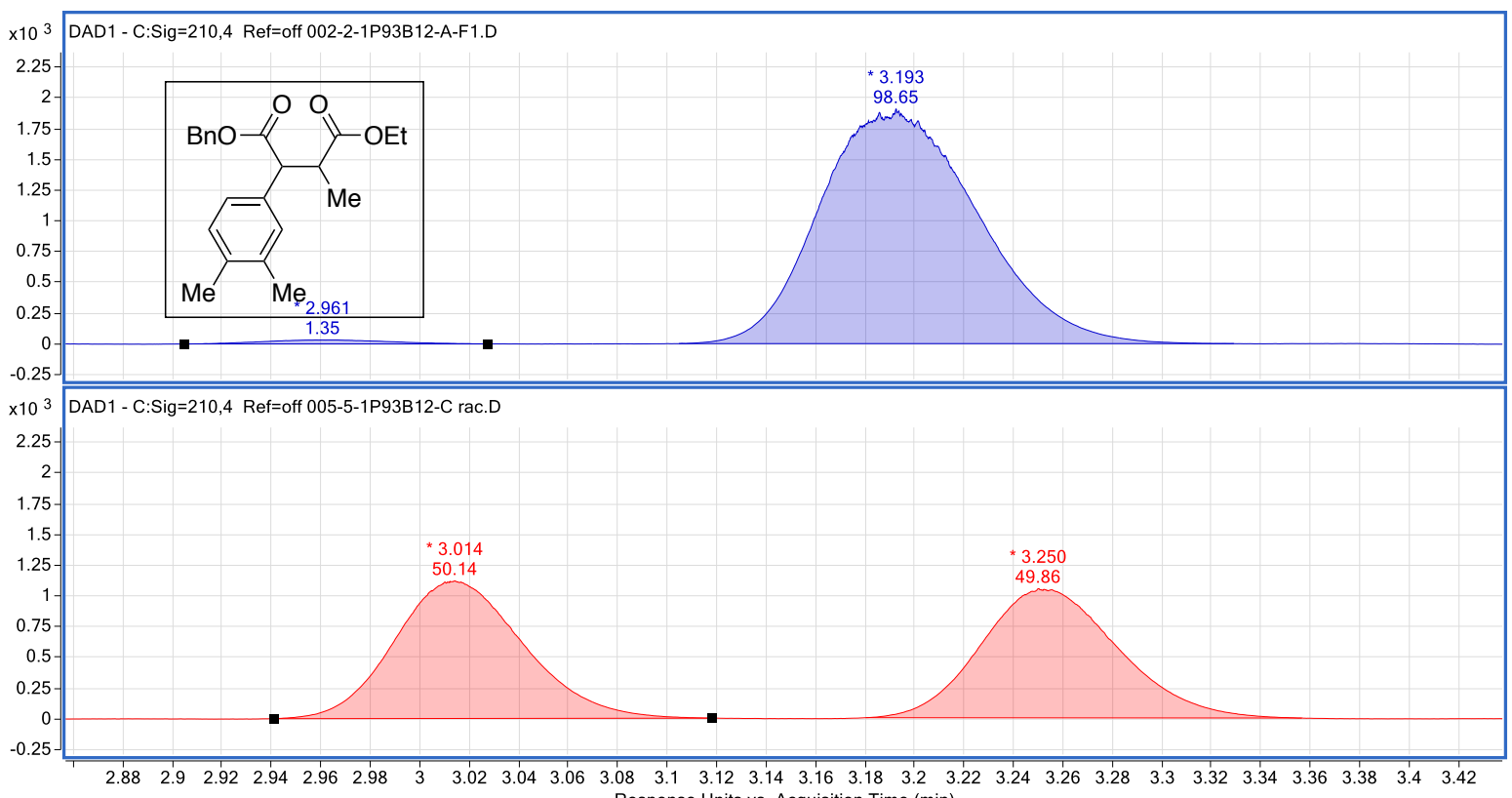

$\begin{array}{lllllllllllllllll}2.88 & 2.9 & 2.92 & 2.94 & 2.96 & 2.98 & 3 & 3.02 & 3.04 & 3.06 & \begin{array}{c}3.08 \\ \text { Response Units vs. Acquisition Time (min) }\end{array}\end{array}$
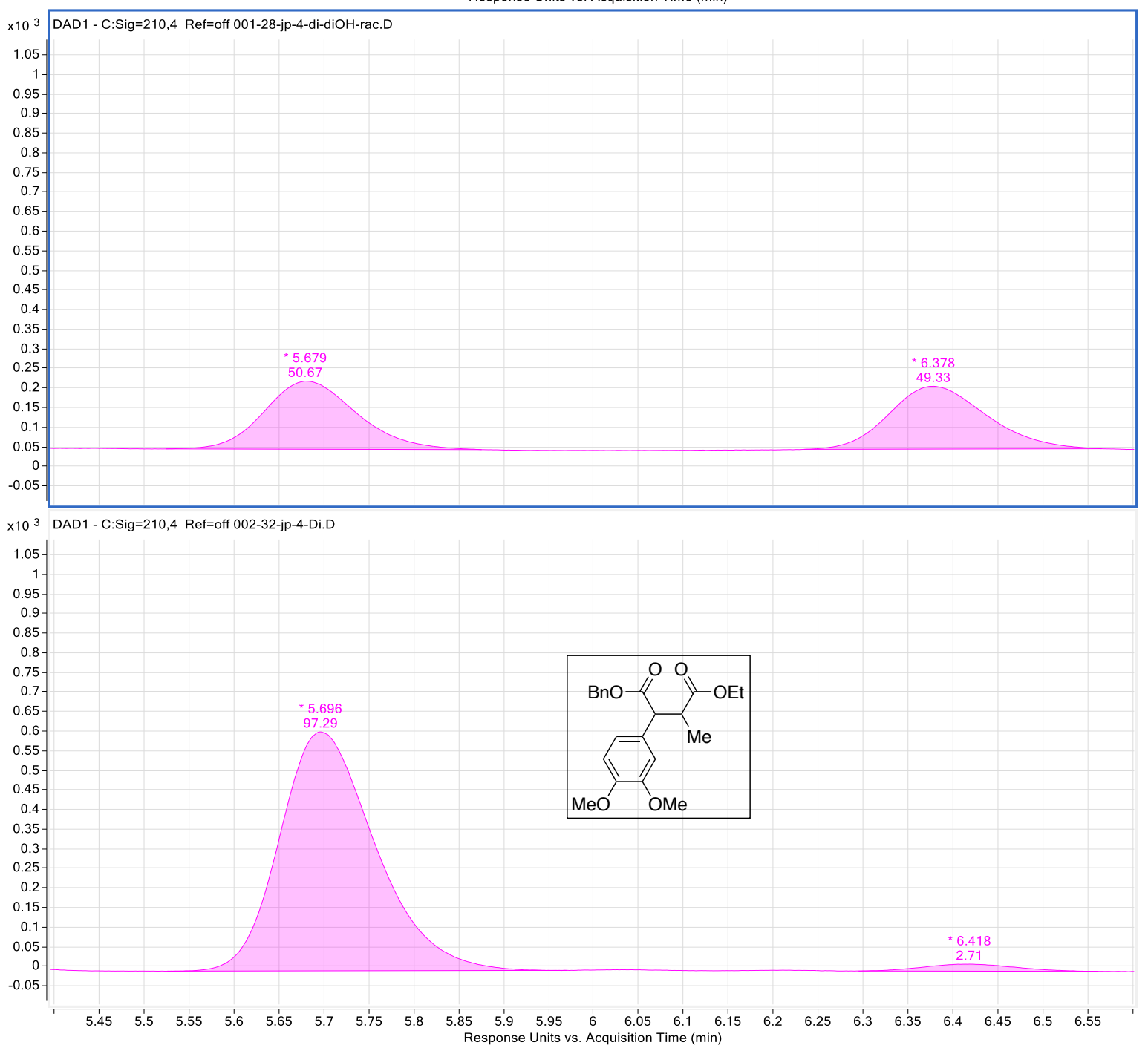

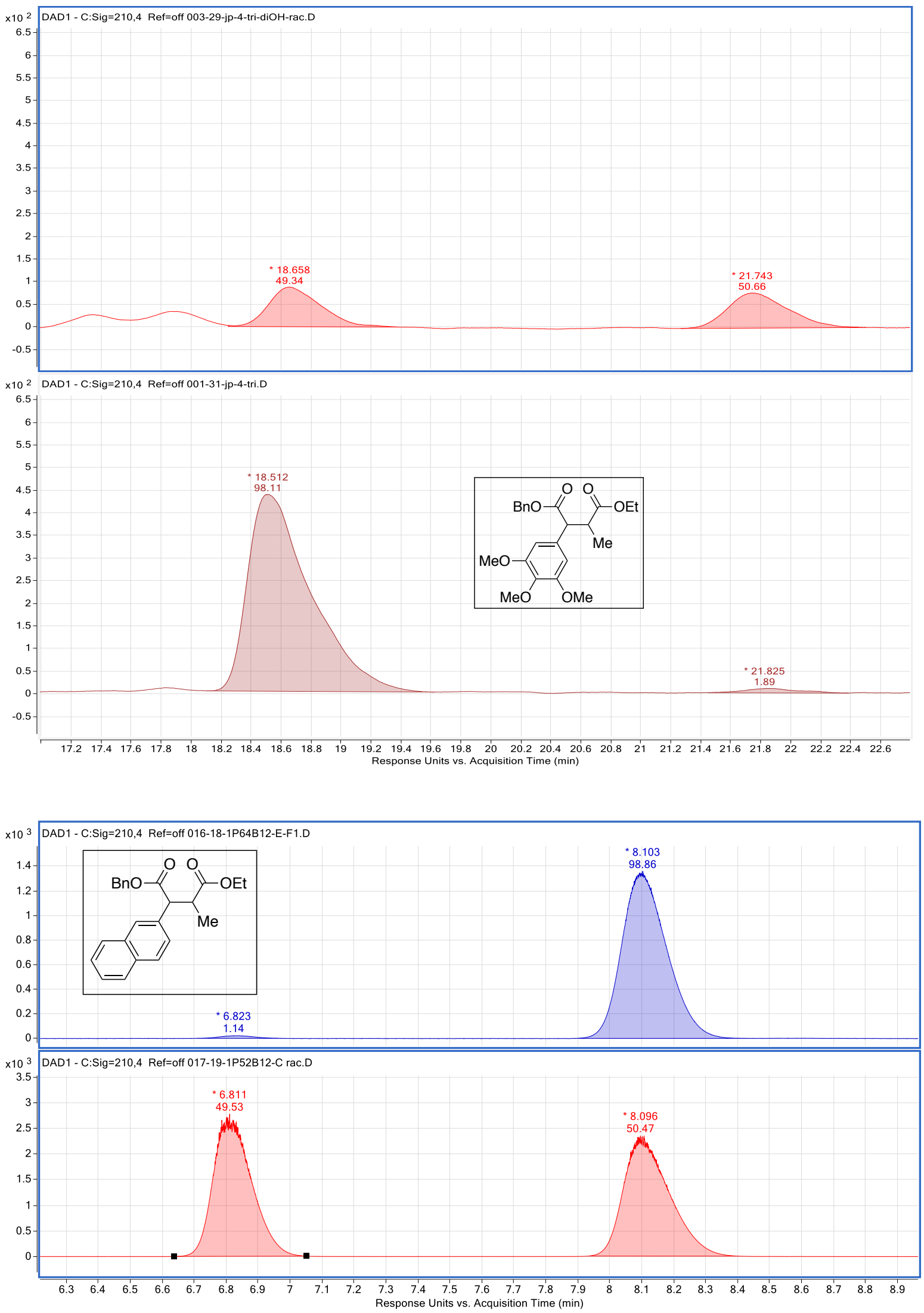

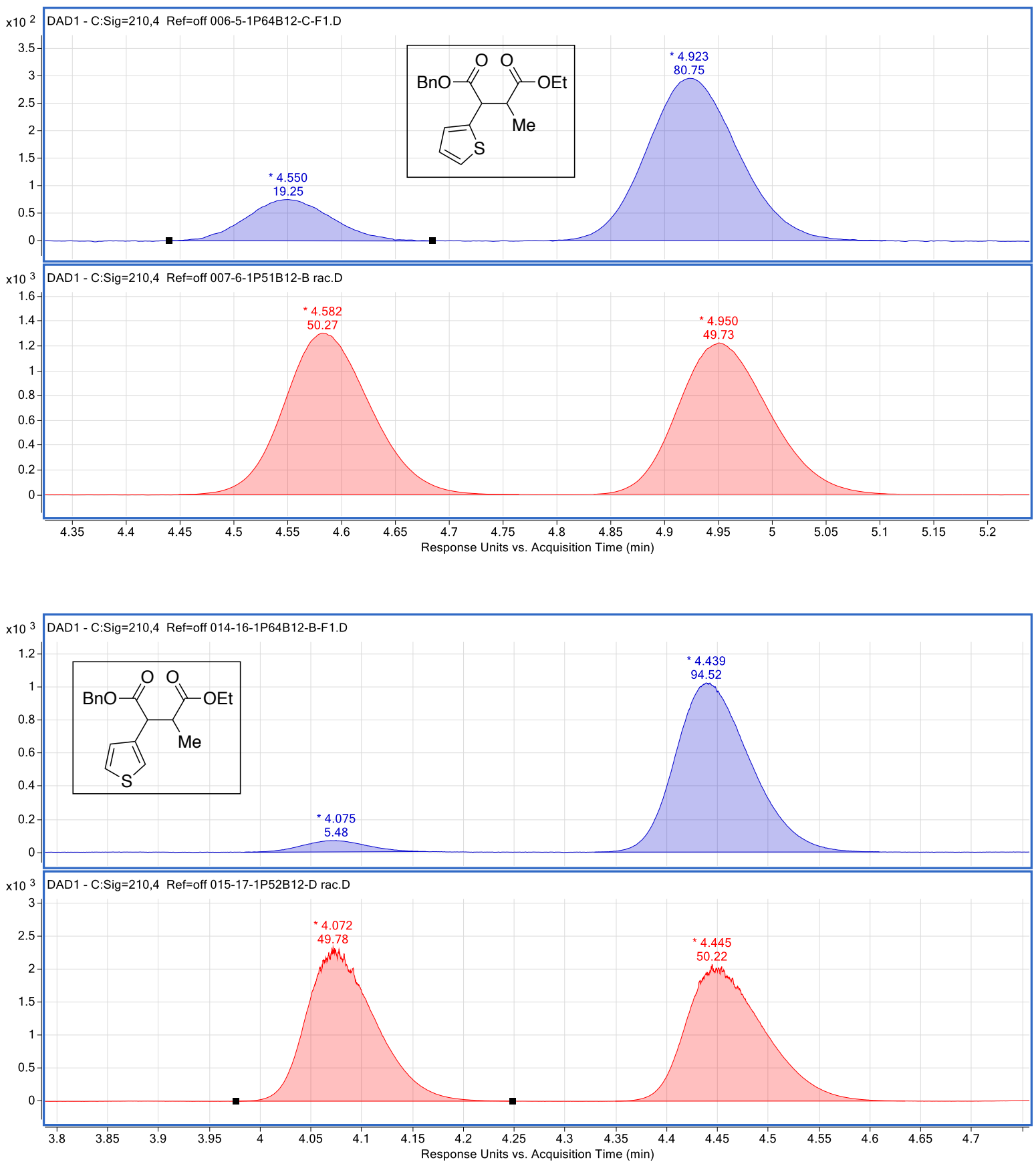

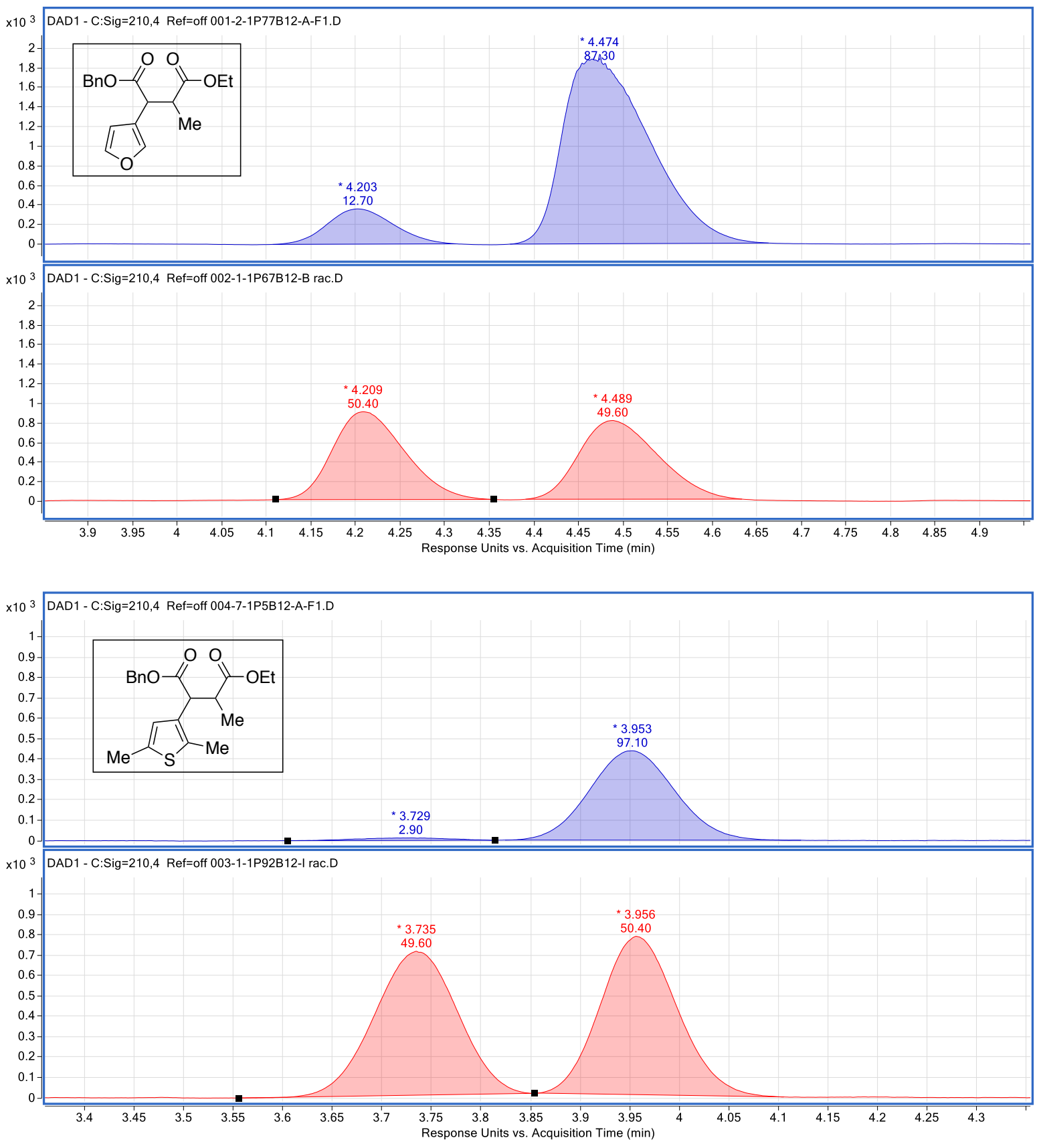

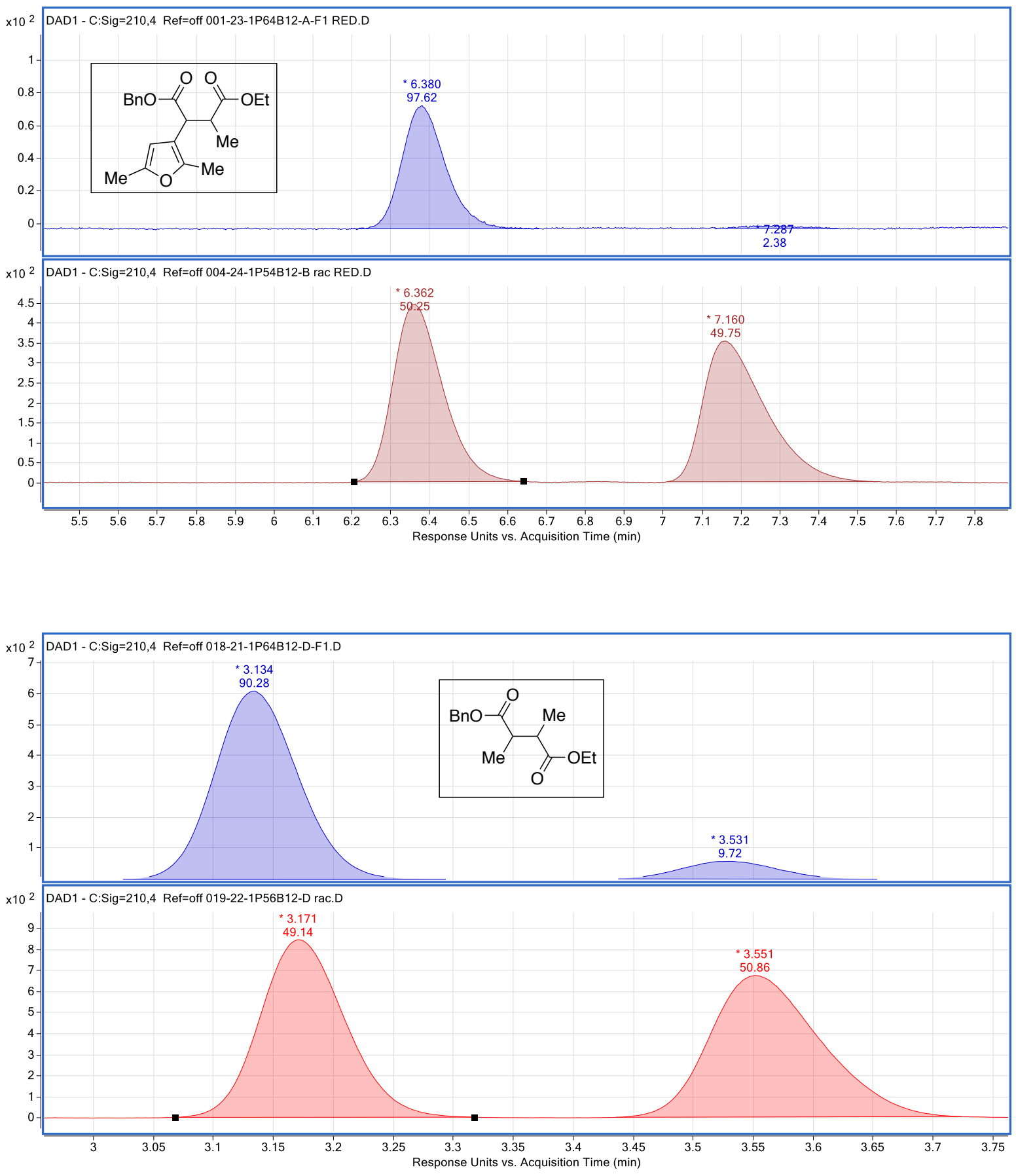
Chromatogram Plots
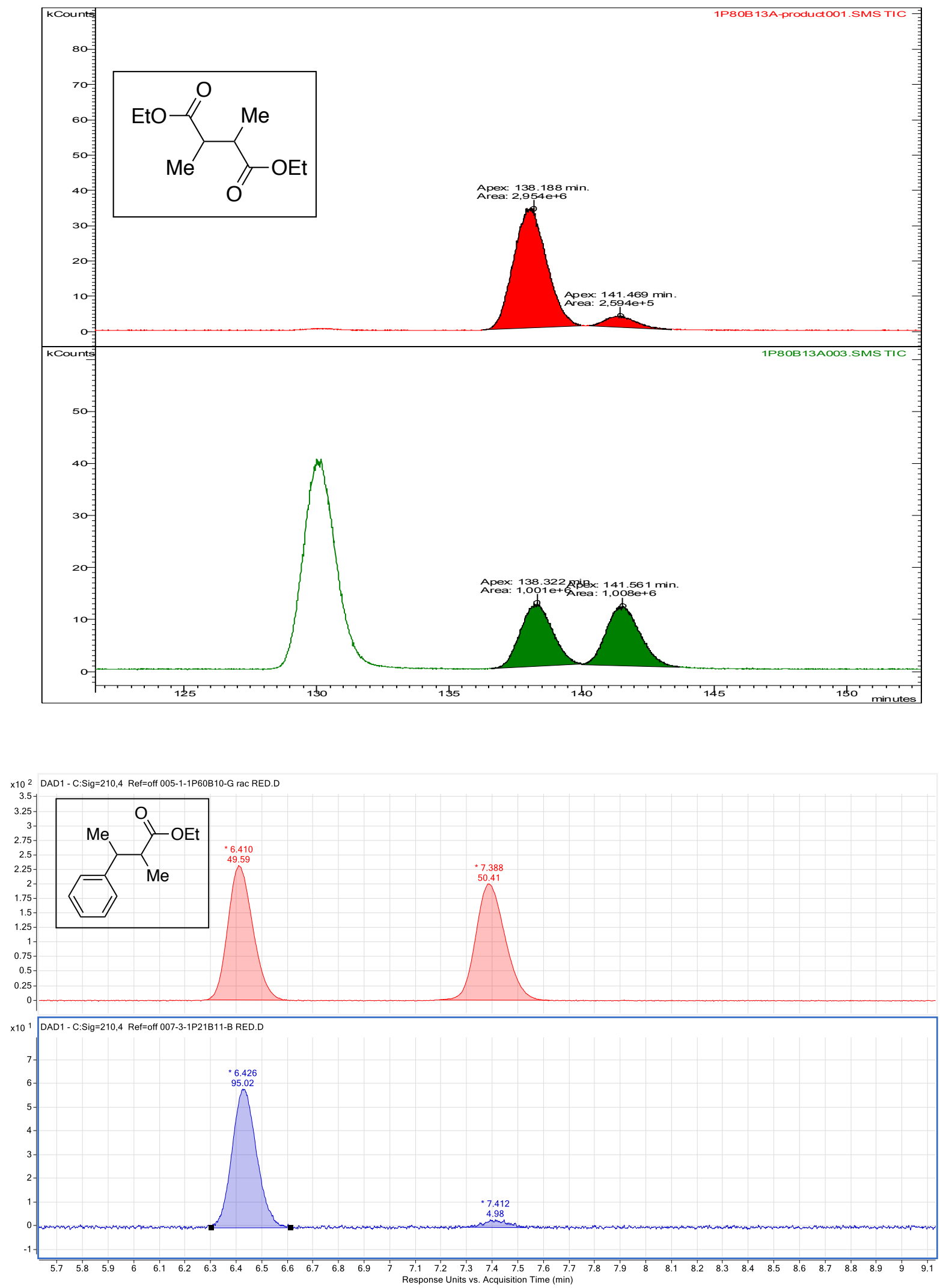

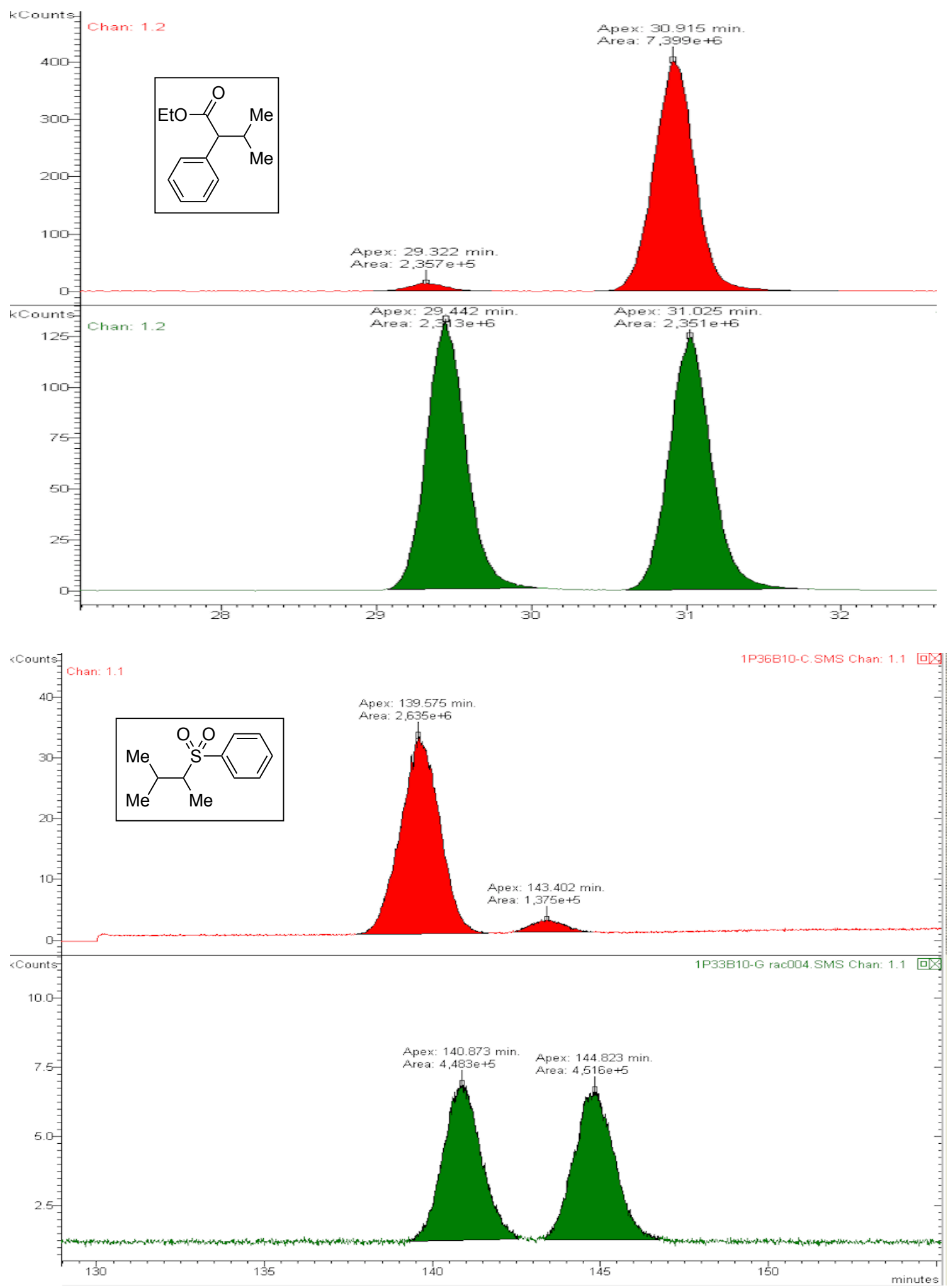

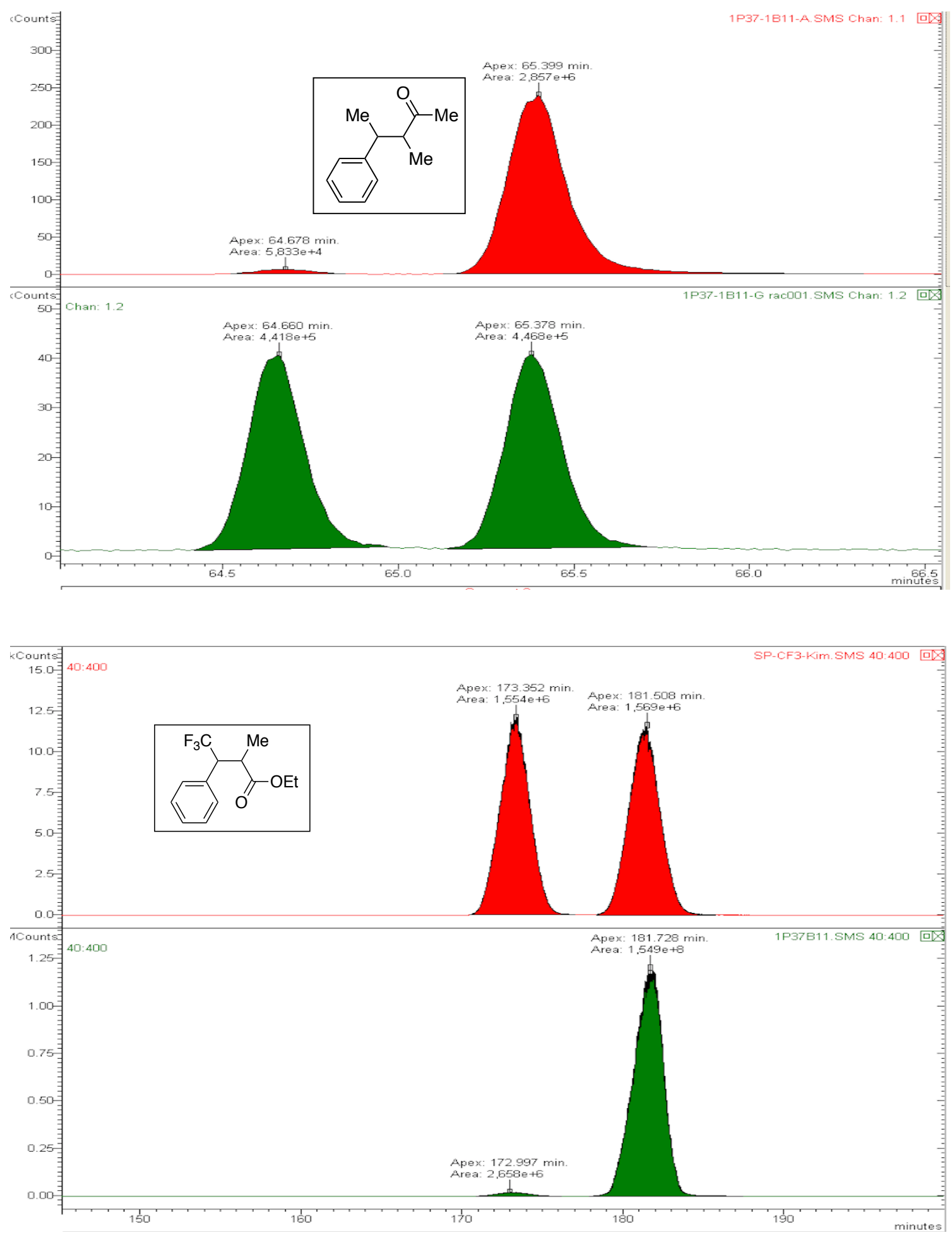

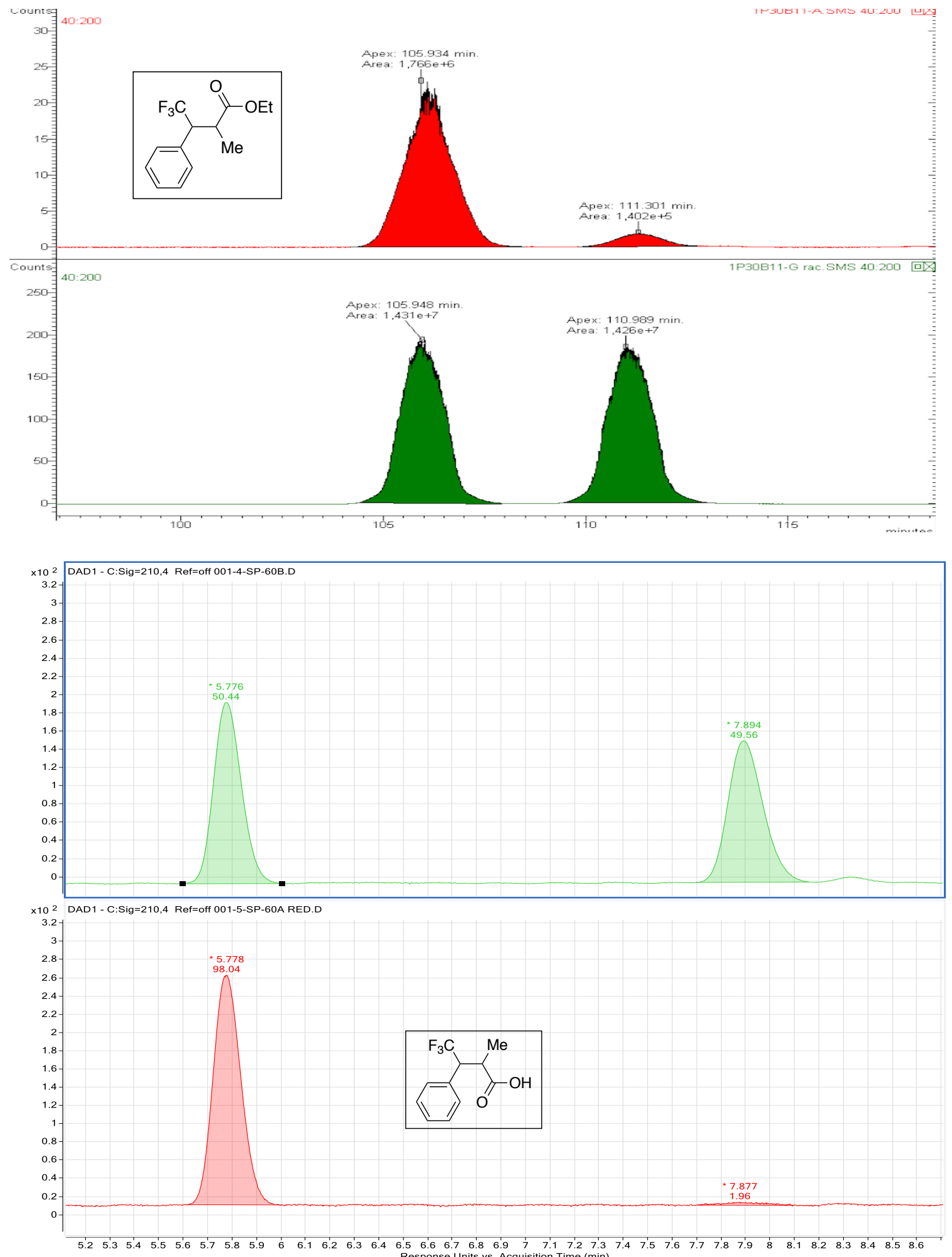
Print Date: 26 Mar 2019 09:44:12

\section{Chromatogram Plots}

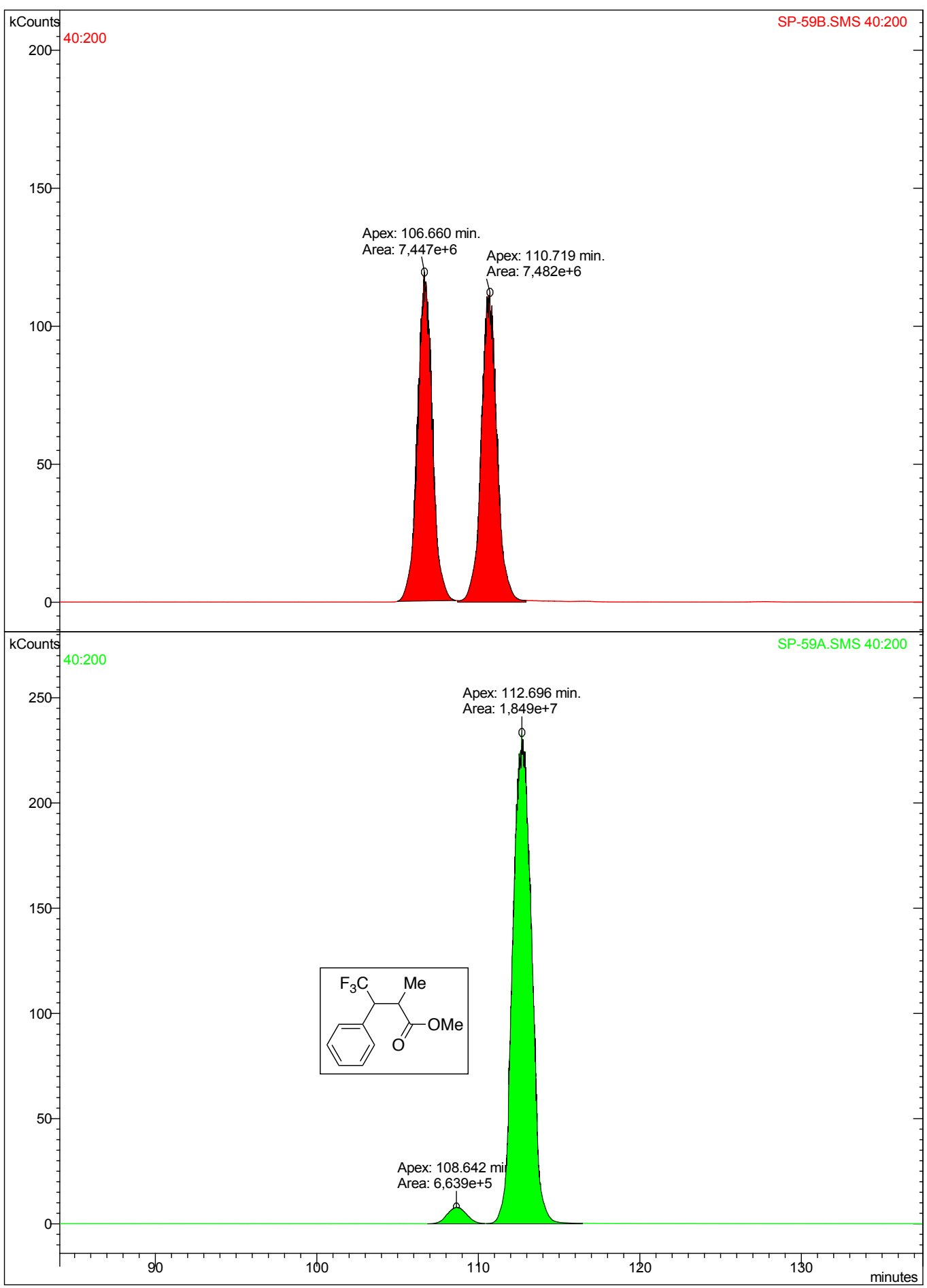



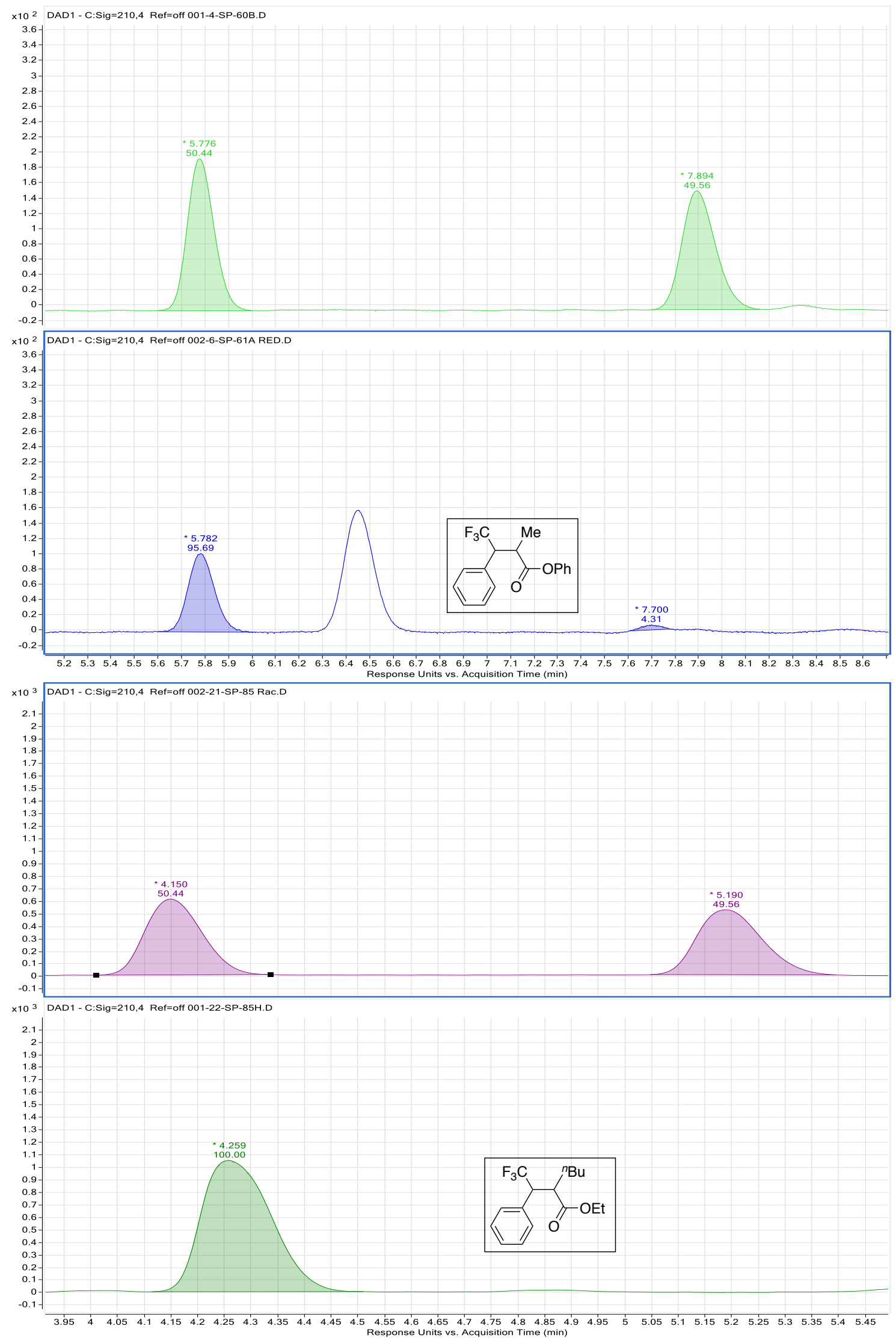
Print Date: 26 Mar 2019 09:35:28

\section{Chromatogram Plots}

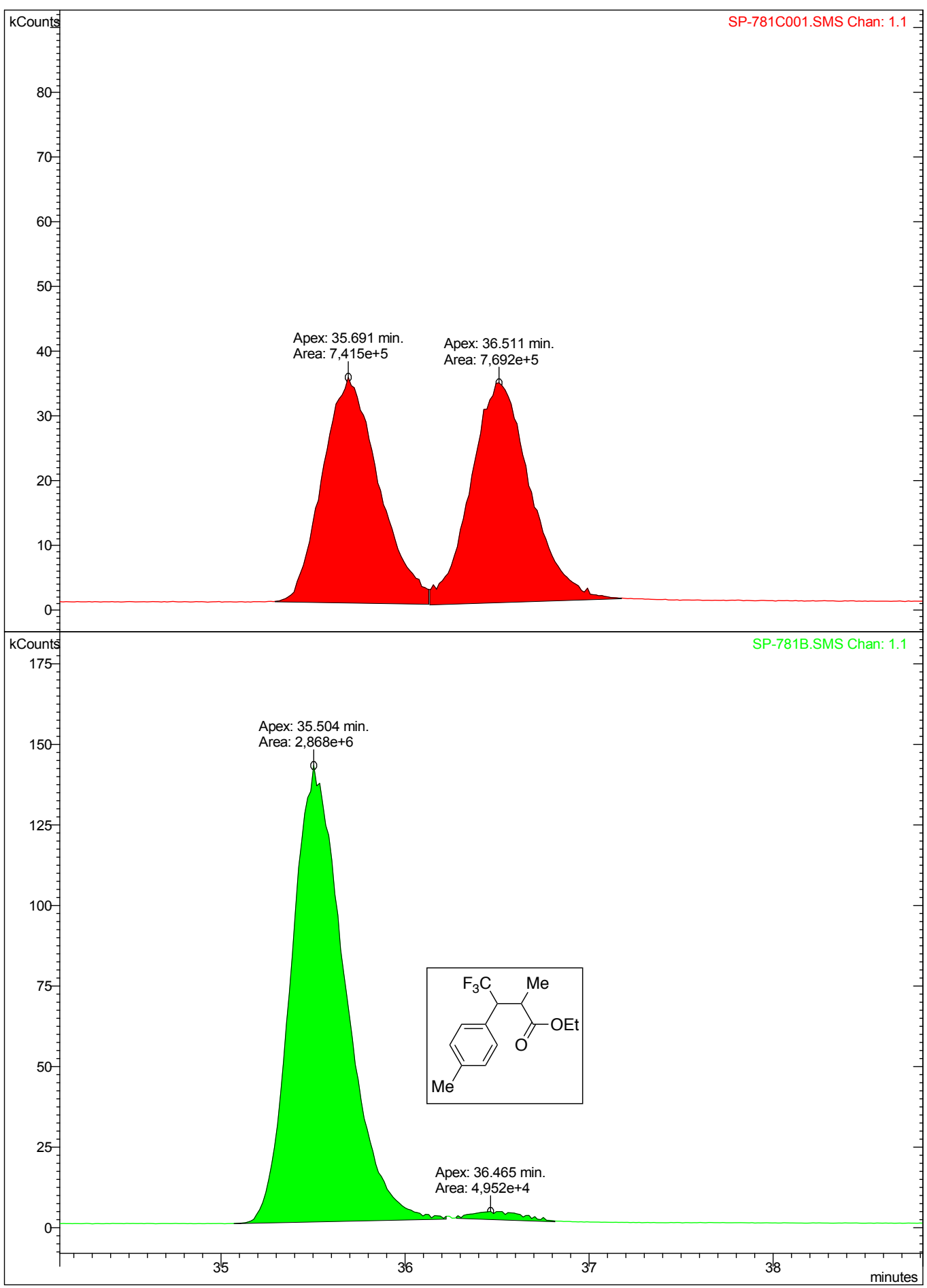


Print Date: 26 Mar 2019 09:24:37

\section{Chromatogram Plots}

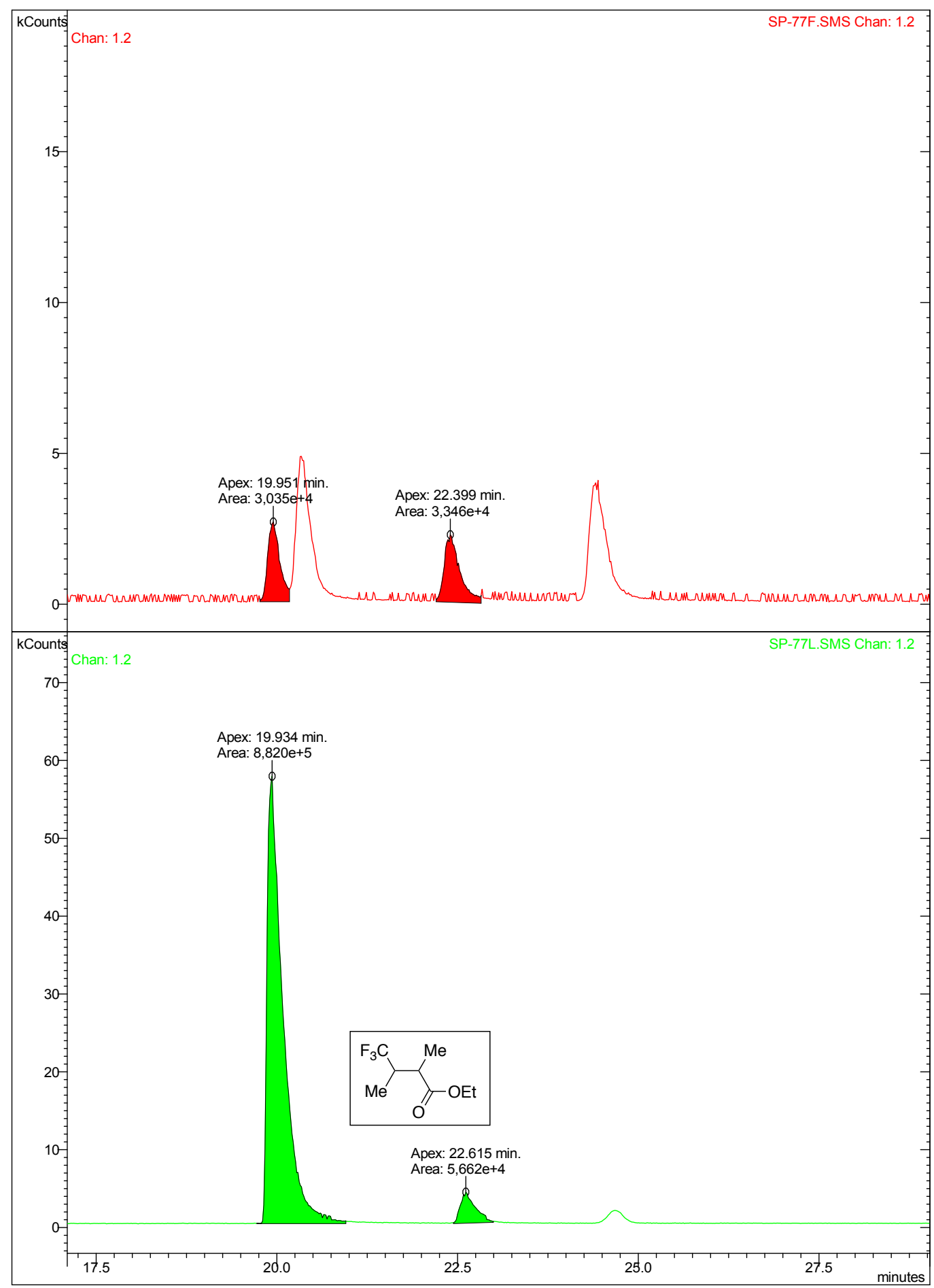


Print Date: 26 Mar 2019 09:31:38

\section{Chromatogram Plots}

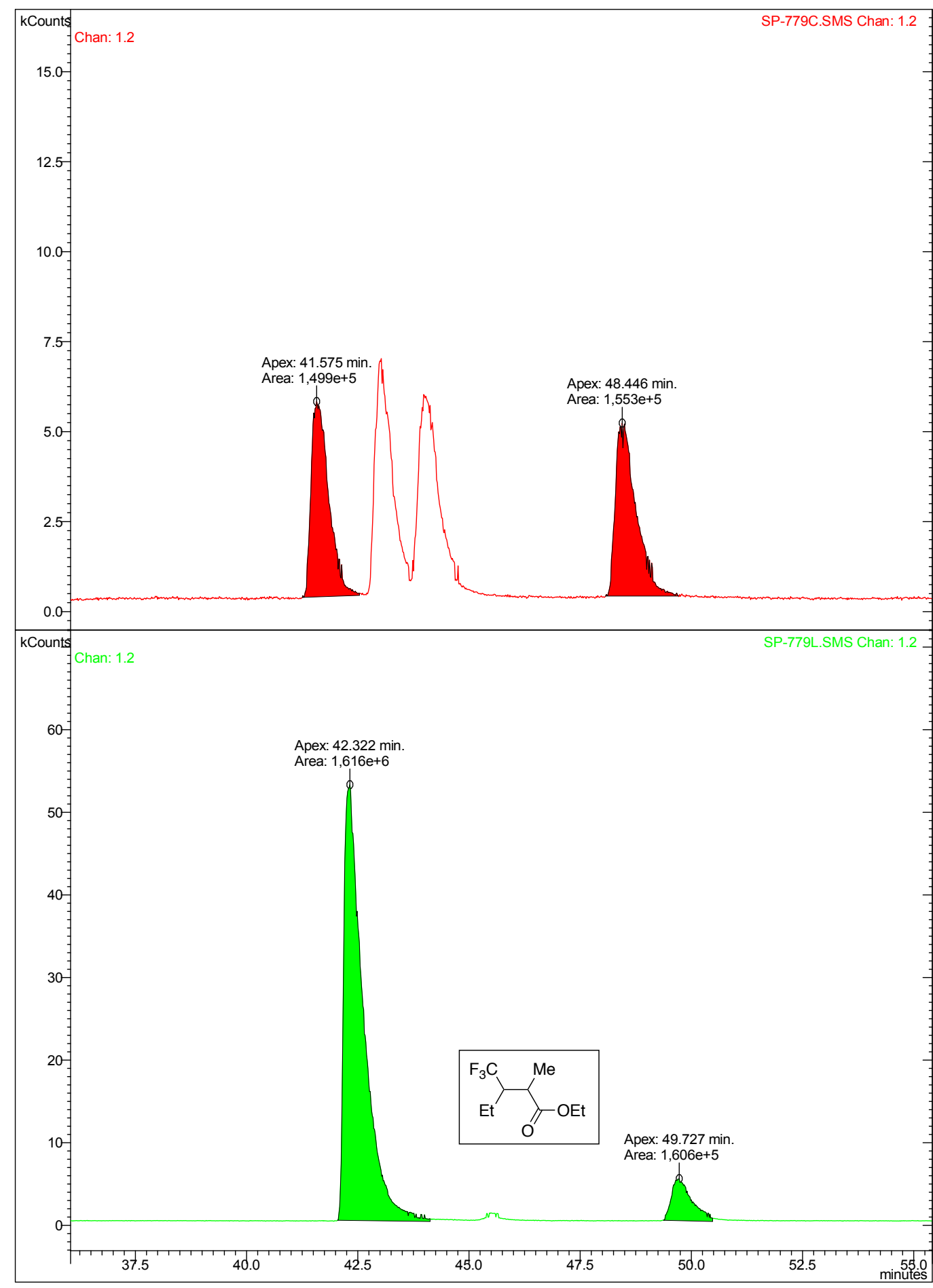



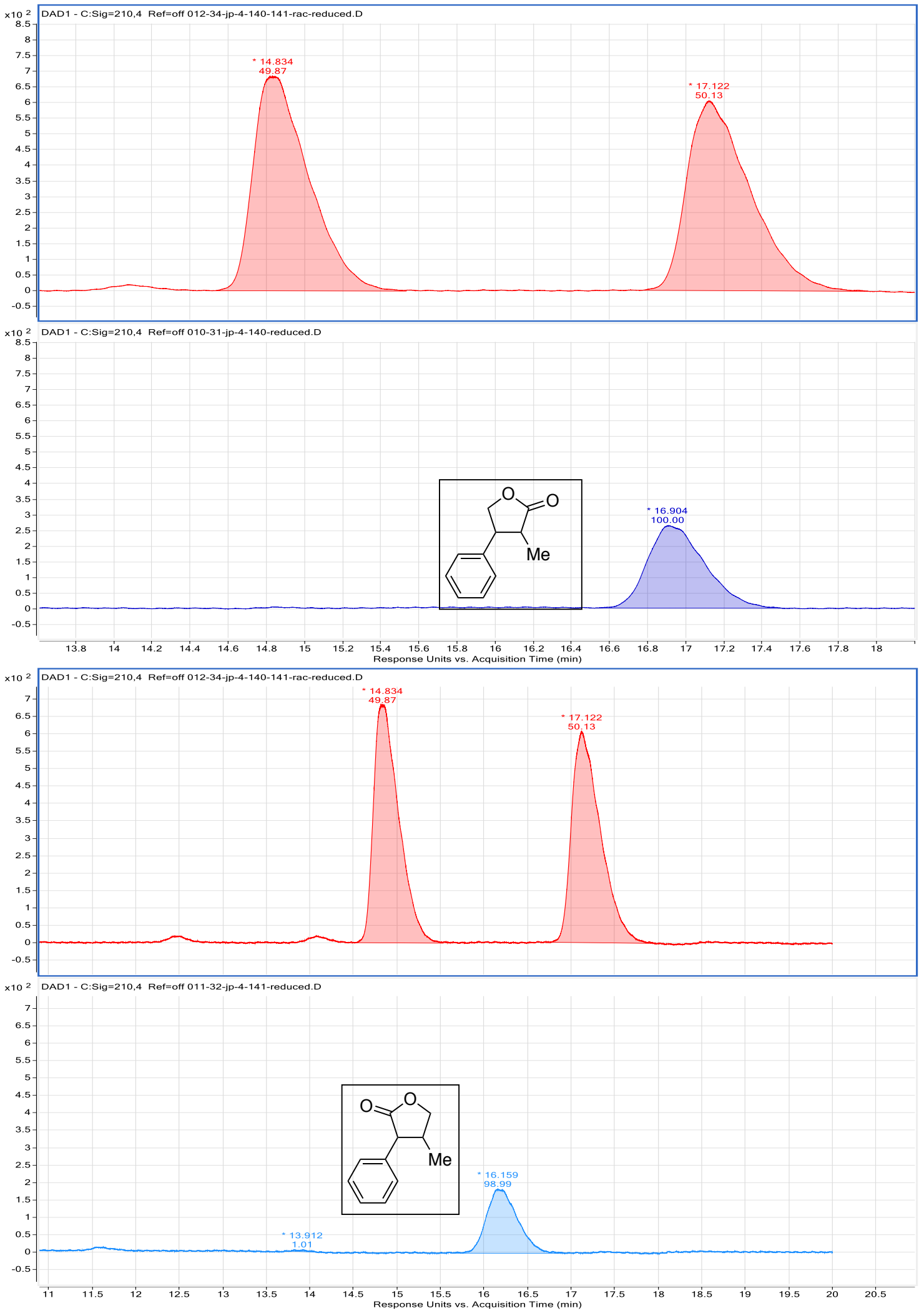

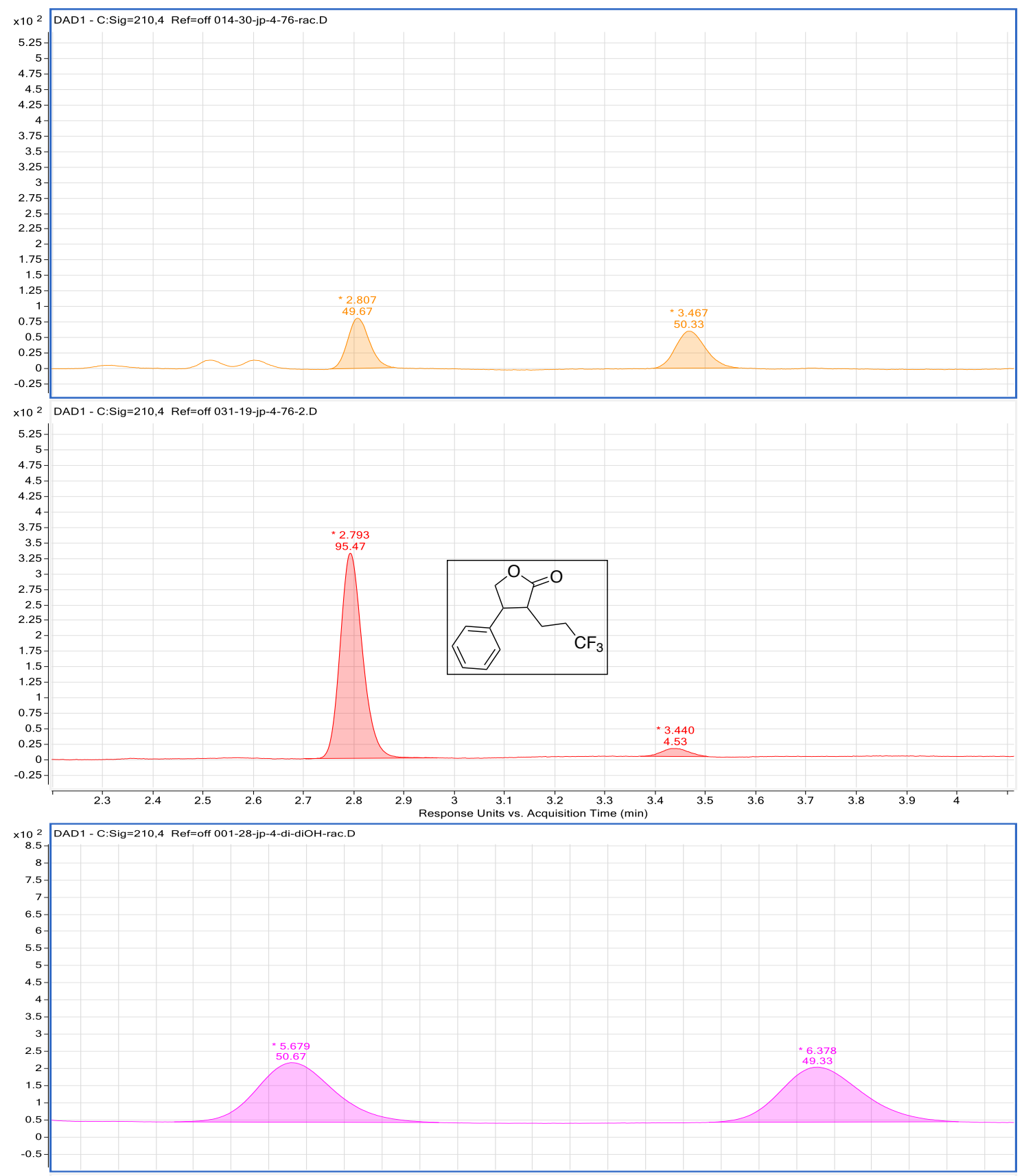

$\times 102$ DAD1 - C:Sig=210,4 Ref=off 003-18-jp-4-di-lactone-reduced.D

$\times 10^{2}$ DAD1 - C:Sig=210,4 Ref=off 003-18-jp-4-di-
8.5
8 $\quad \begin{gathered}5.656 \\ 97.69\end{gathered}$

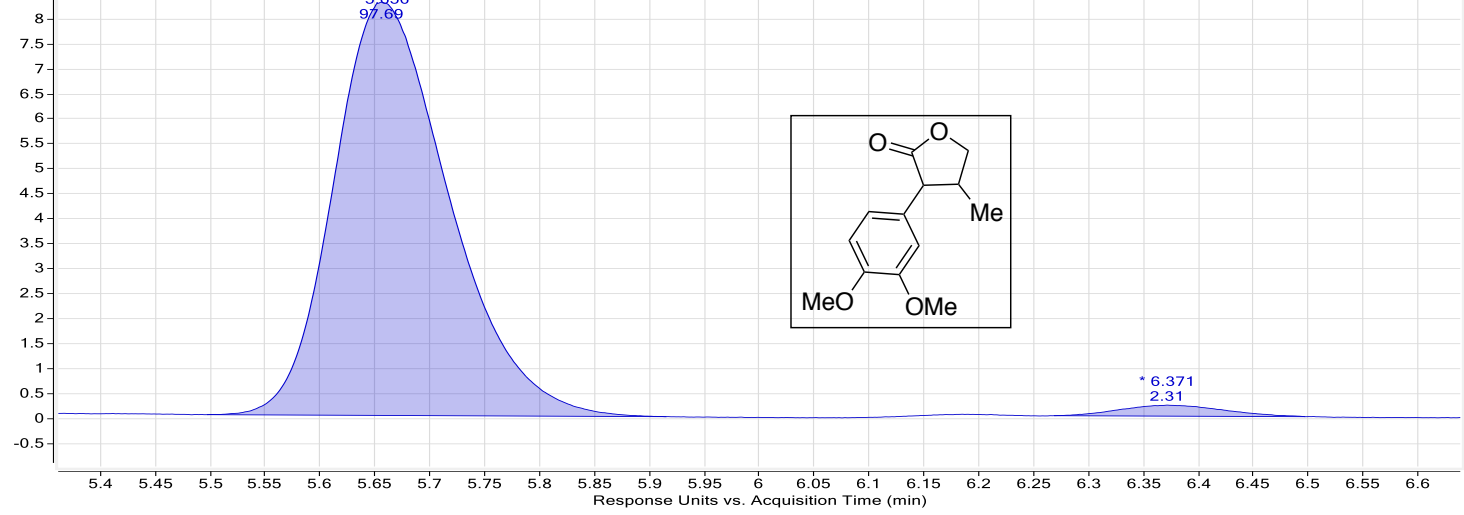




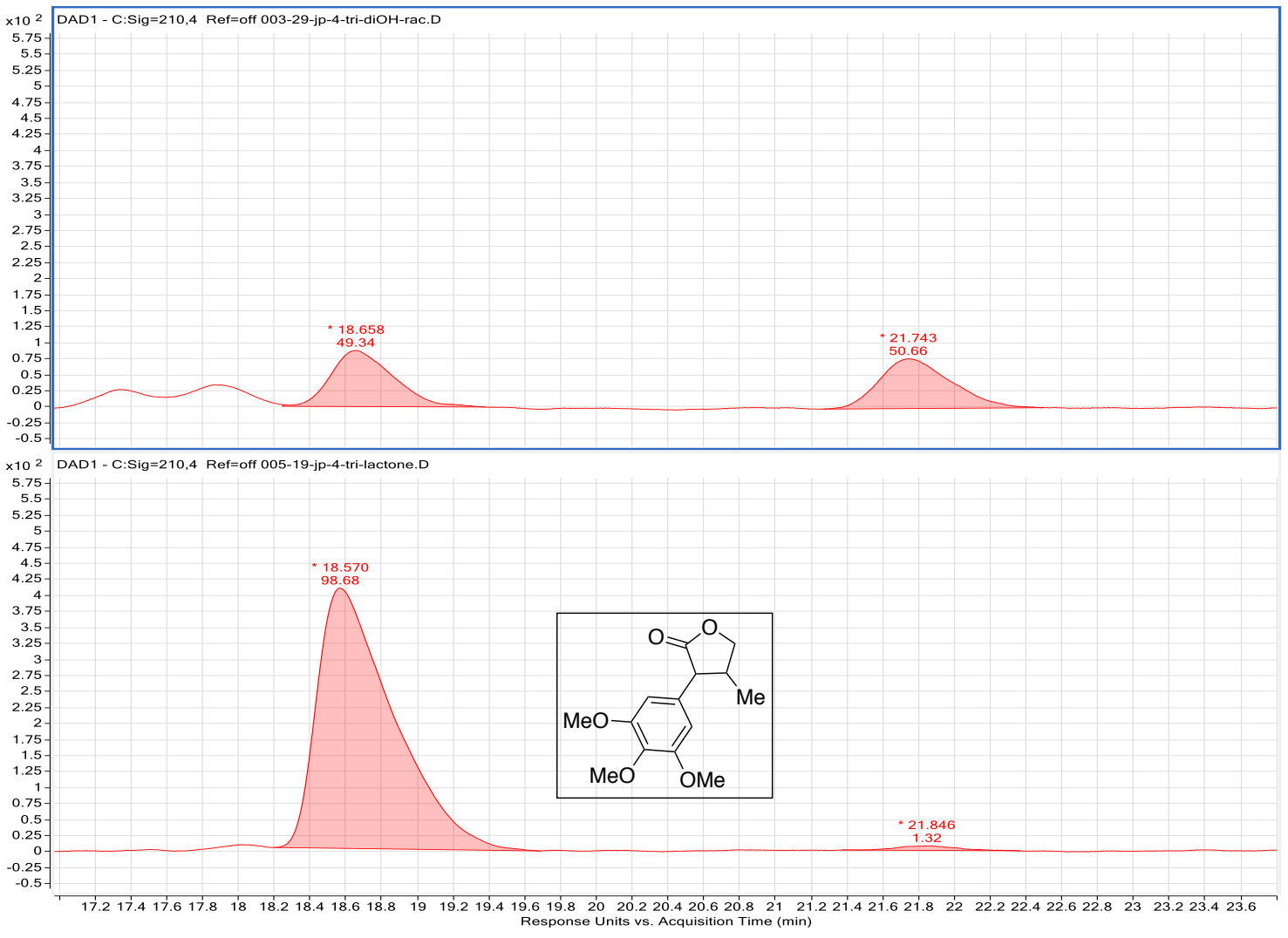

\section{NMR spectrum of the new compounds}

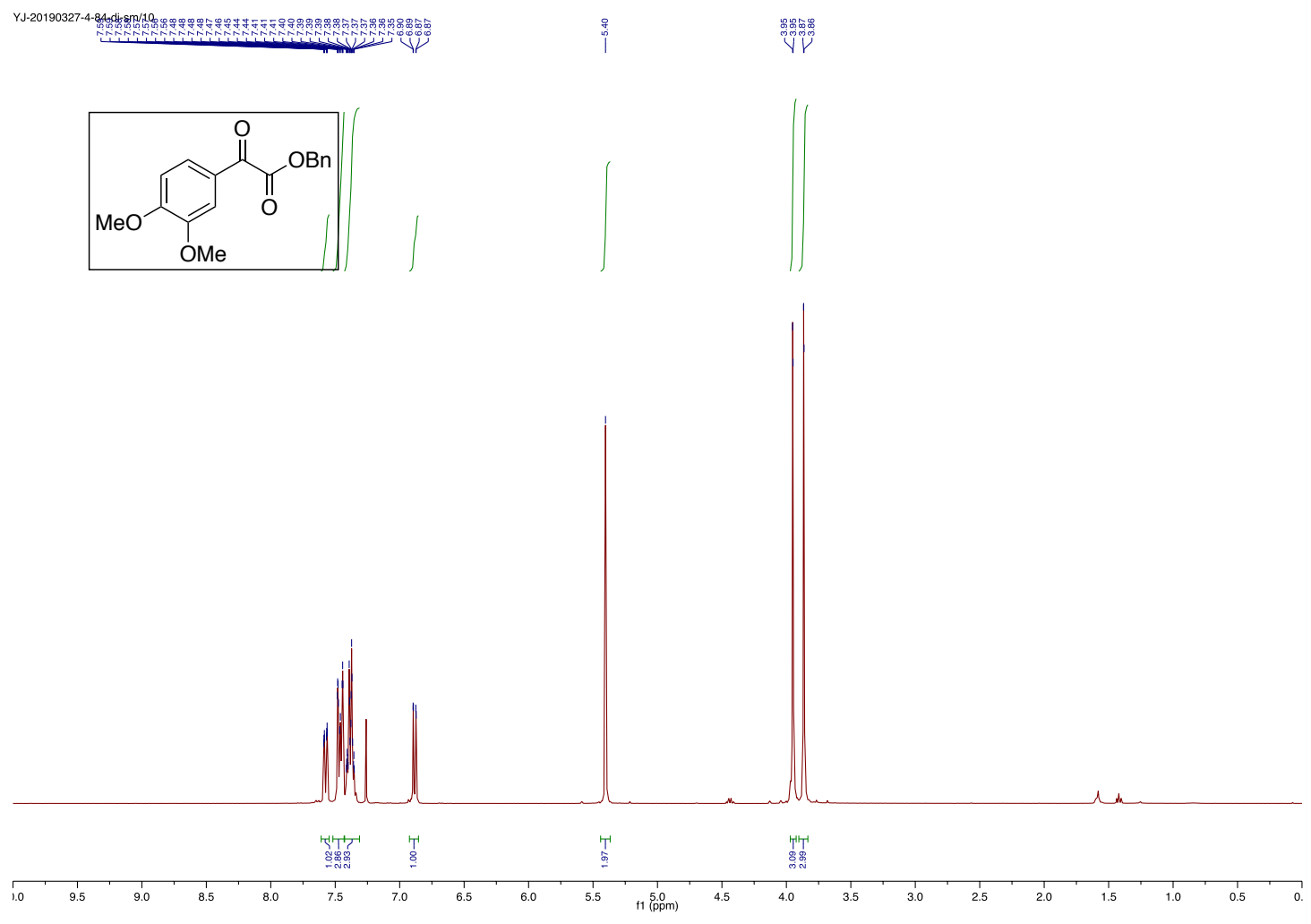



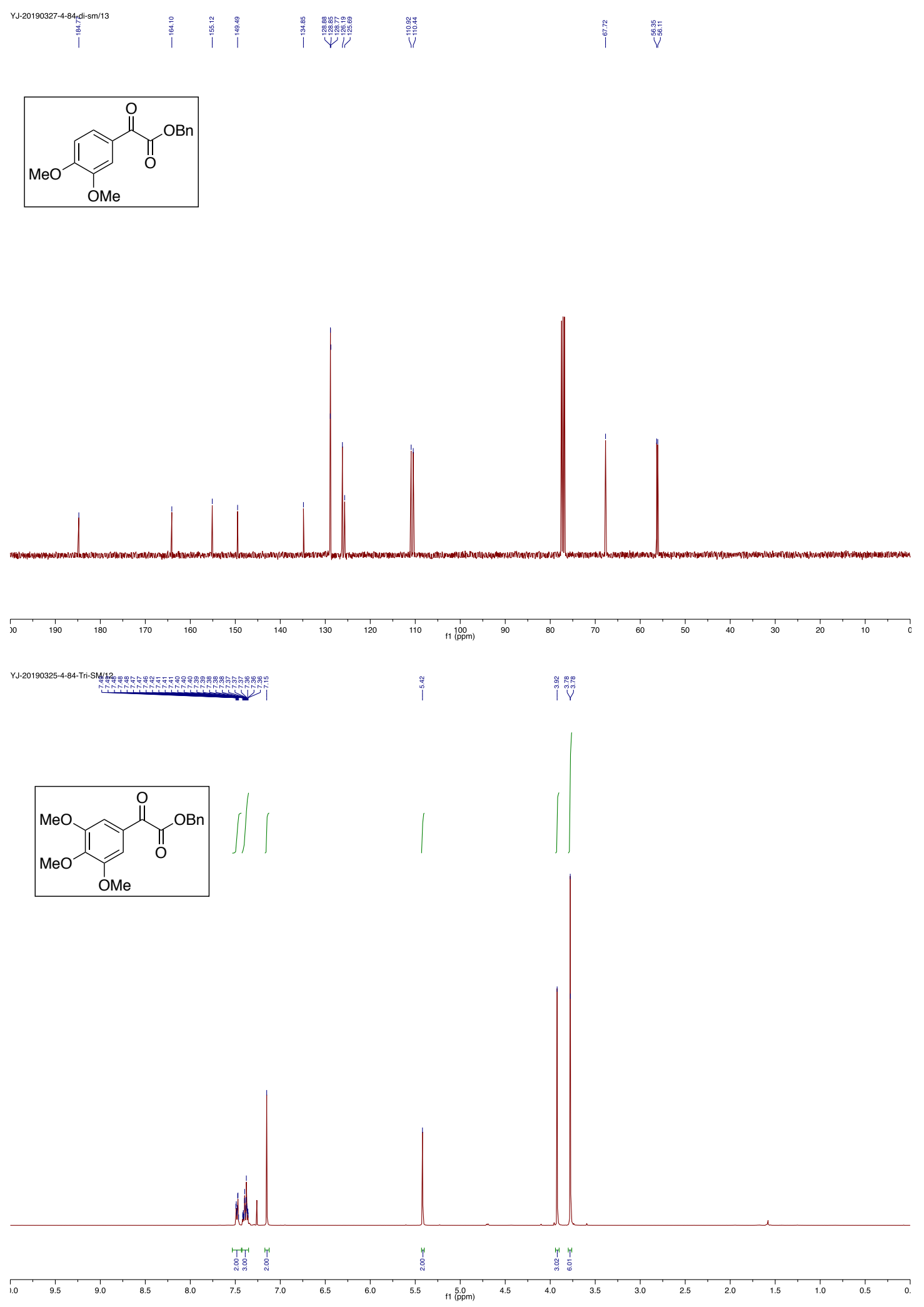

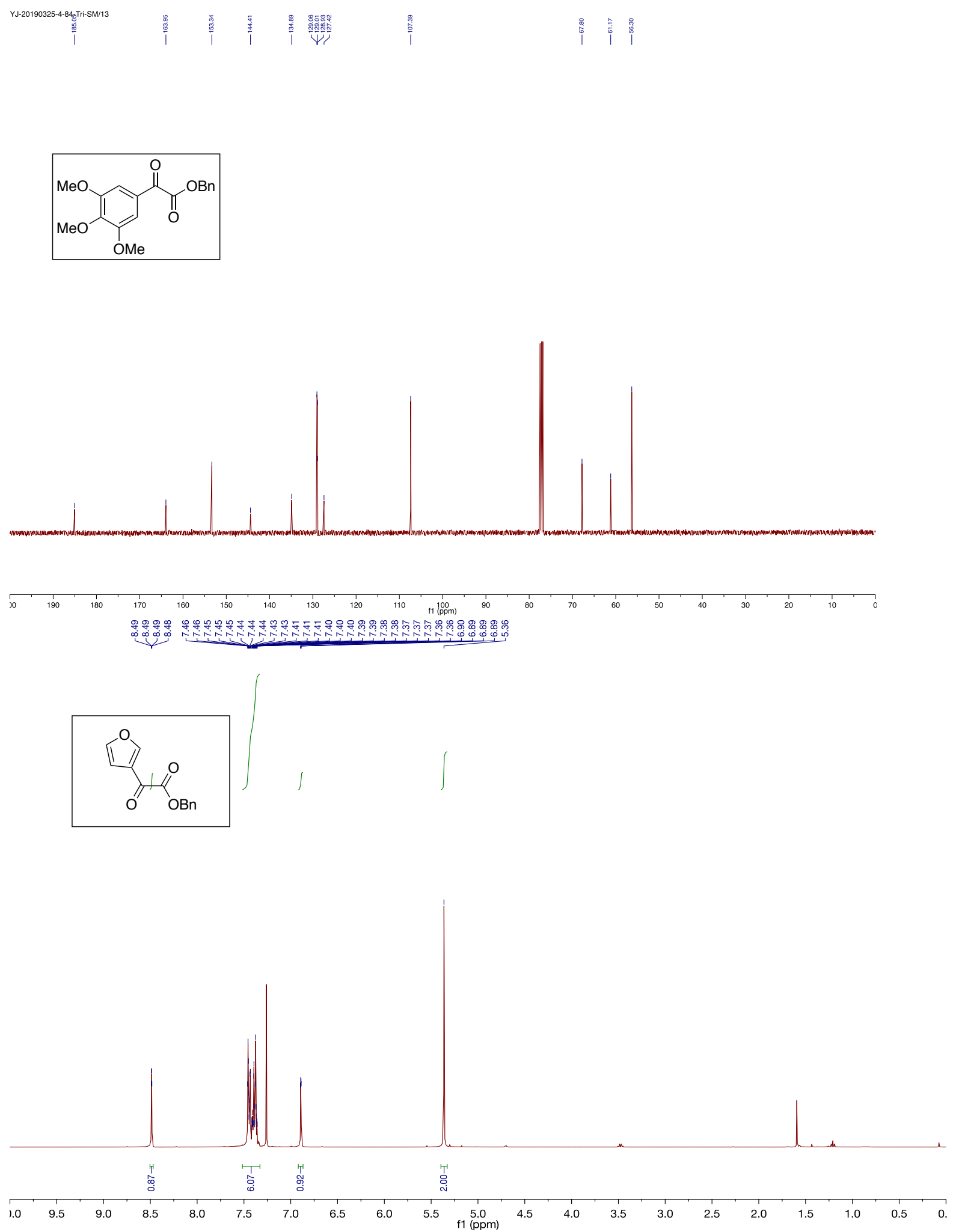

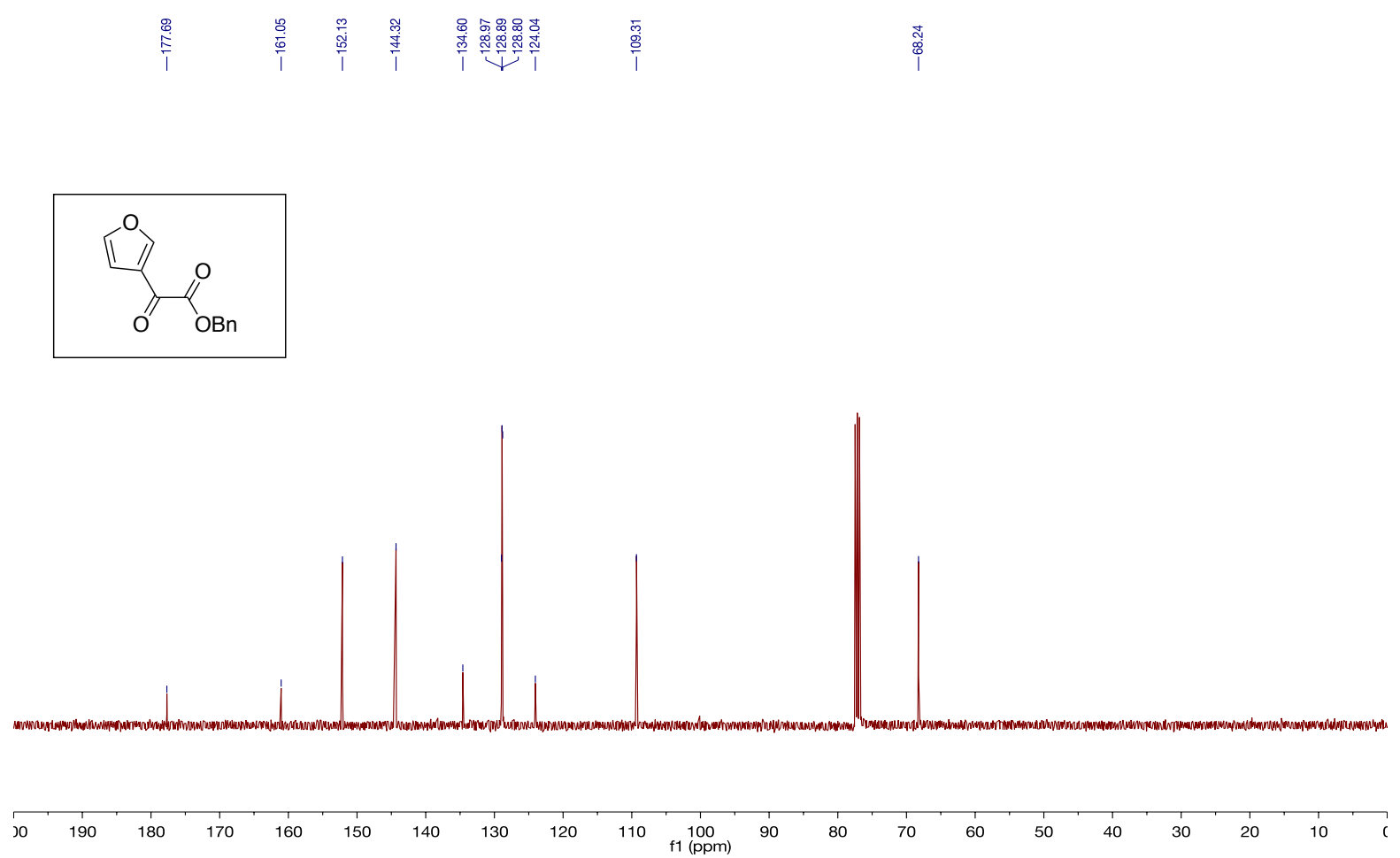

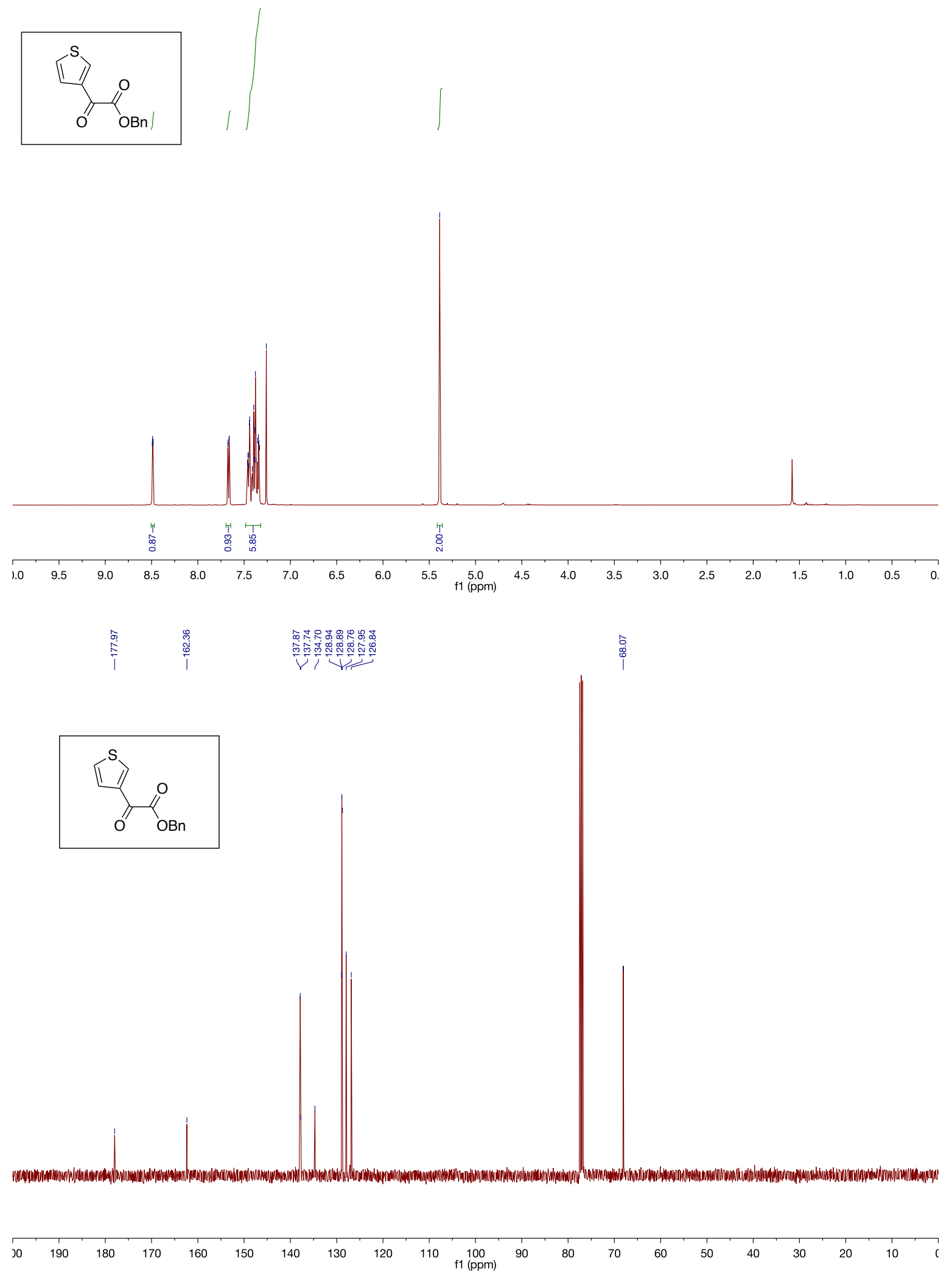

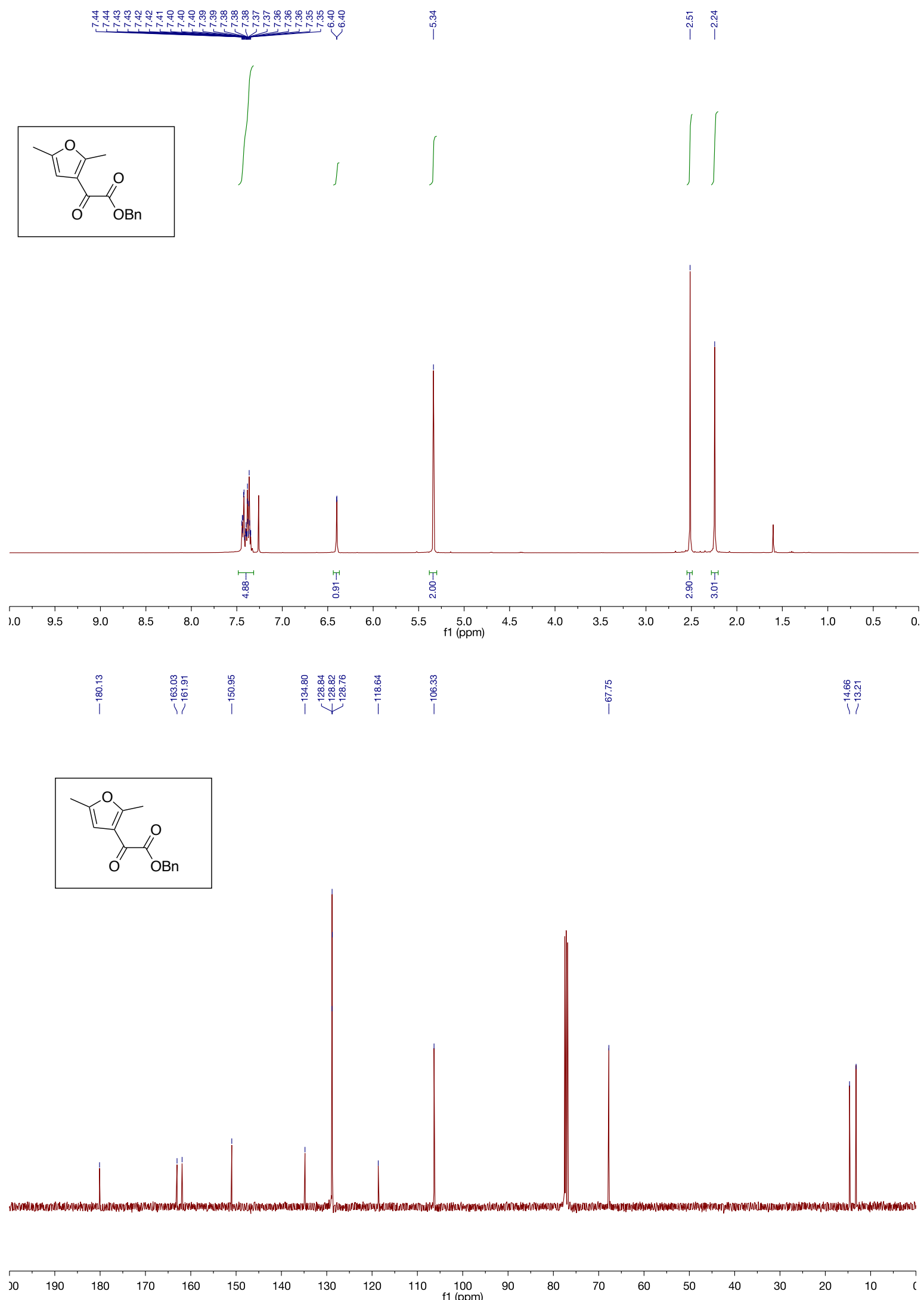

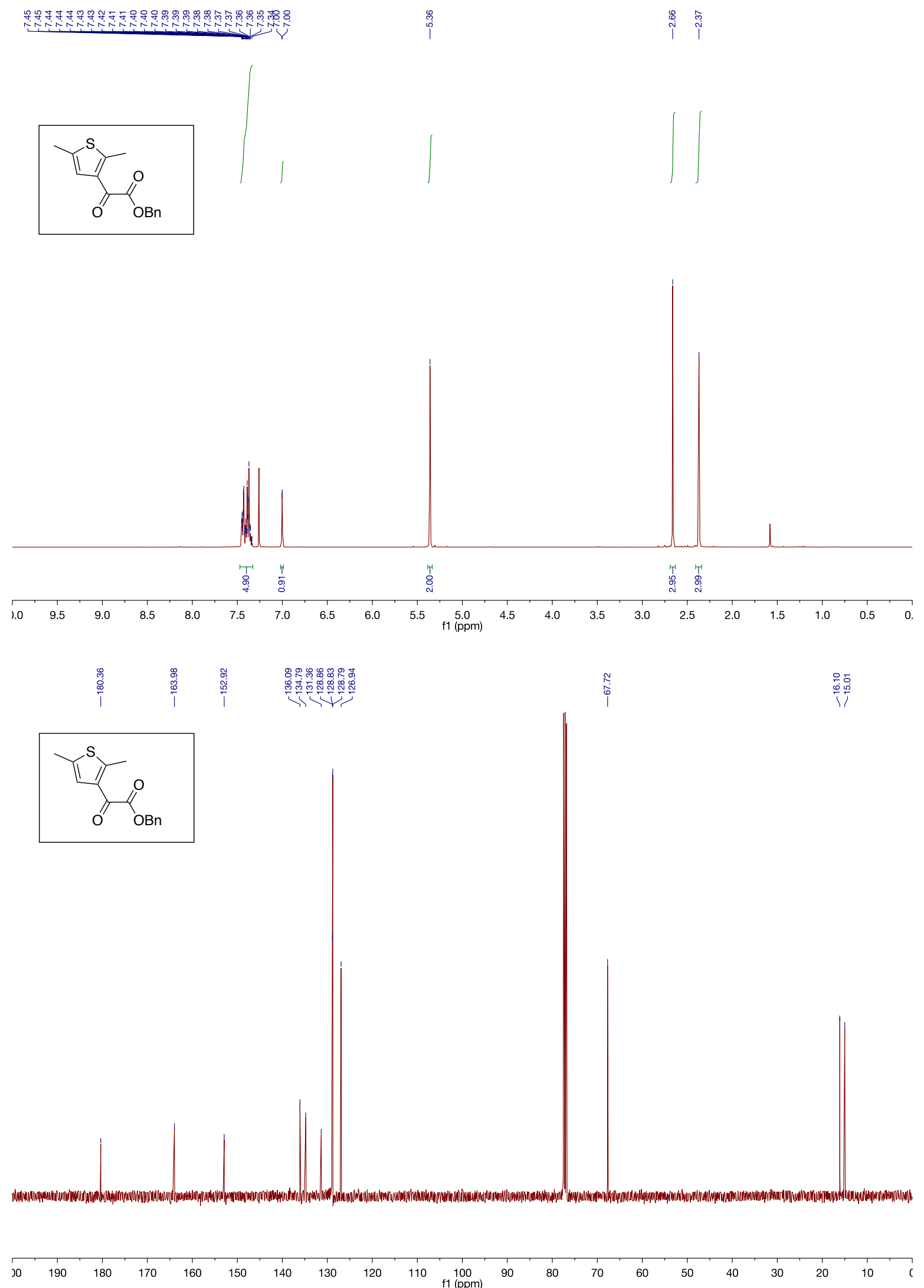

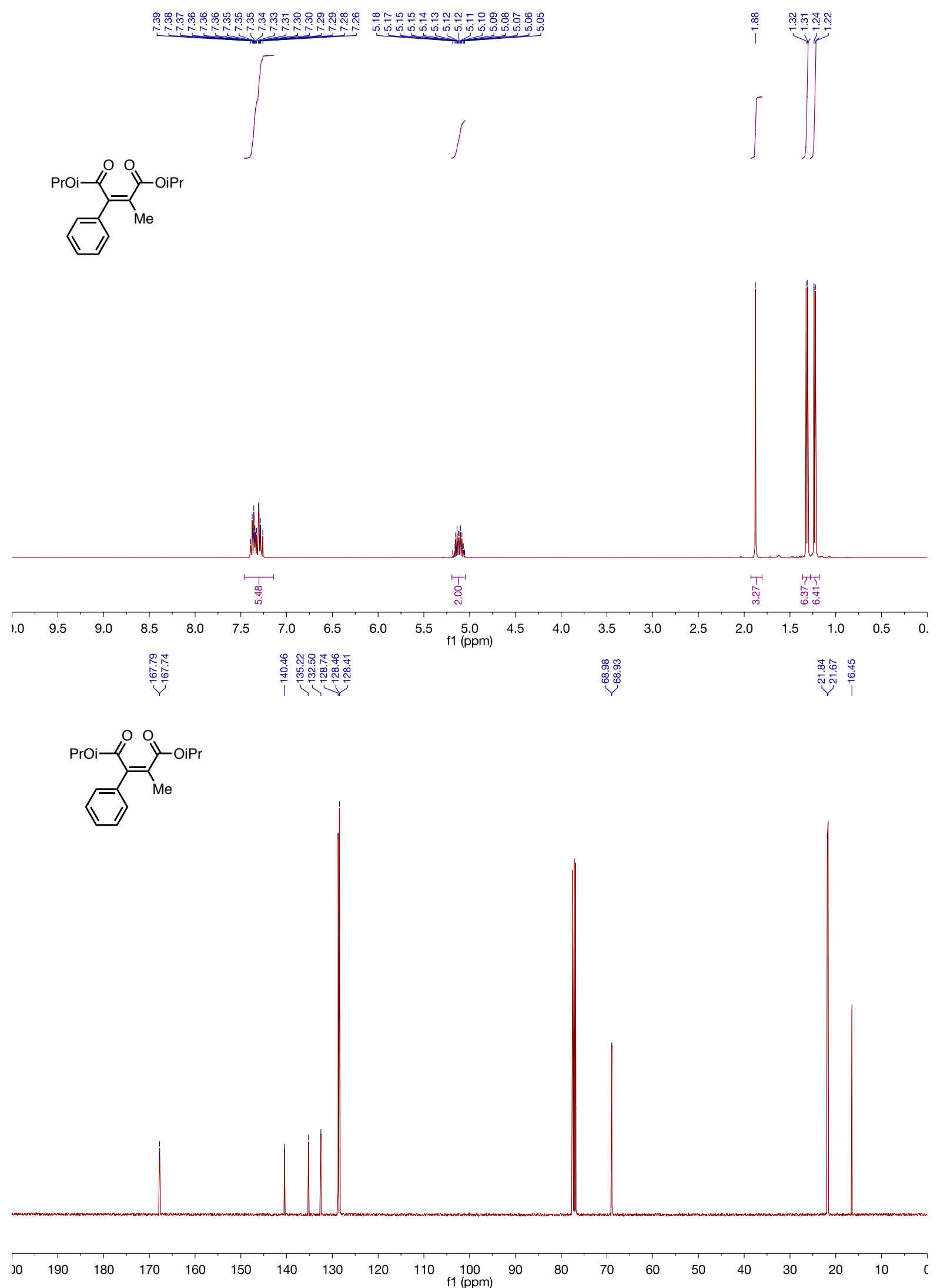

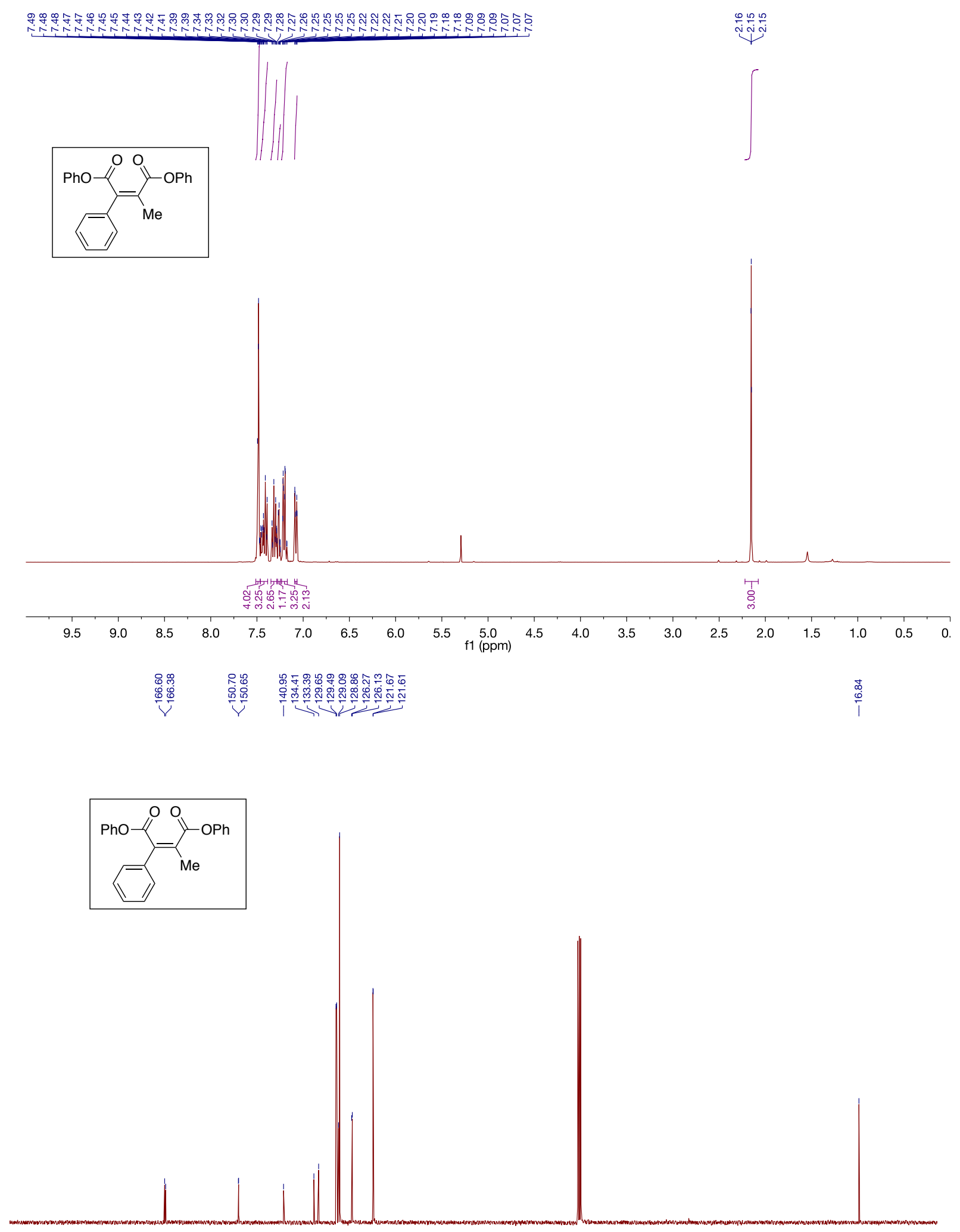

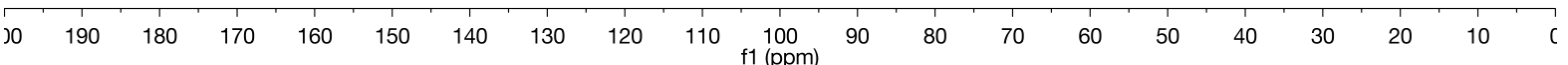



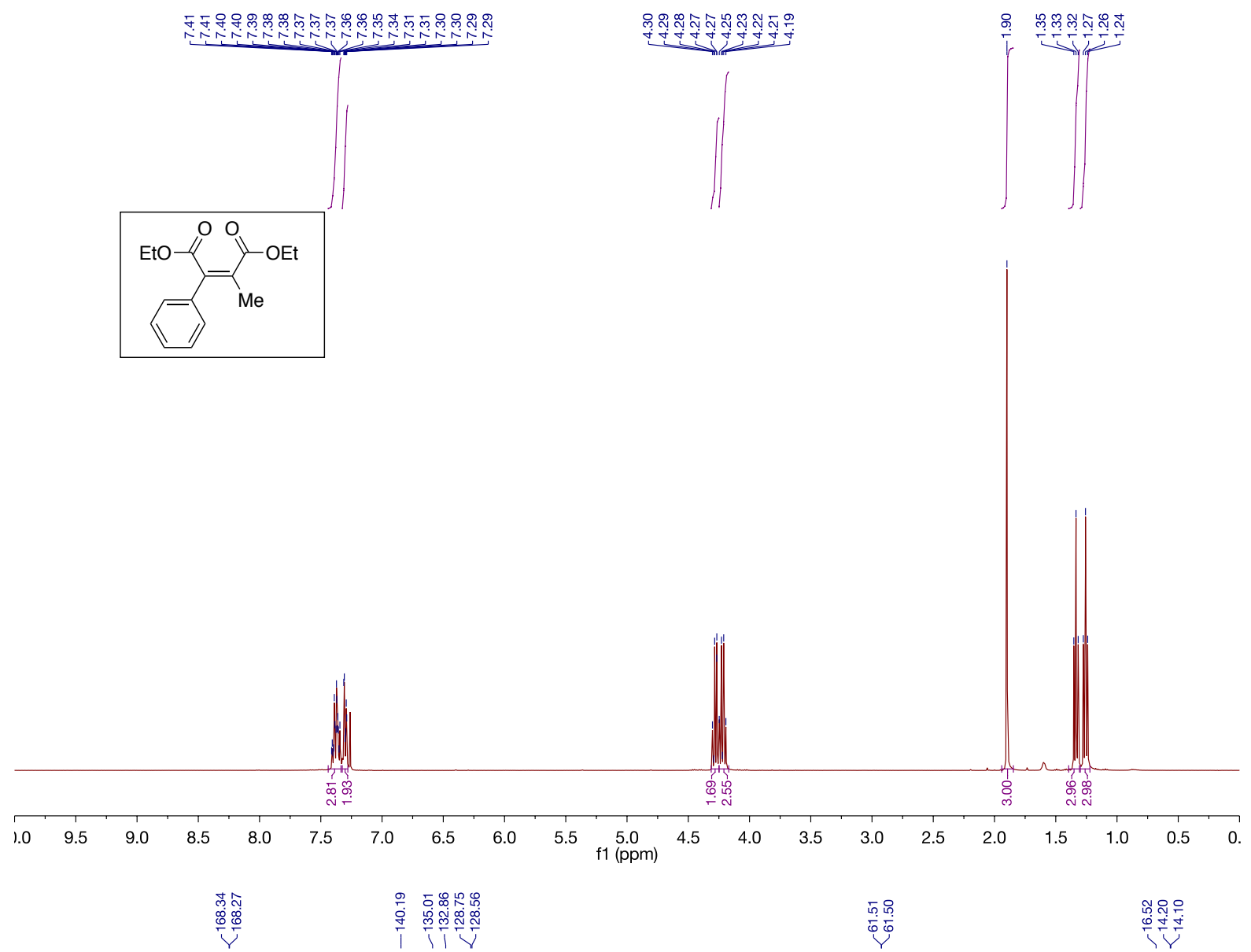

뚱ํํำ

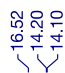
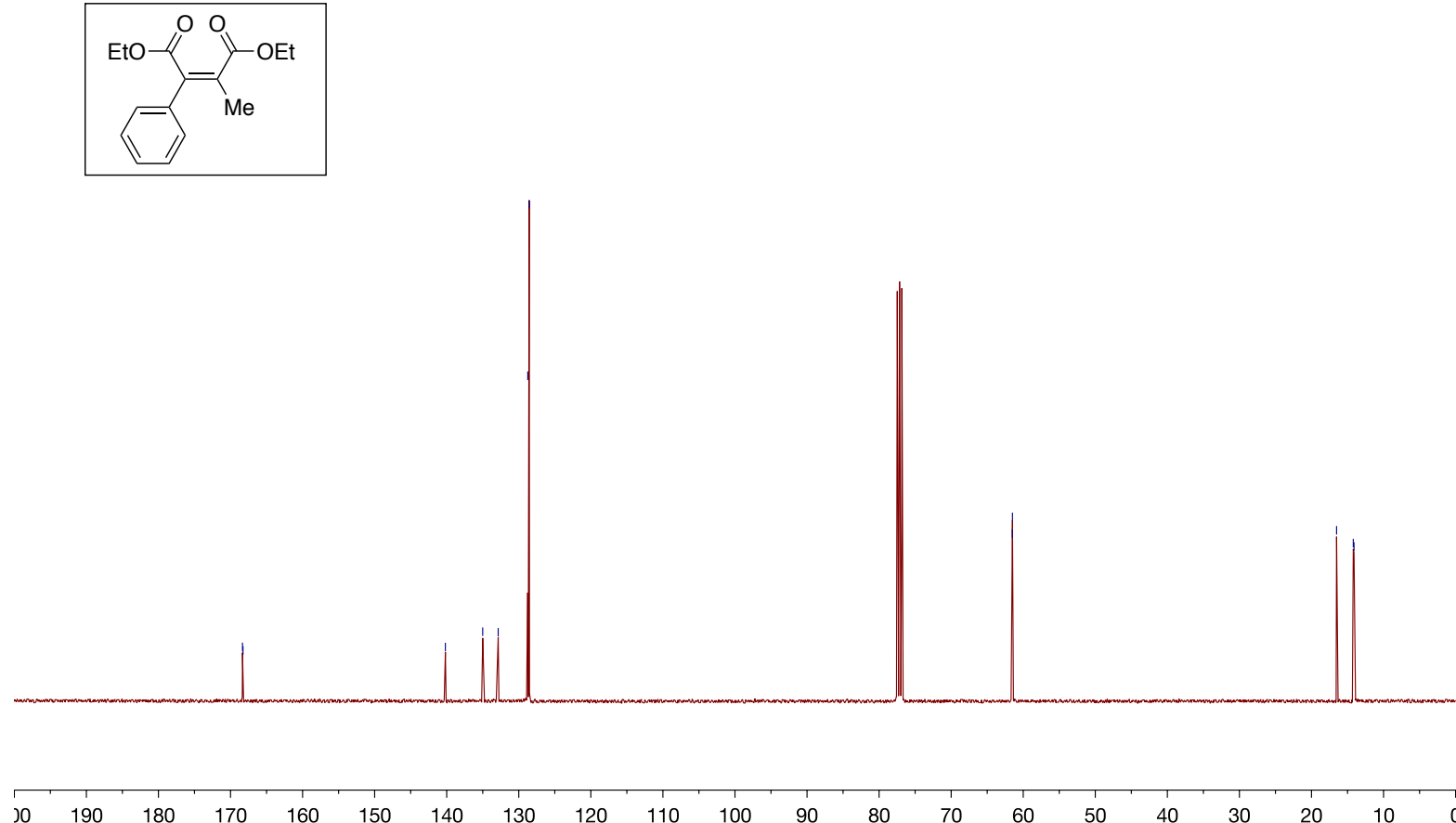

$110 \quad 100 \quad 90$

80

(ppm)

S-73 


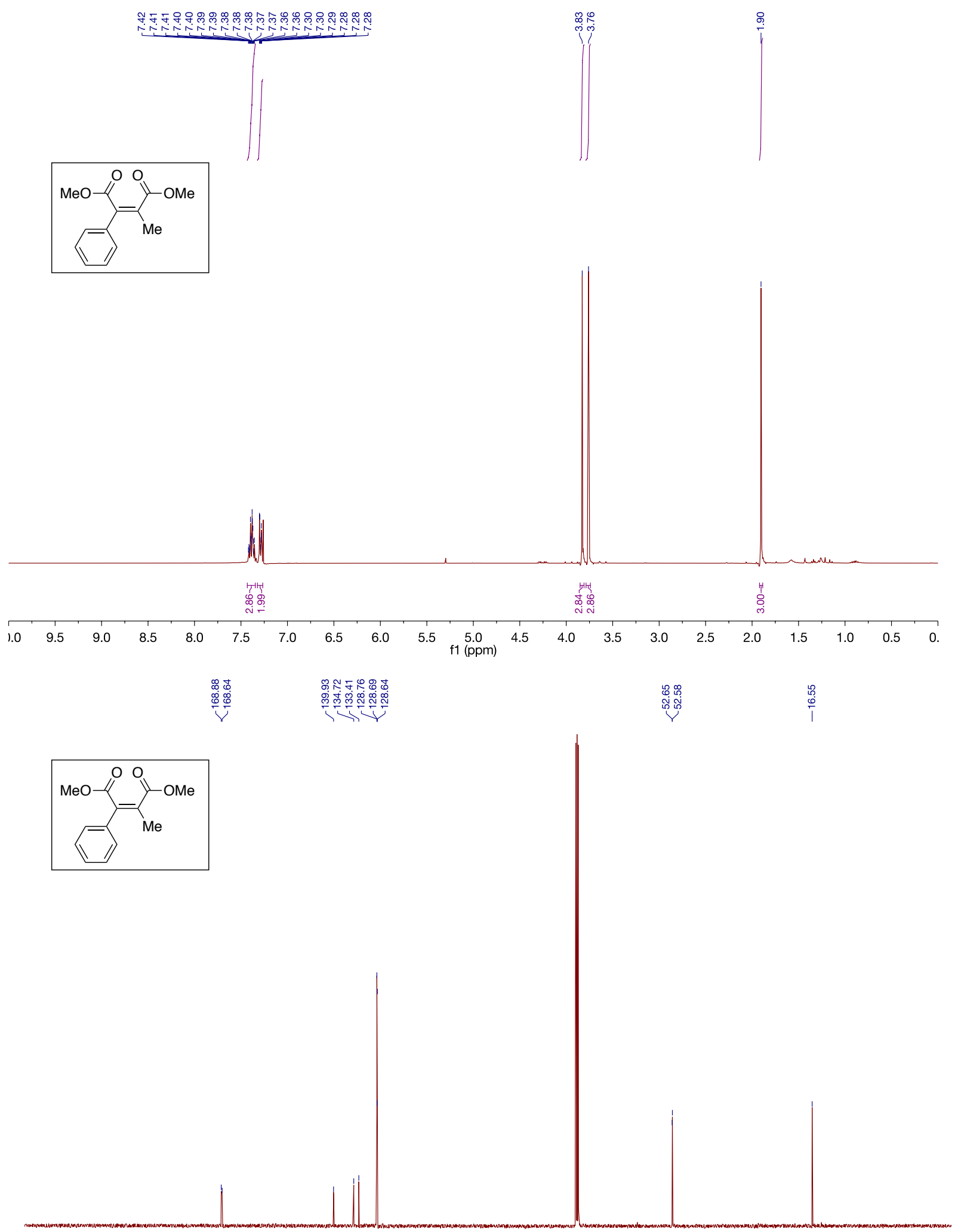

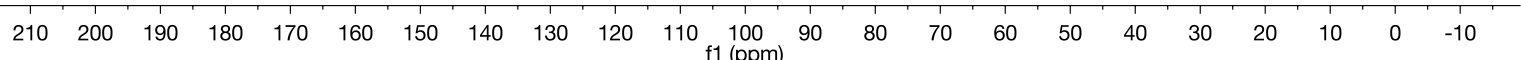



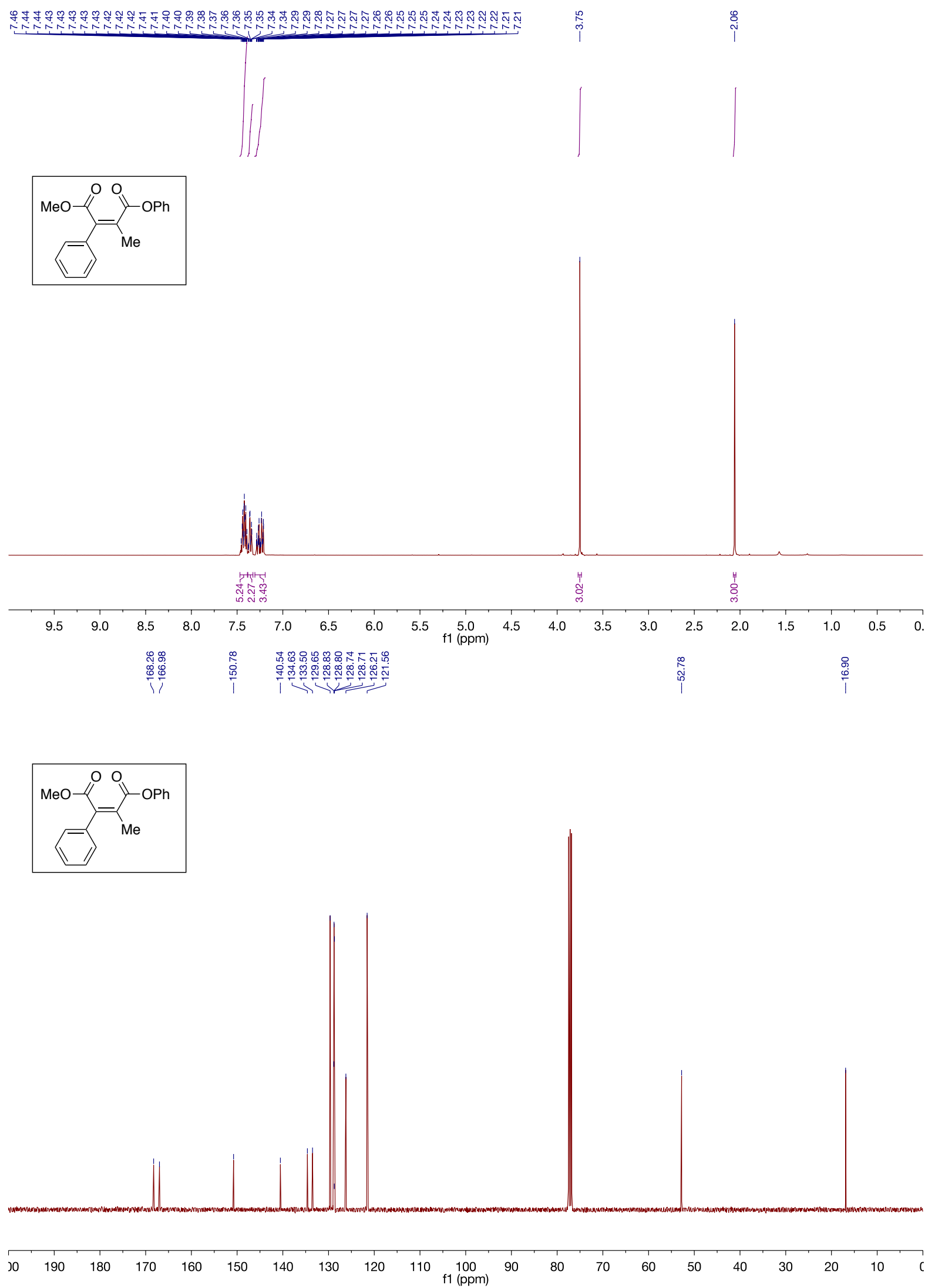

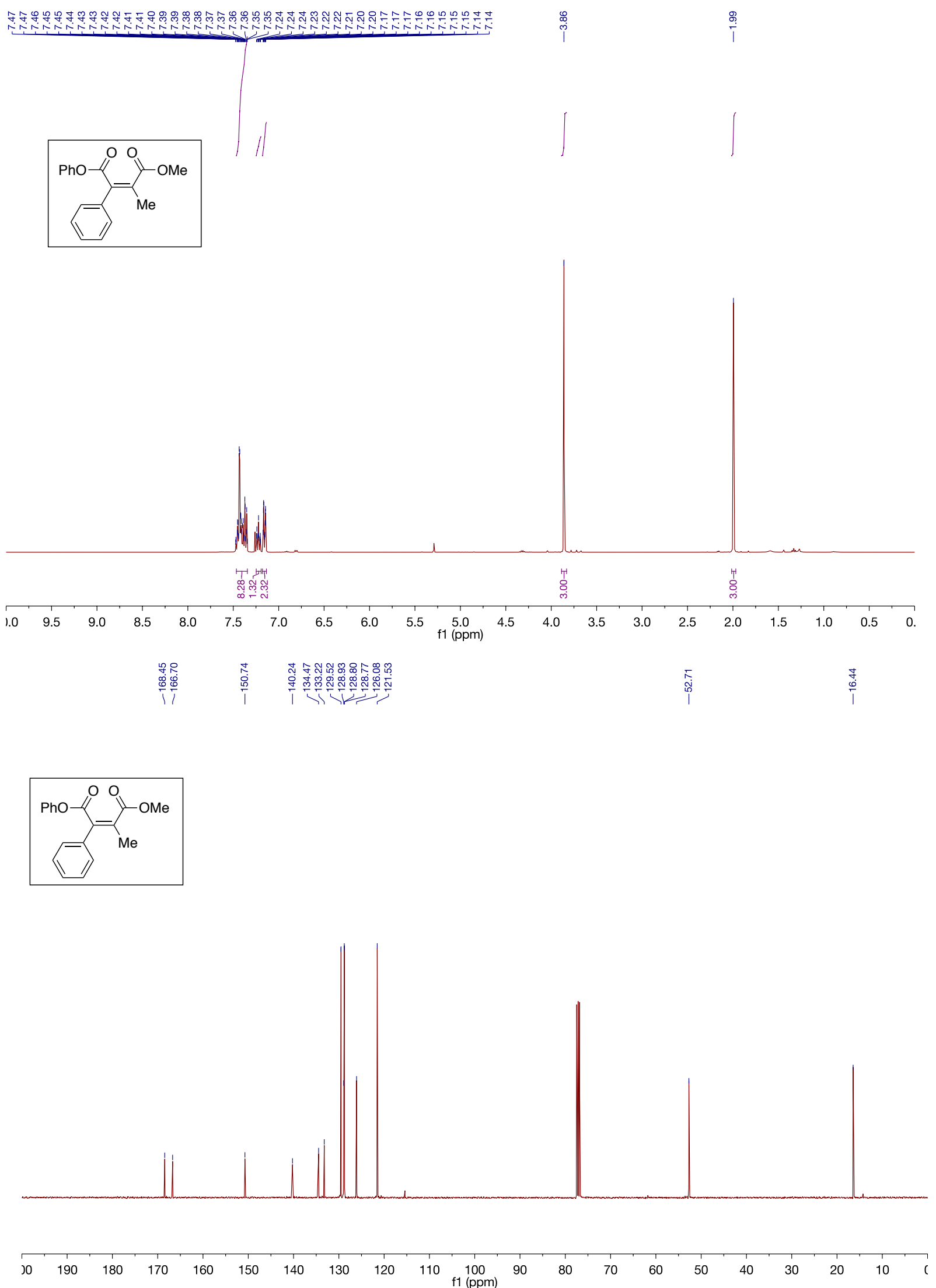

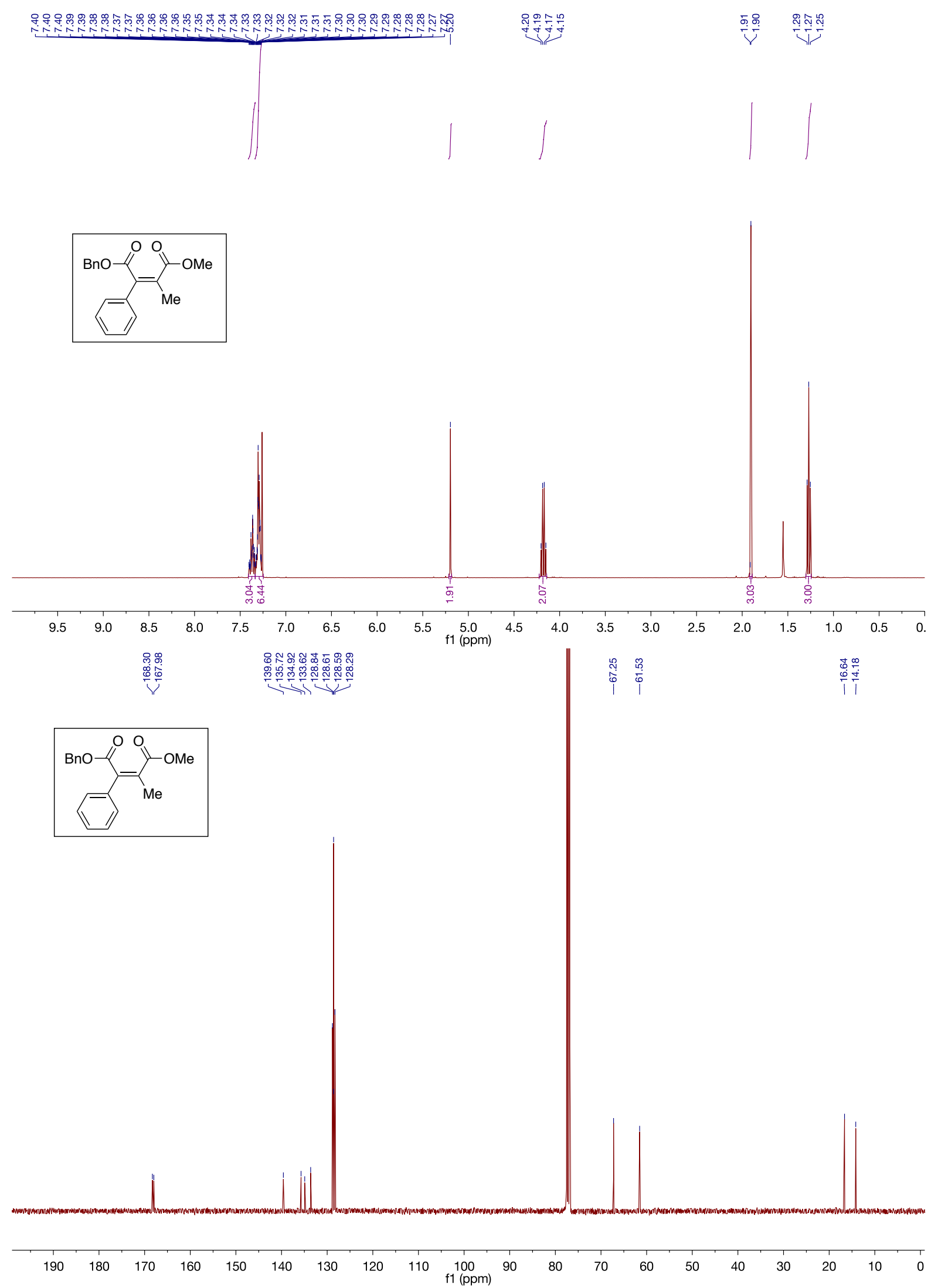

S-77 
jp-190307-4-129-hplc/10
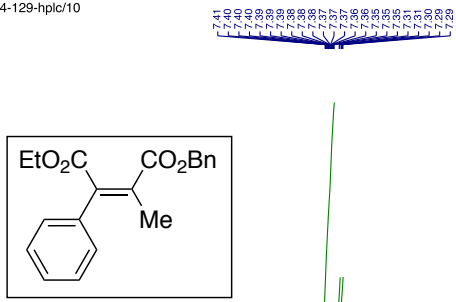

i̊

ำ

İ

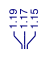
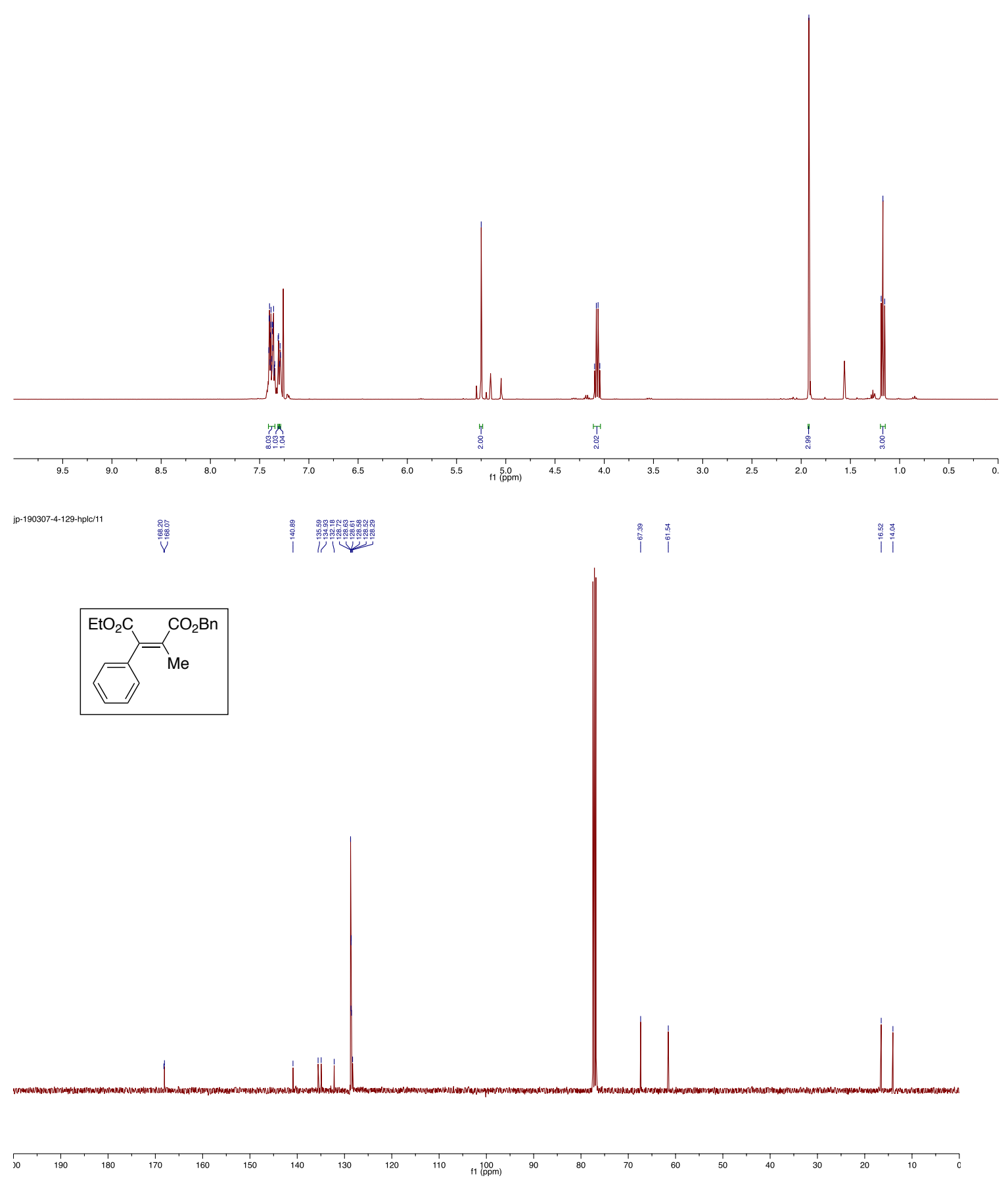

S-78 


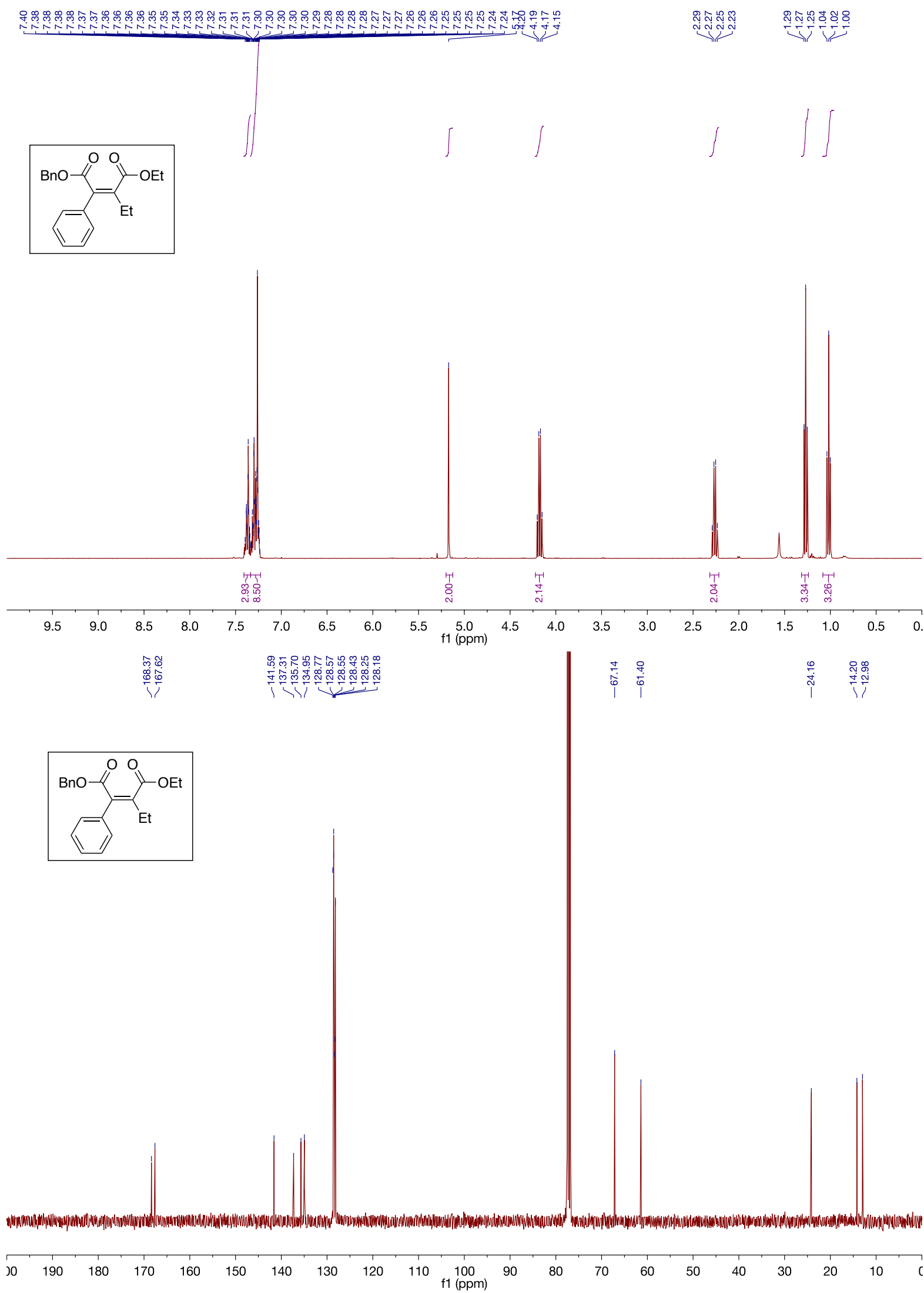




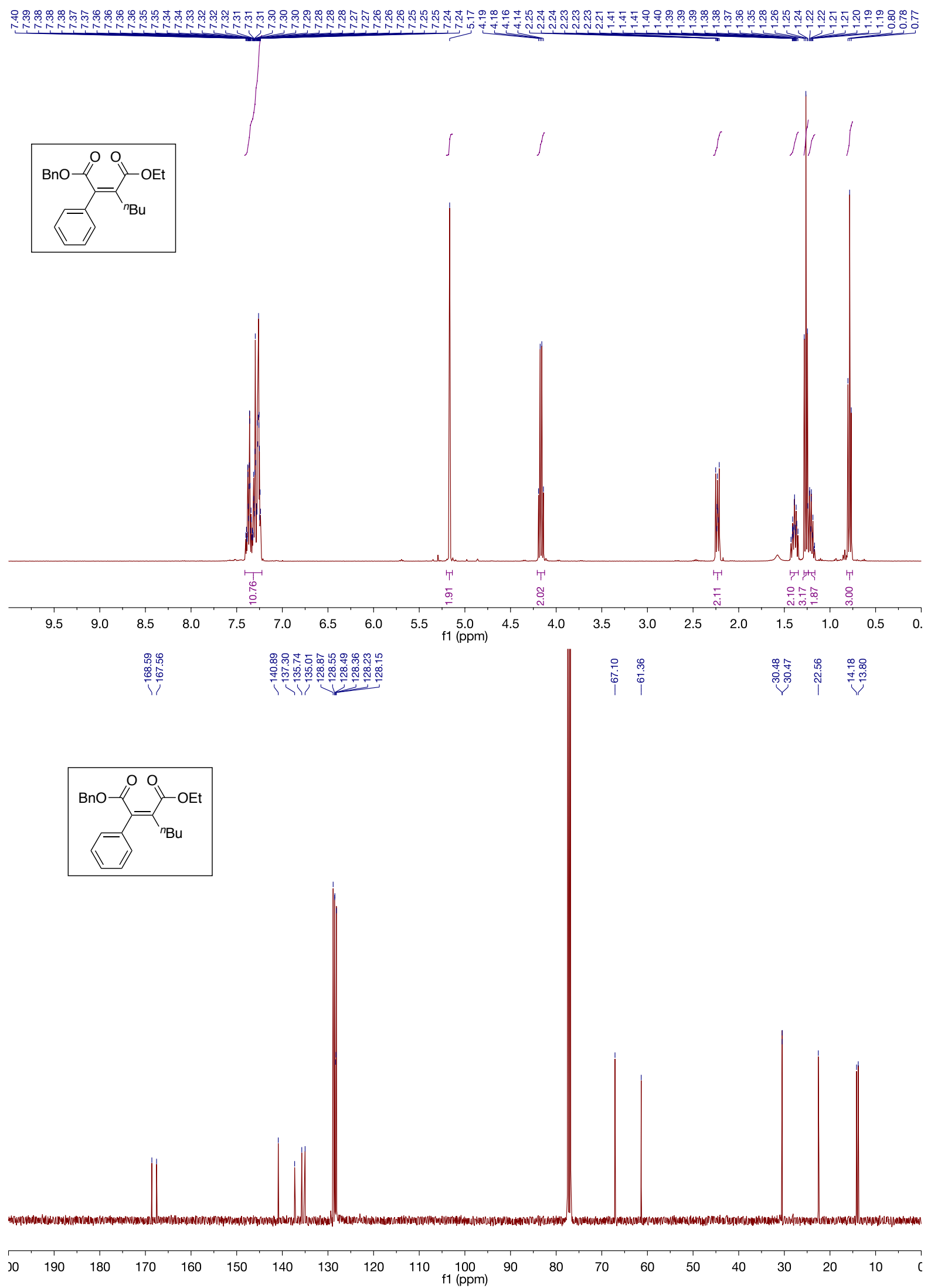



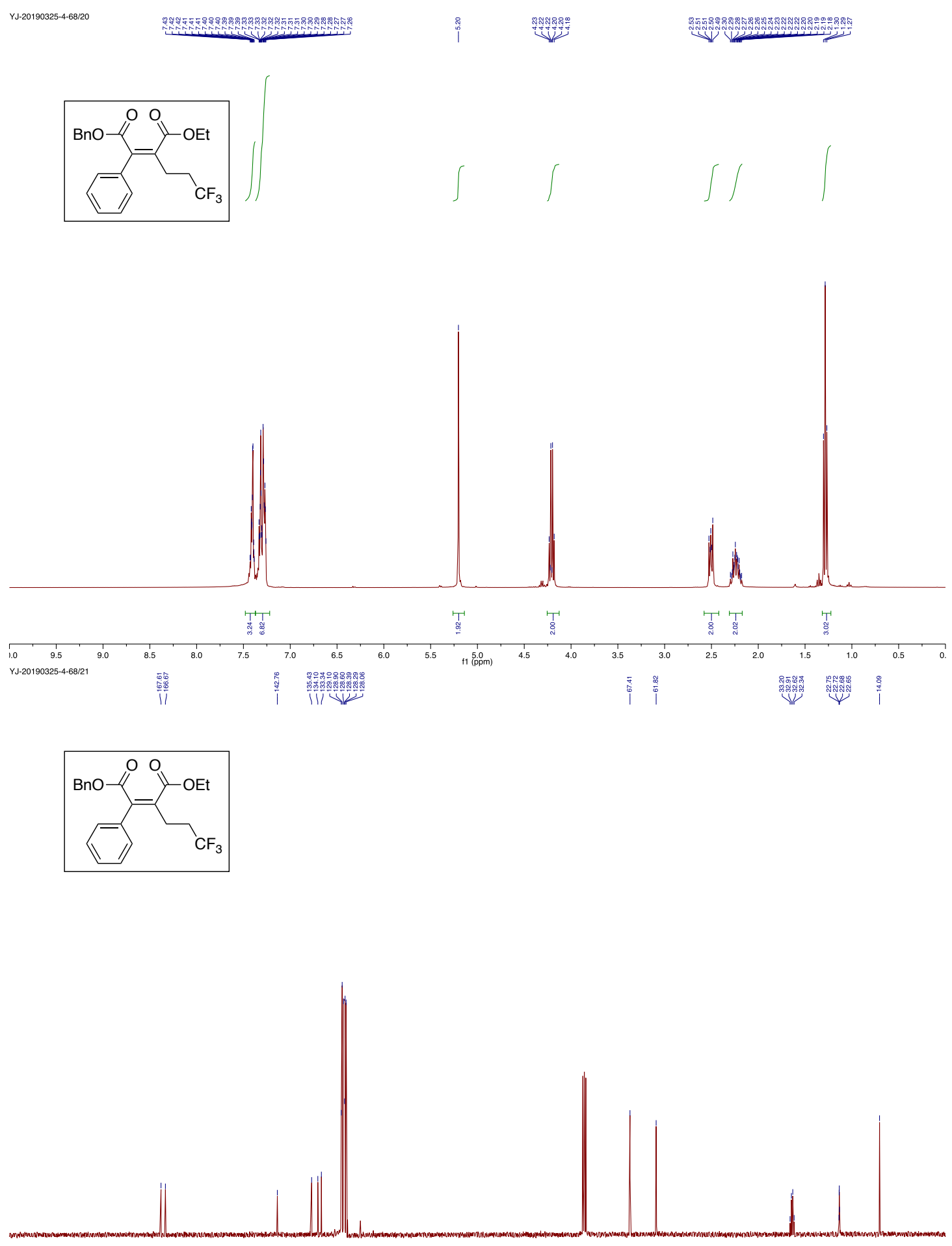

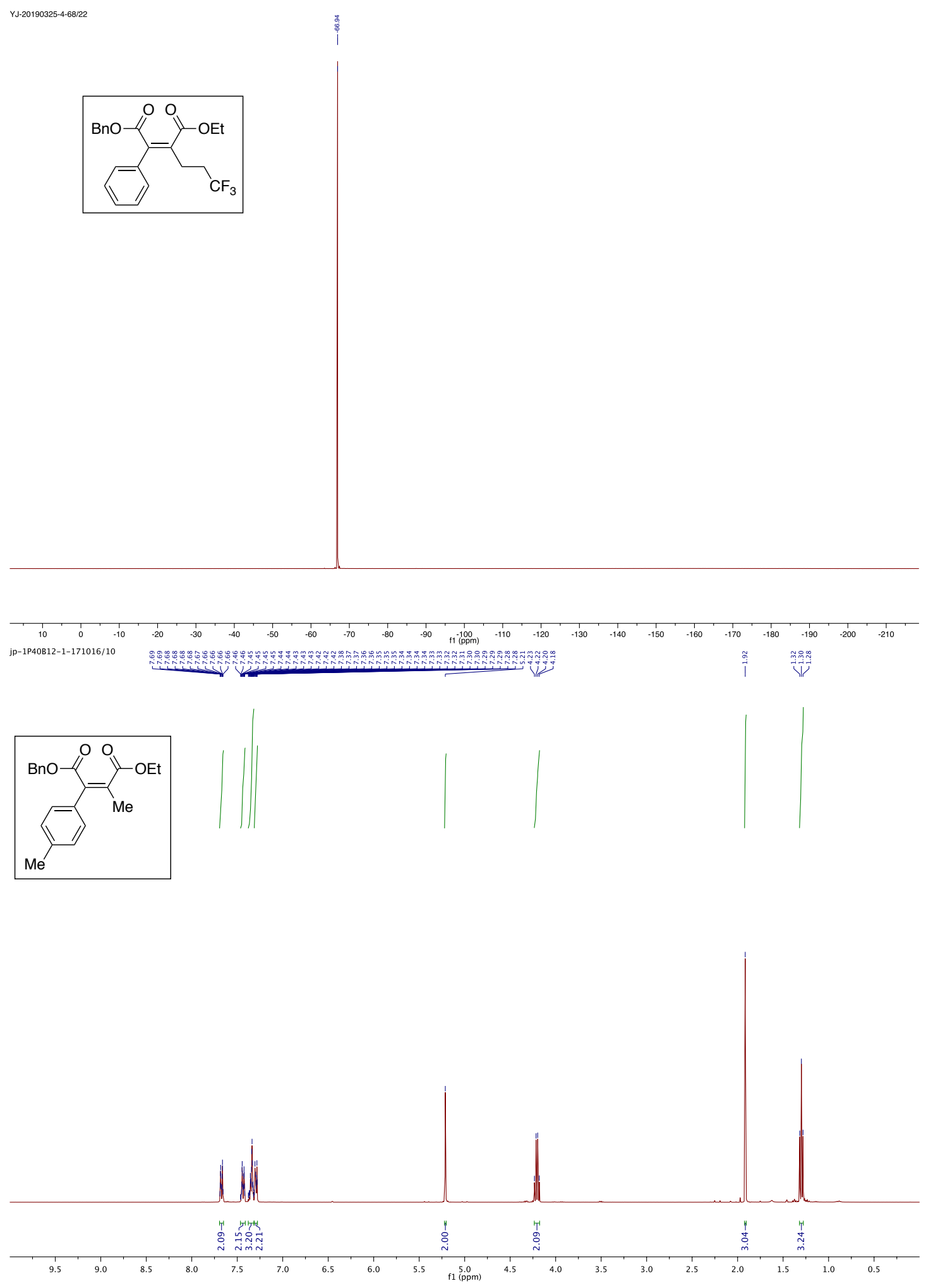

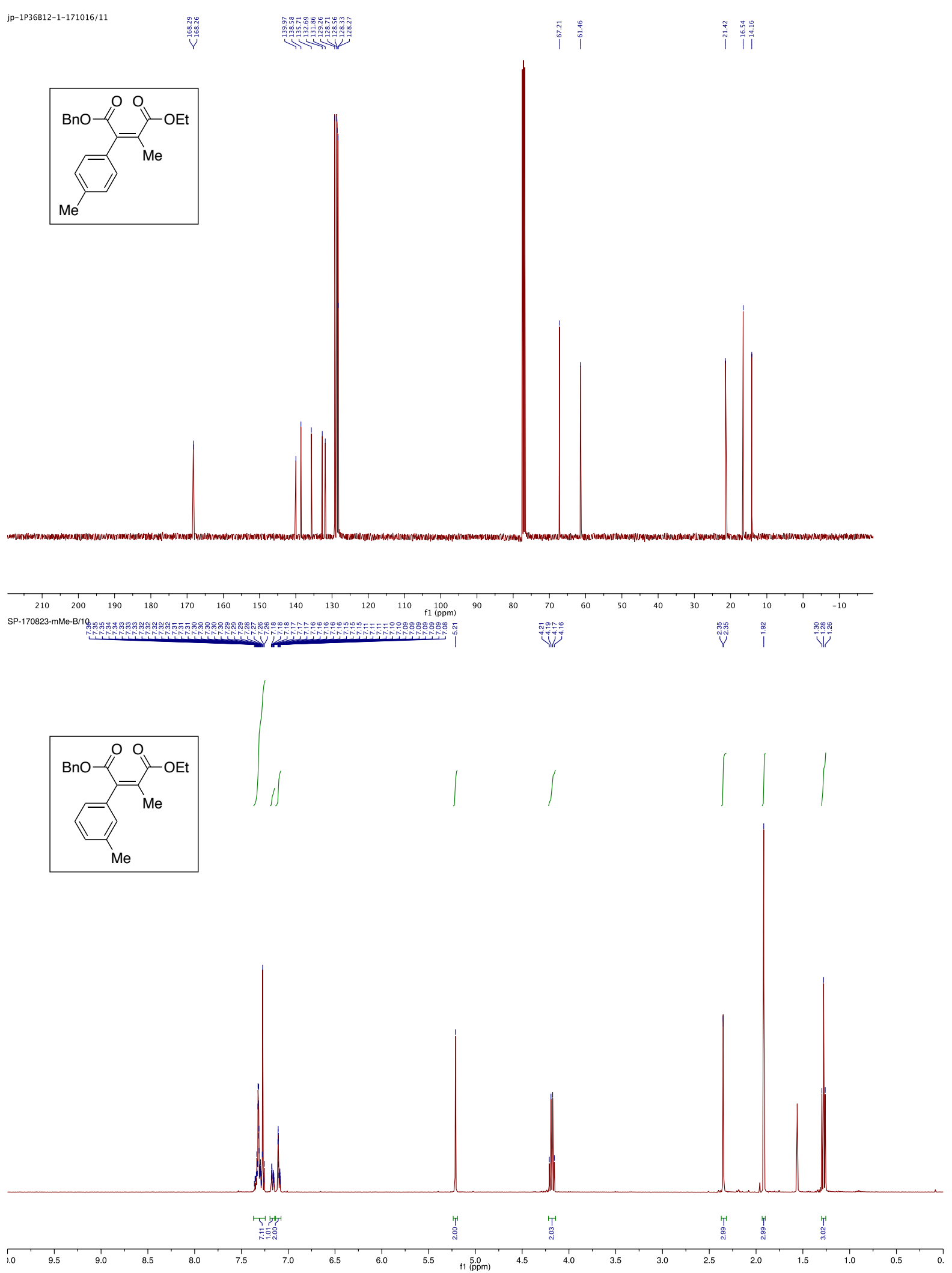

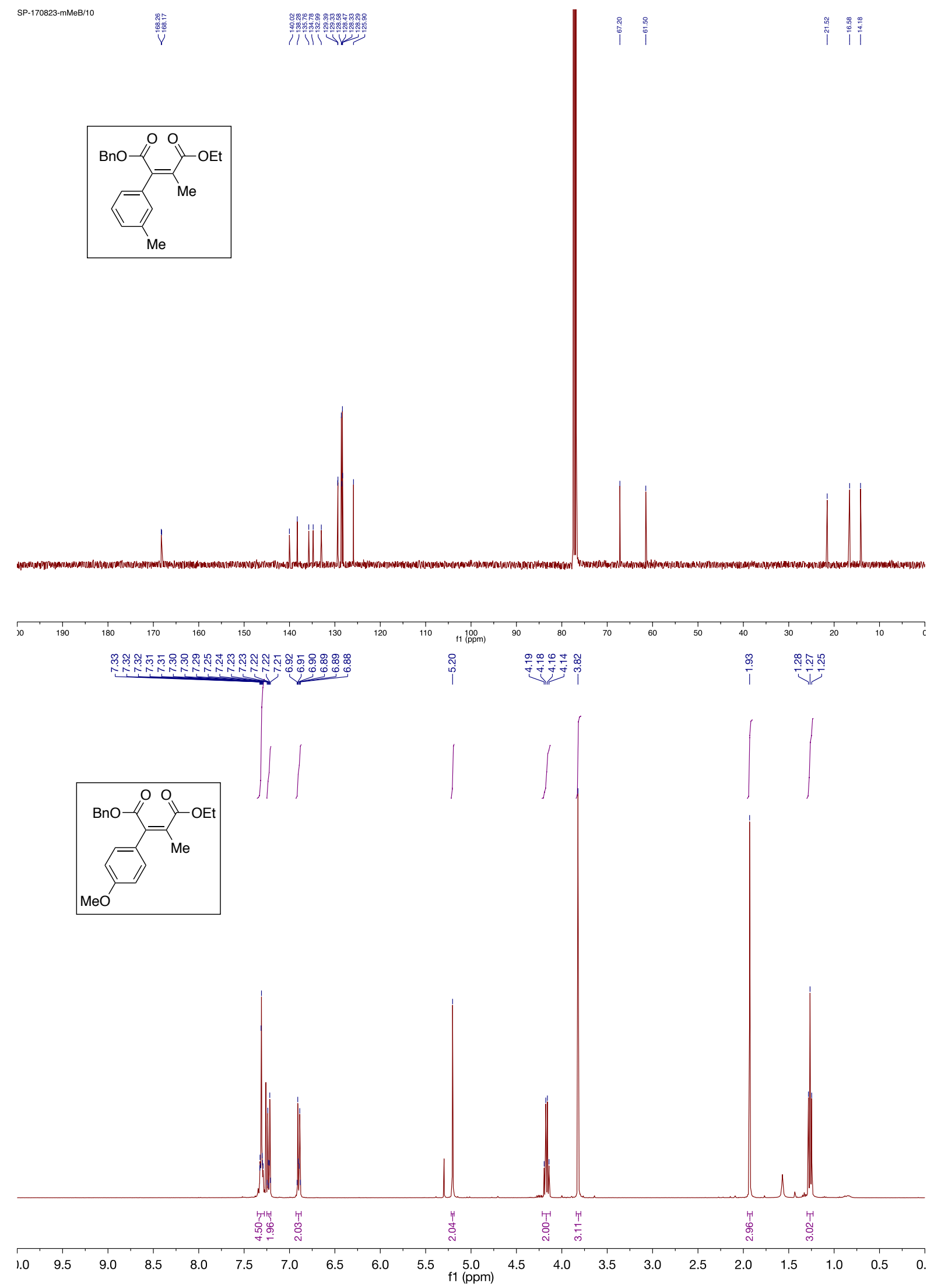


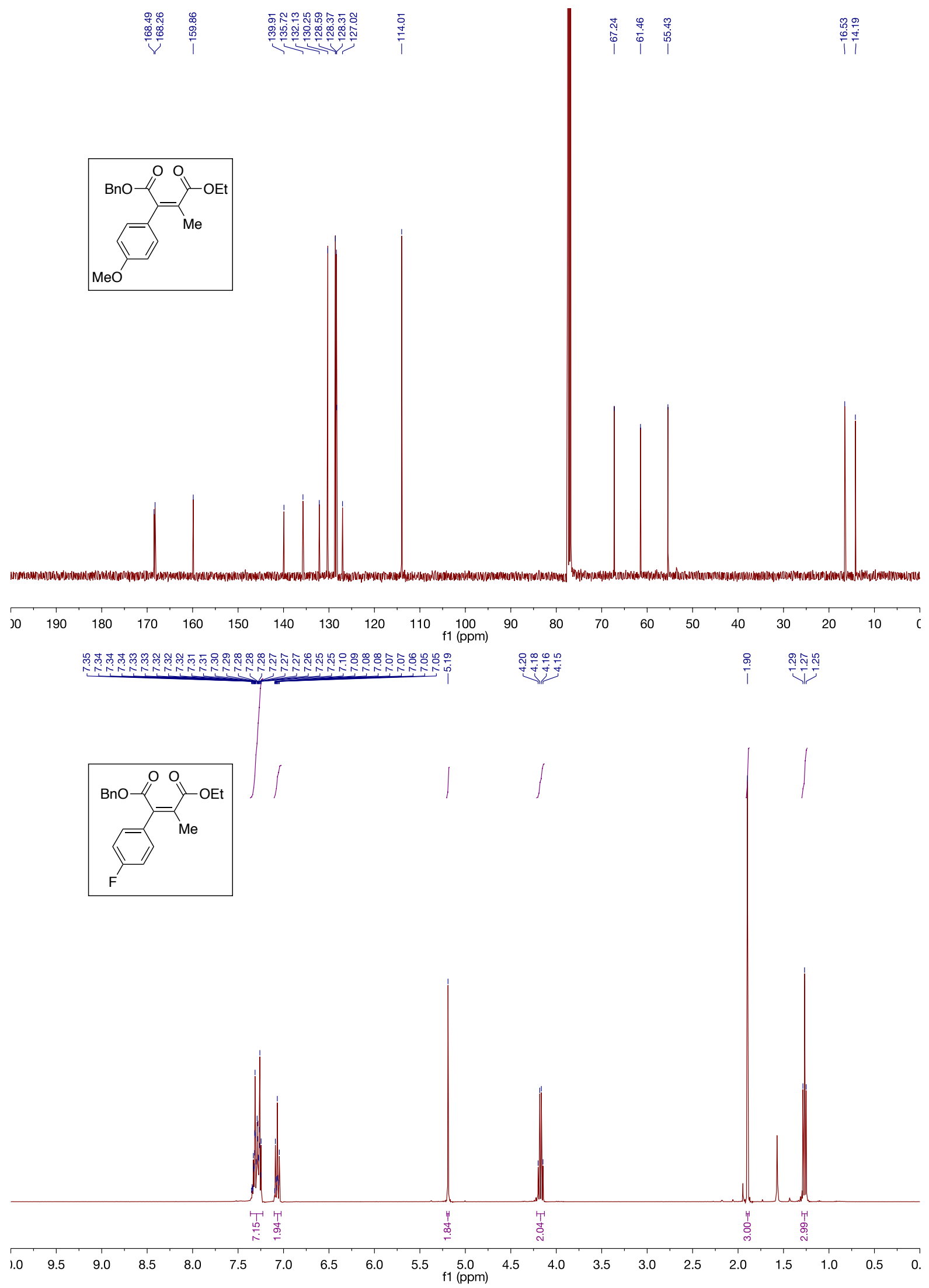




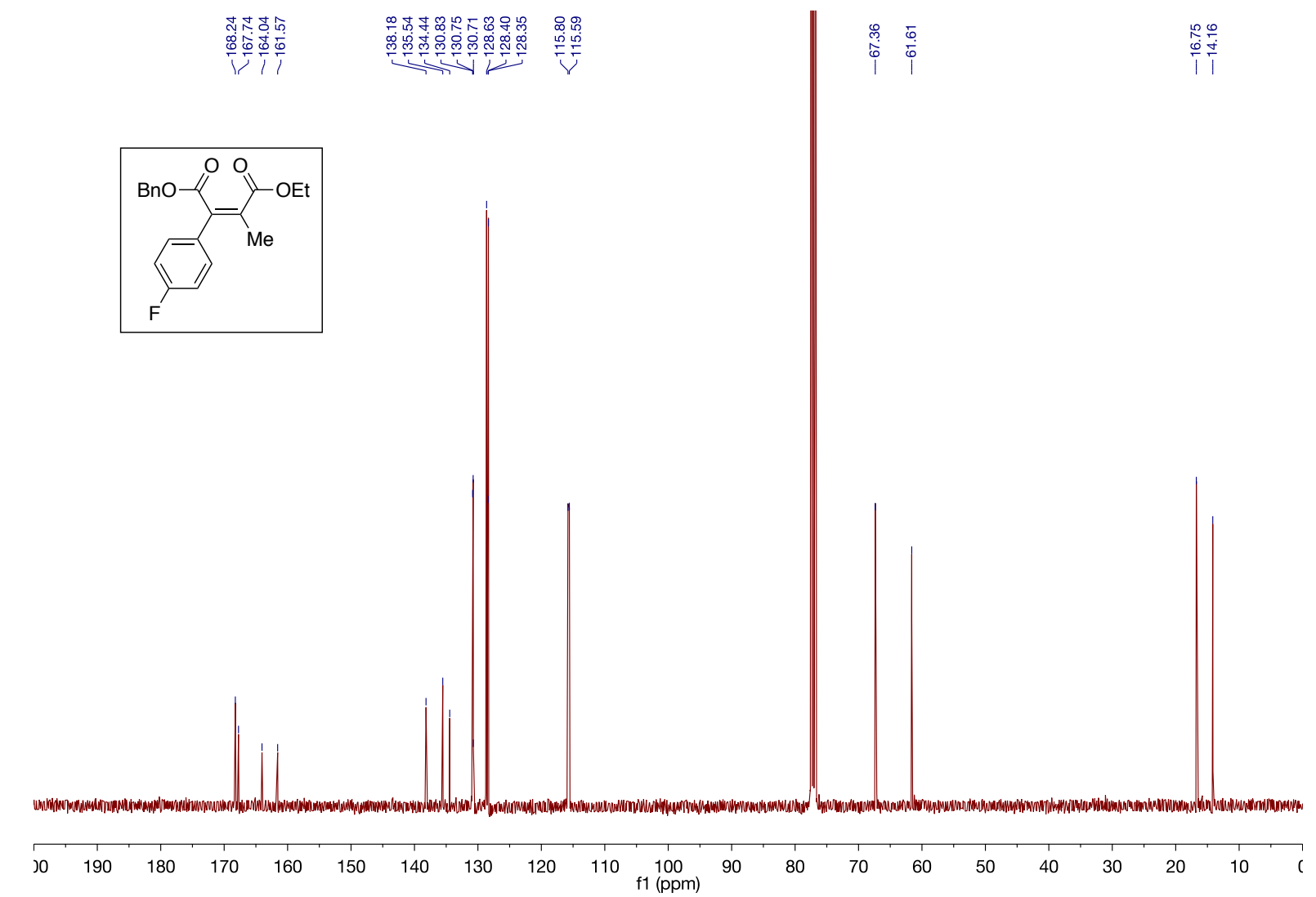

SP-180916-1n-F19/10

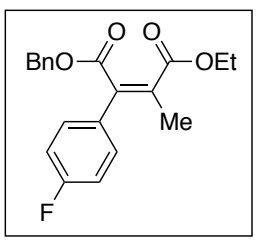



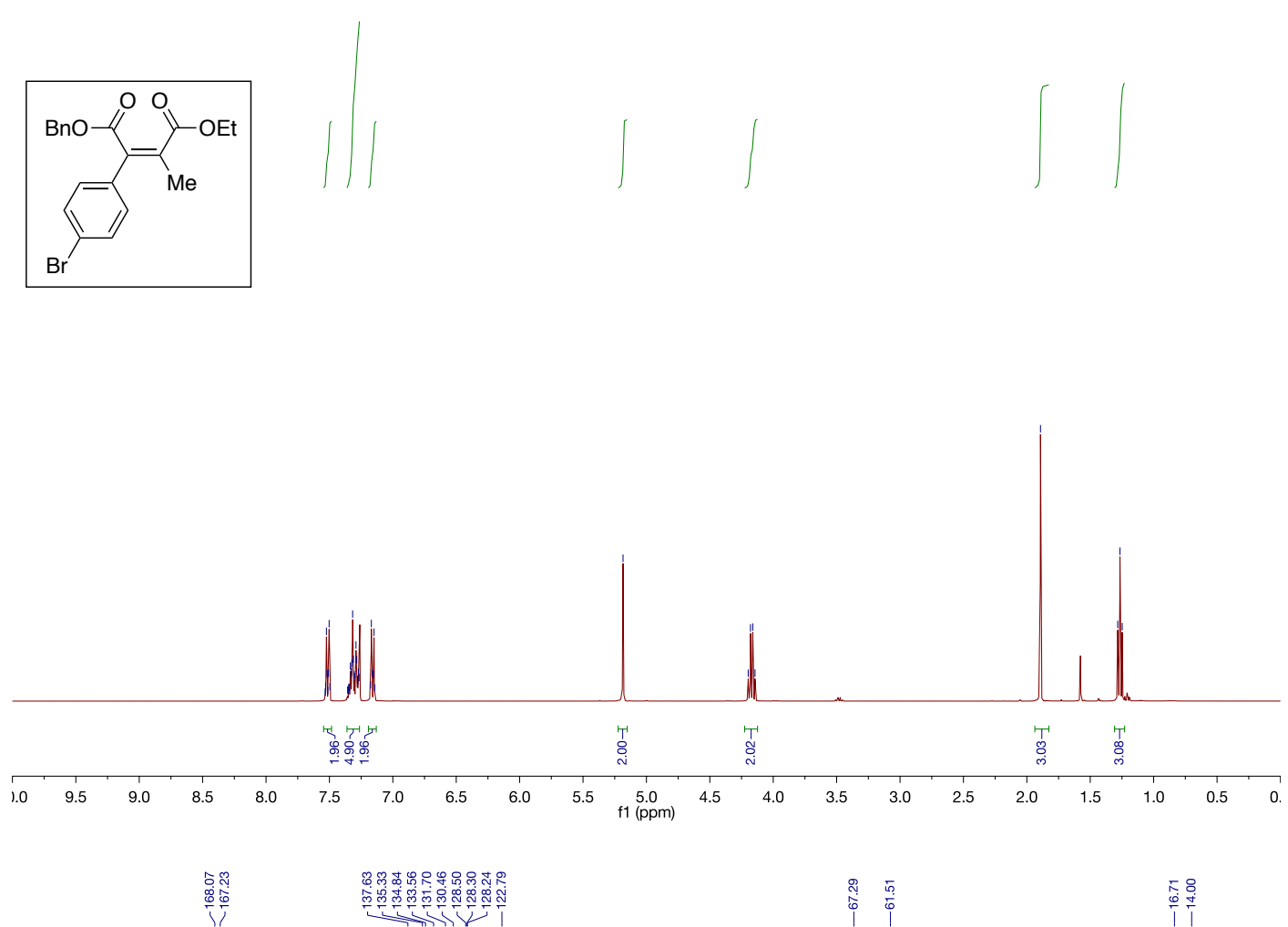

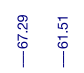
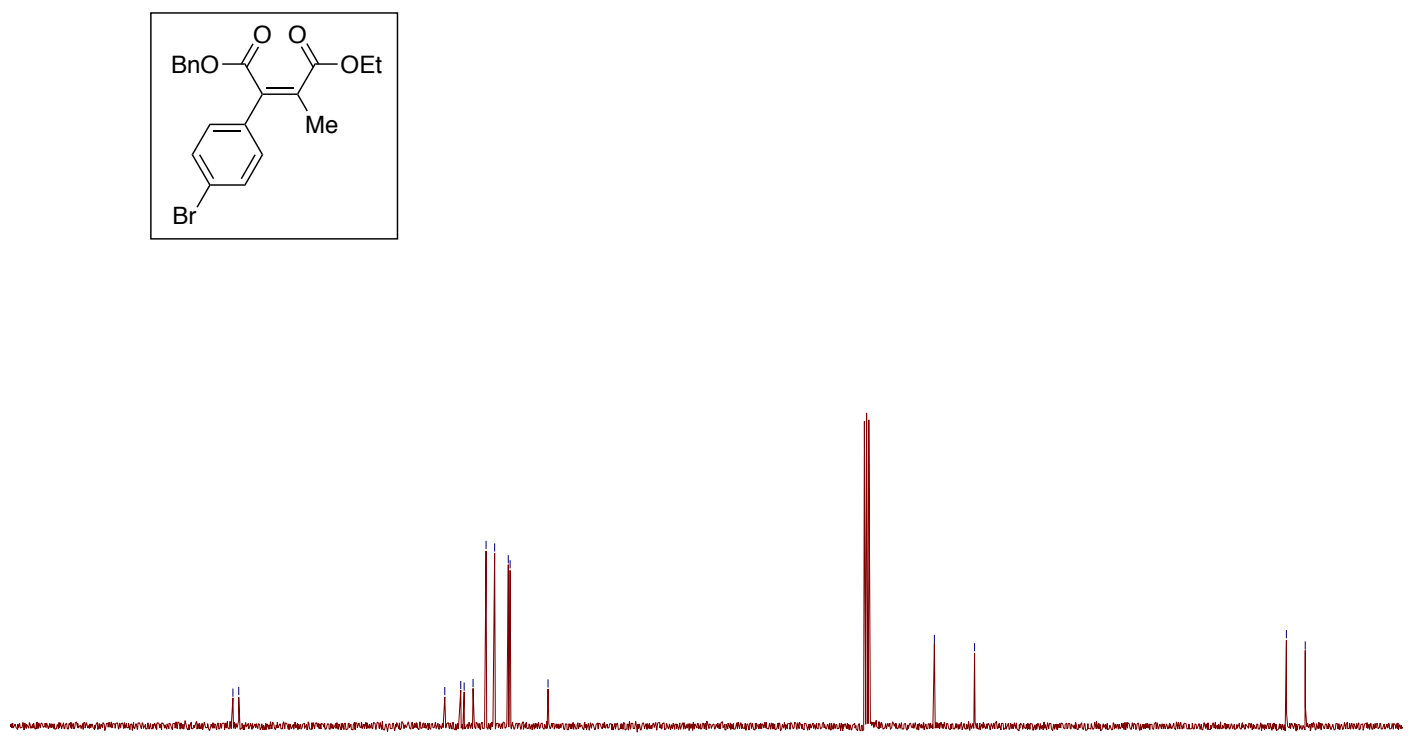

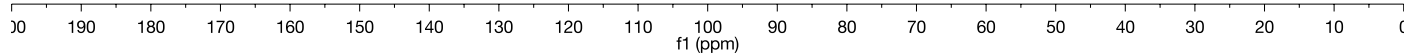



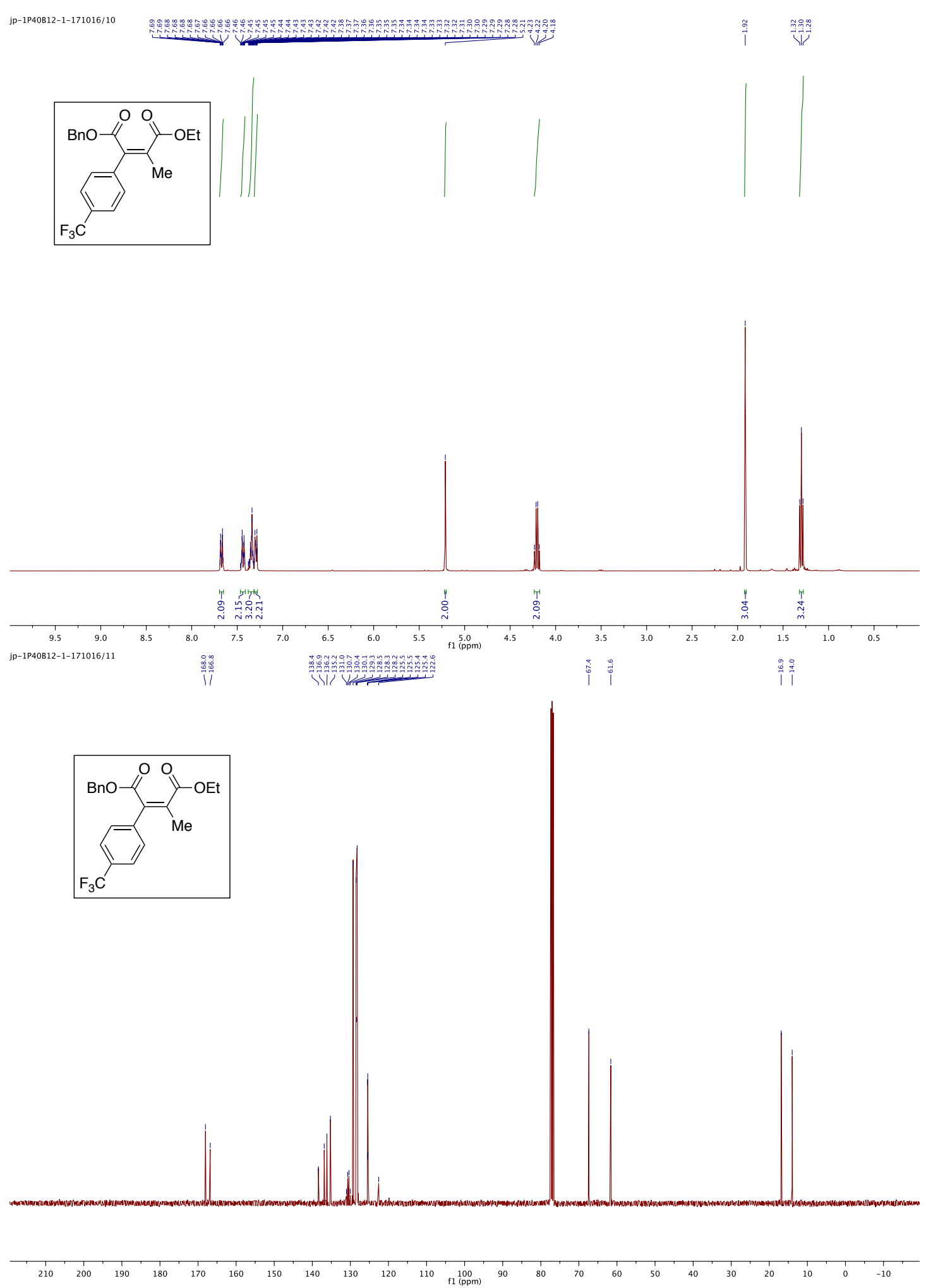


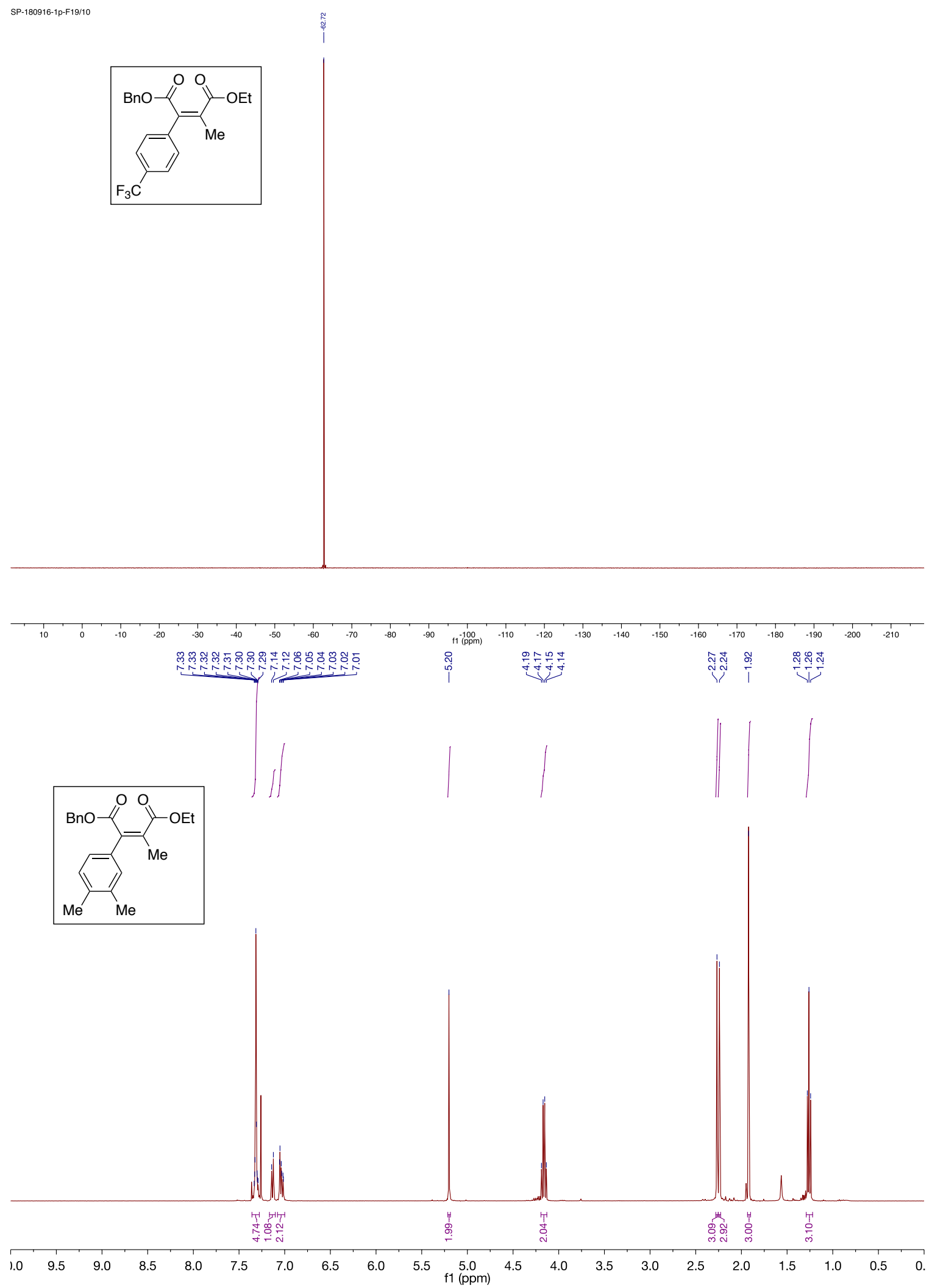




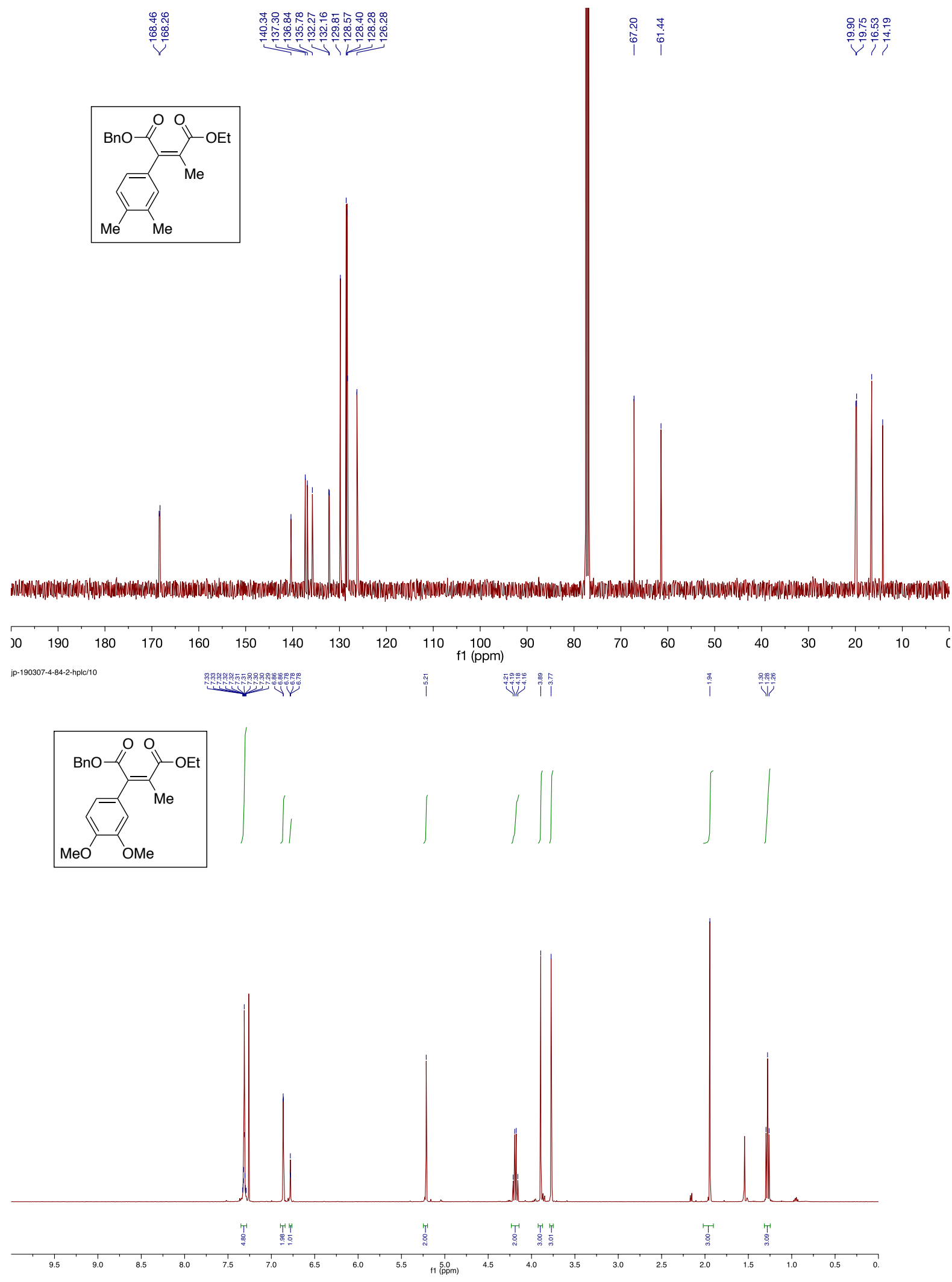



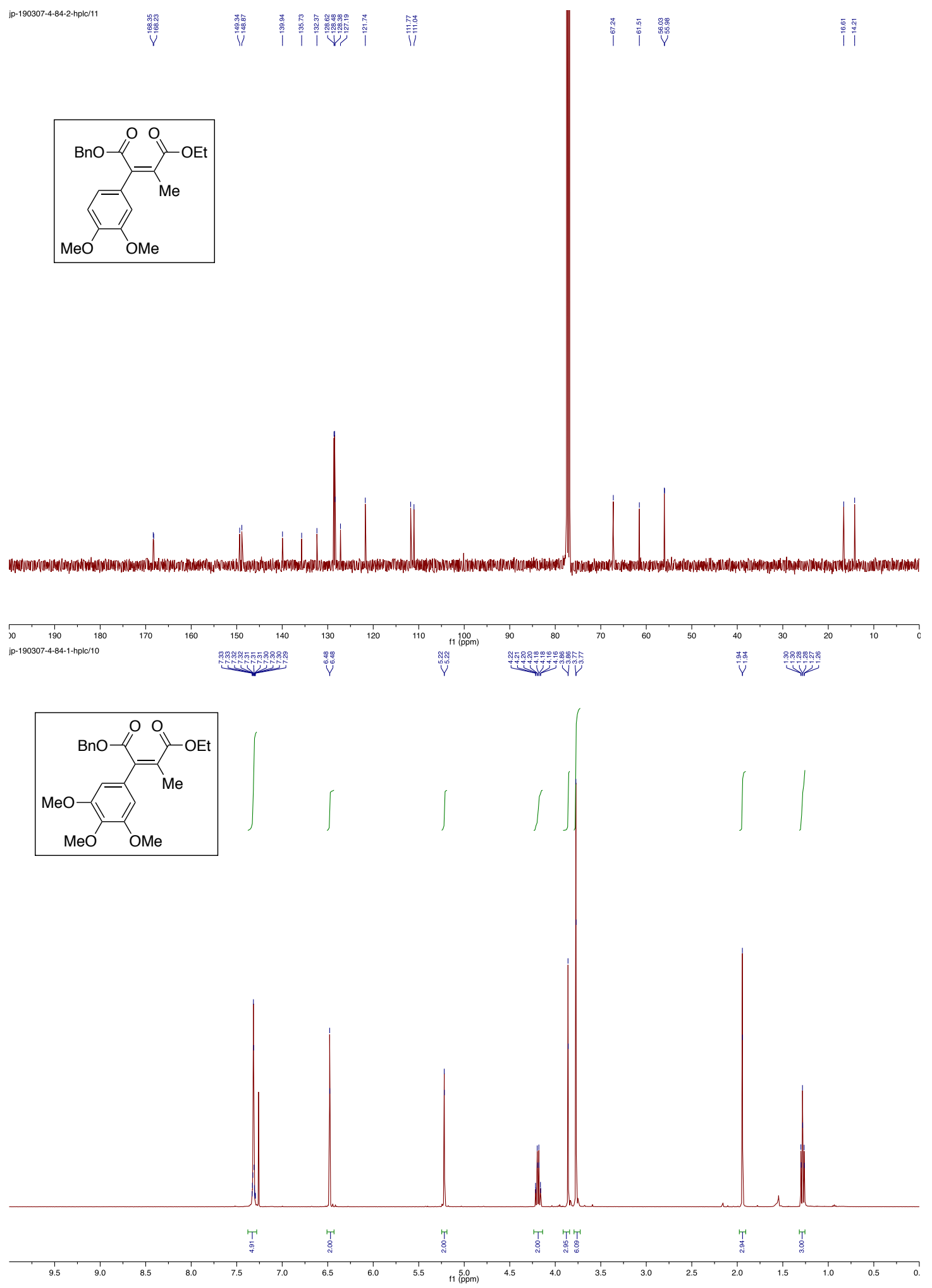

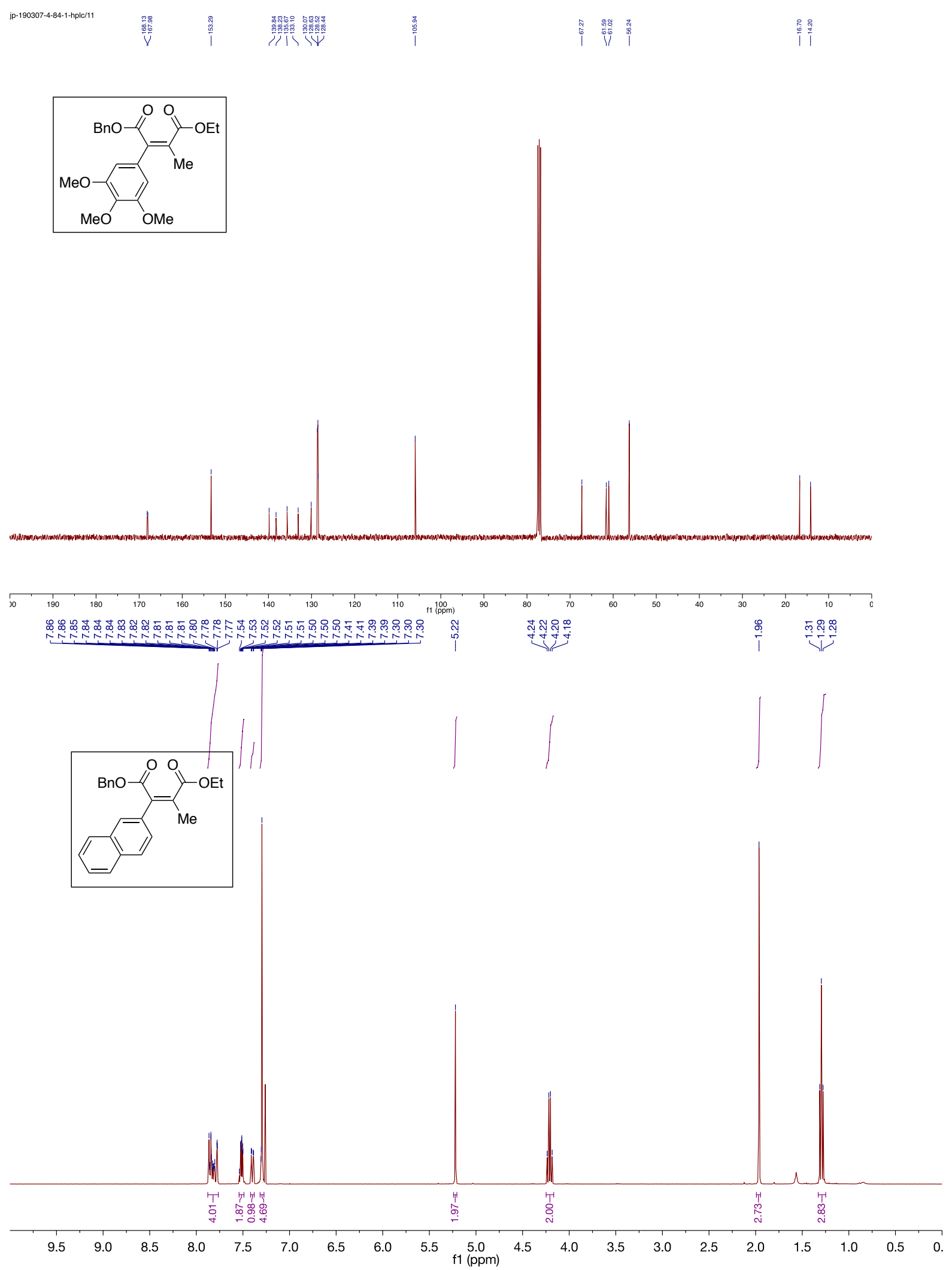
$\overline{5}$.
$0 \%$
00
$\vdots 0$

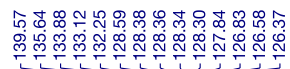
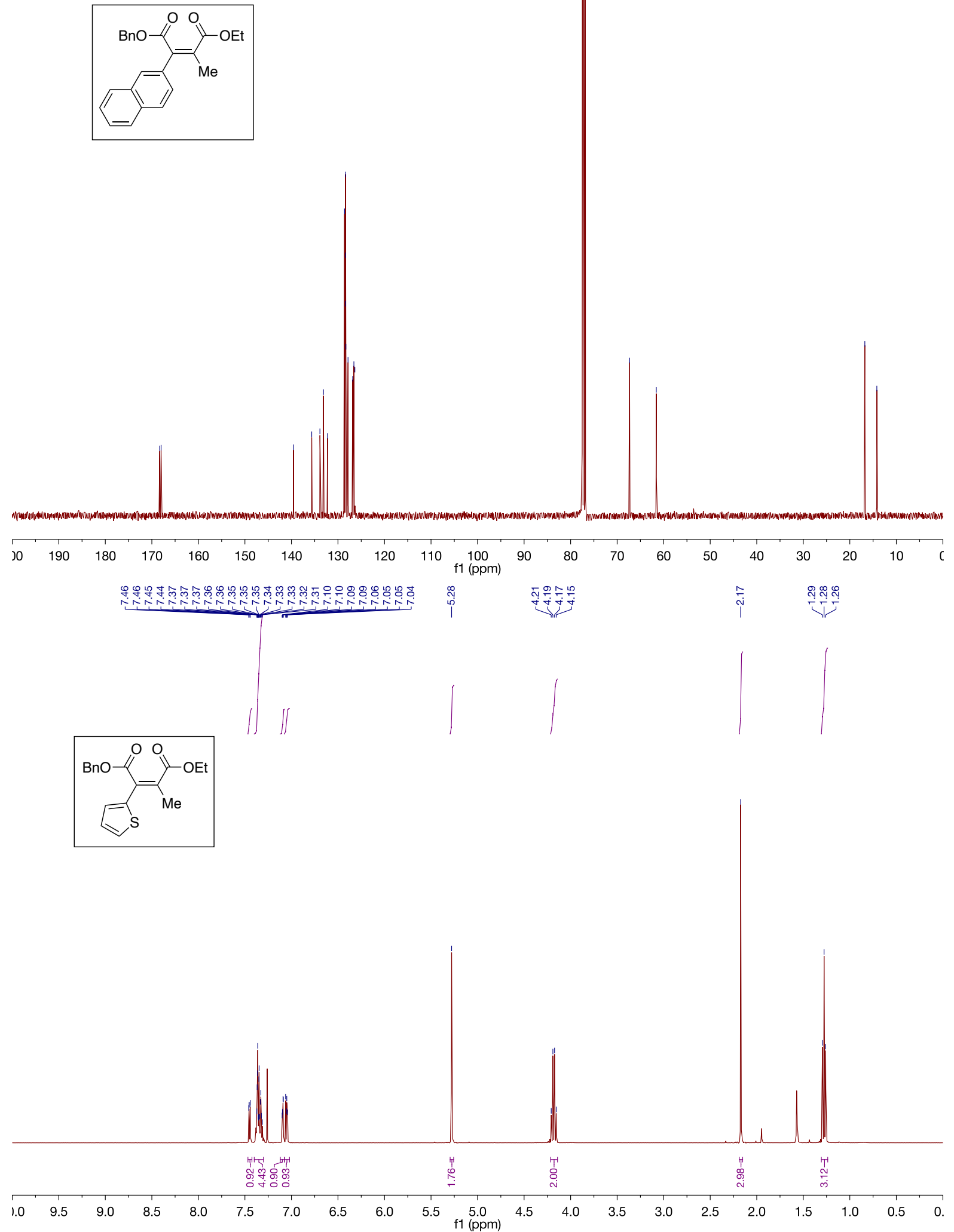

S-93 


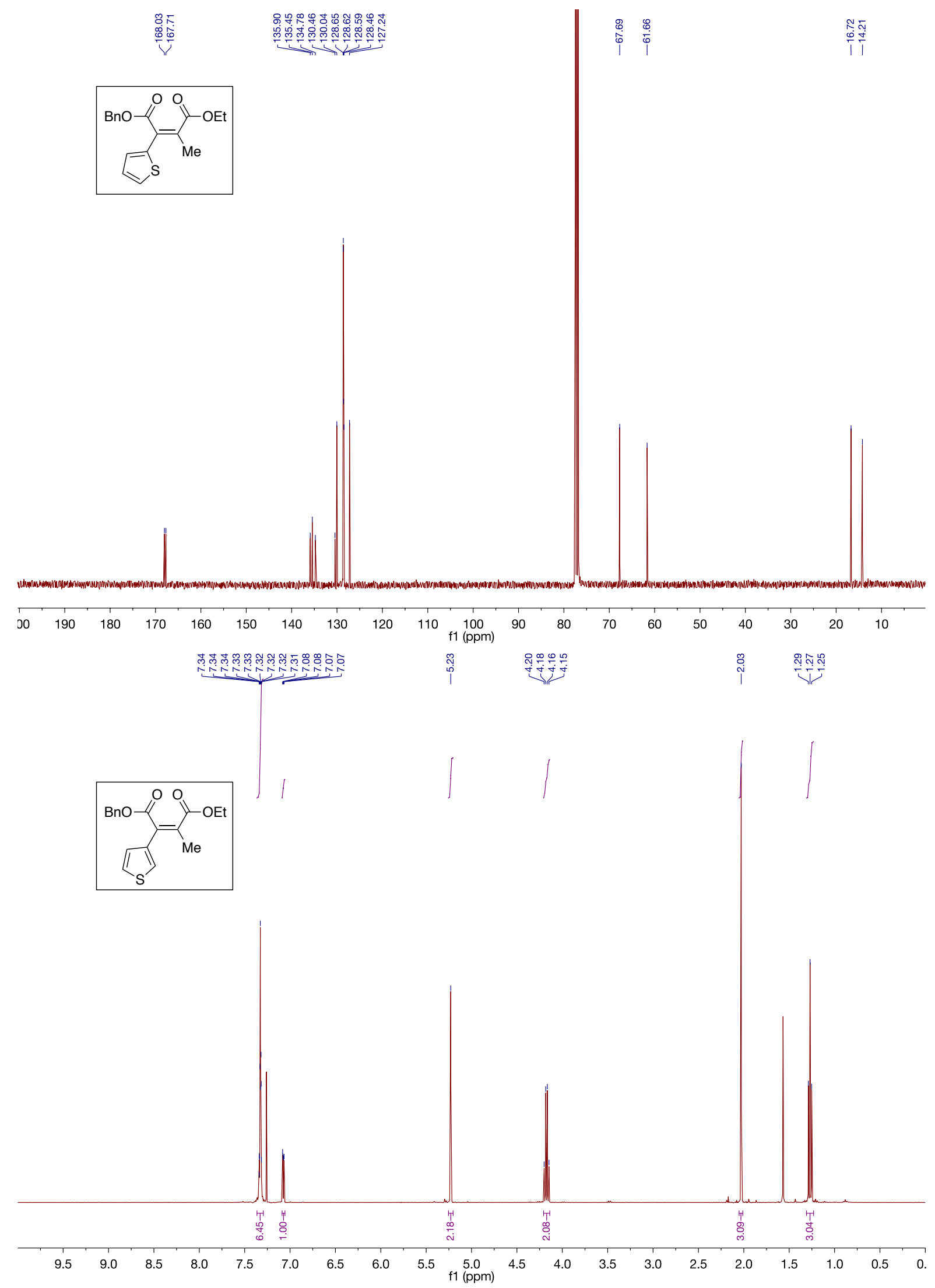




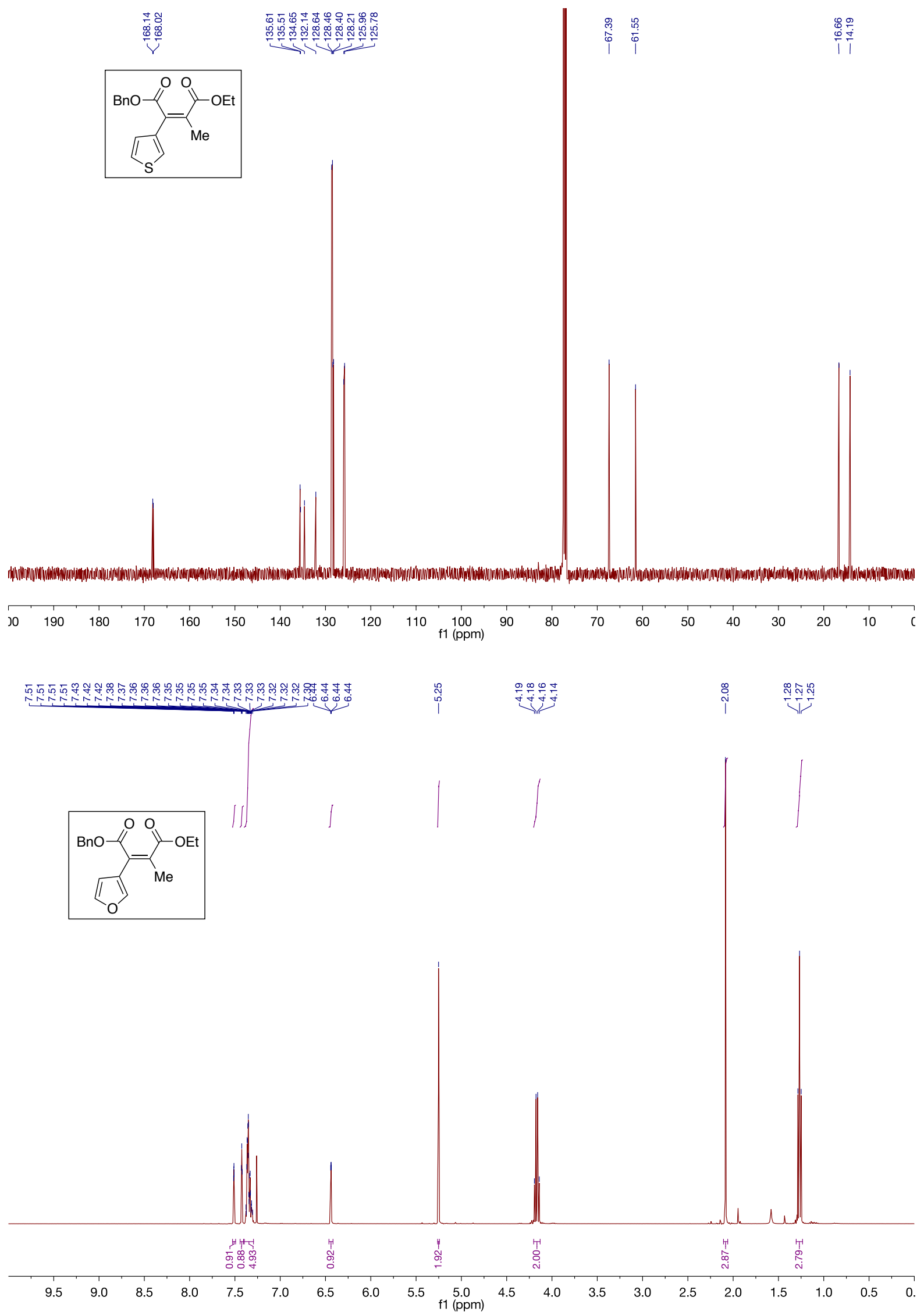




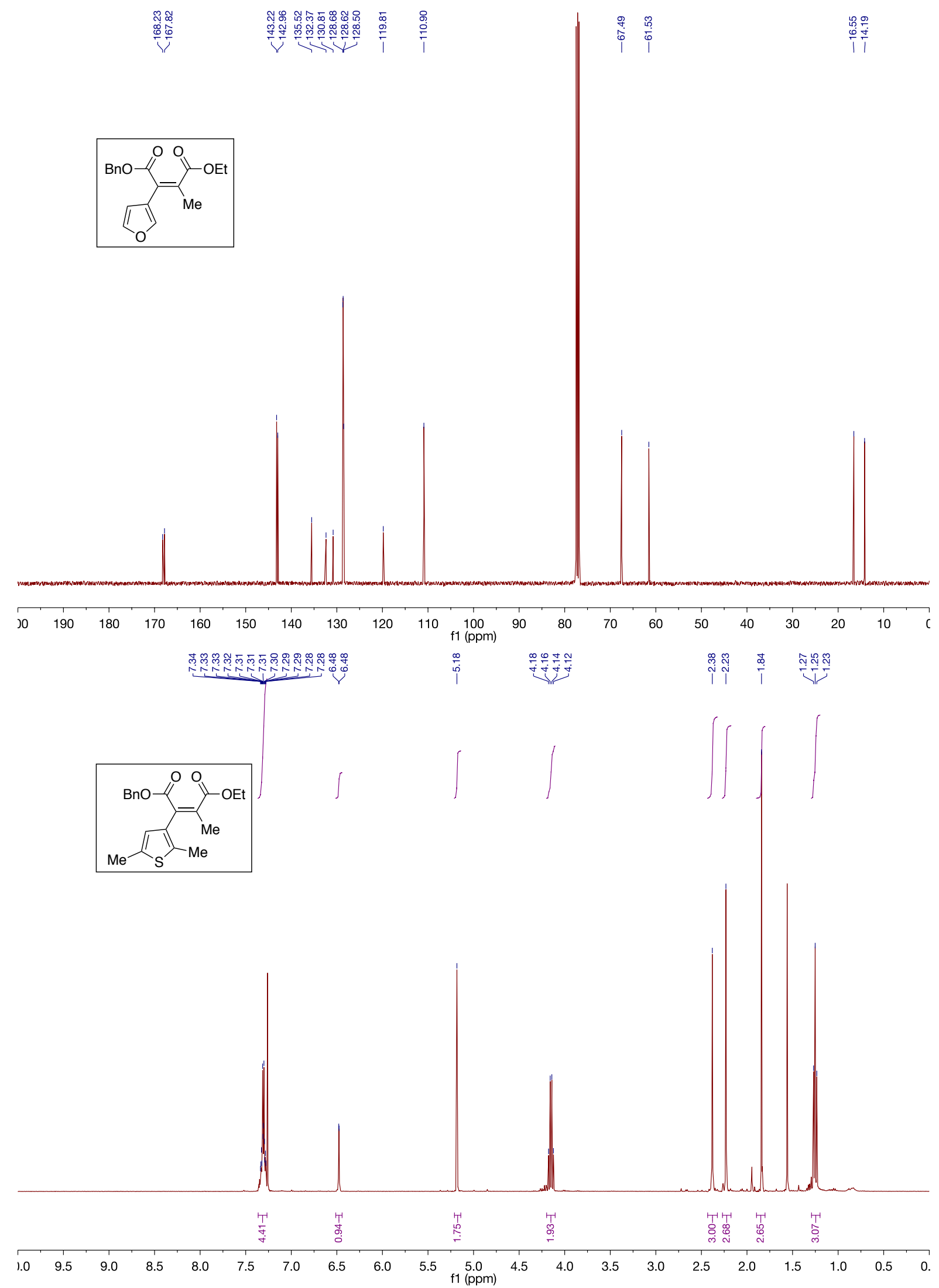



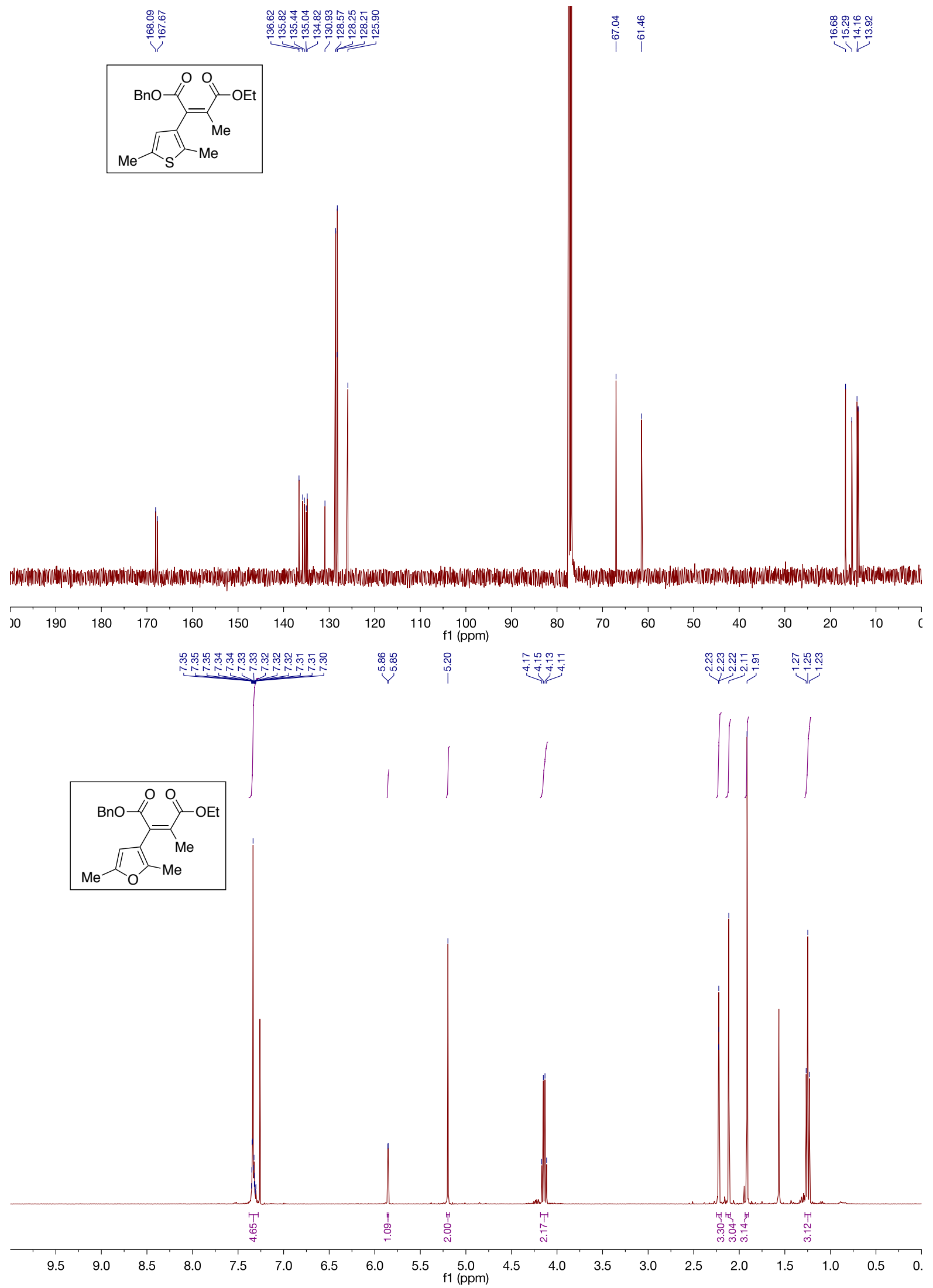


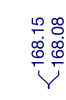

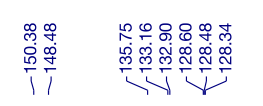

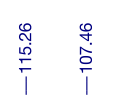

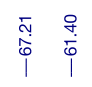

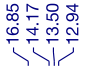

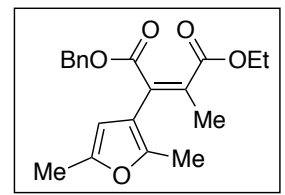
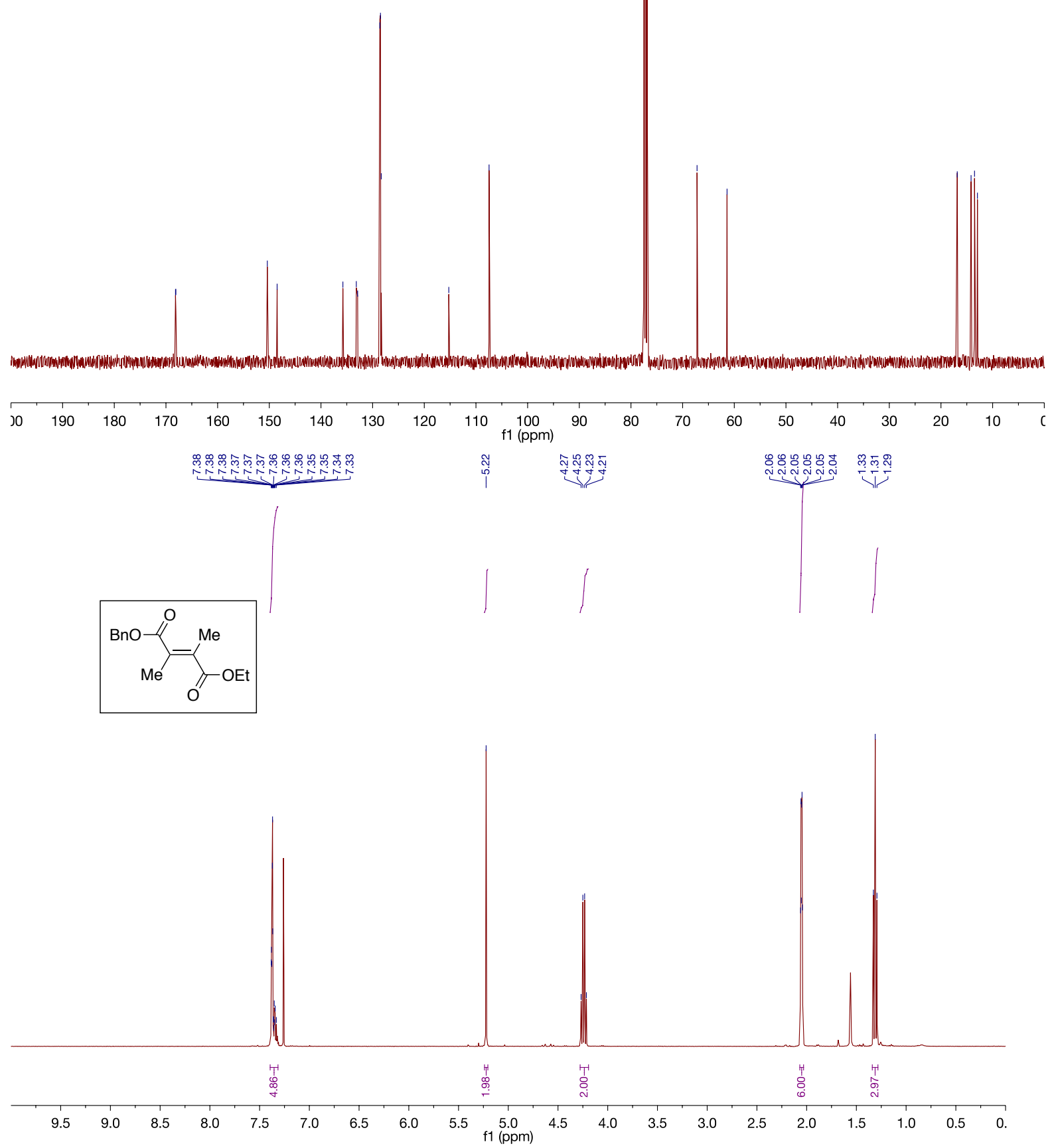


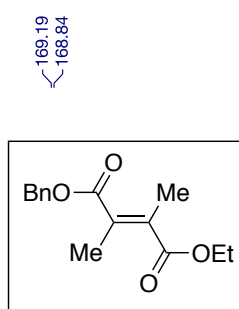

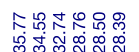
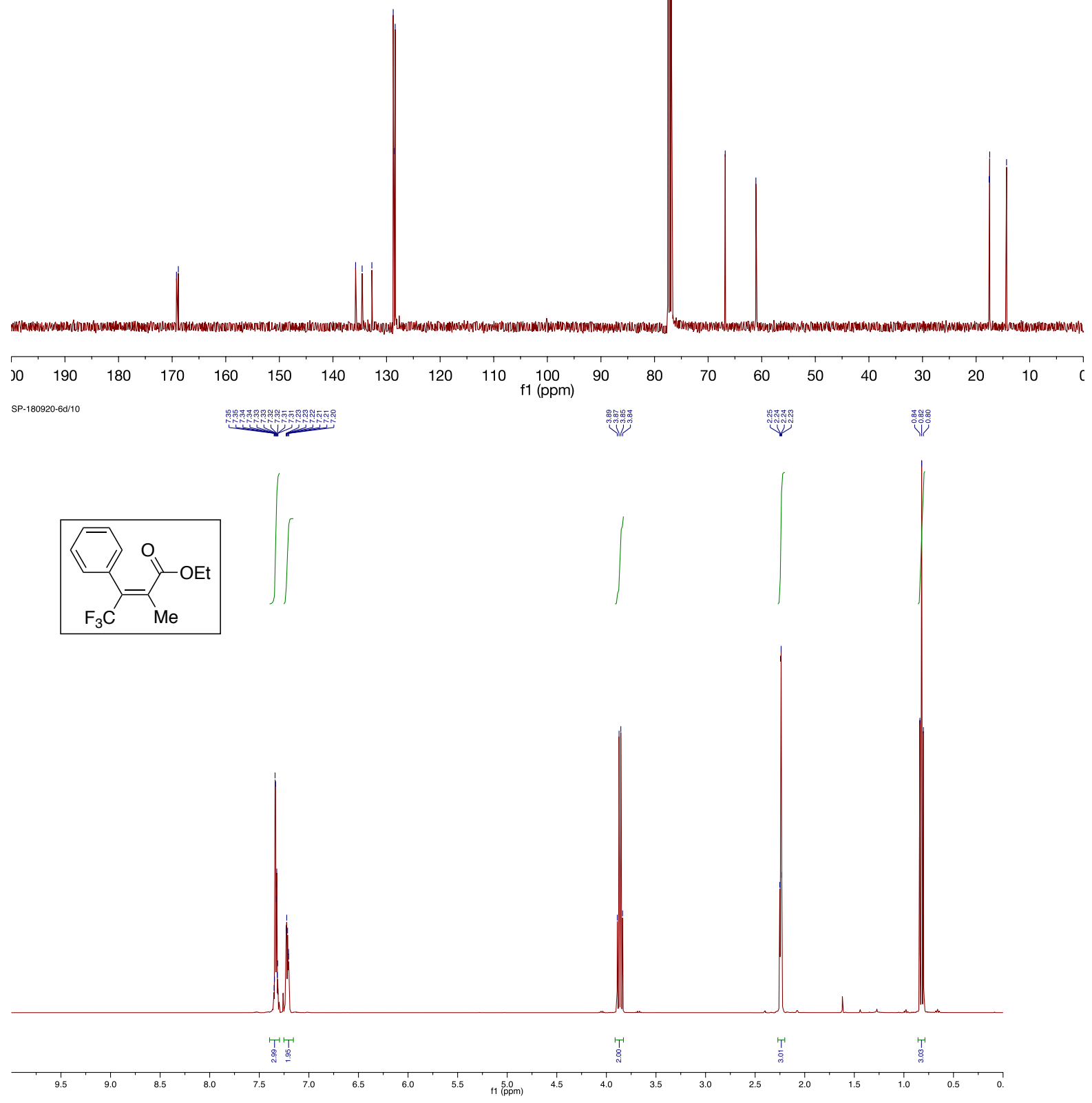

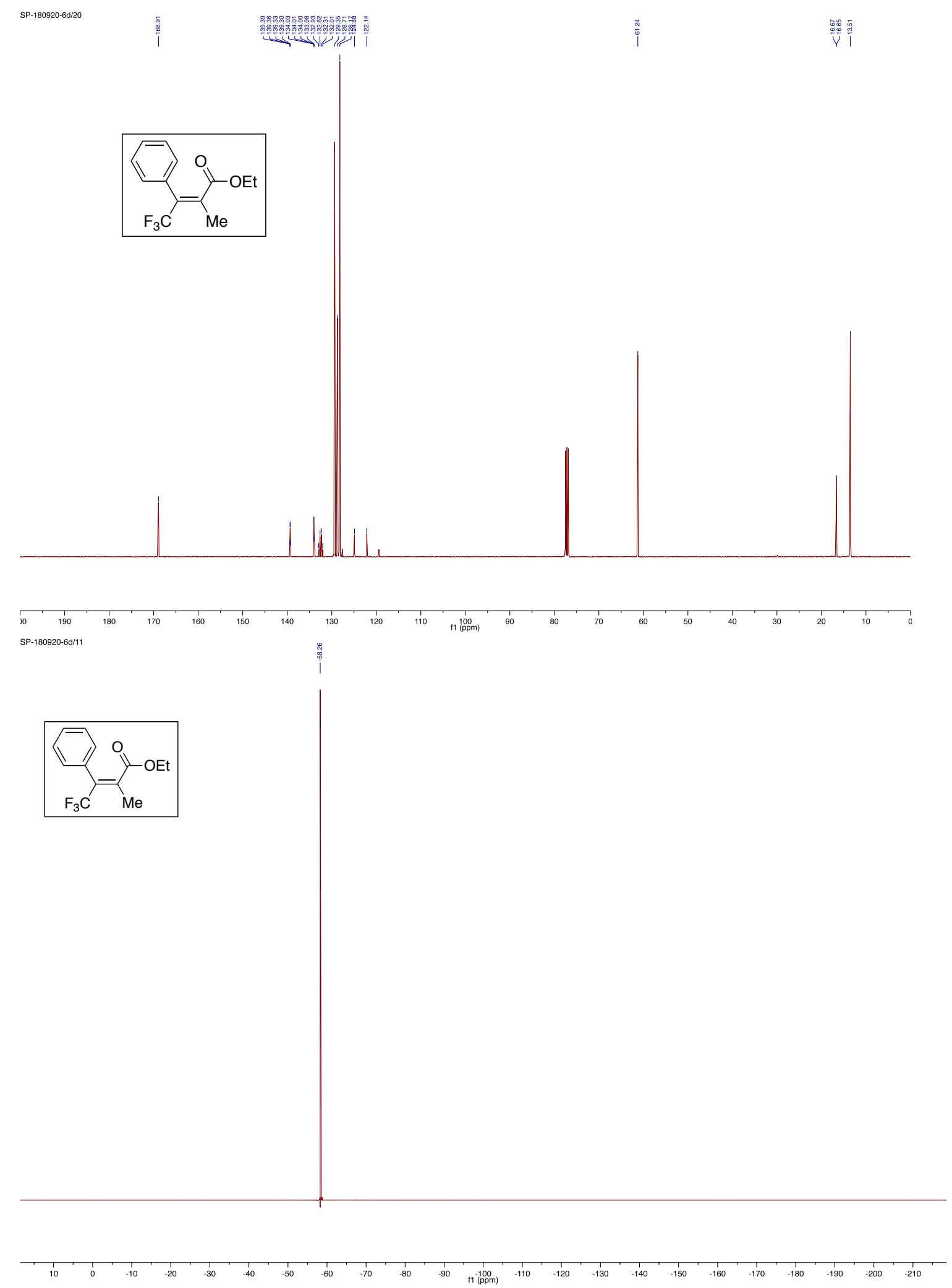
ip-180126-kim-CF3/10

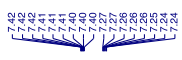

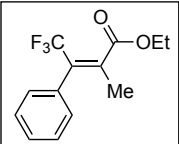

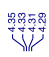

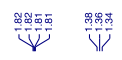

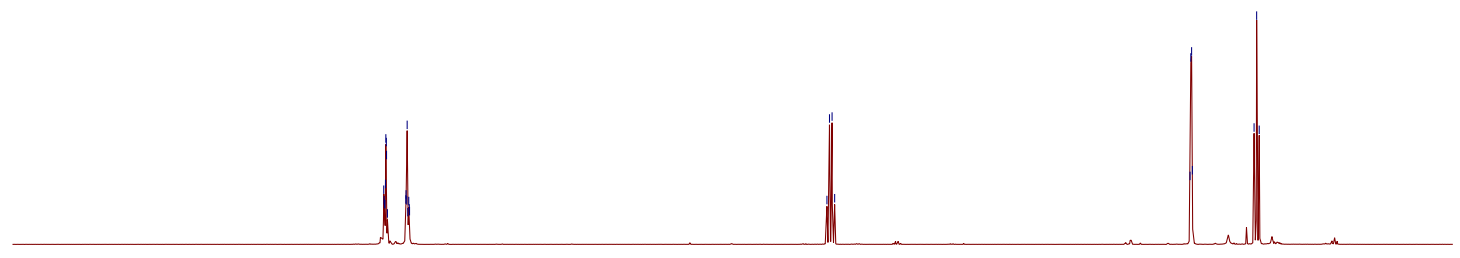

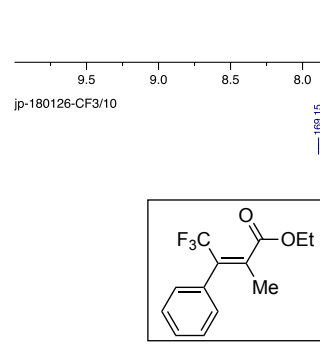

变

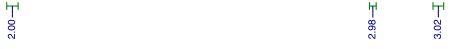

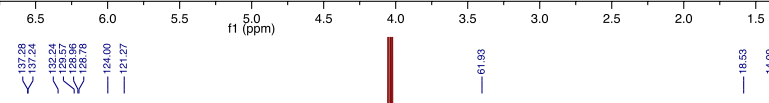

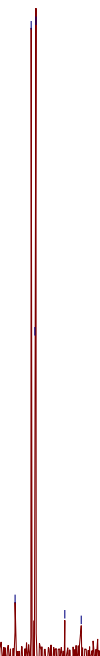

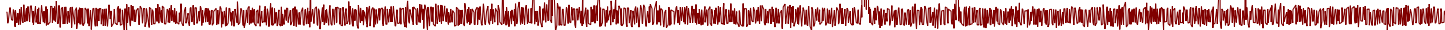

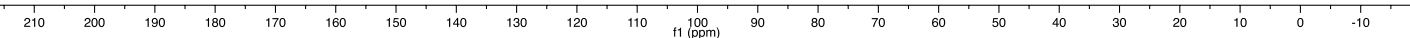

S-101 

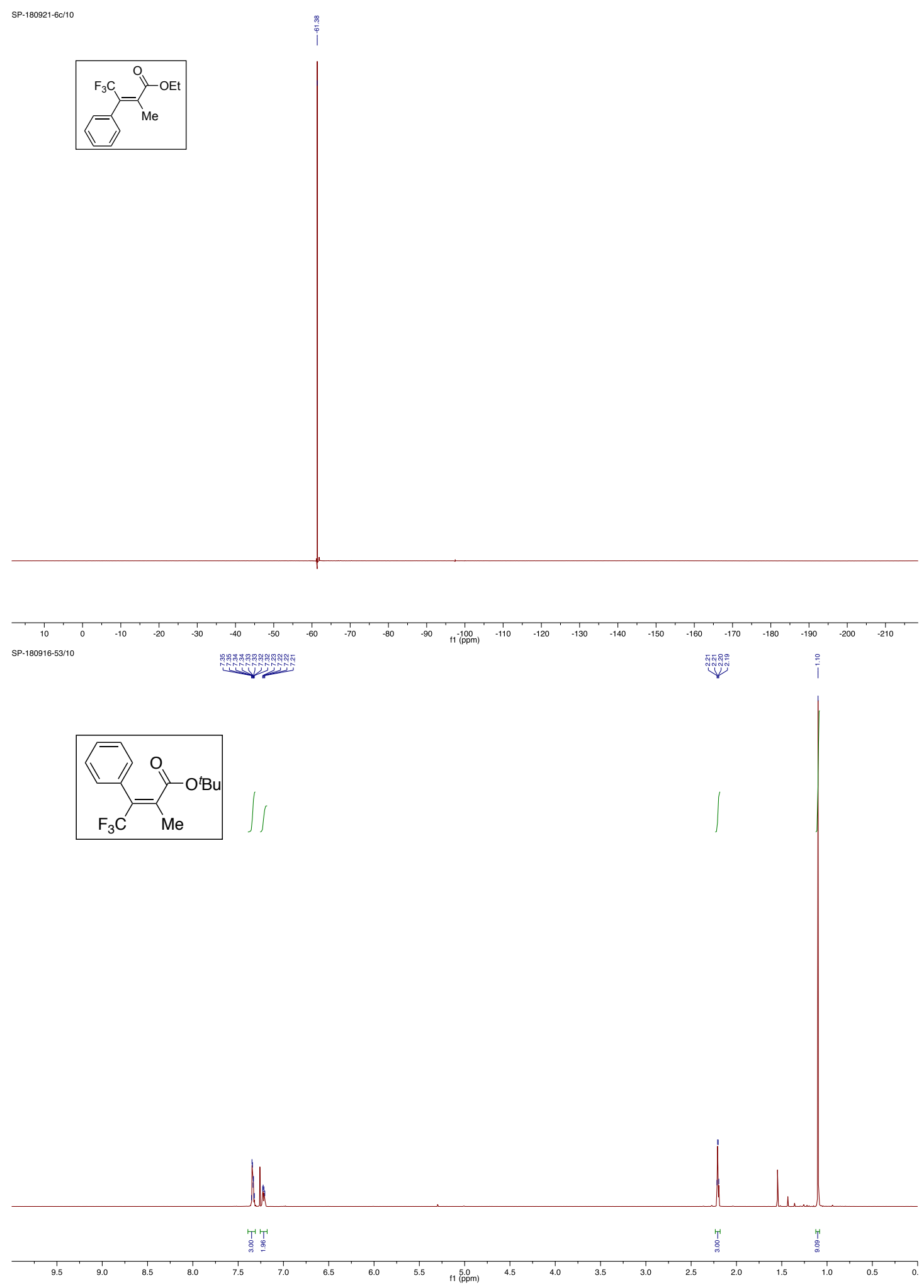

S-102 

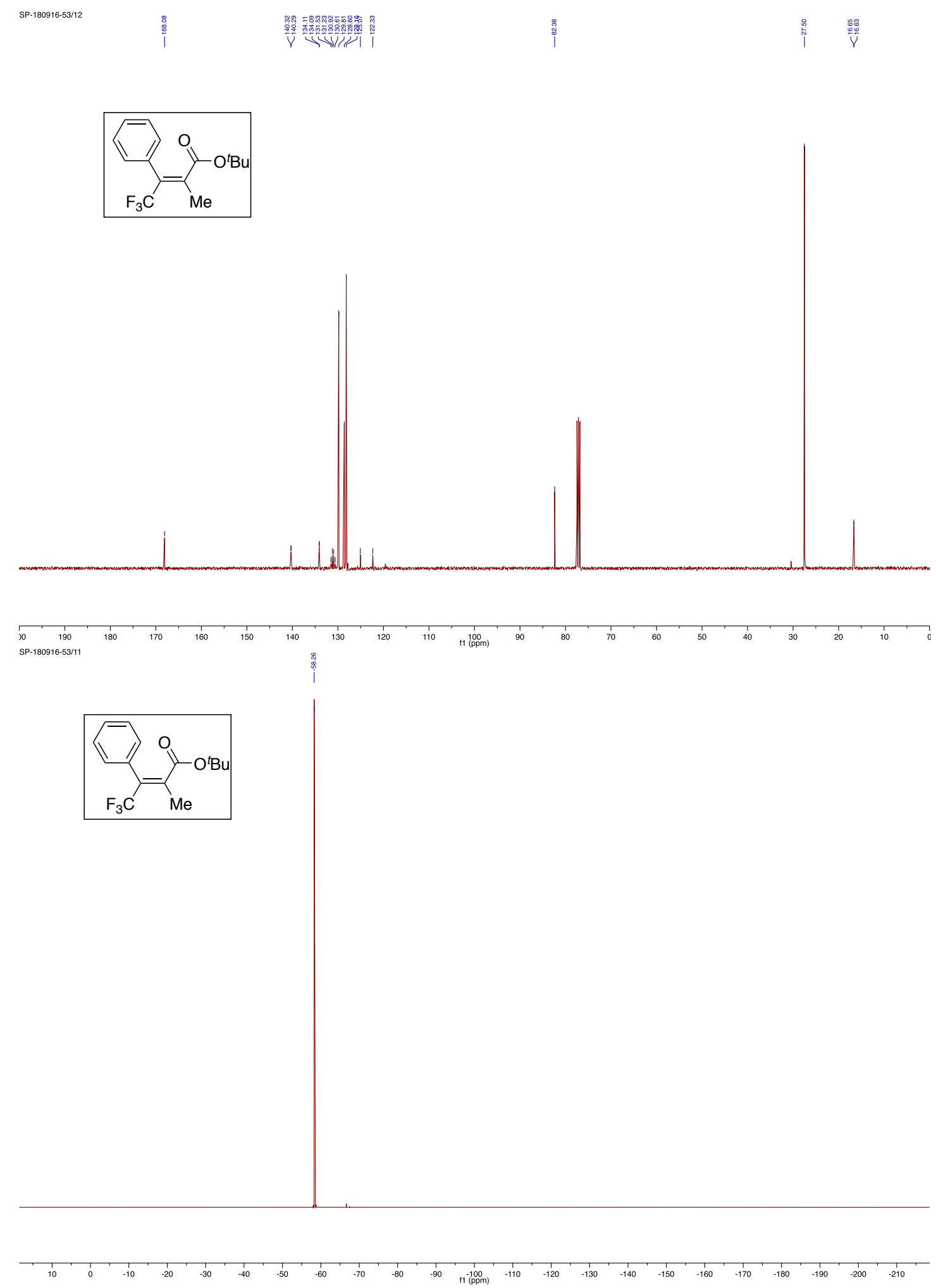

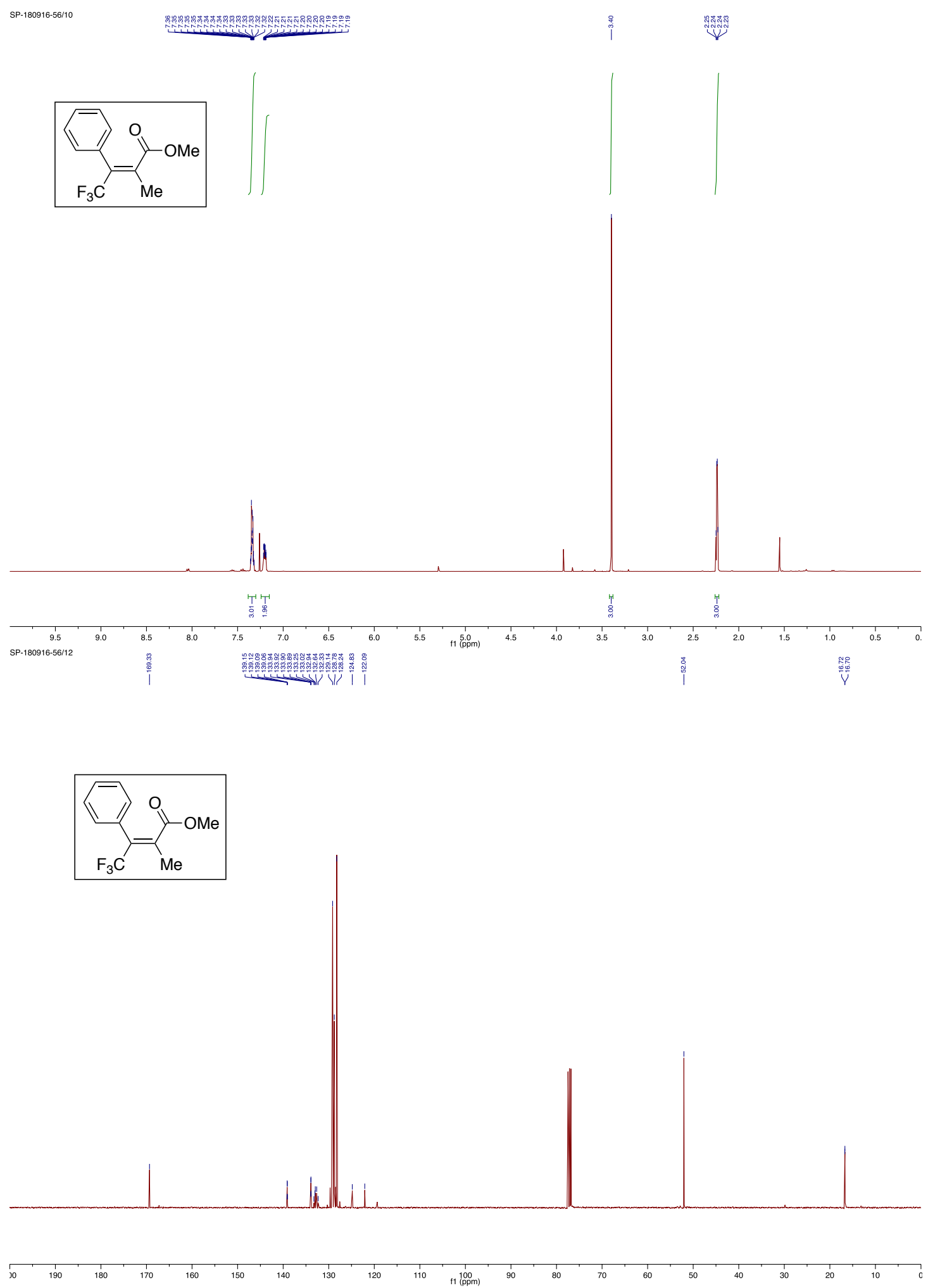

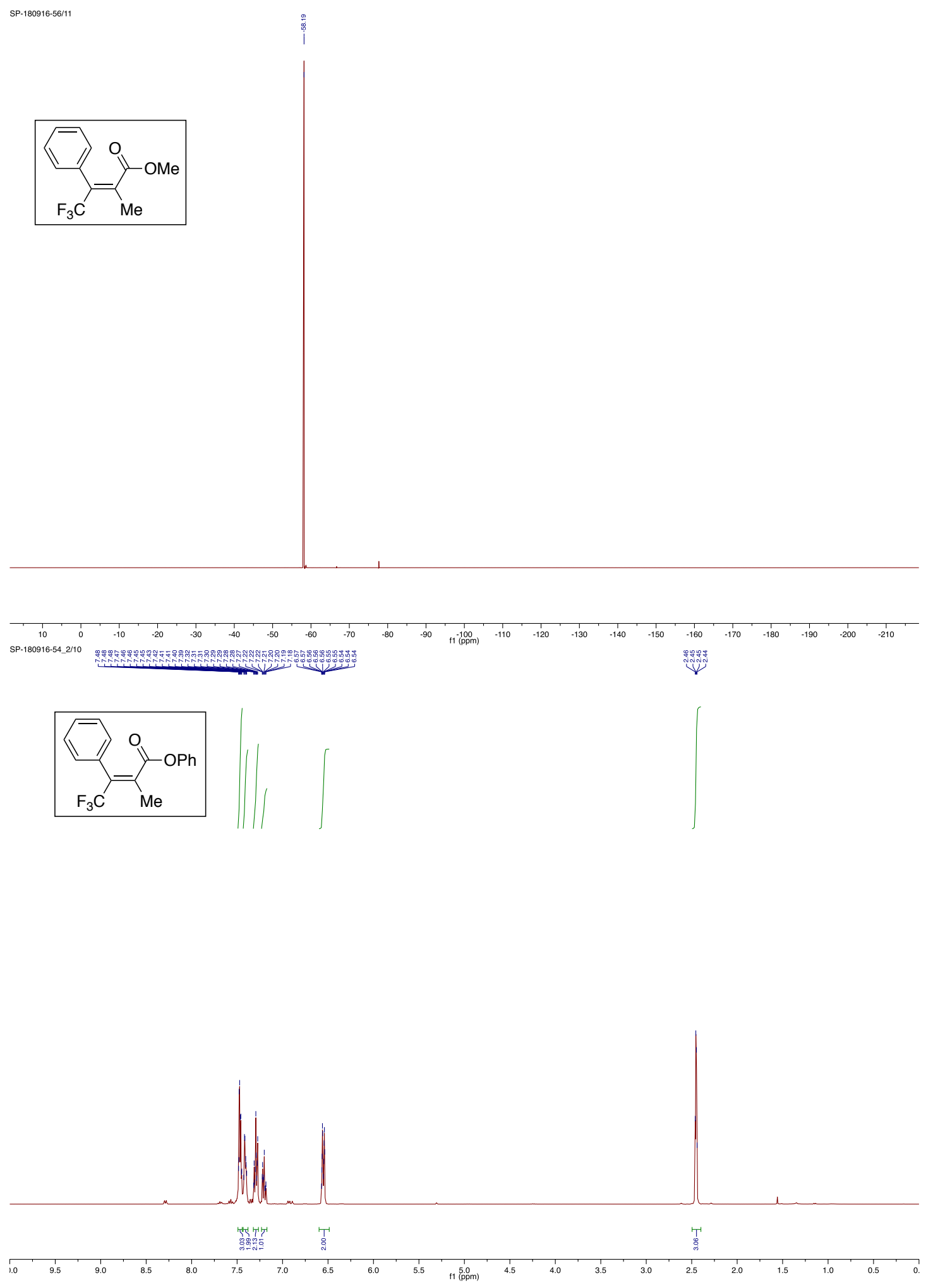
SP-180916-54/12
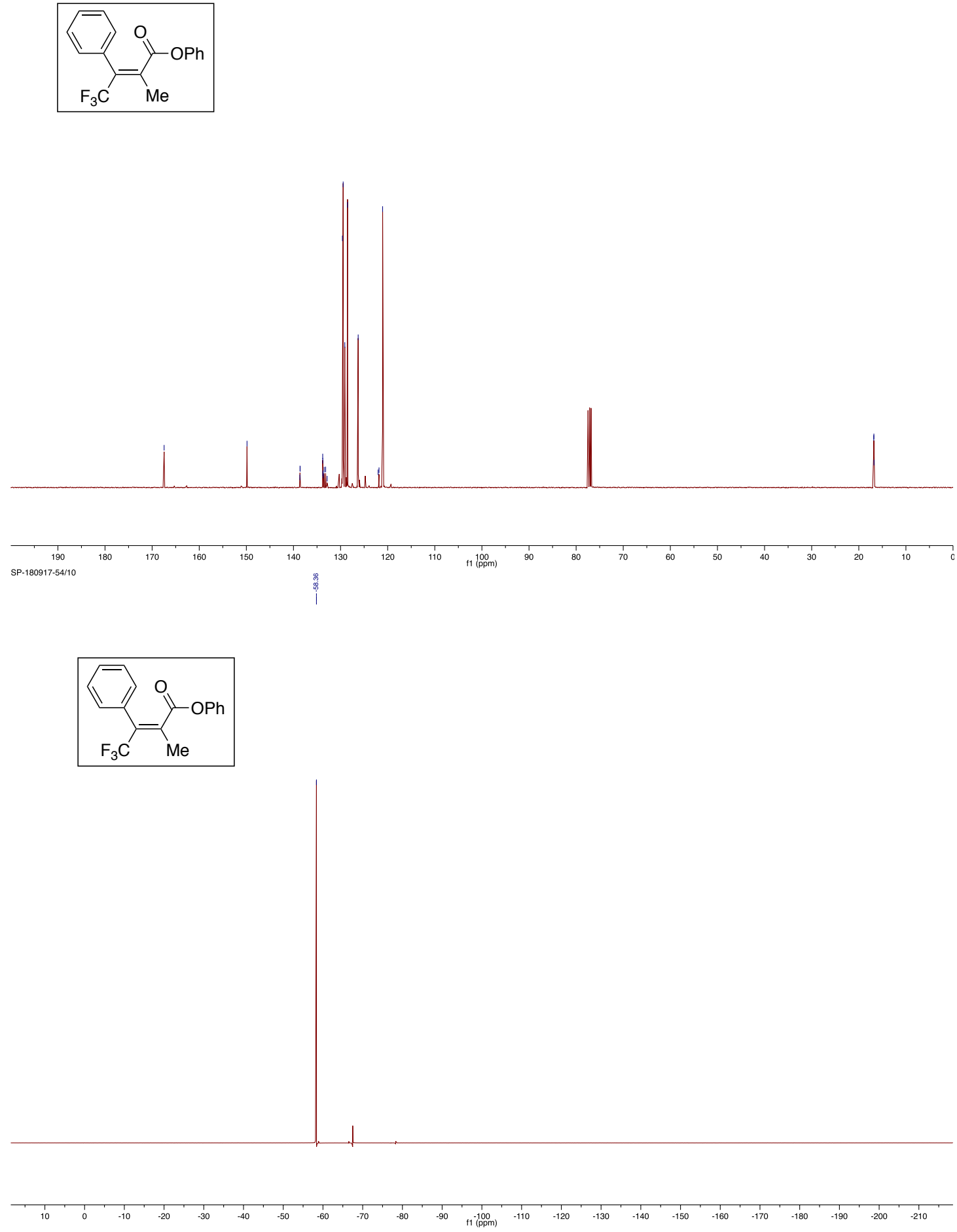

S-106 
SP-180916-80/10

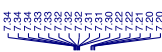

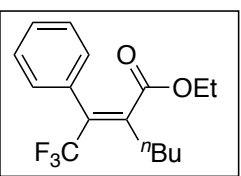

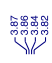
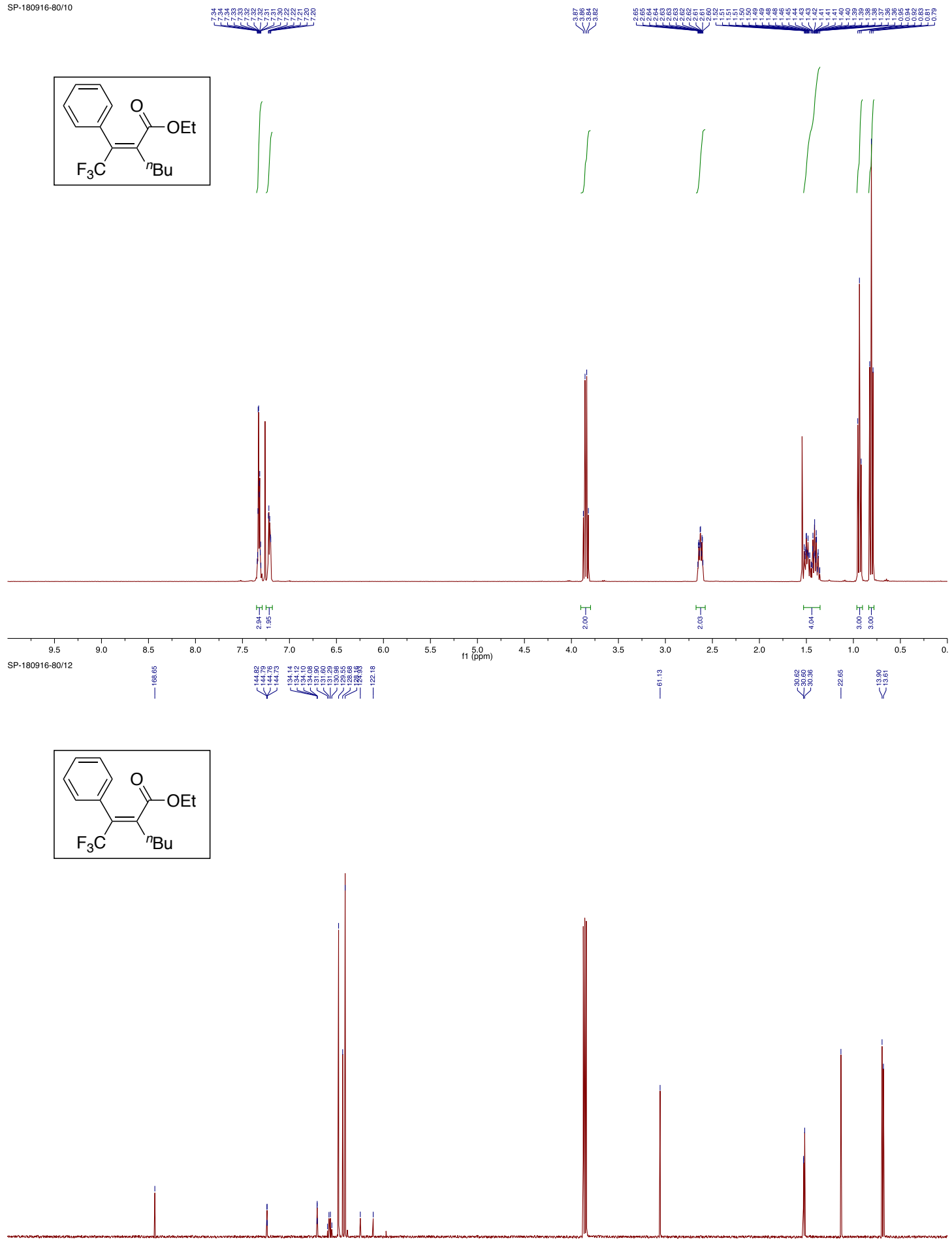

S-107 


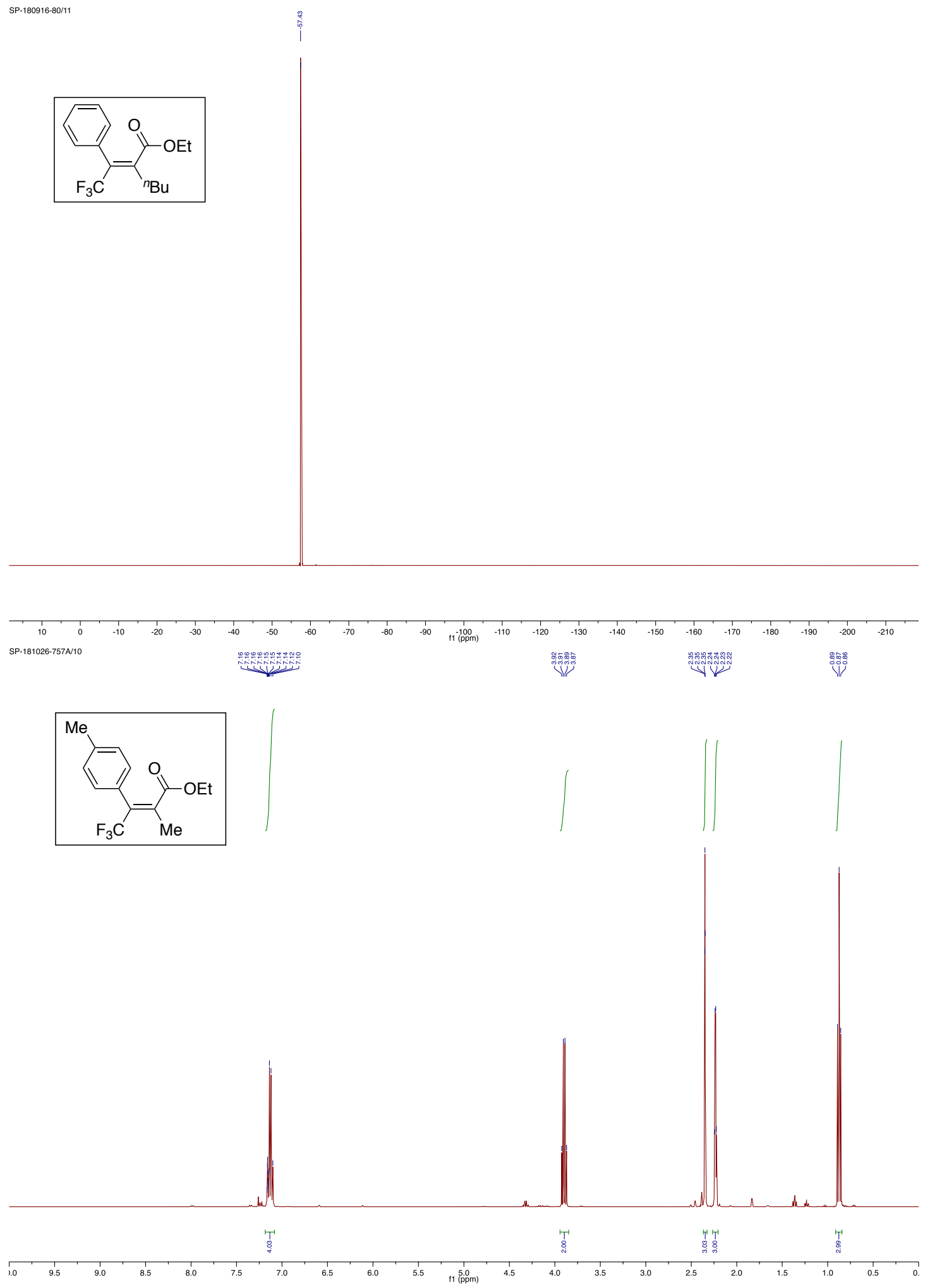

S-108 

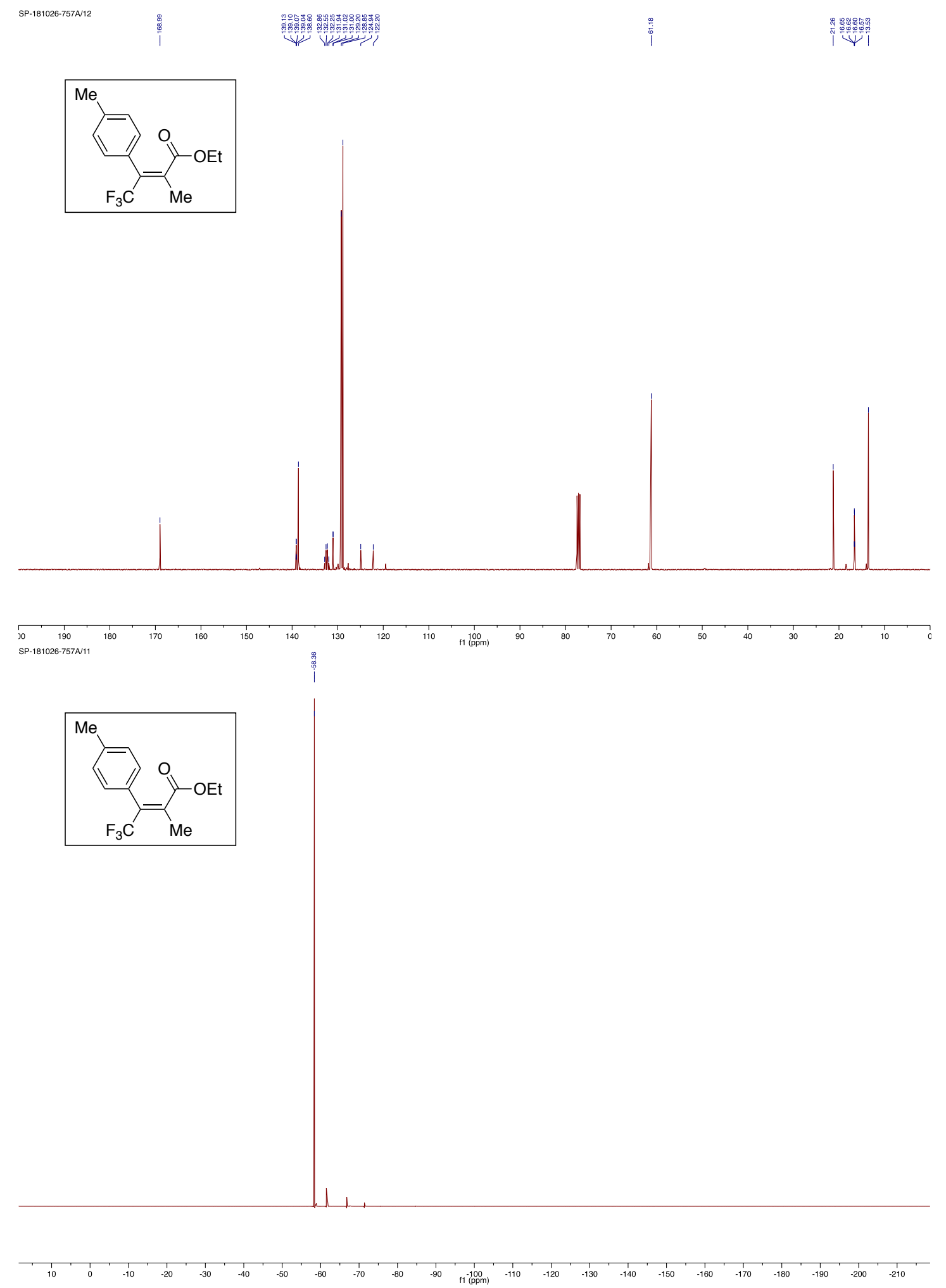

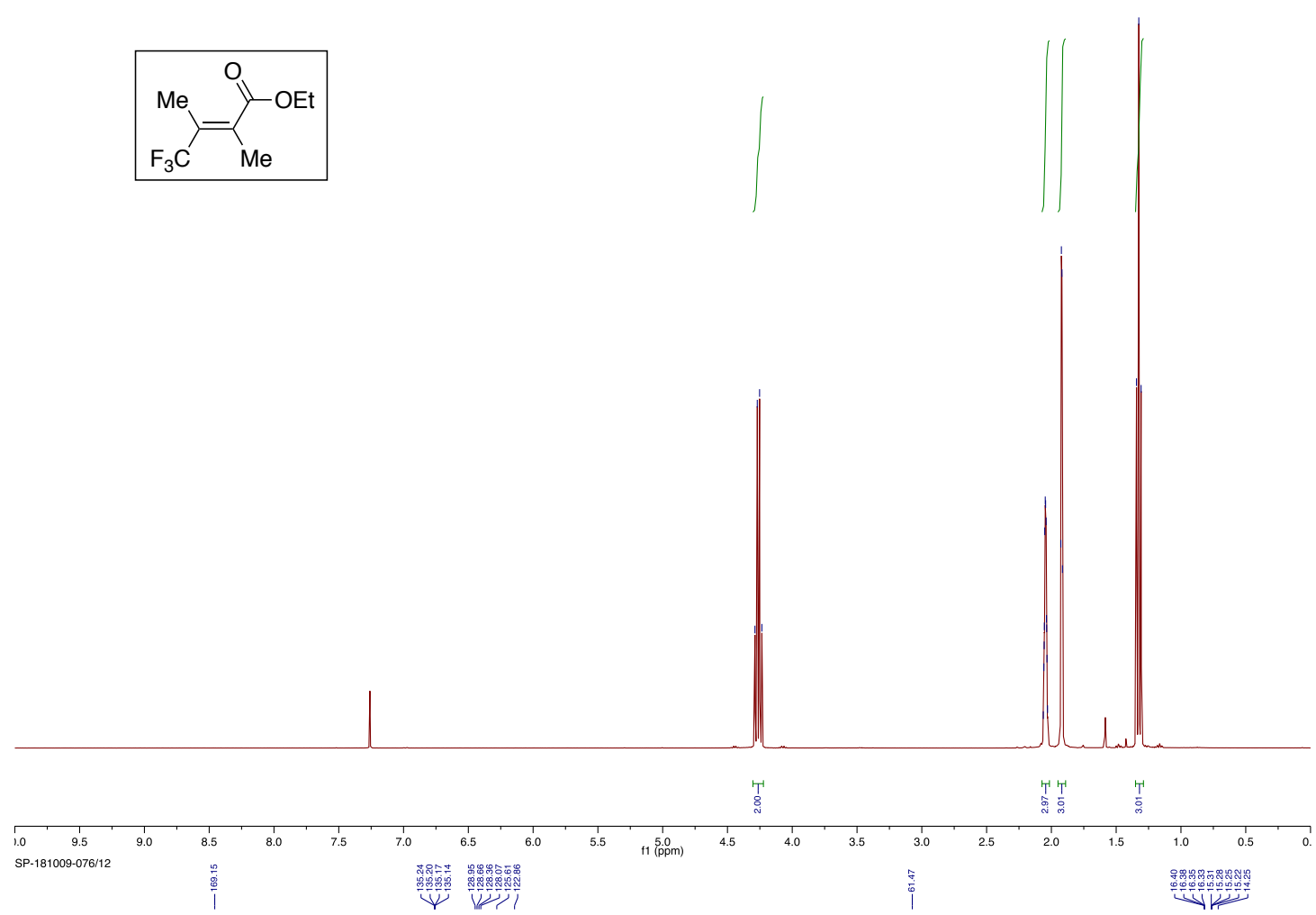

$\underset{\mathrm{F}_{3} \mathrm{C}}{\mathrm{Me}}=\mathrm{Me}_{\mathrm{OEt}}^{\mathrm{O}}$

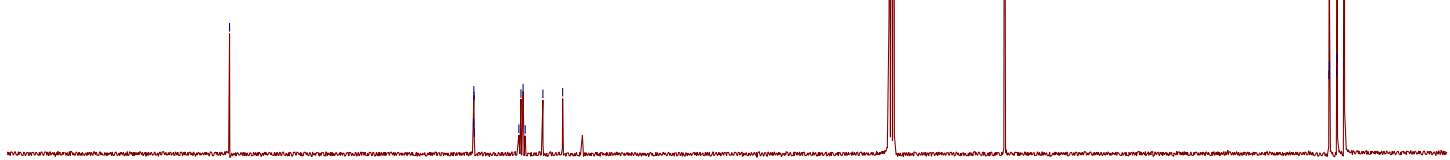



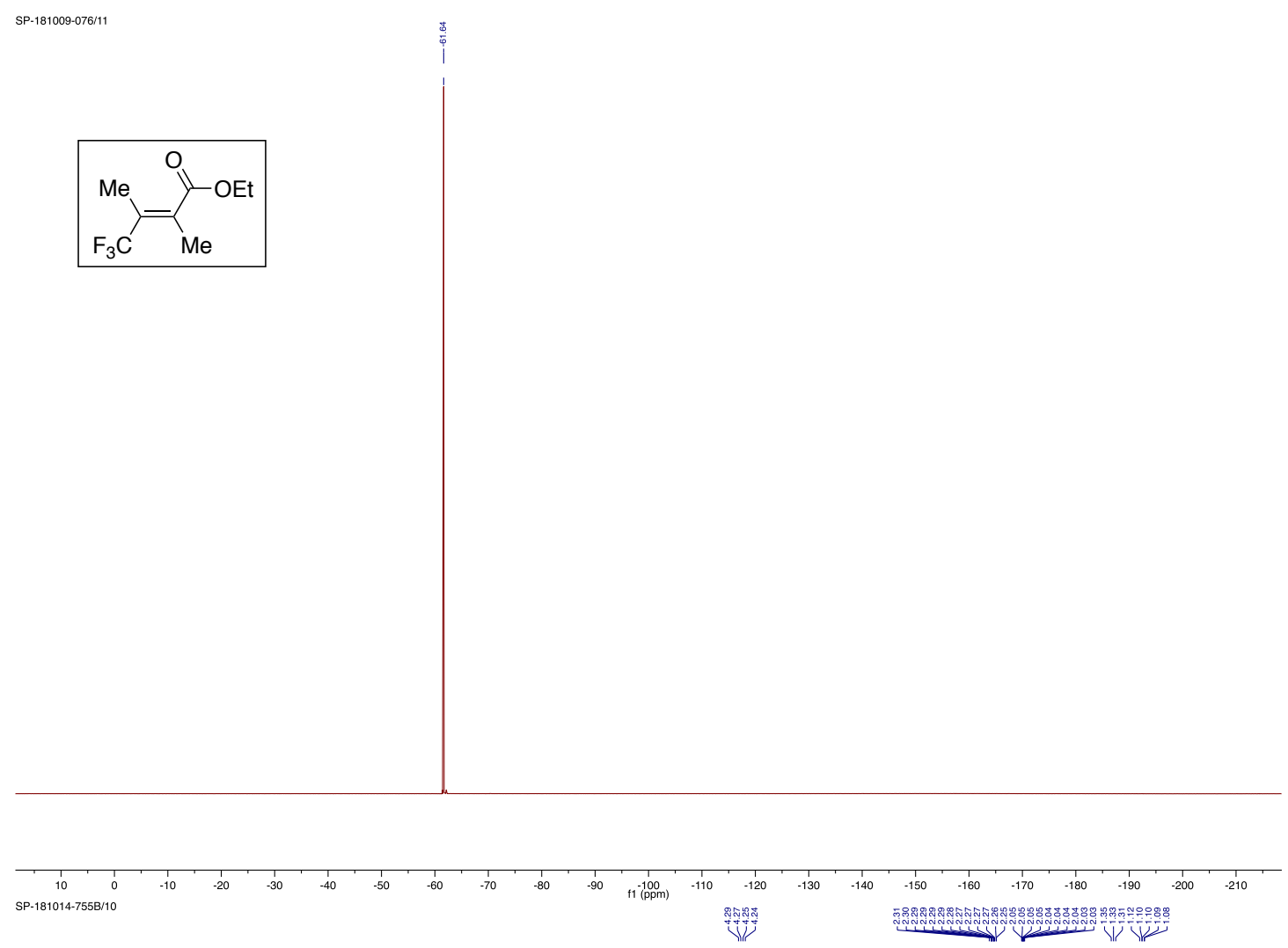

$\mathrm{F}_{3} \mathrm{Ct}$

स W W V V

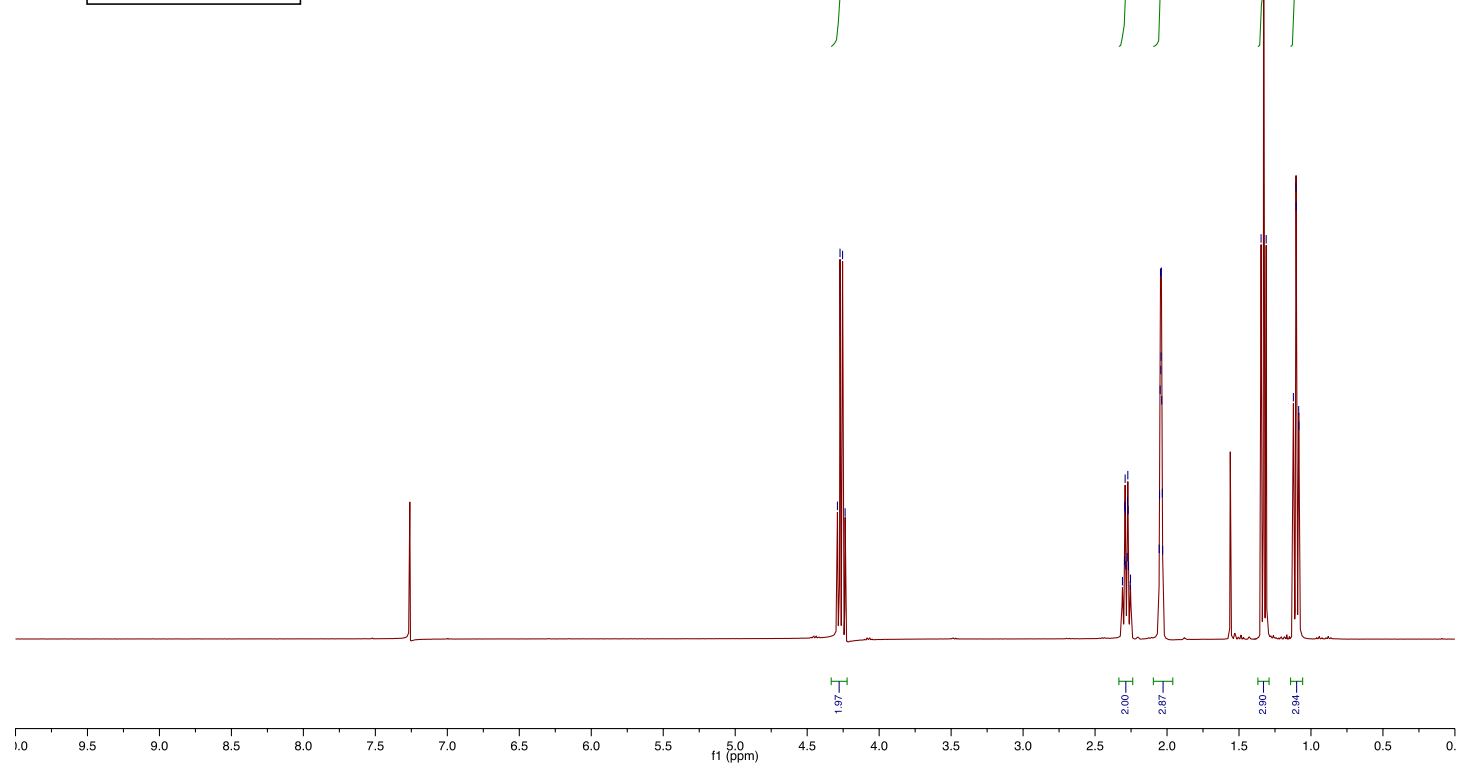

S-111 

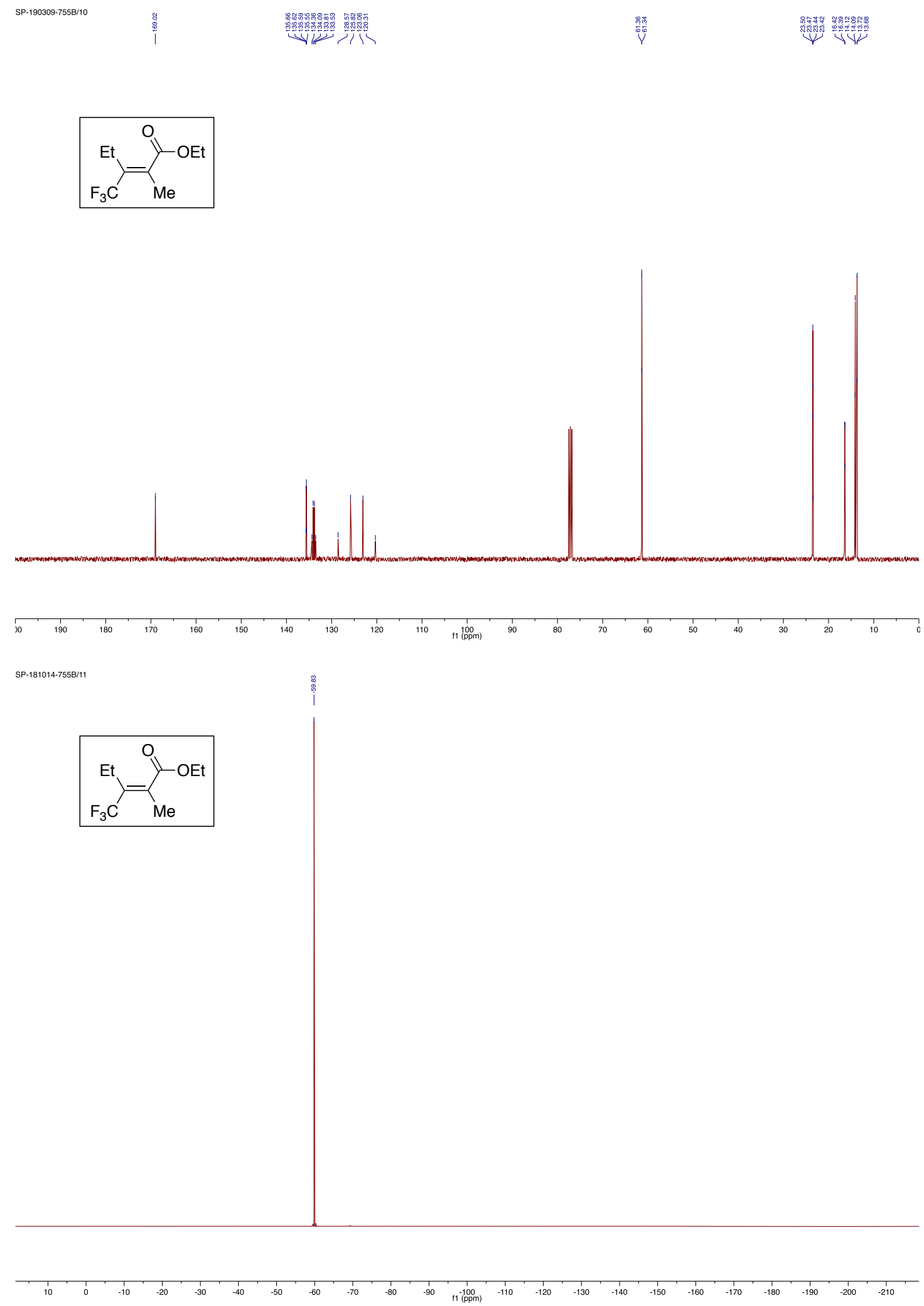

S-112 

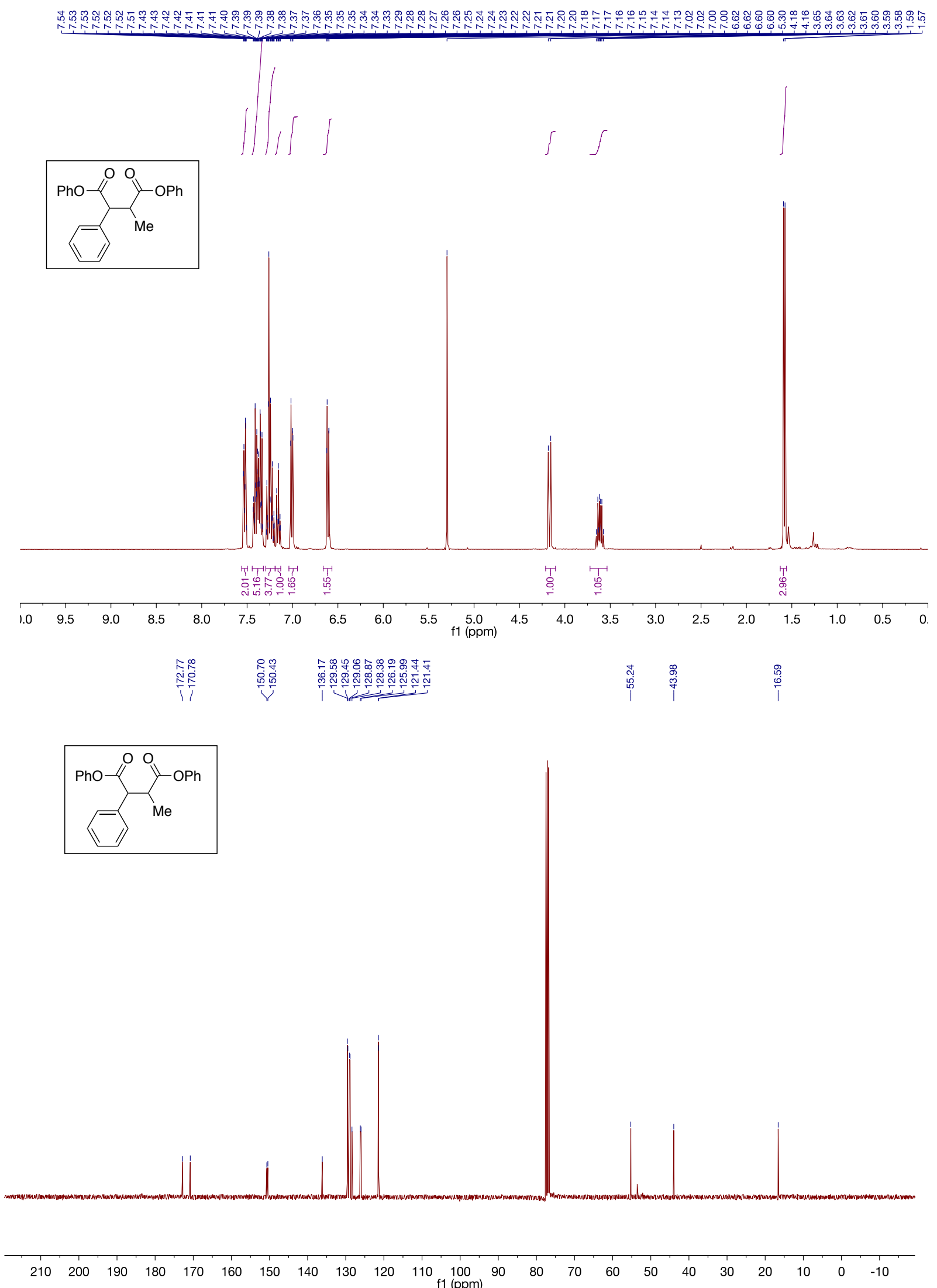

S-113 


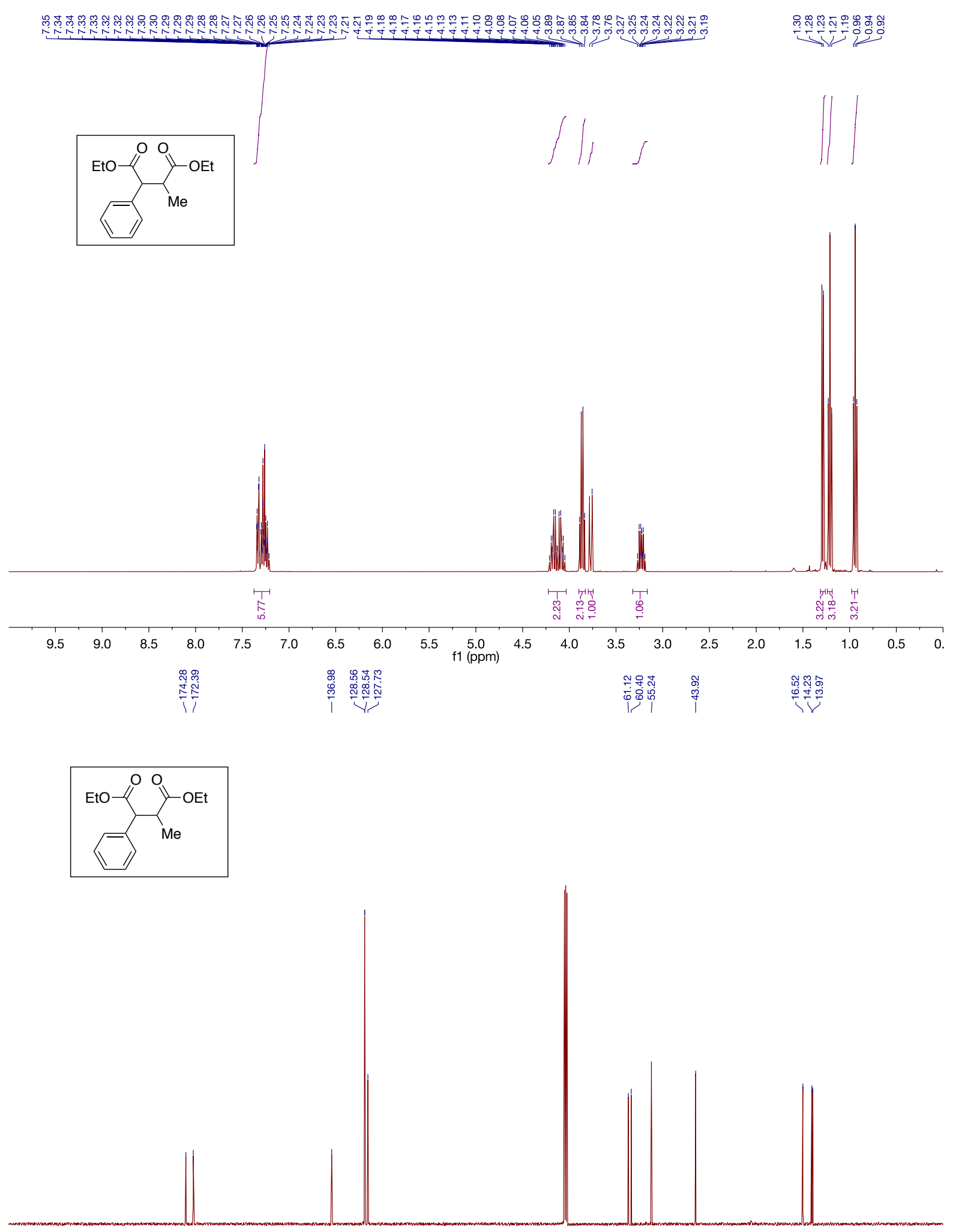

$\begin{array}{llllllllllllllllllllllllll}210 & 200 & 190 & 180 & 170 & 160 & 150 & 140 & 130 & 120 & 110 & 100 & 90 & 80 & 70 & 60 & 50 & 40 & 30 & 20 & 10 & 0 & -10 & 10\end{array}$ 


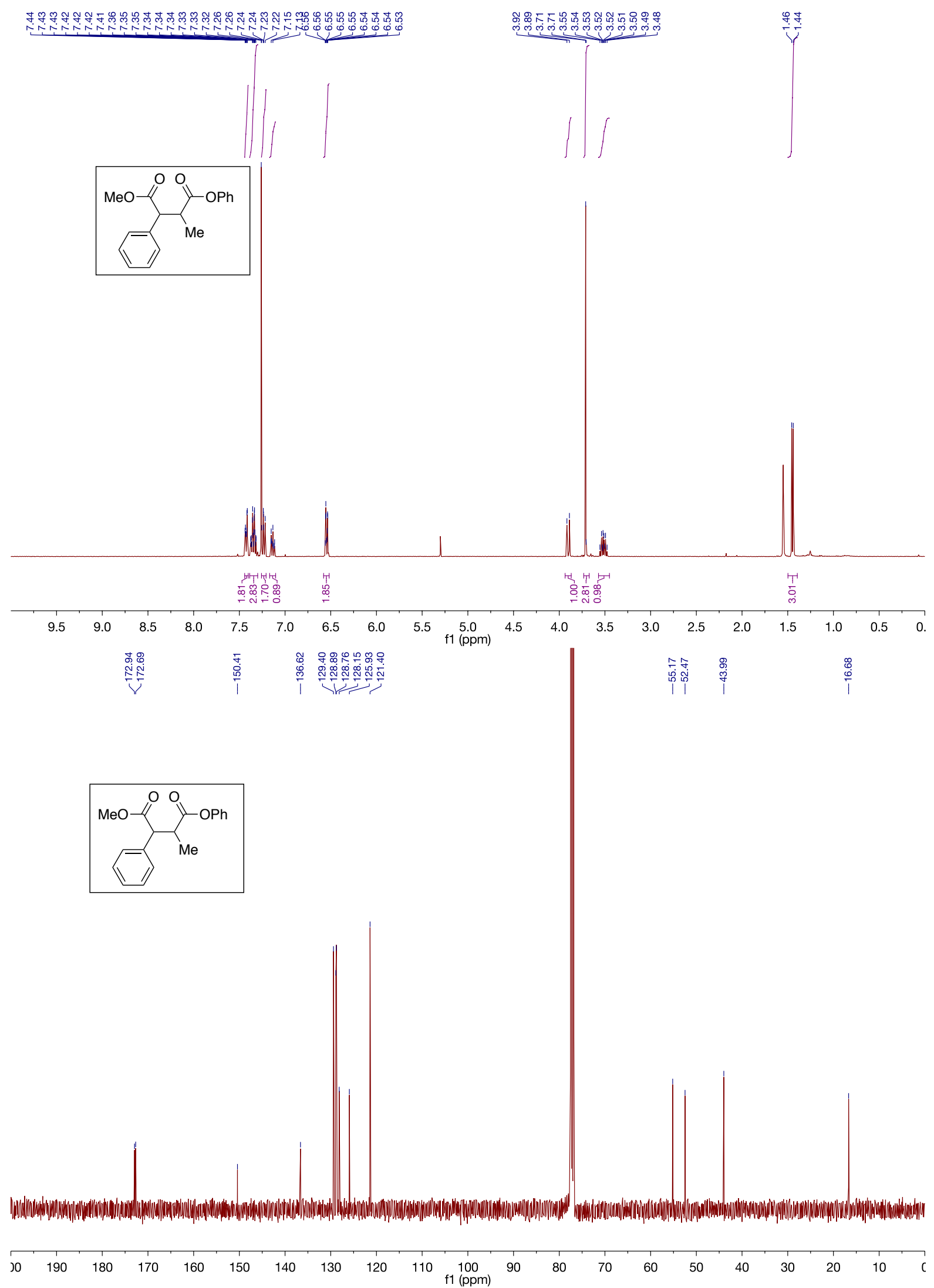

S-115 

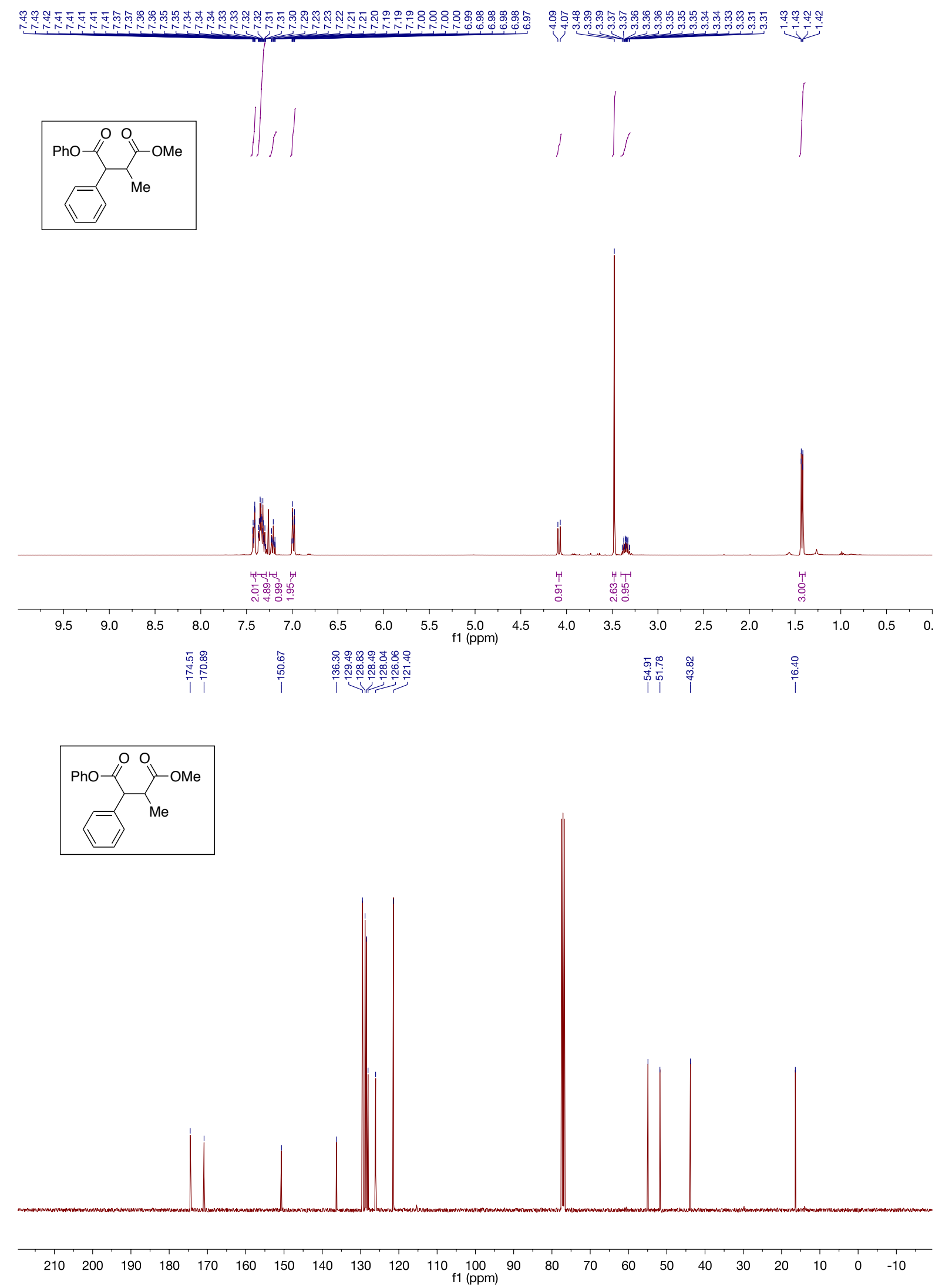

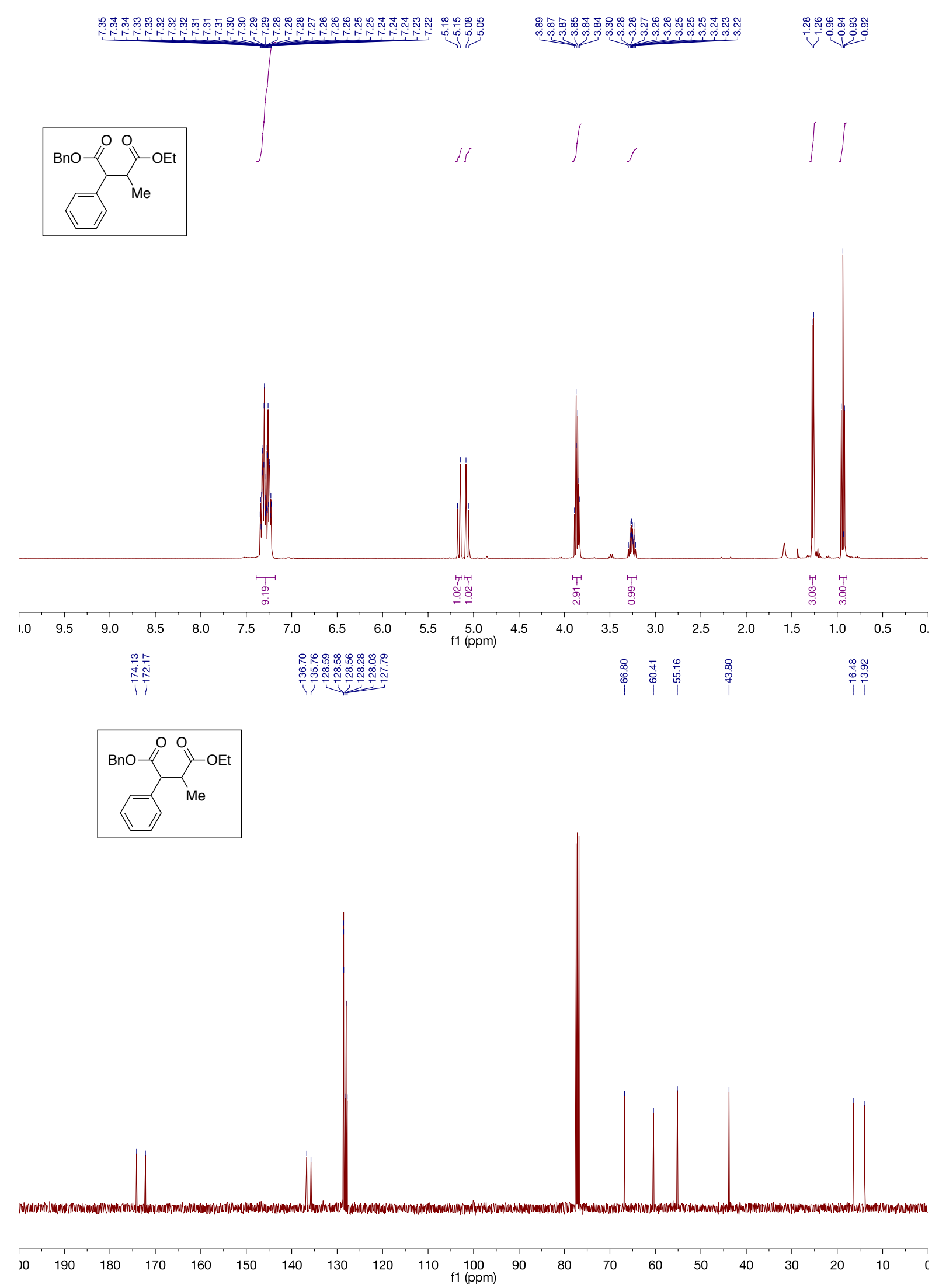


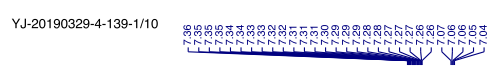

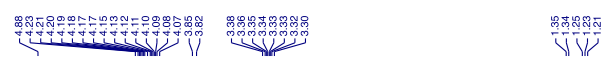
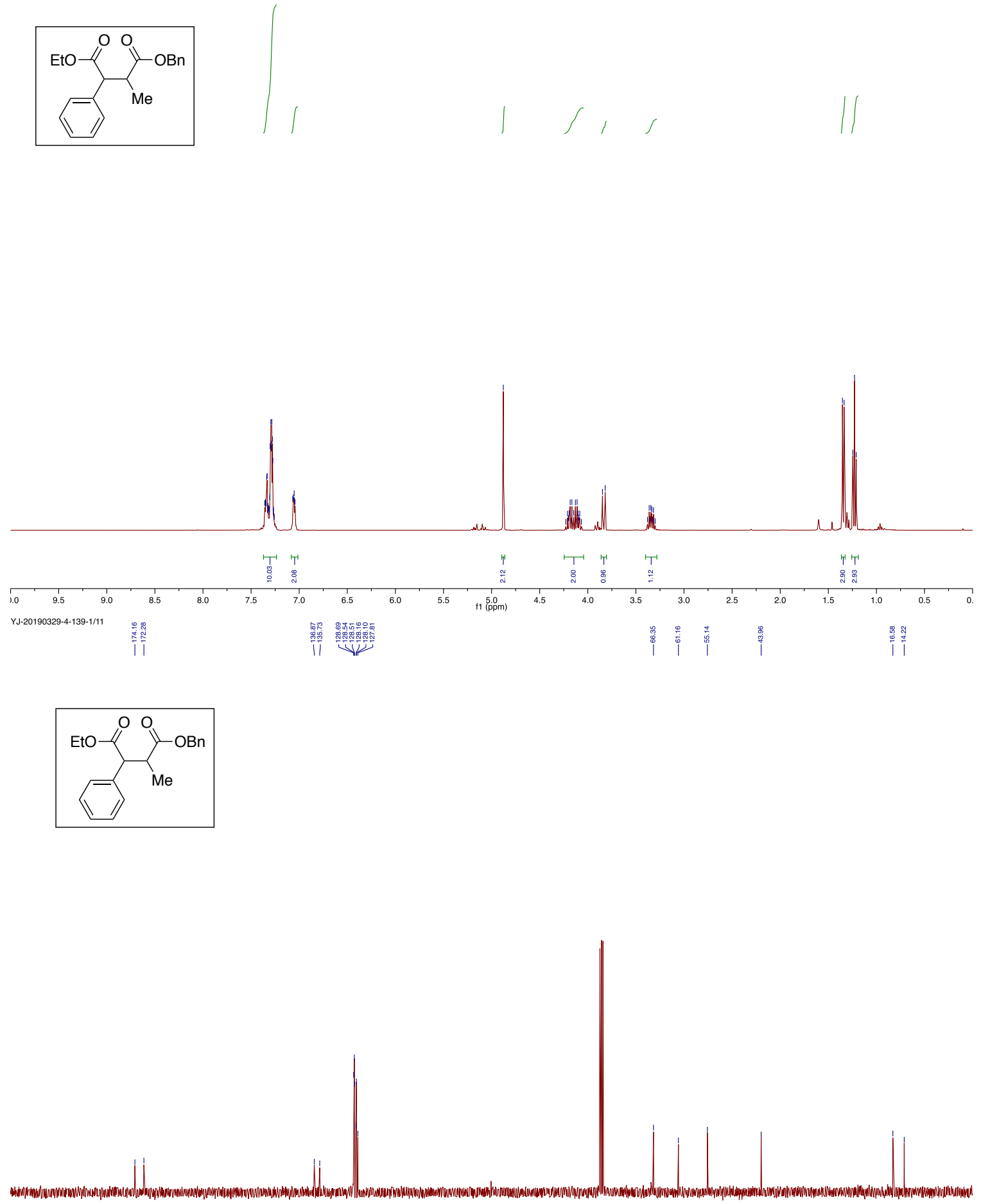
$\underbrace{.04}$
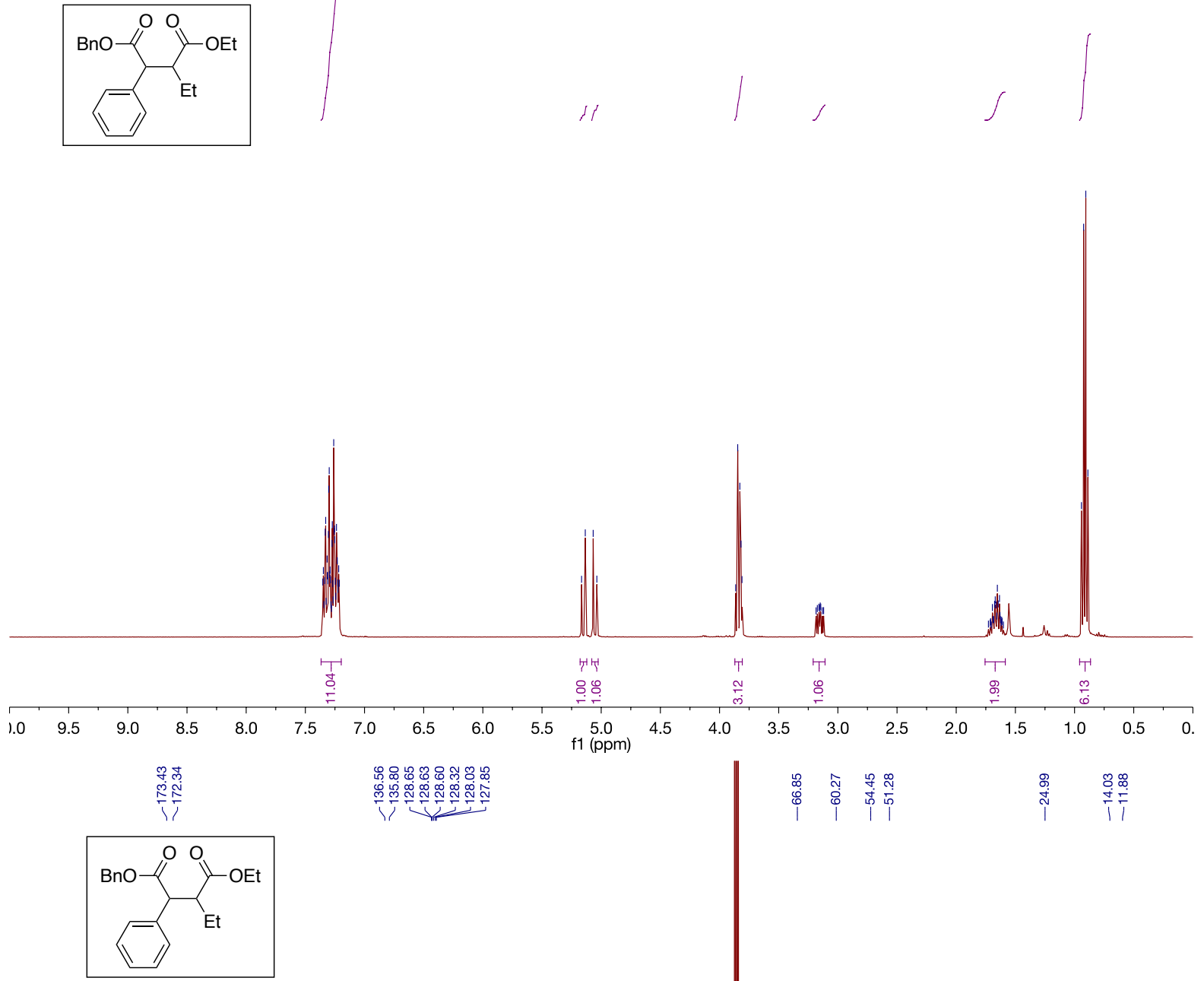

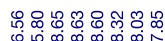

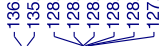

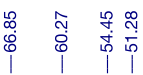

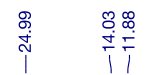
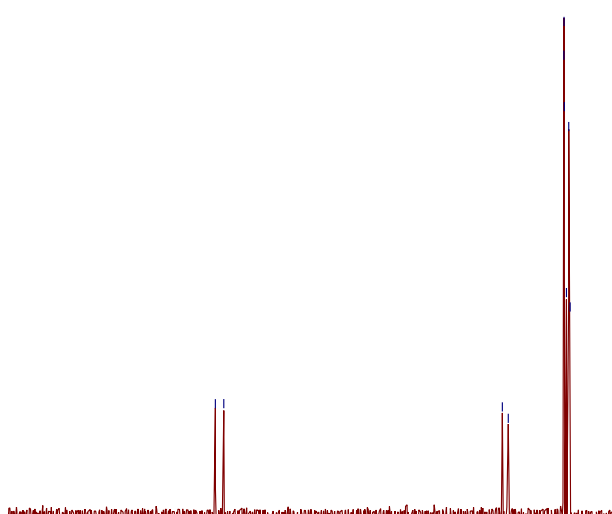

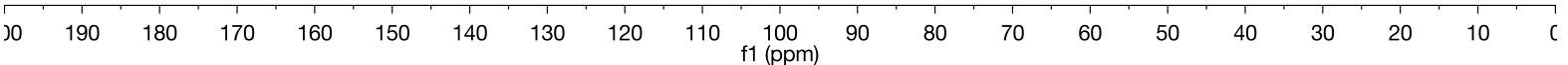



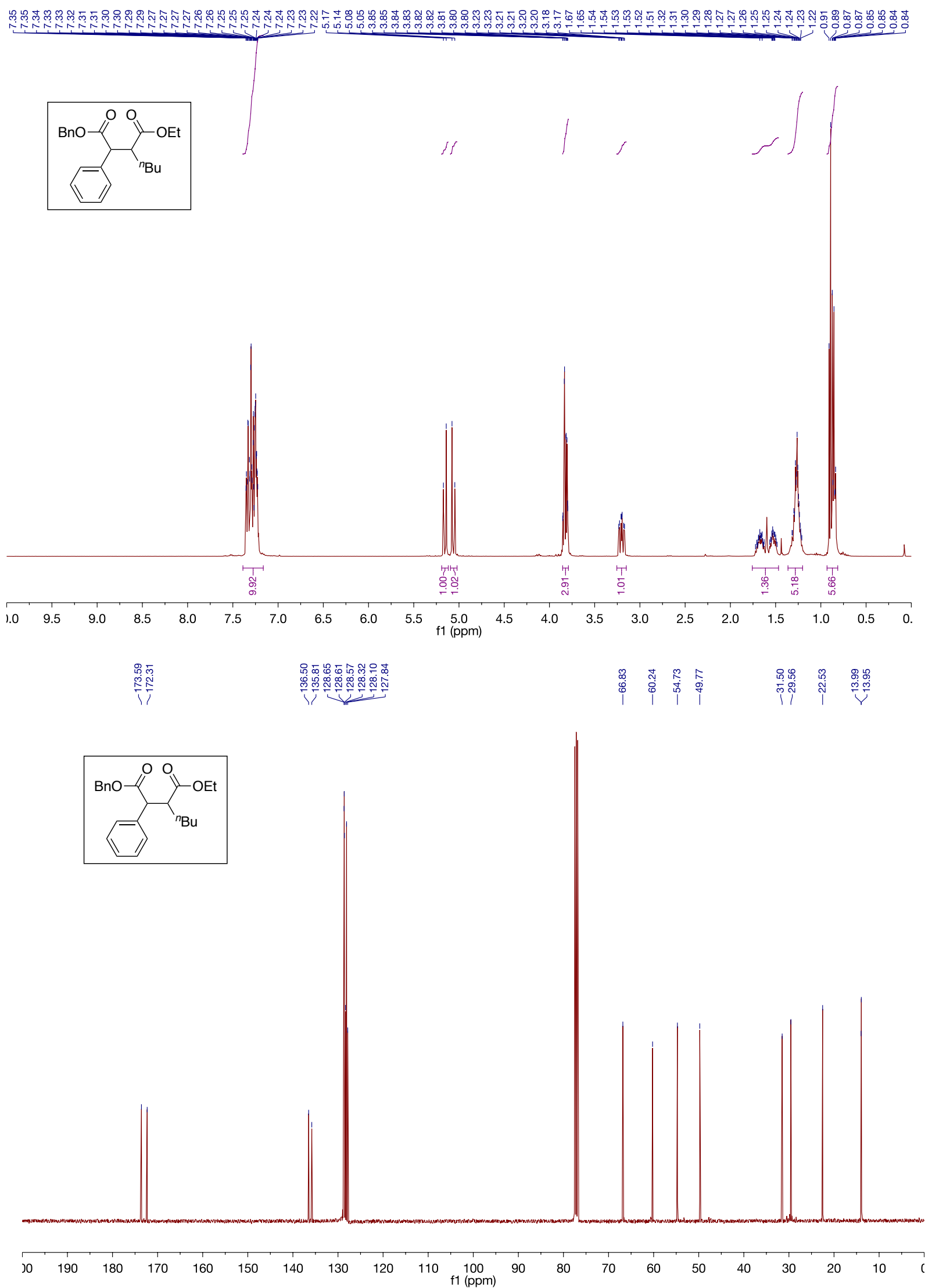


COCF
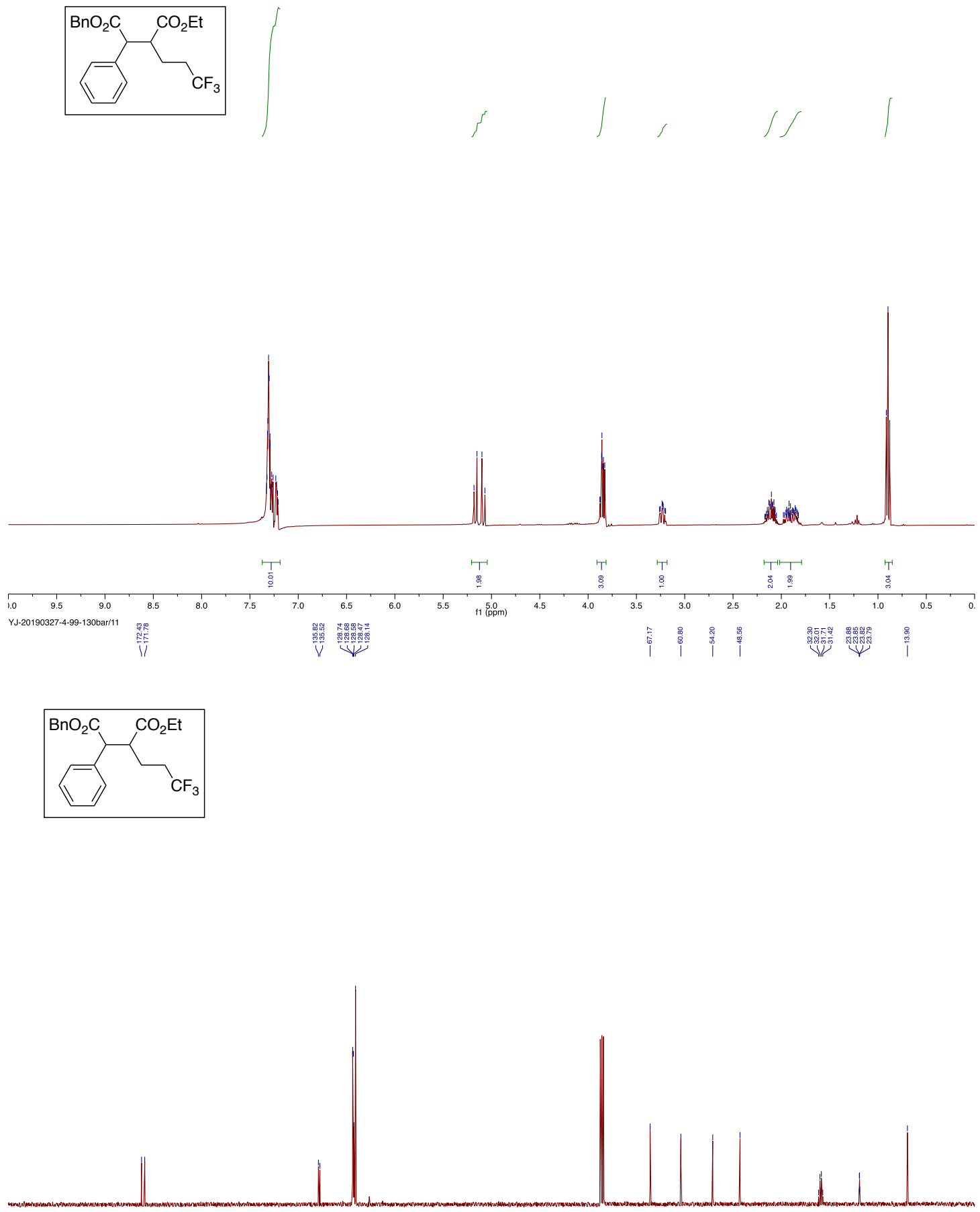


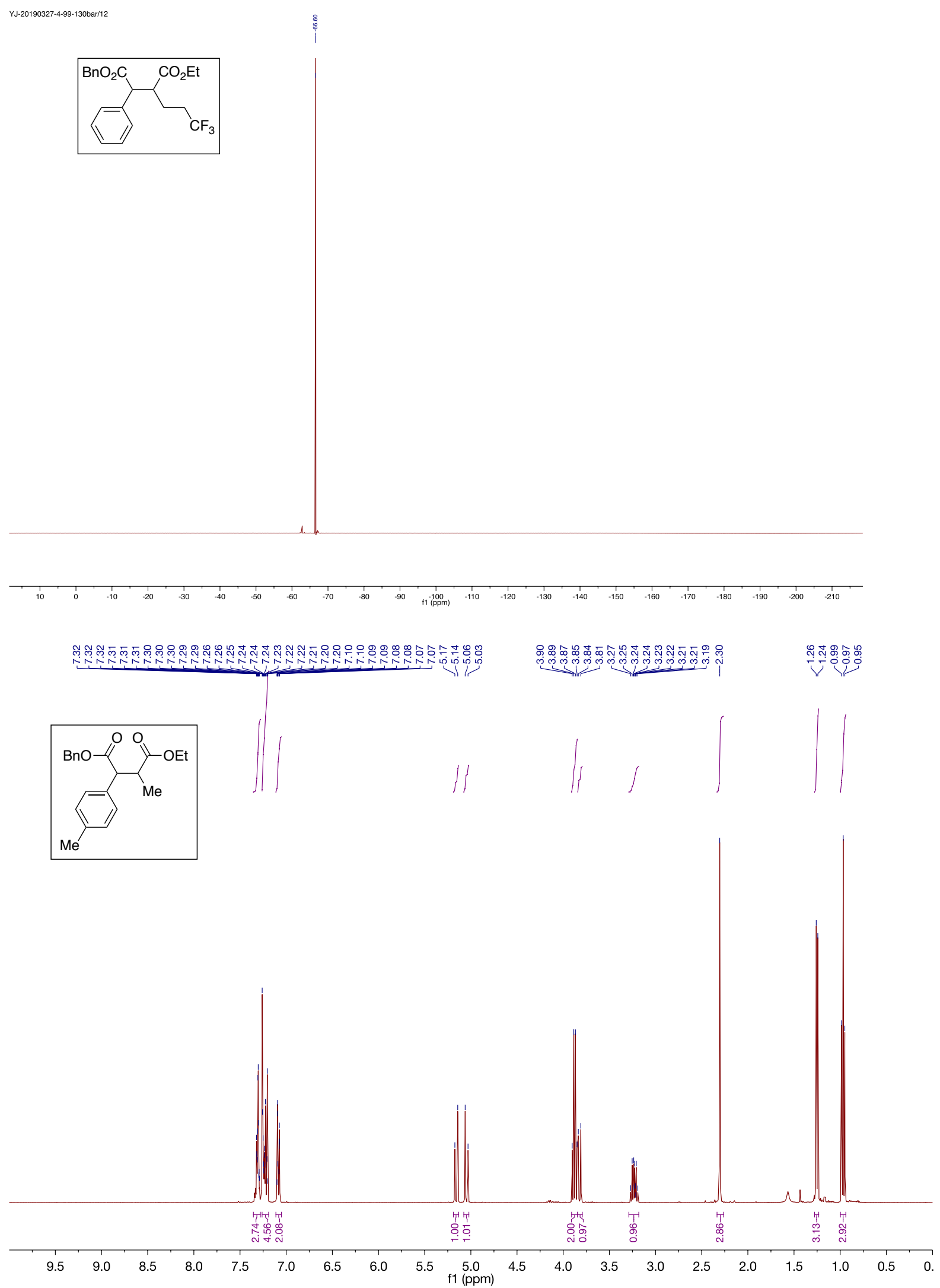

S-122 


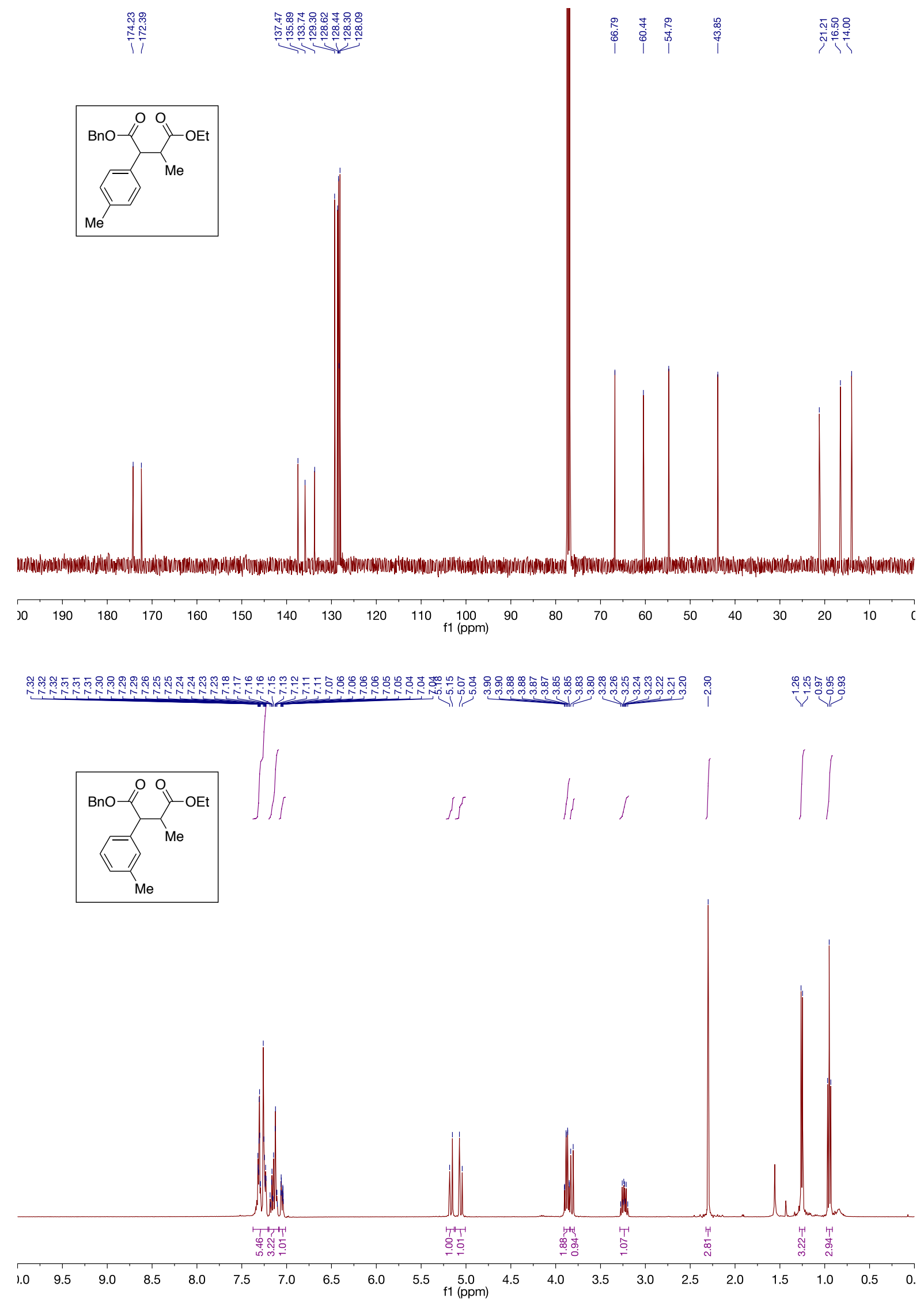

S-123 


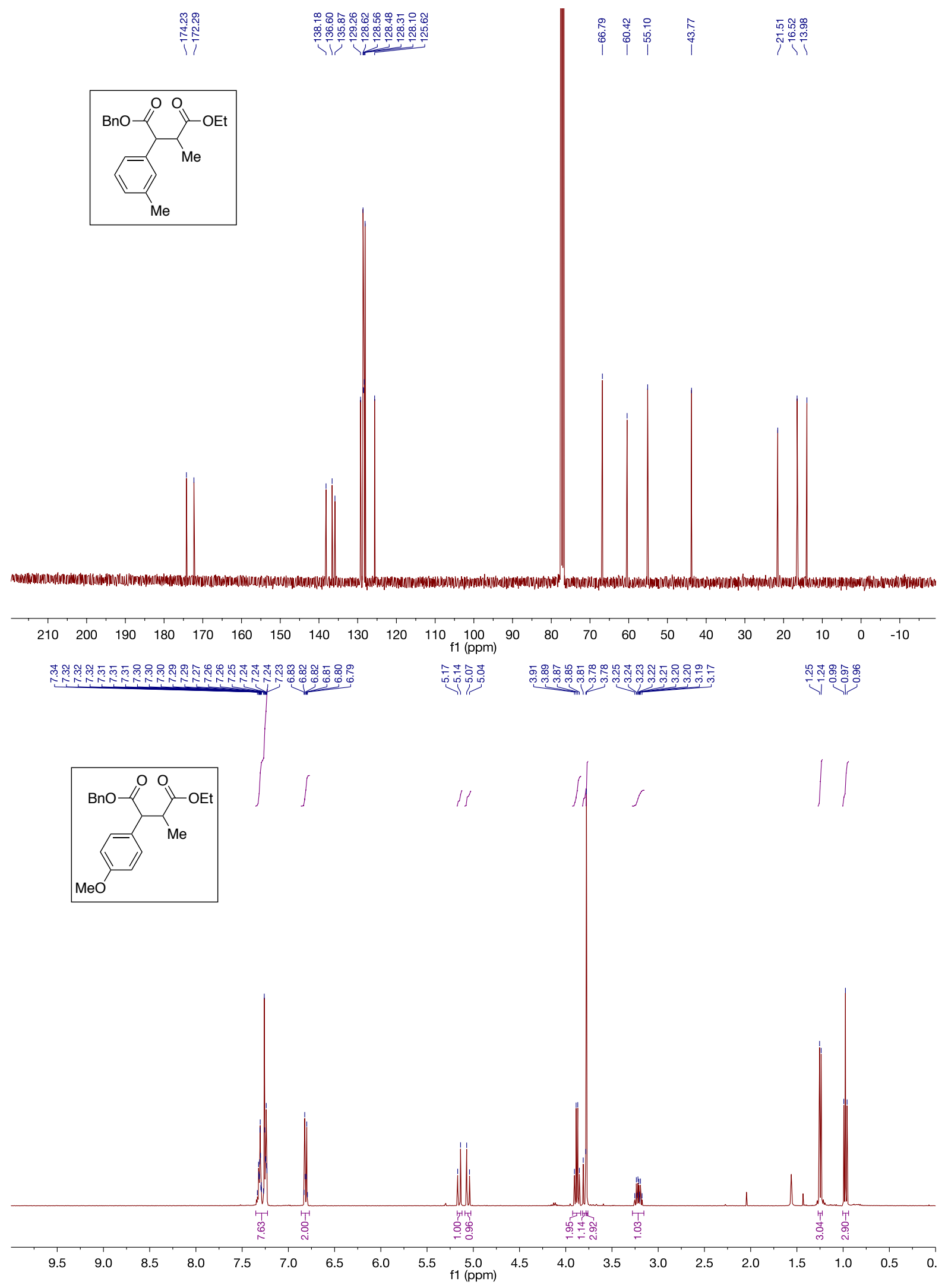




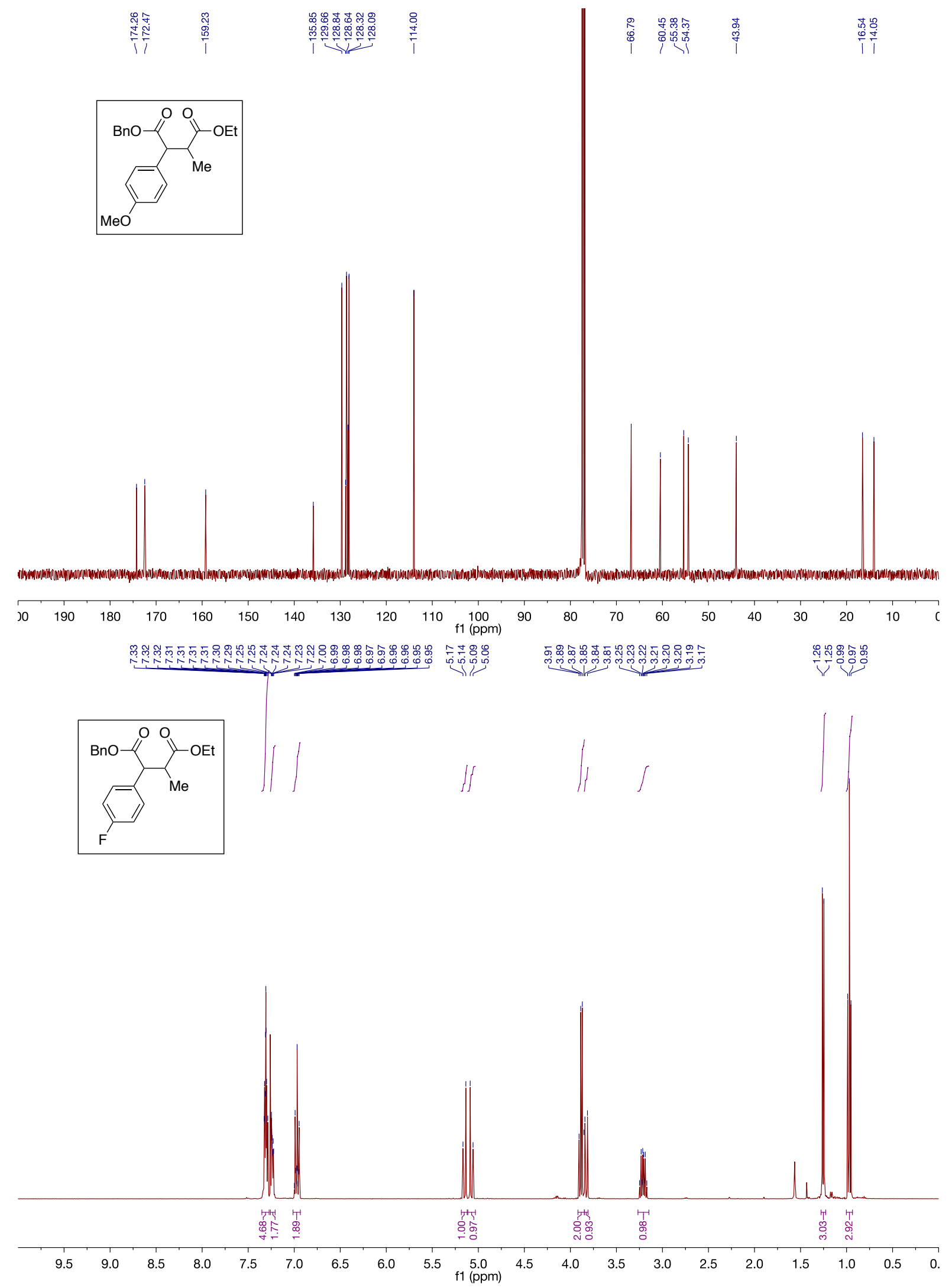



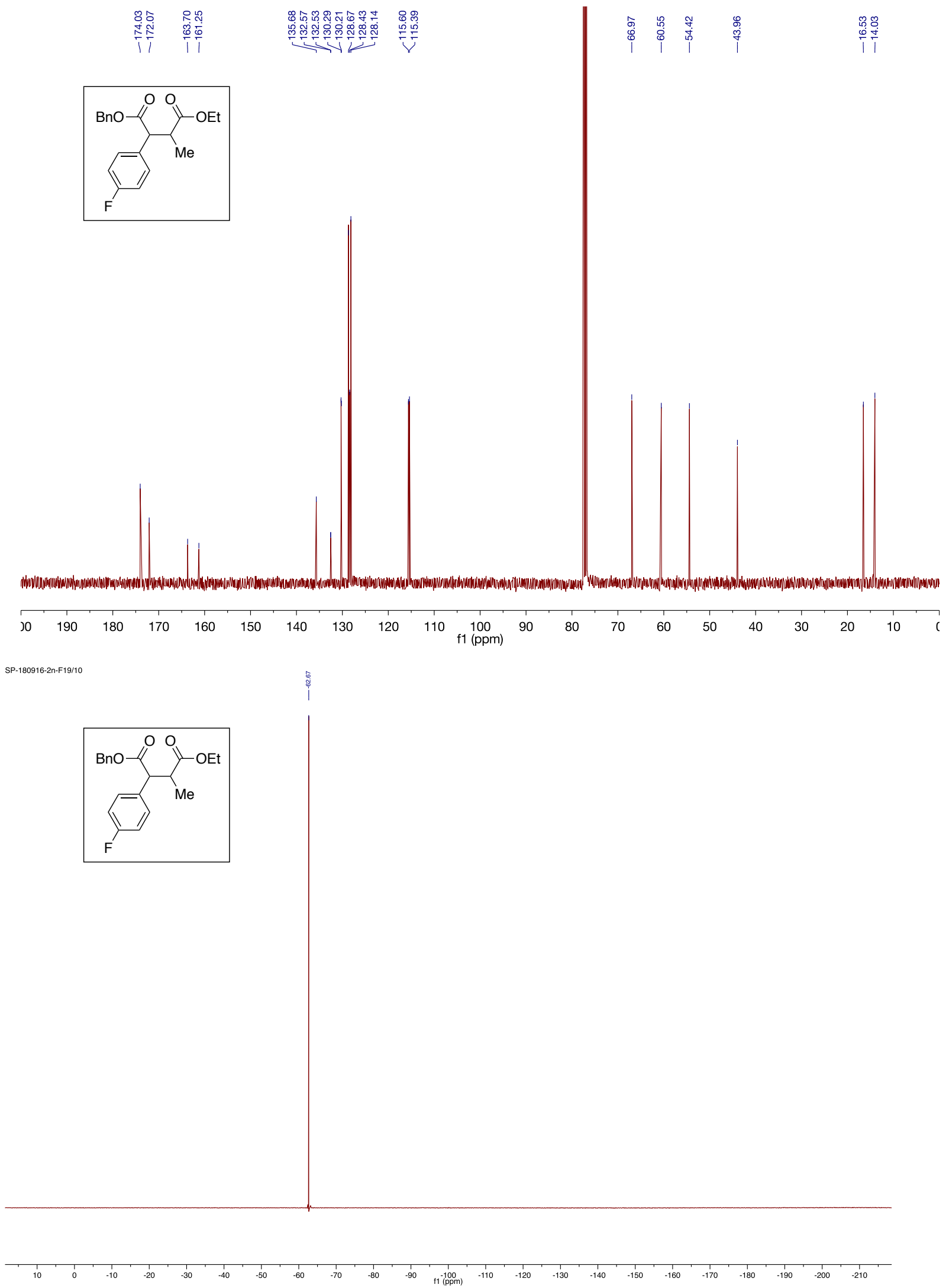

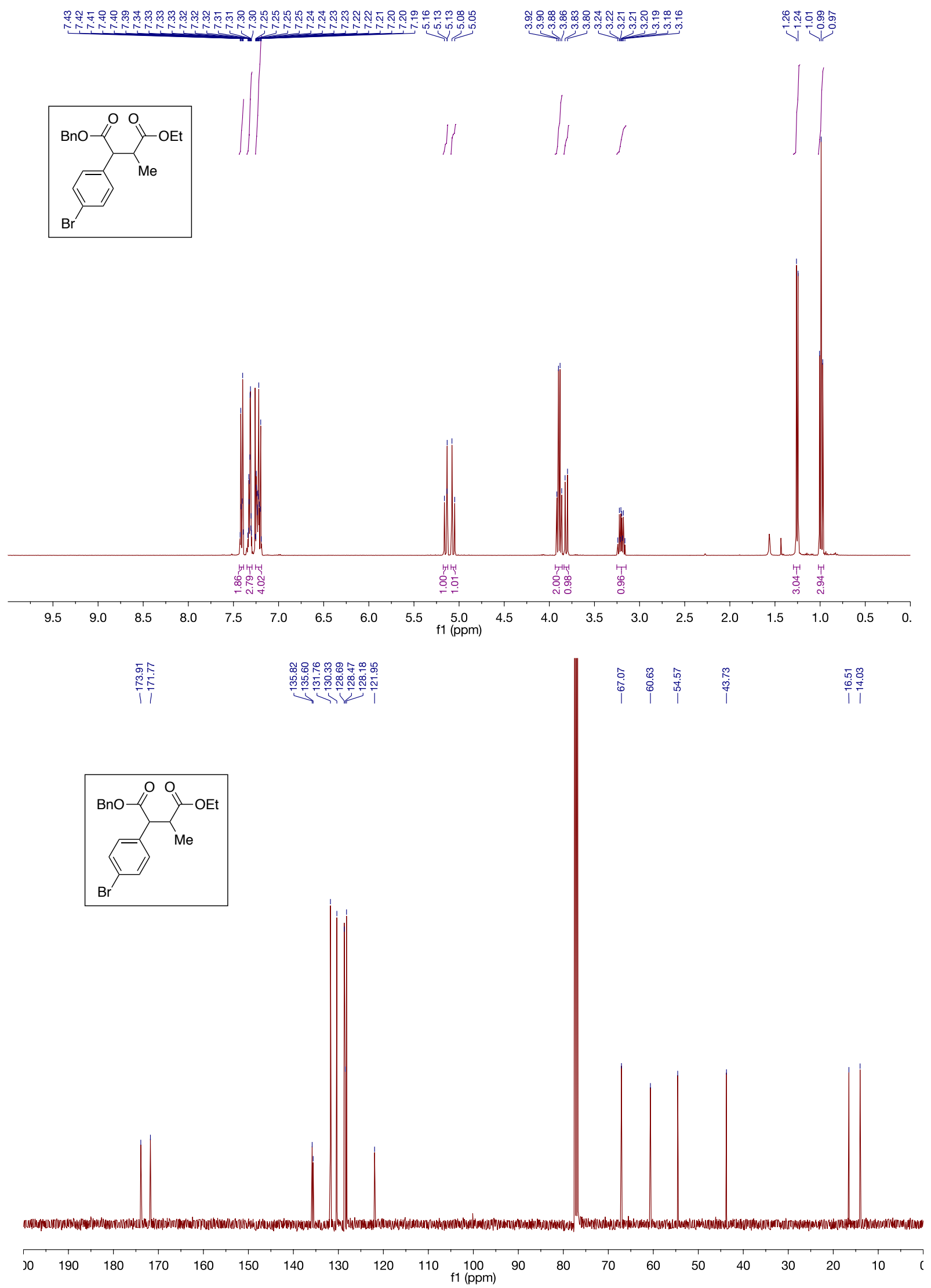


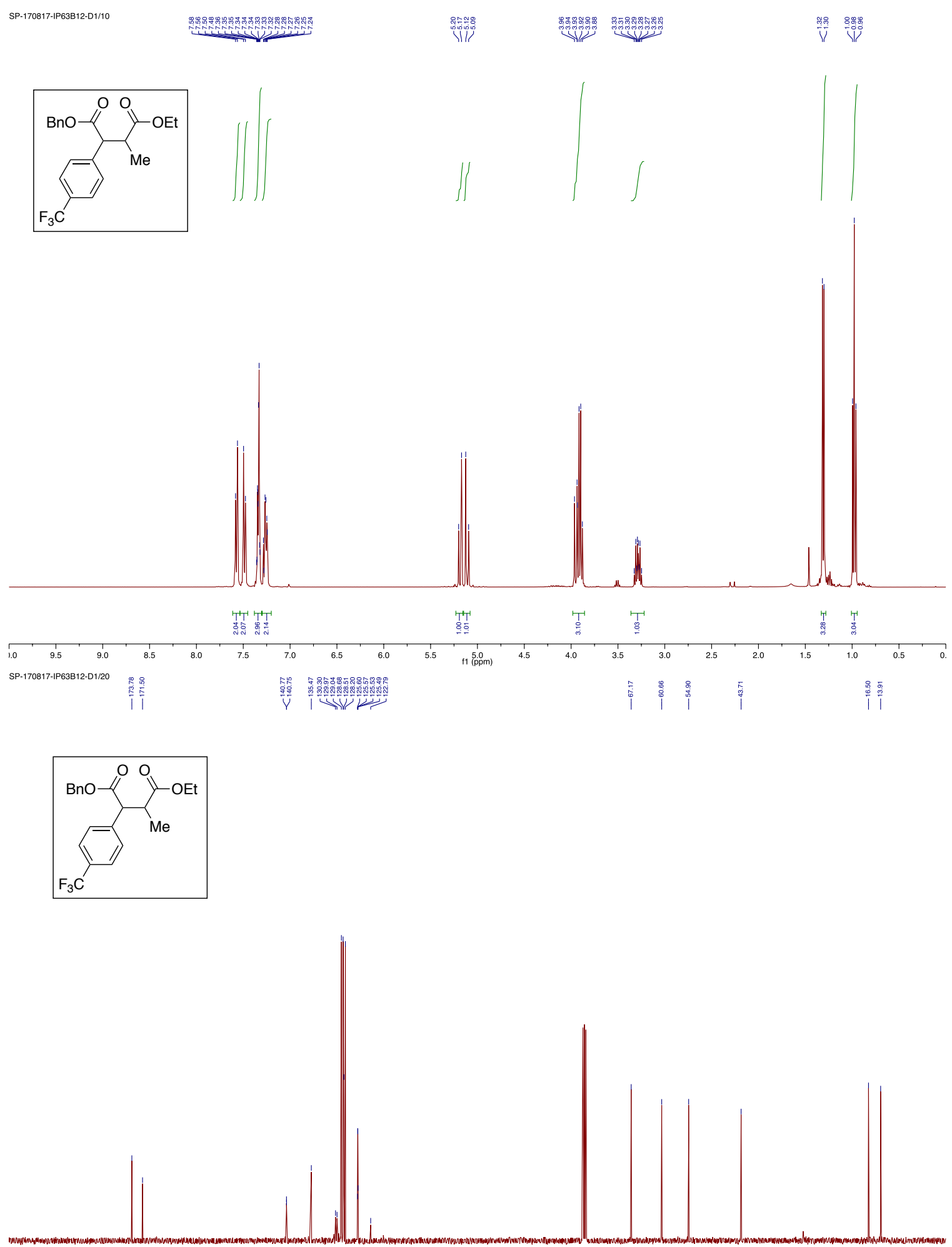

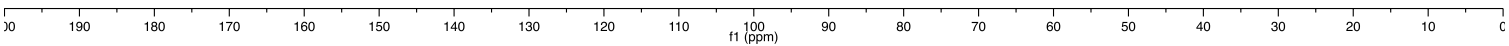


SP-180916-2P-F19/11
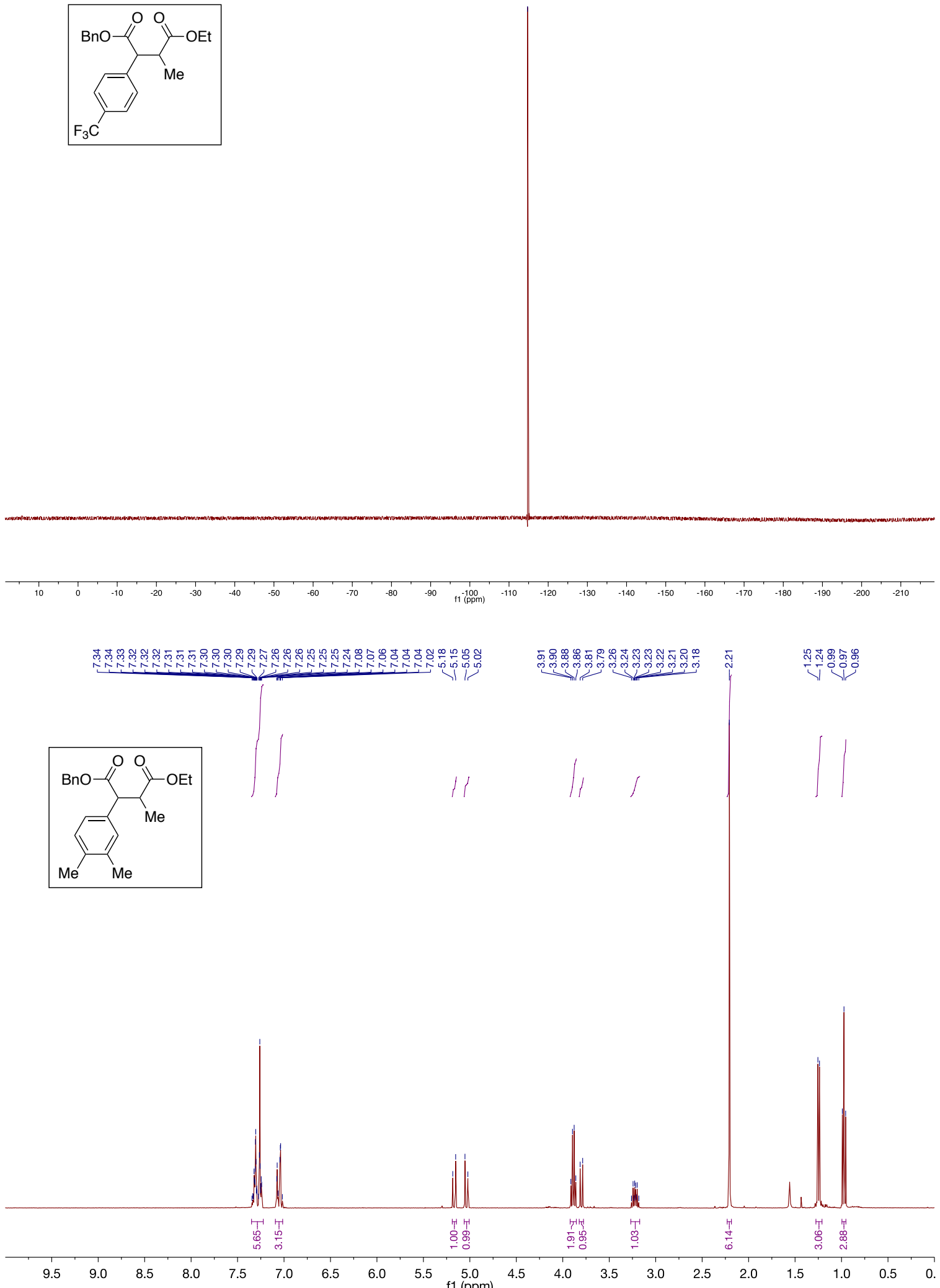


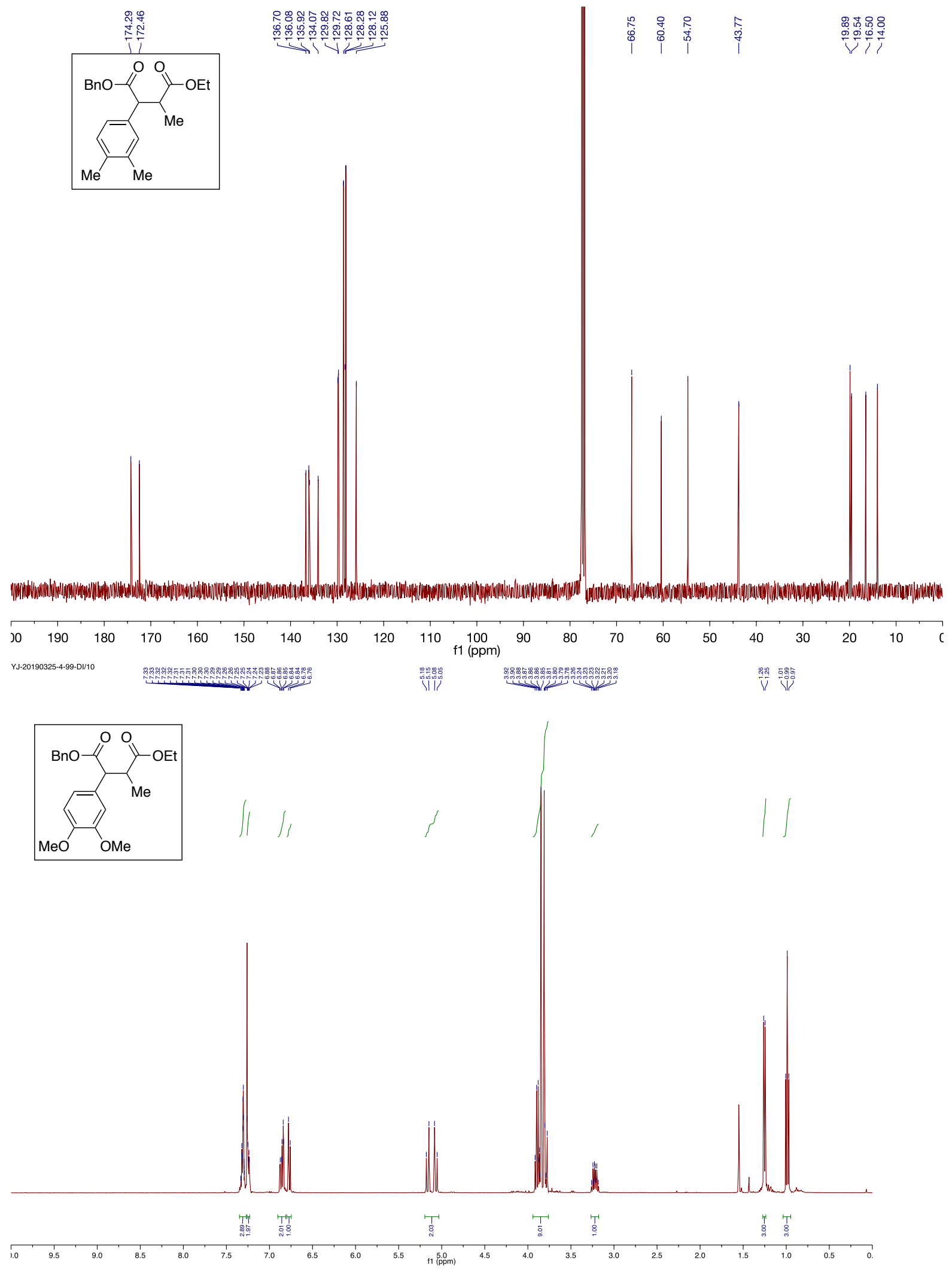



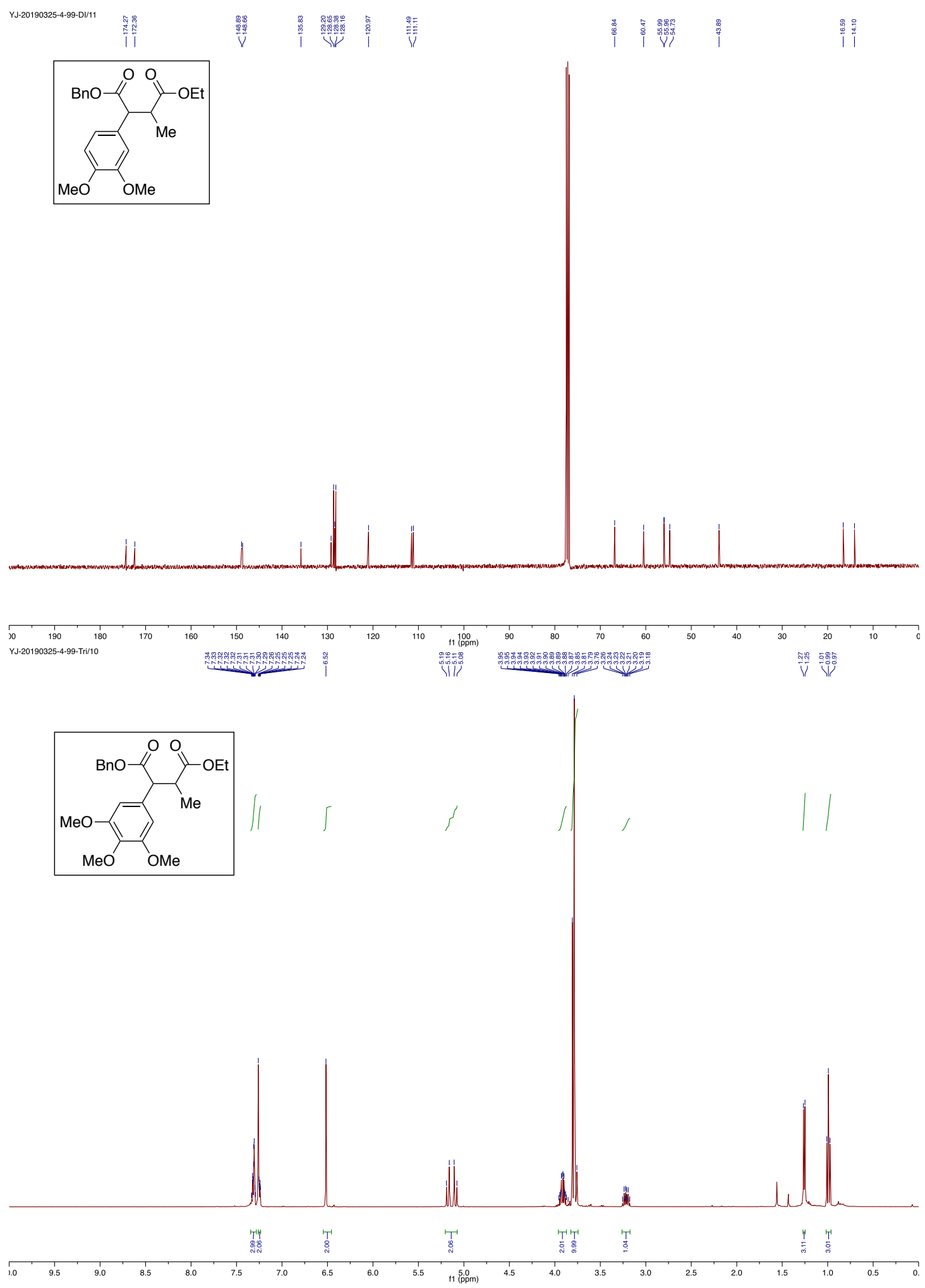

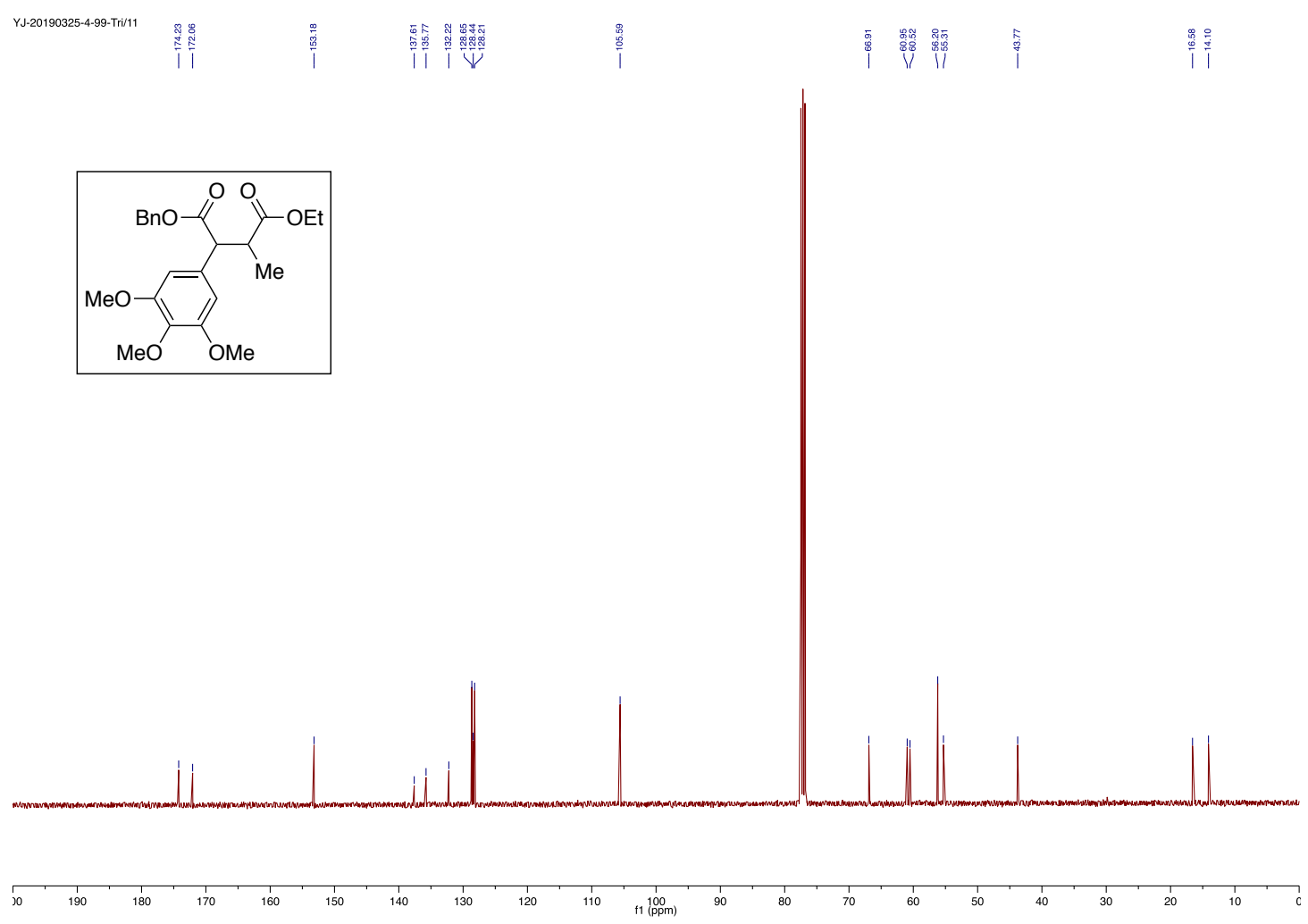

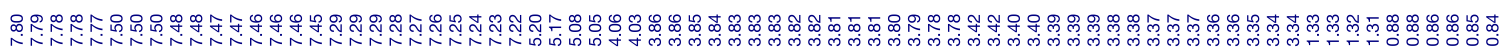
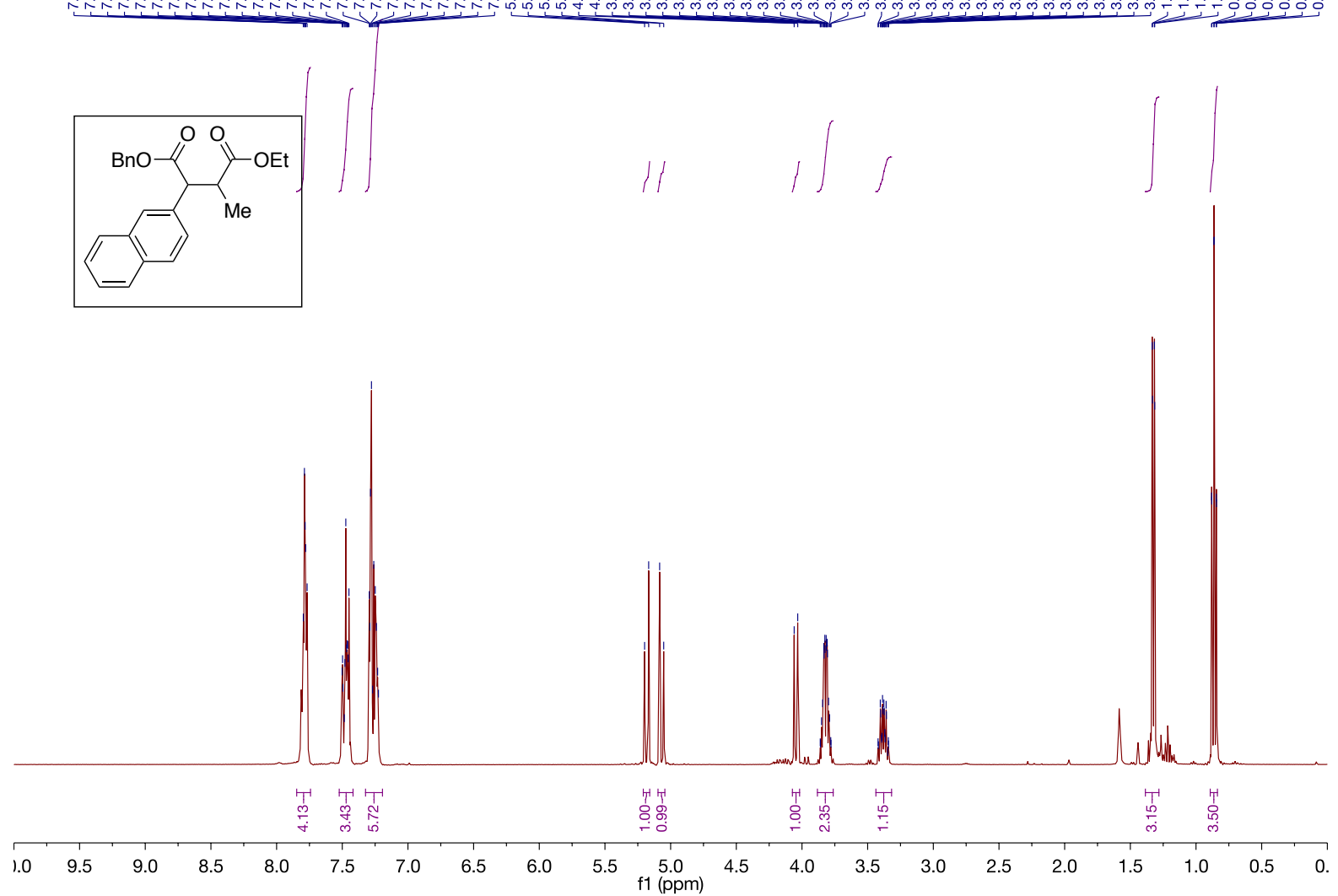

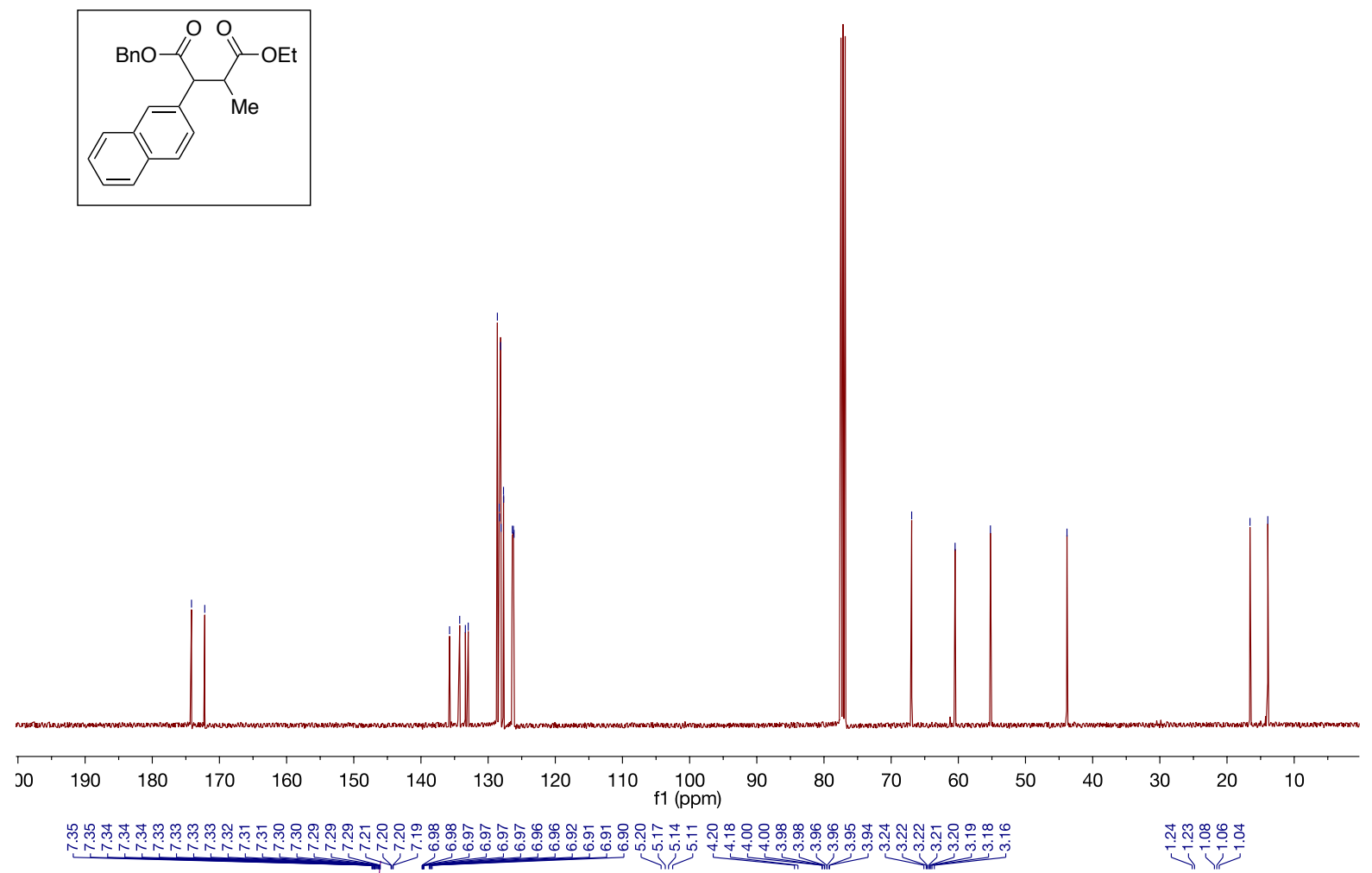

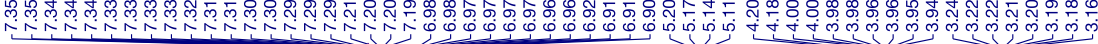

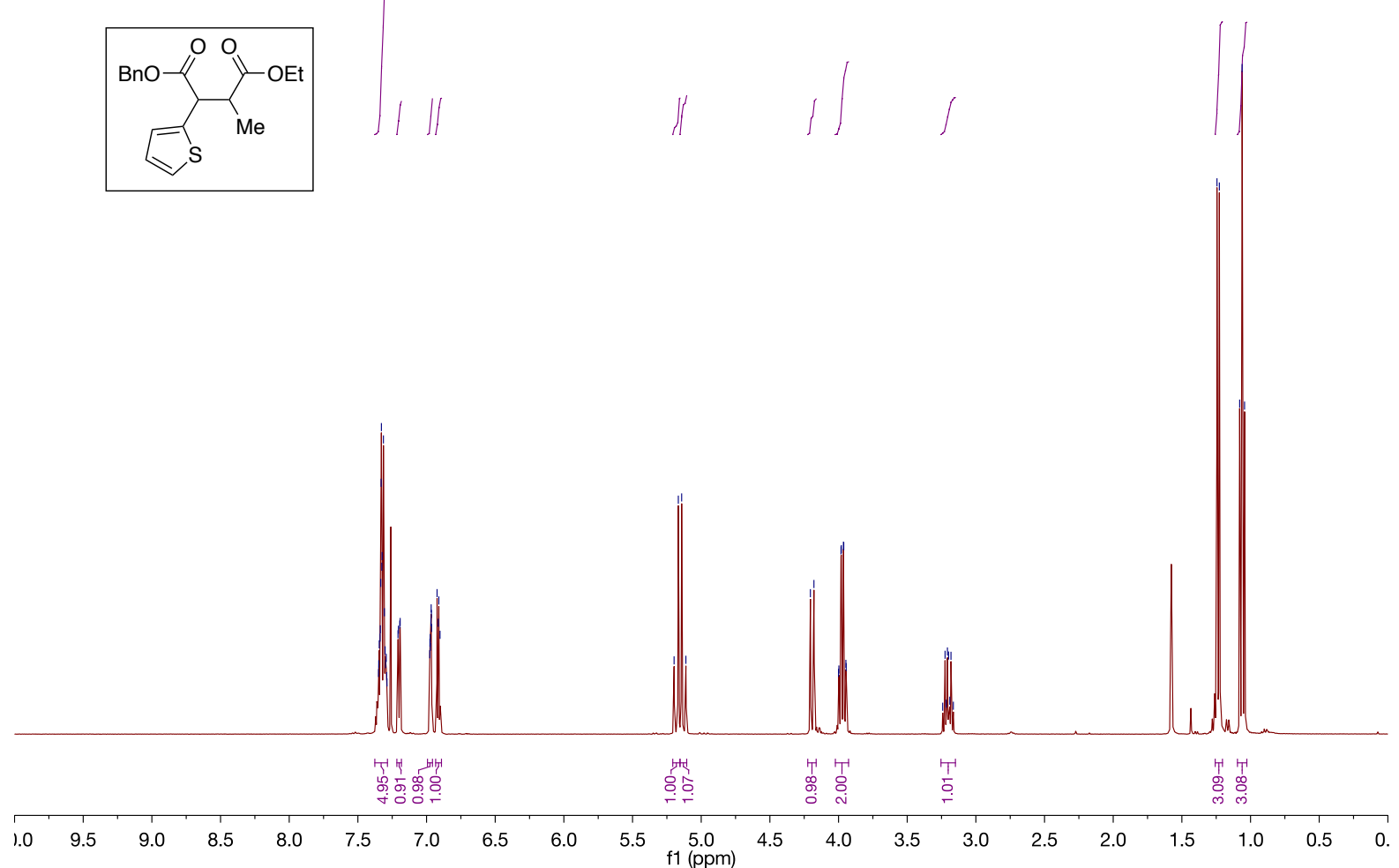




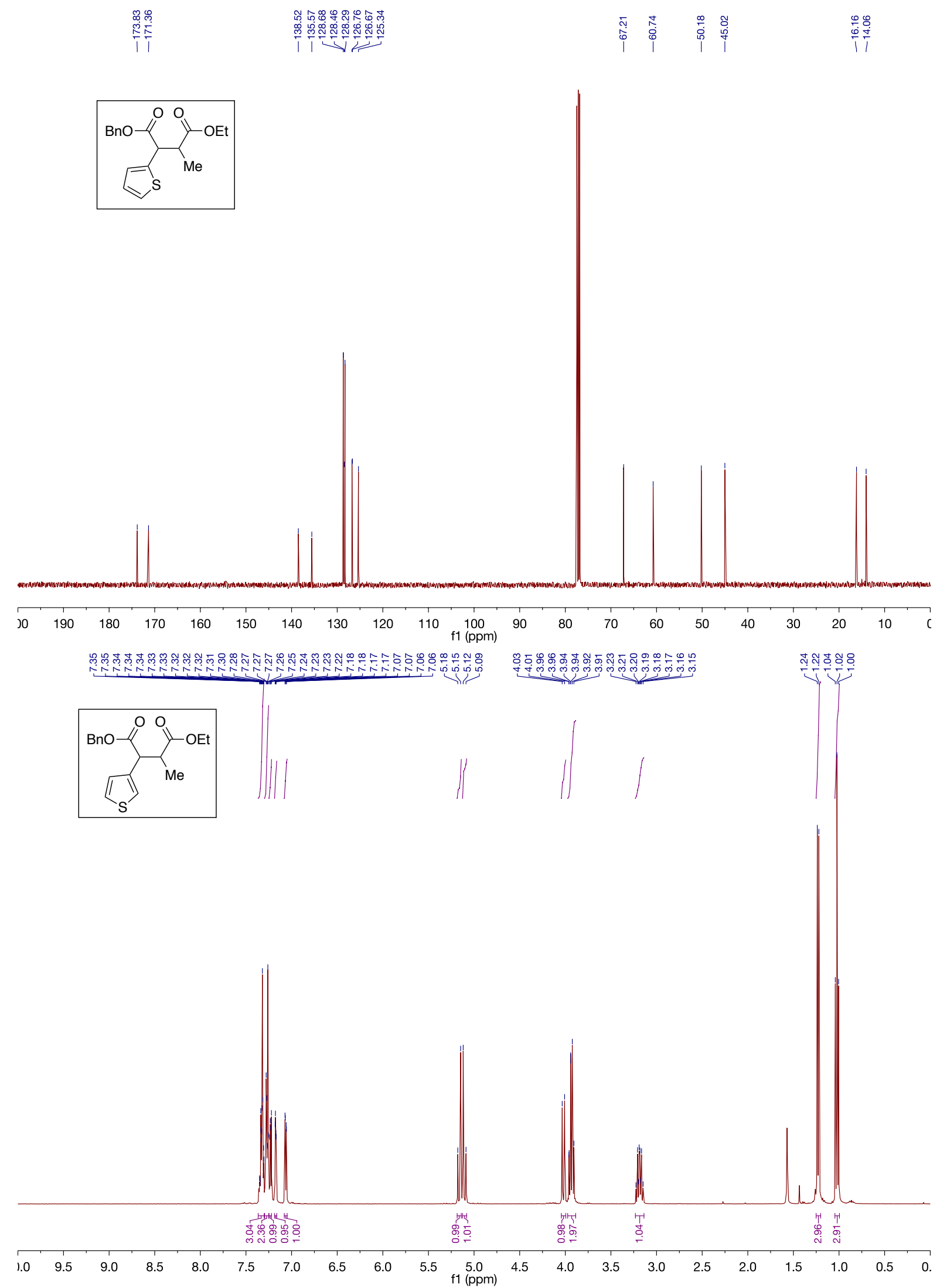



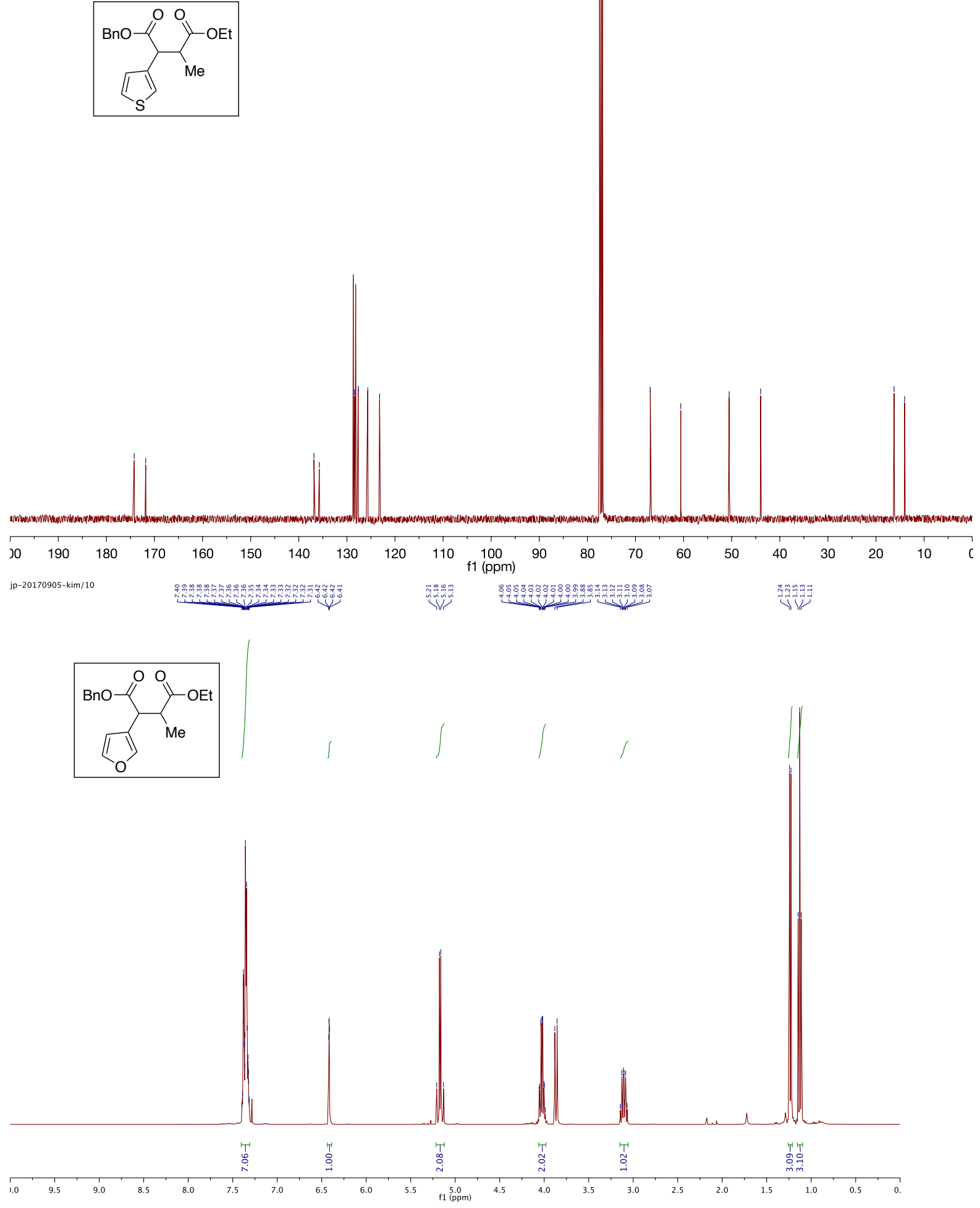

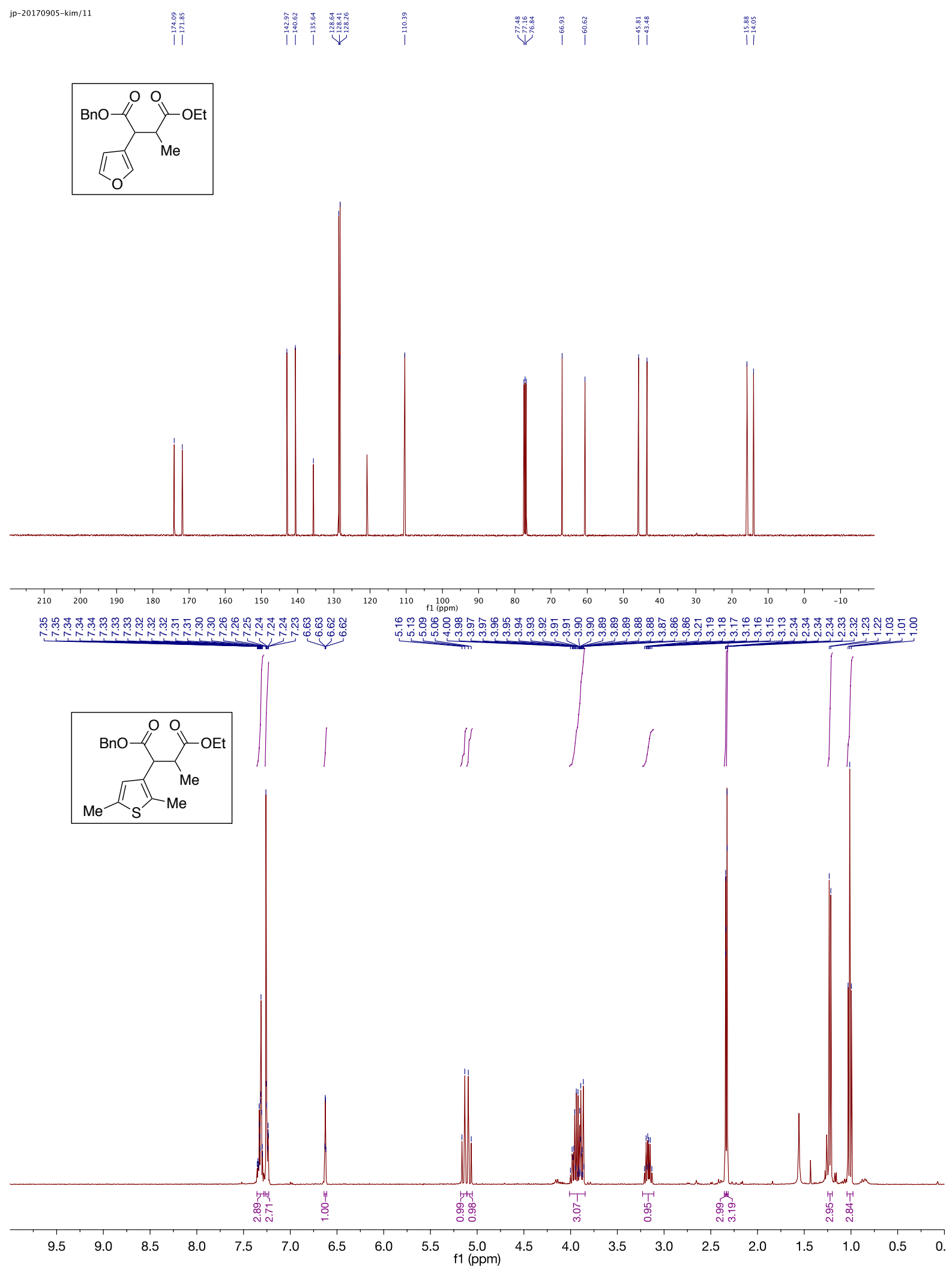

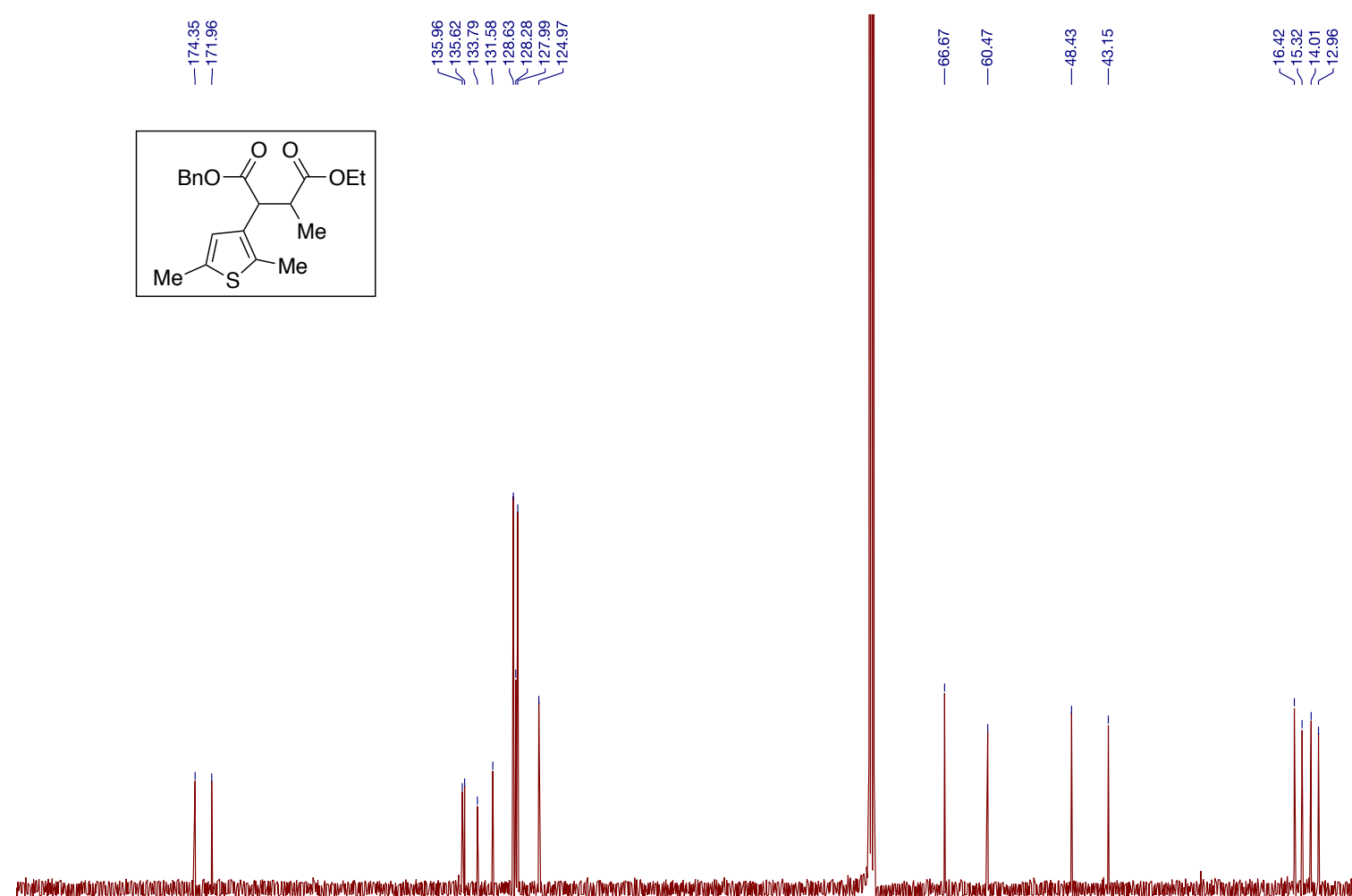

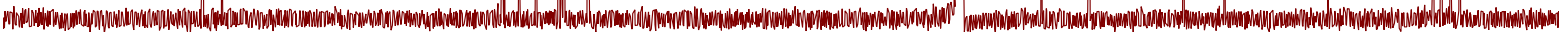

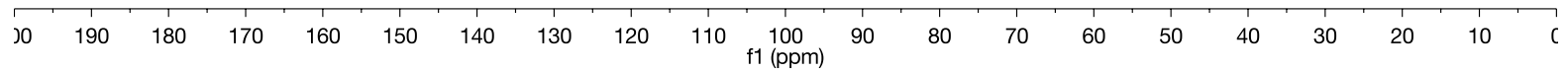

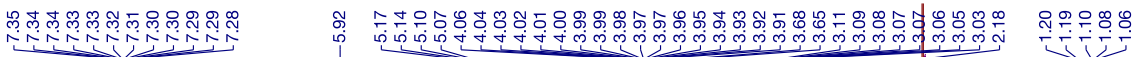
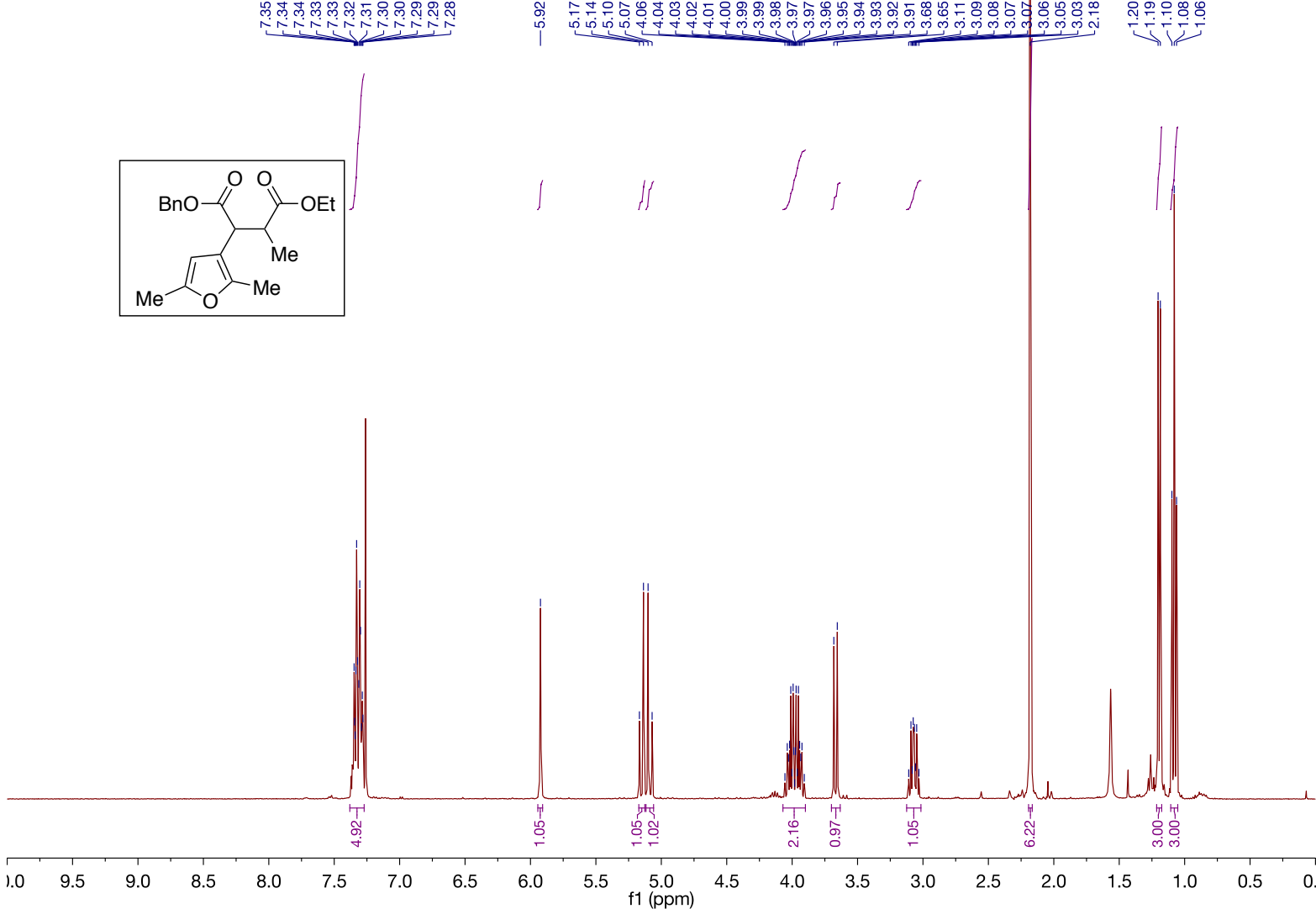

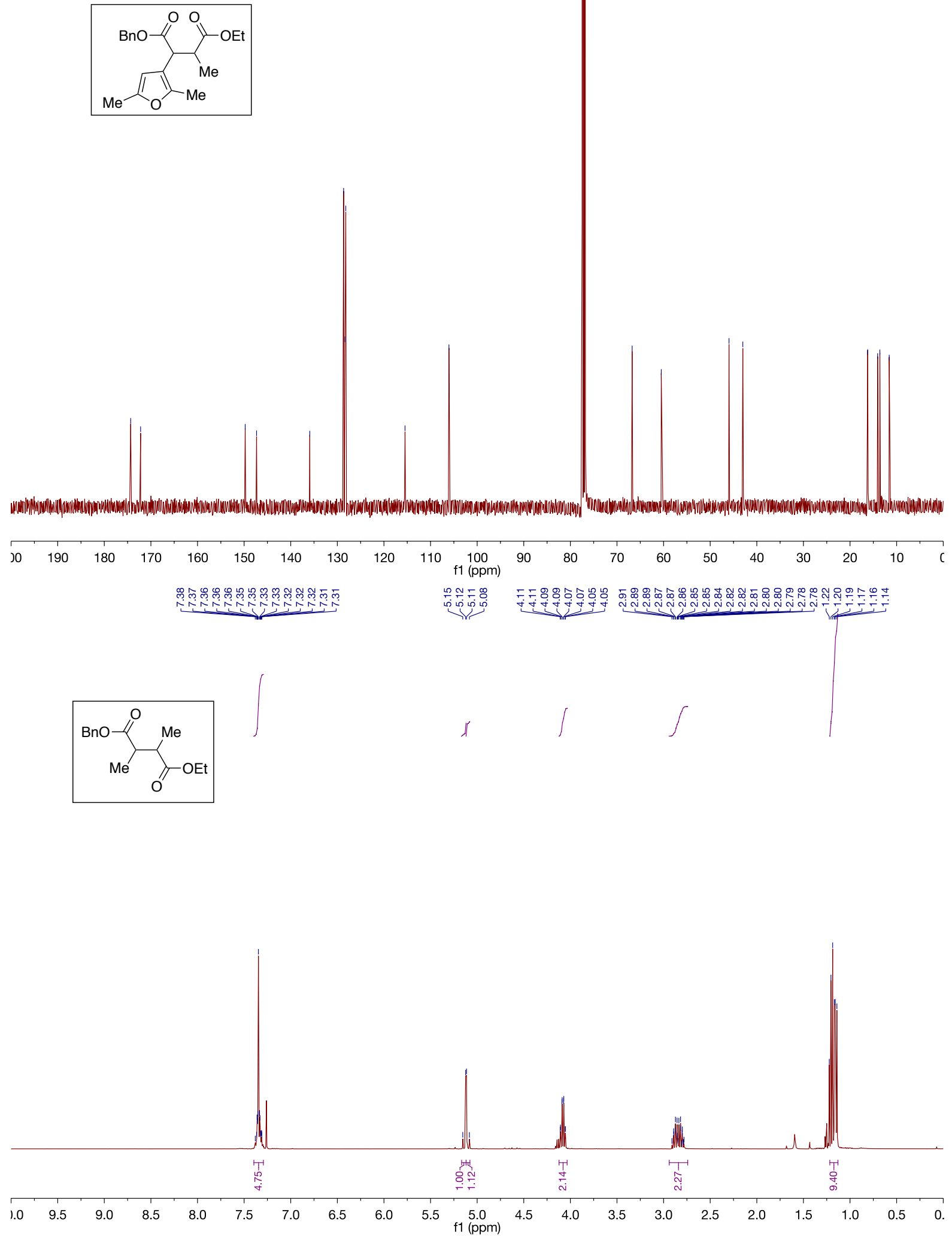


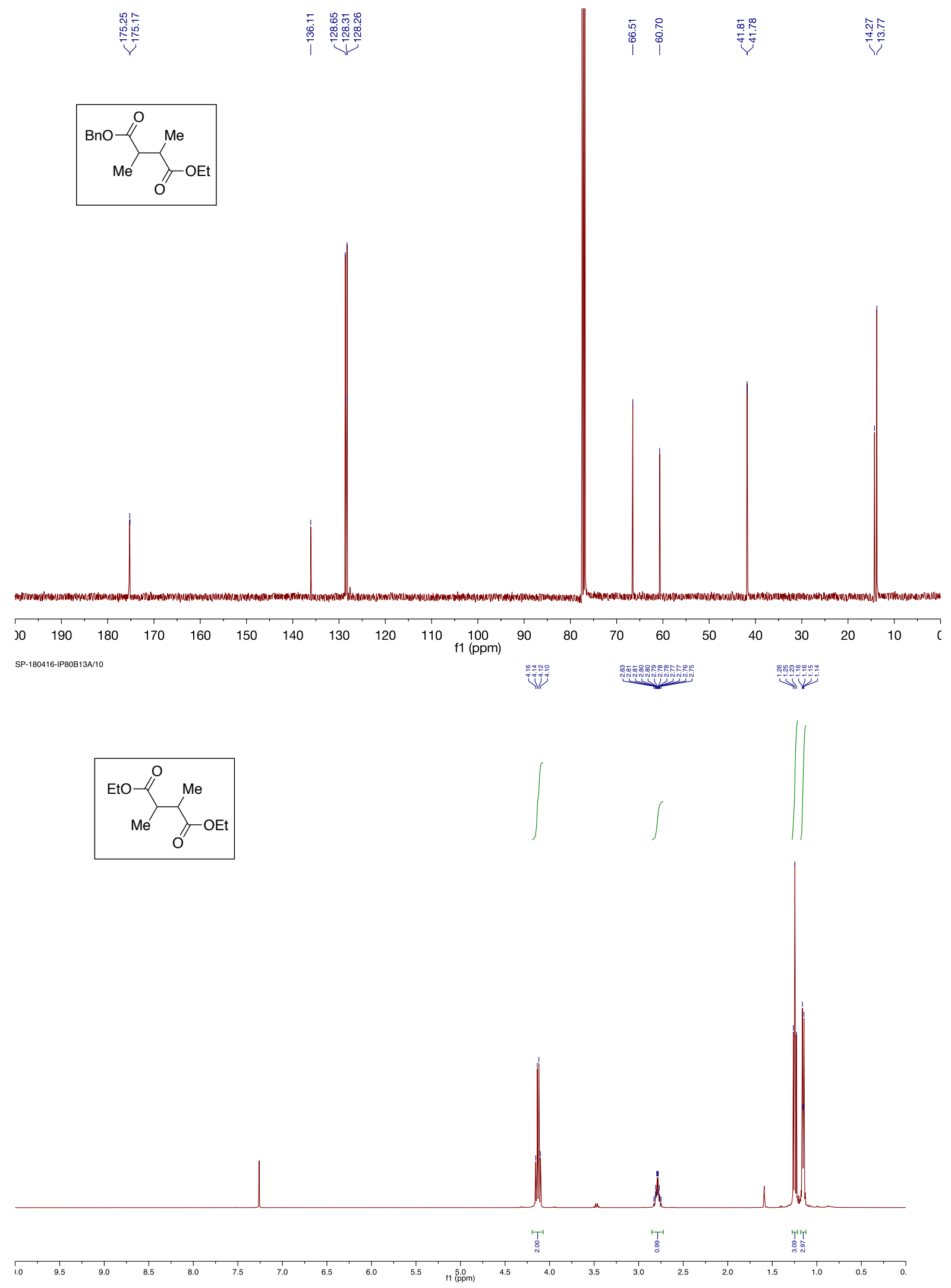

S-139 
SP-180416-IP80B13A/11

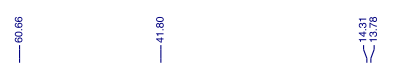

$\sum_{\mathrm{O}}^{\mathrm{MeEt}}$
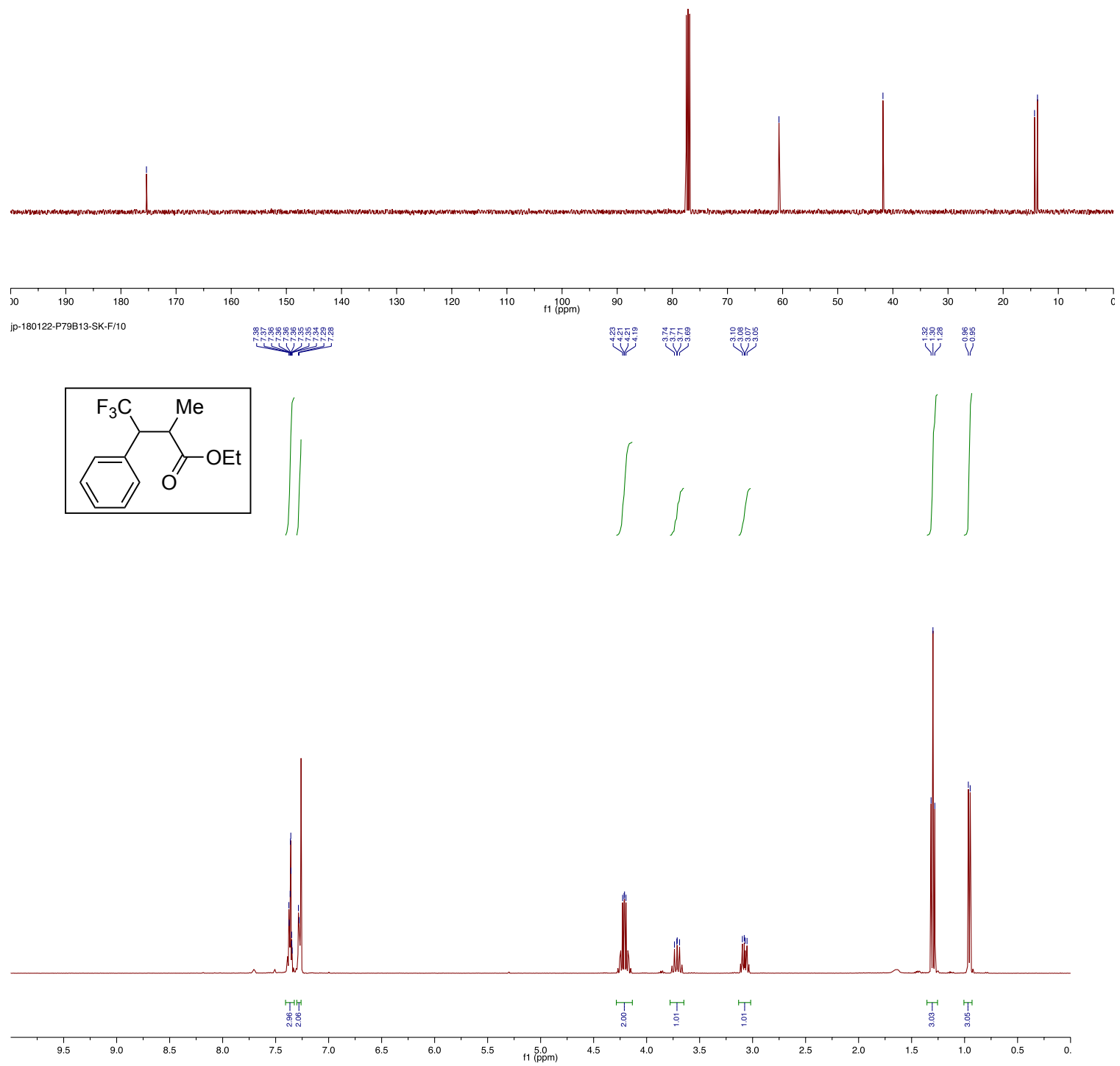

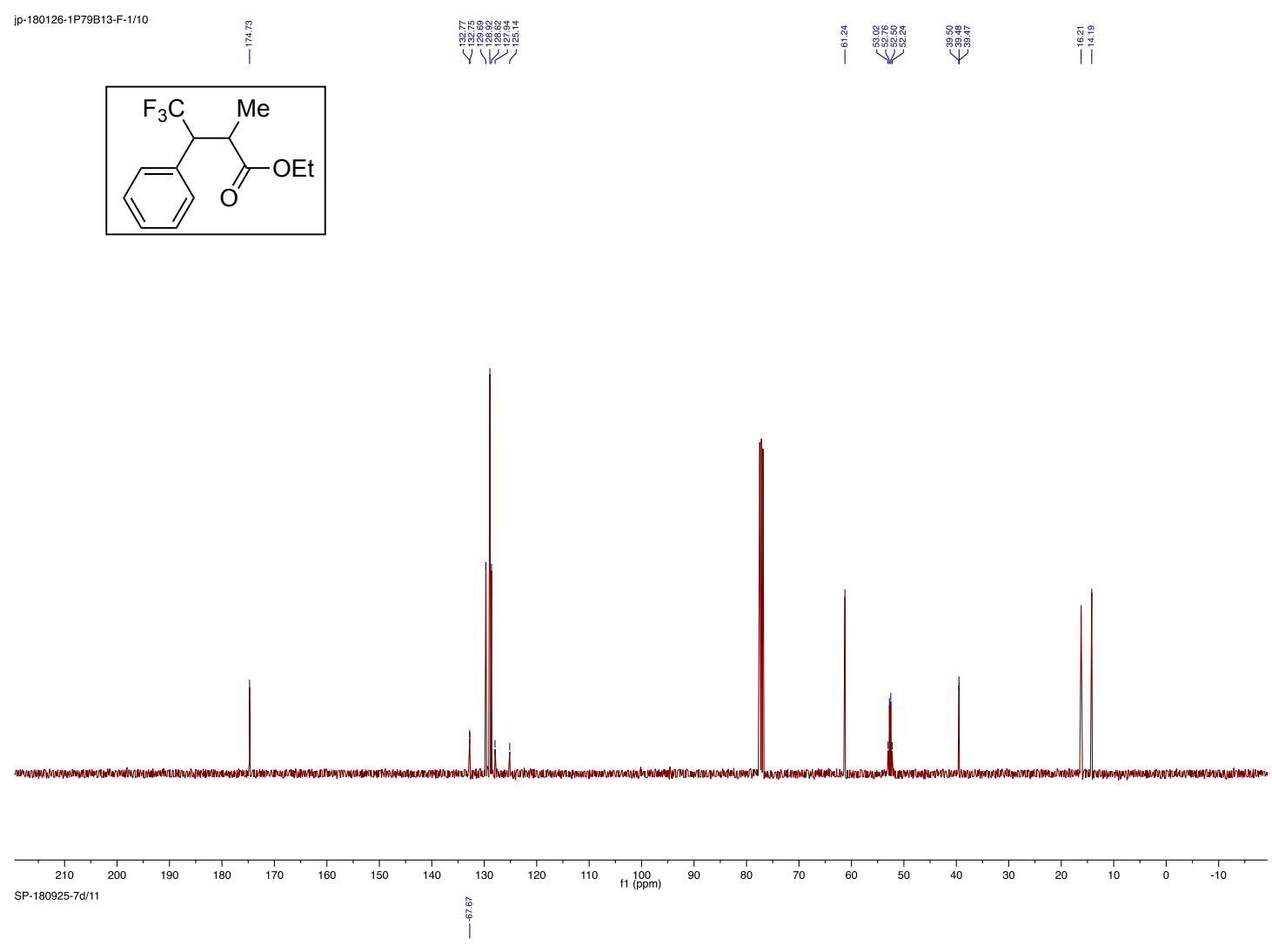

${ }_{\mathrm{O}}^{\mathrm{Me}} \mathrm{OEt}$

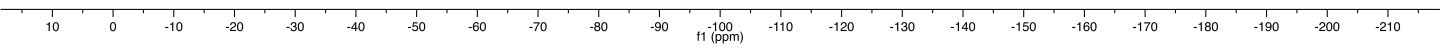




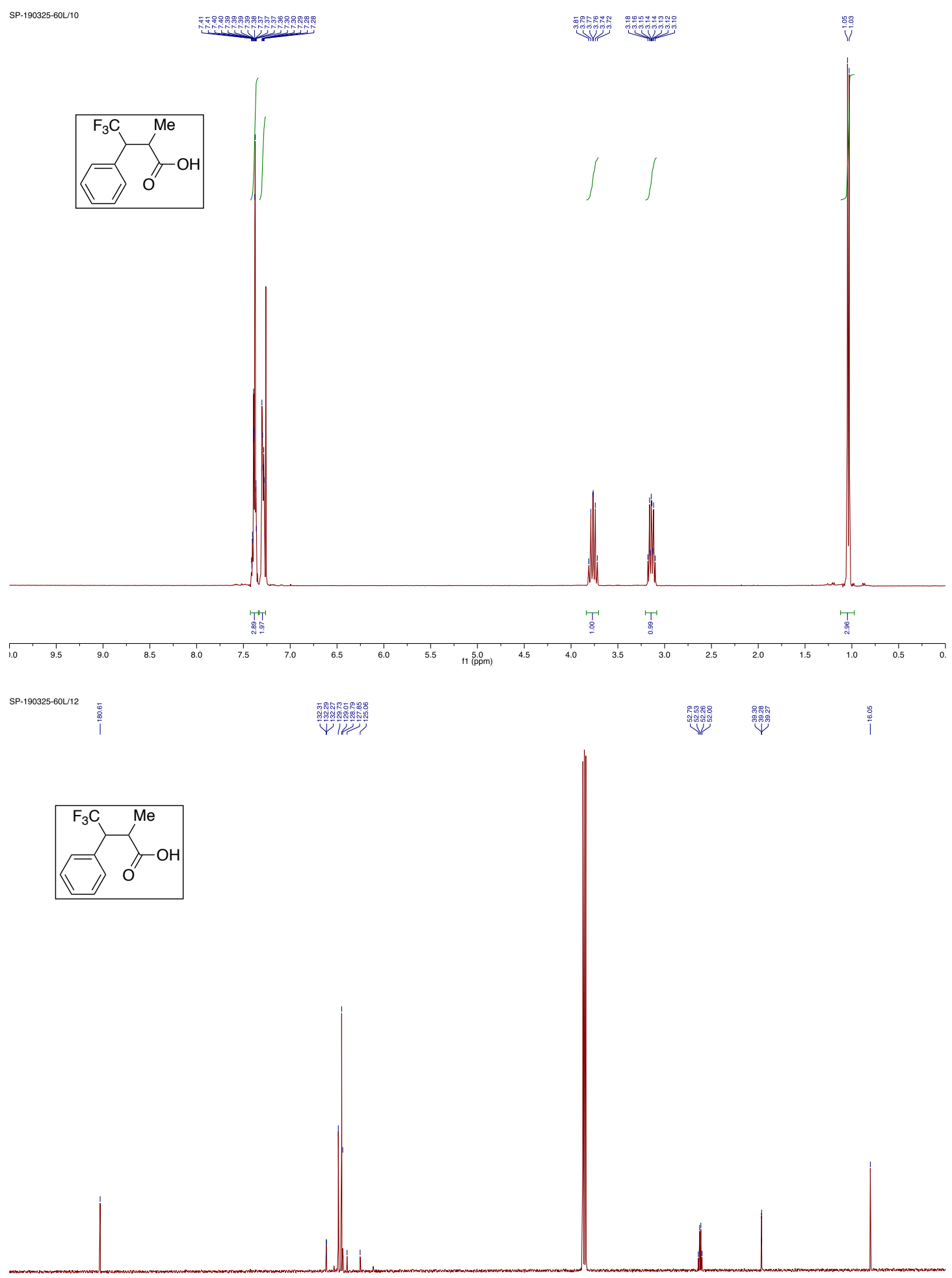

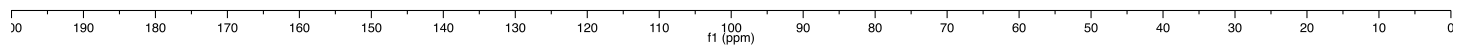



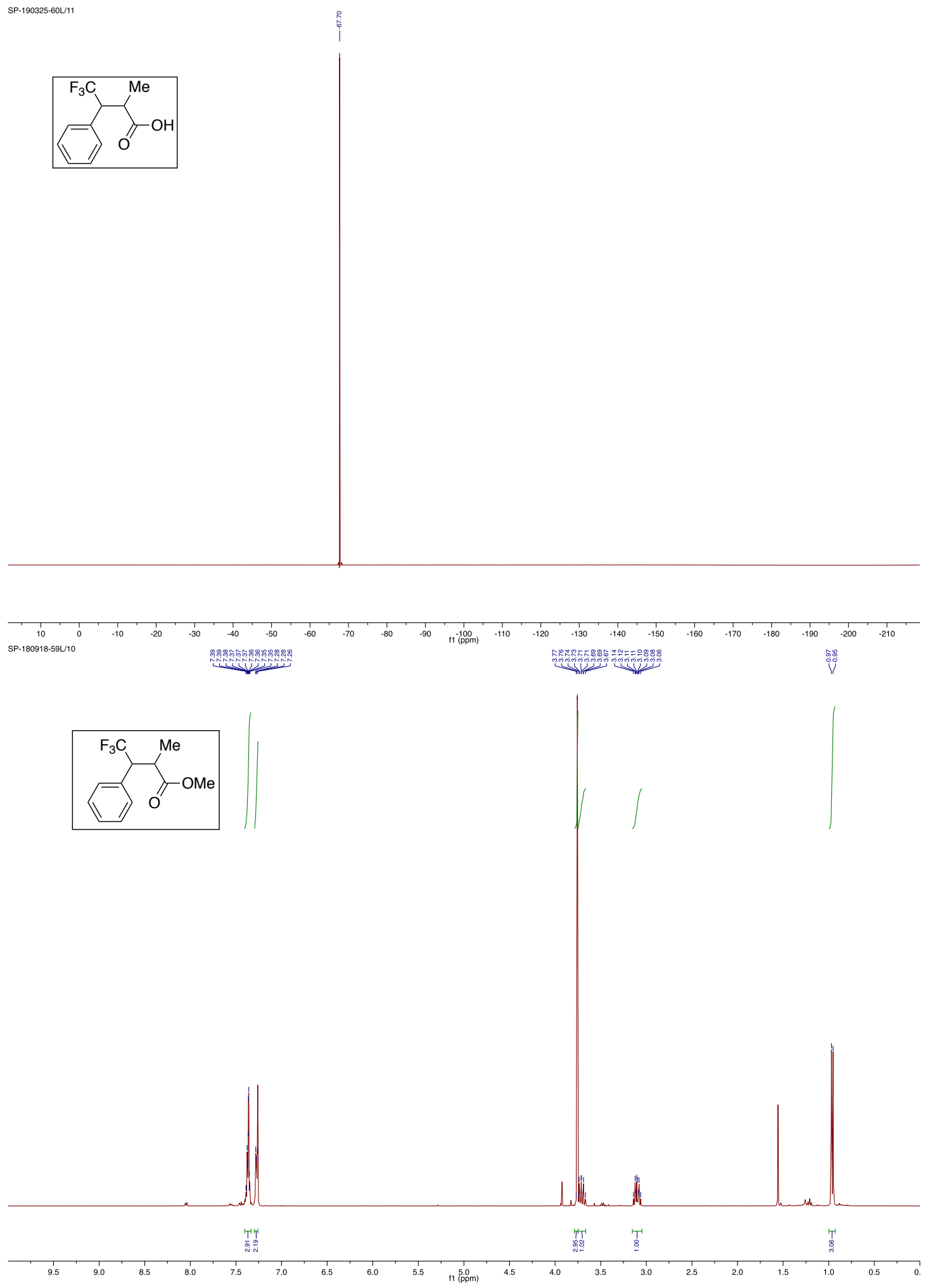

S-143 

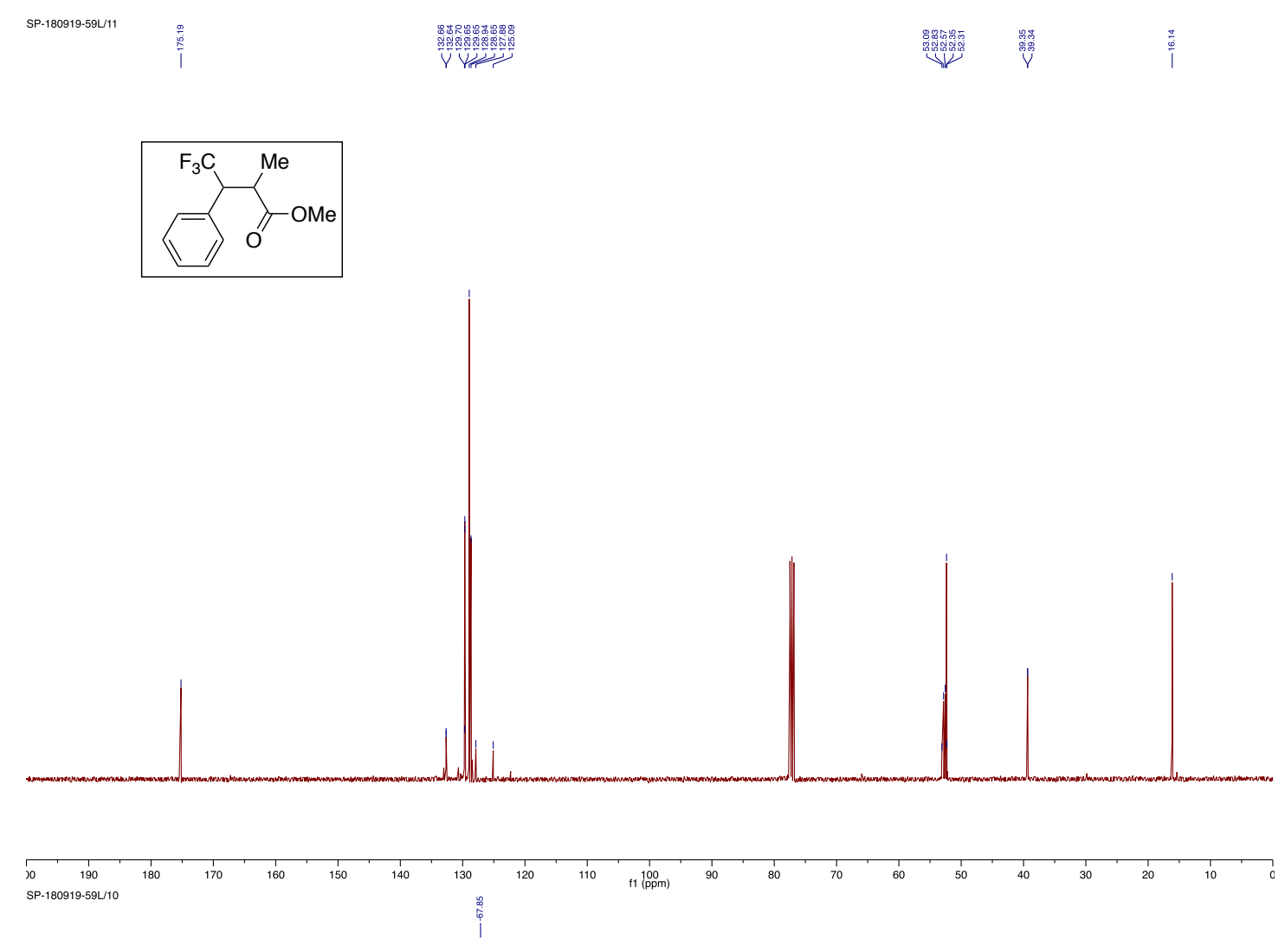

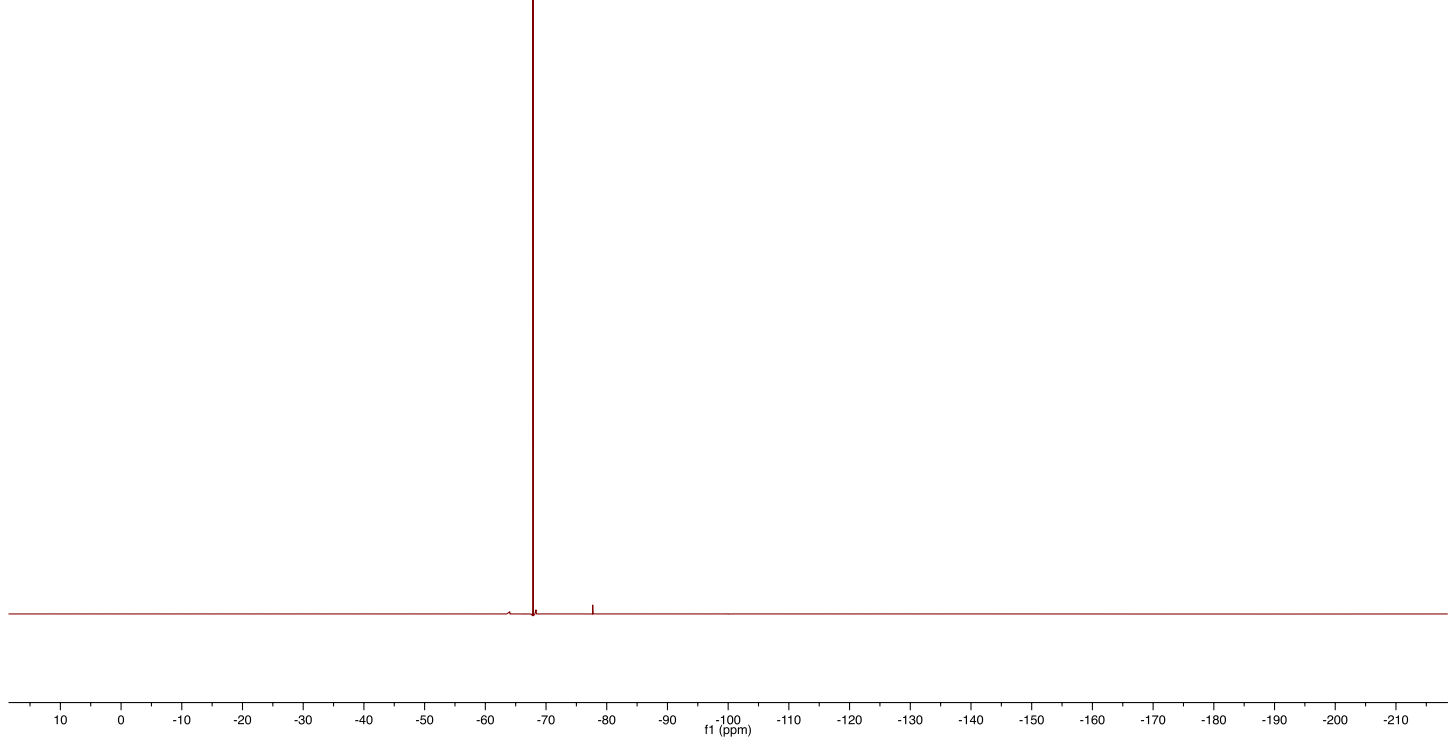
SP-180918-61L10

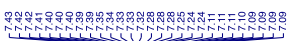
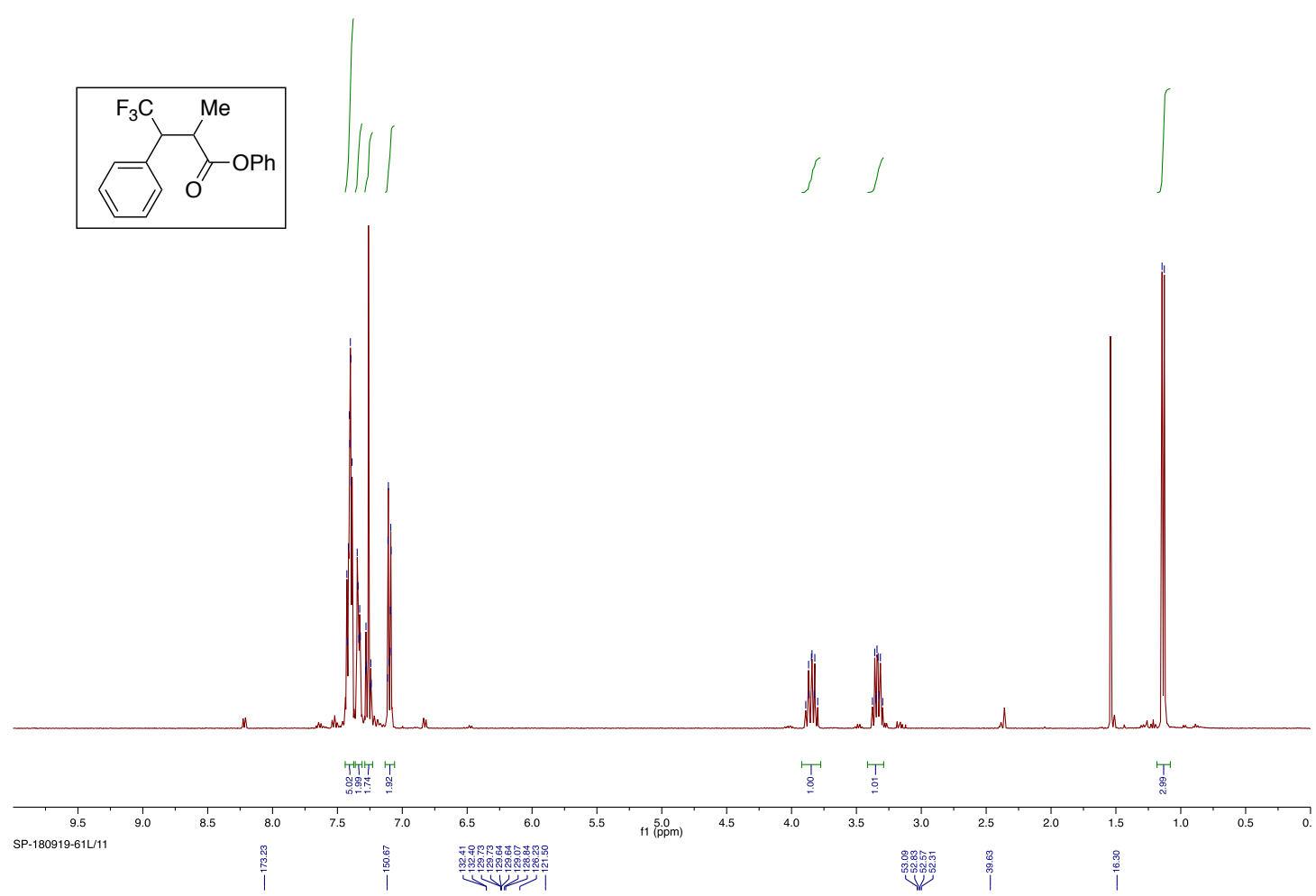

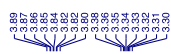
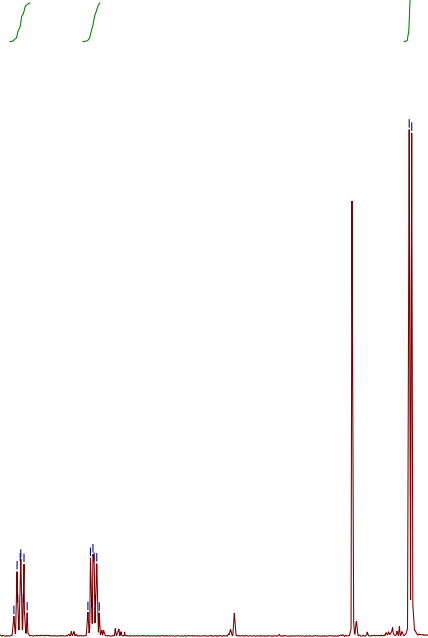
SP-180919-61L10

O
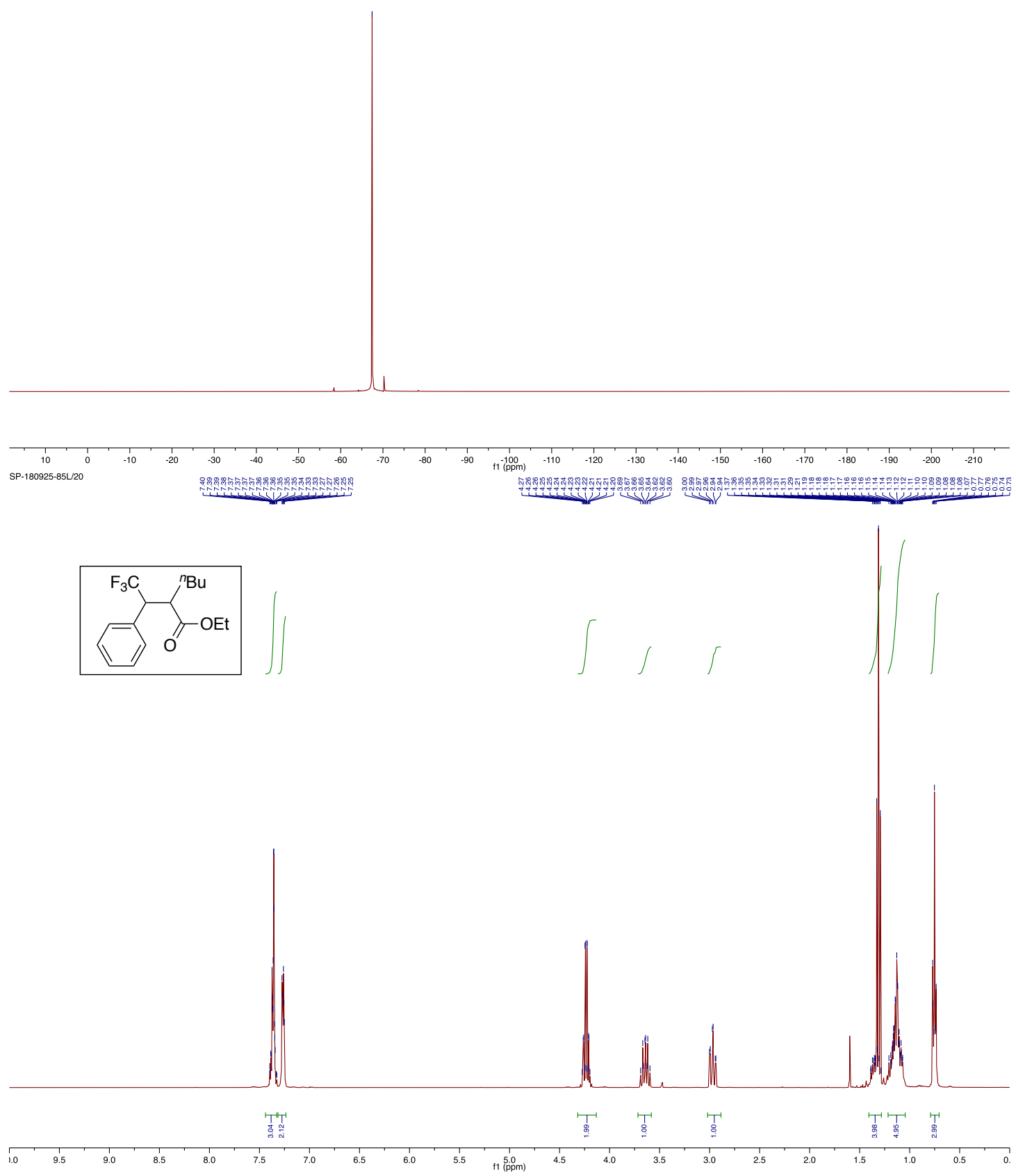

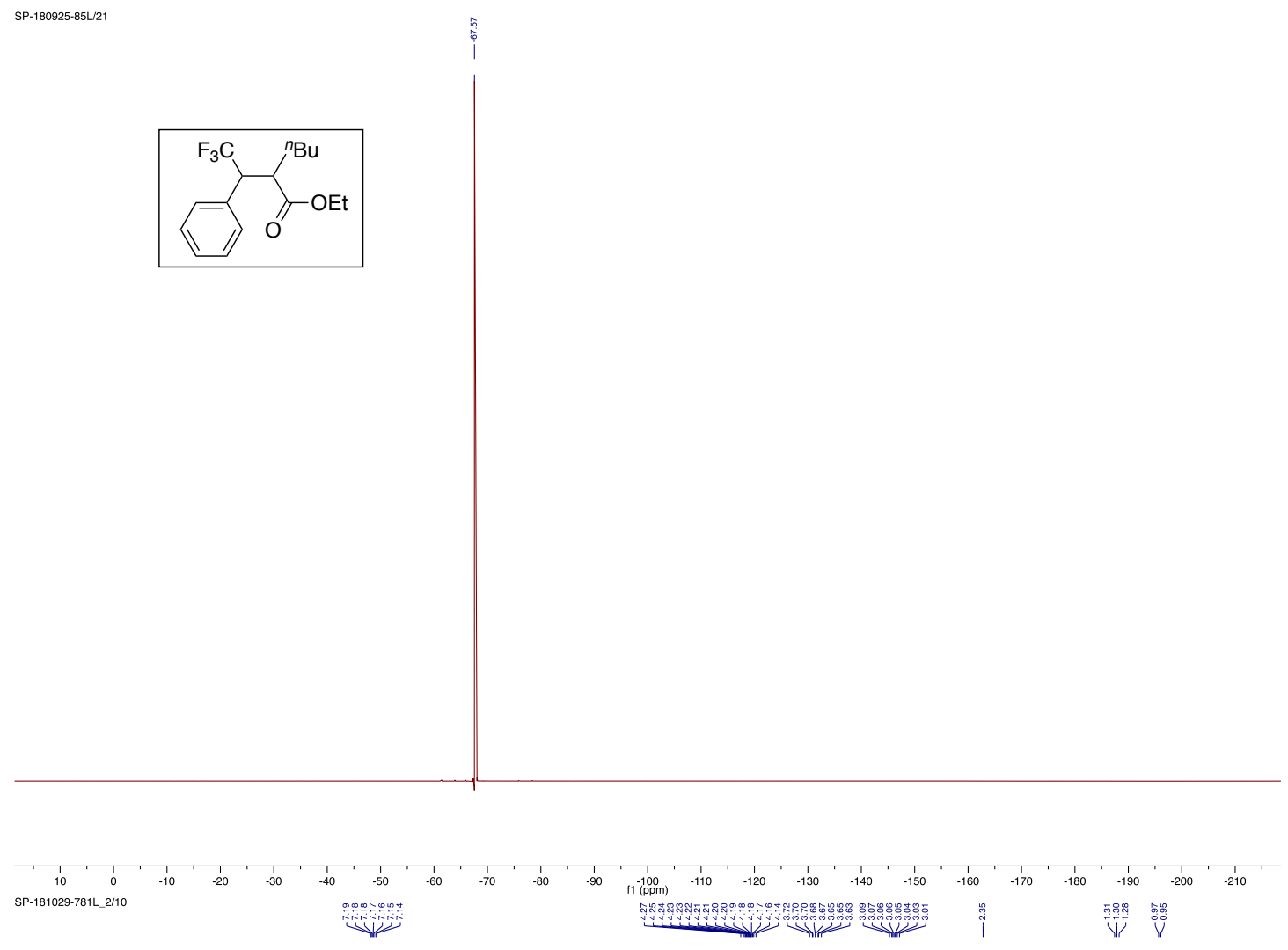
Me
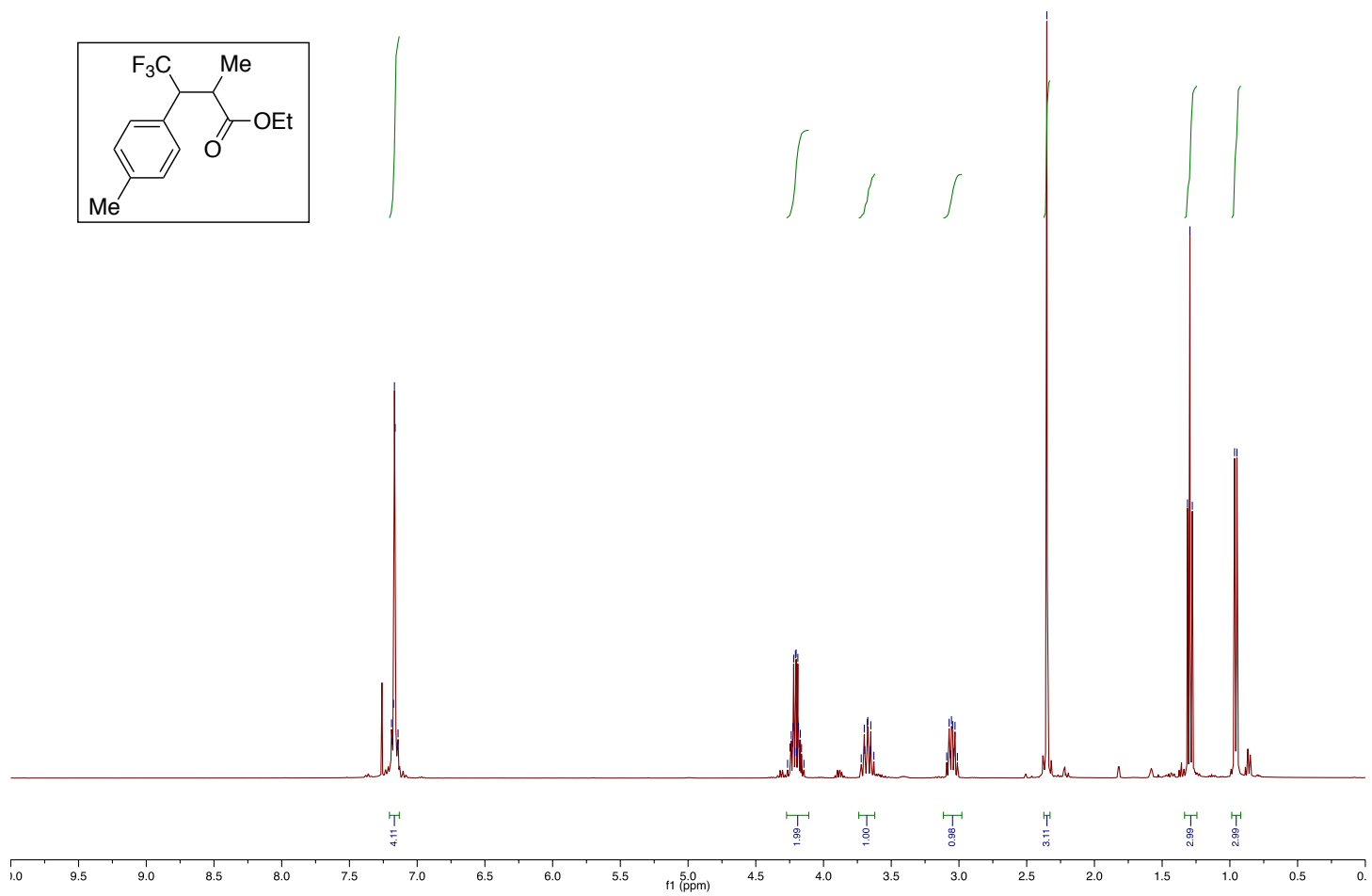

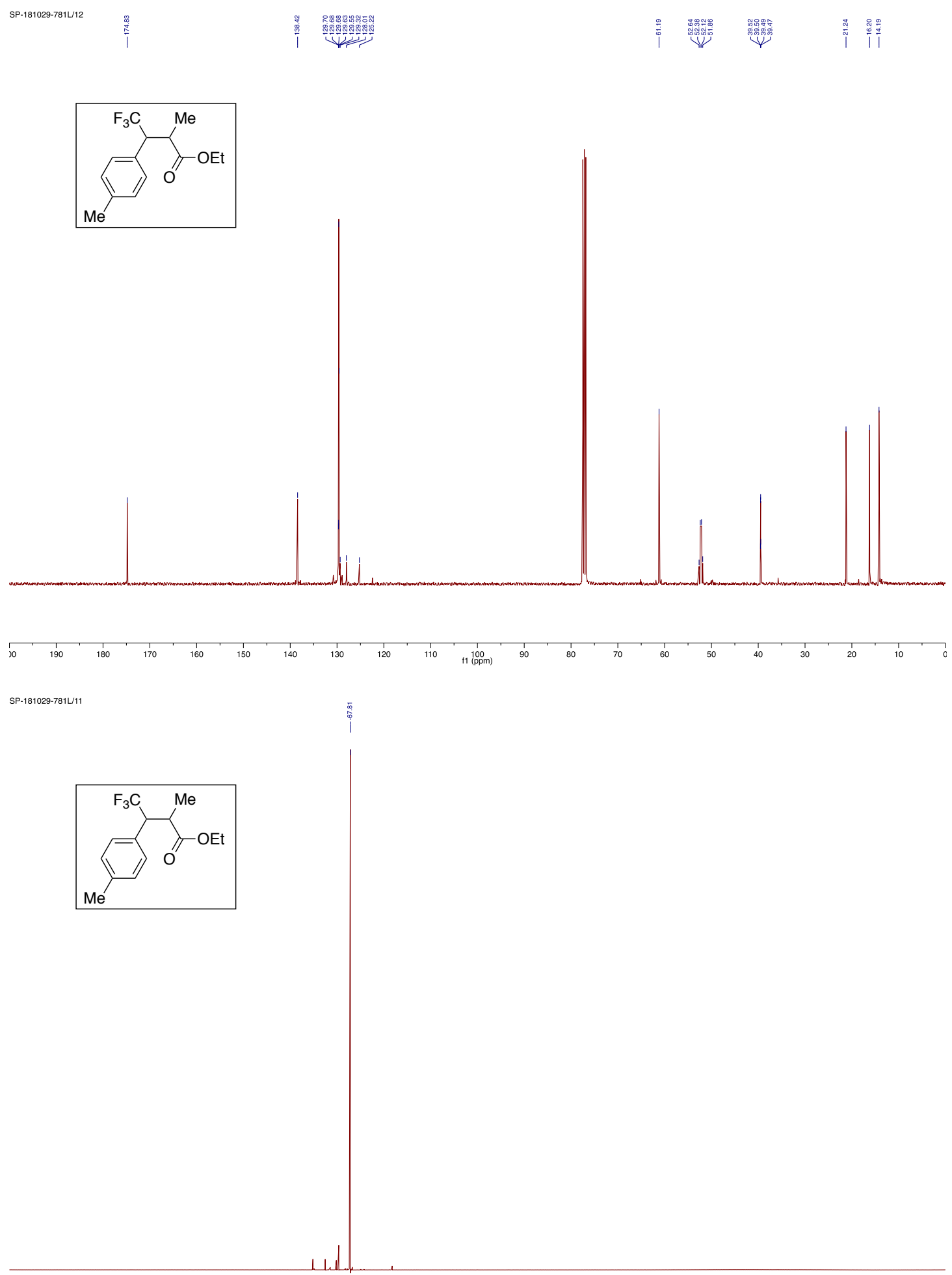

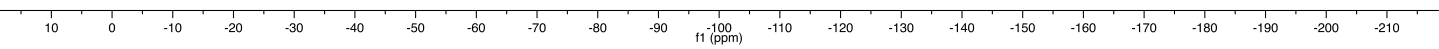


$\overbrace{\mathrm{O}}^{\mathrm{F} C \mathrm{OEt}}$
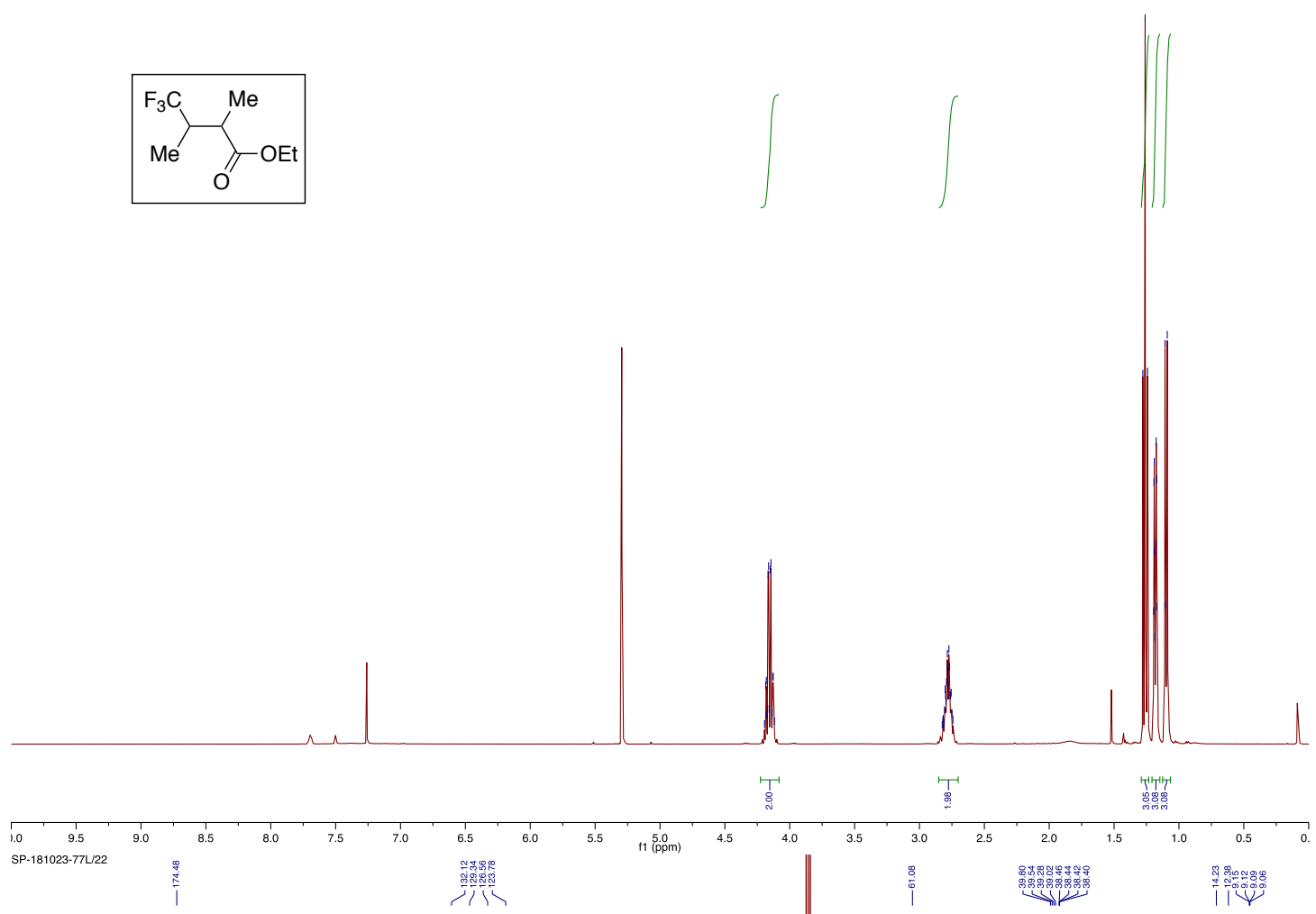

${ }_{M e}^{F_{3} C} \prod_{O}^{M e}$

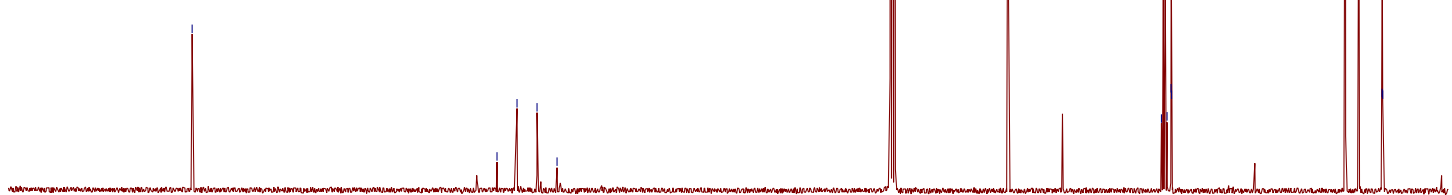

S-149 

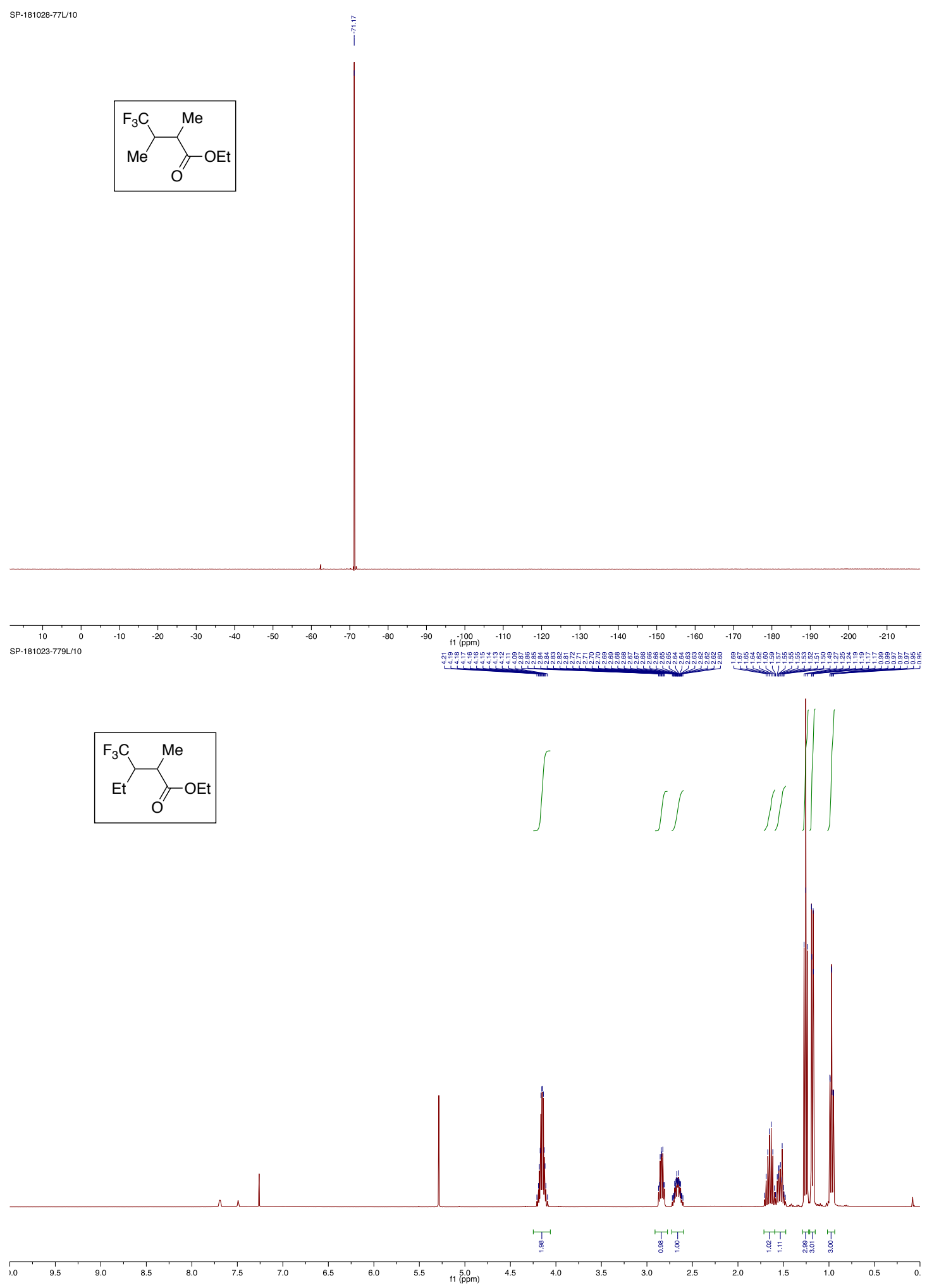

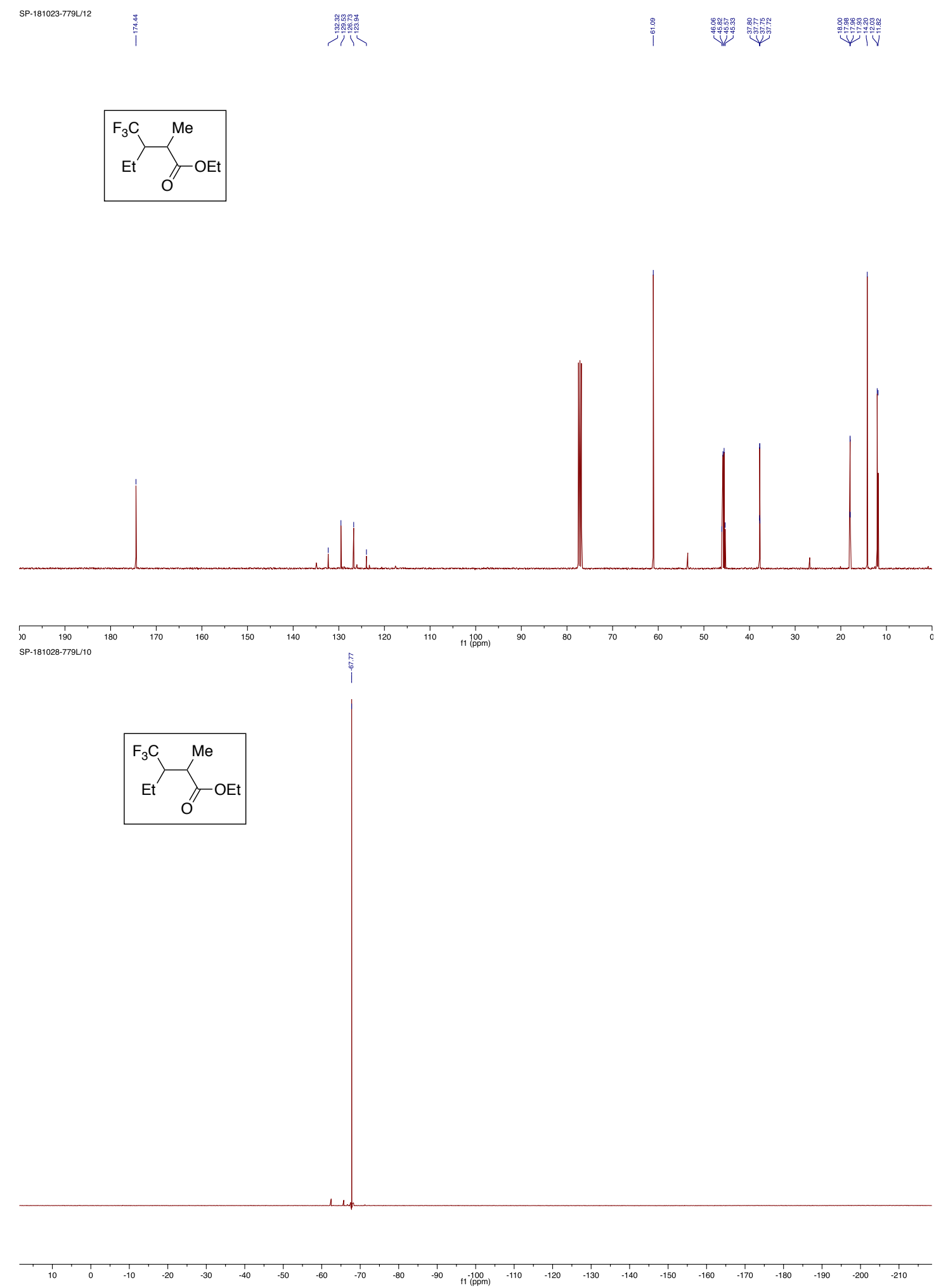

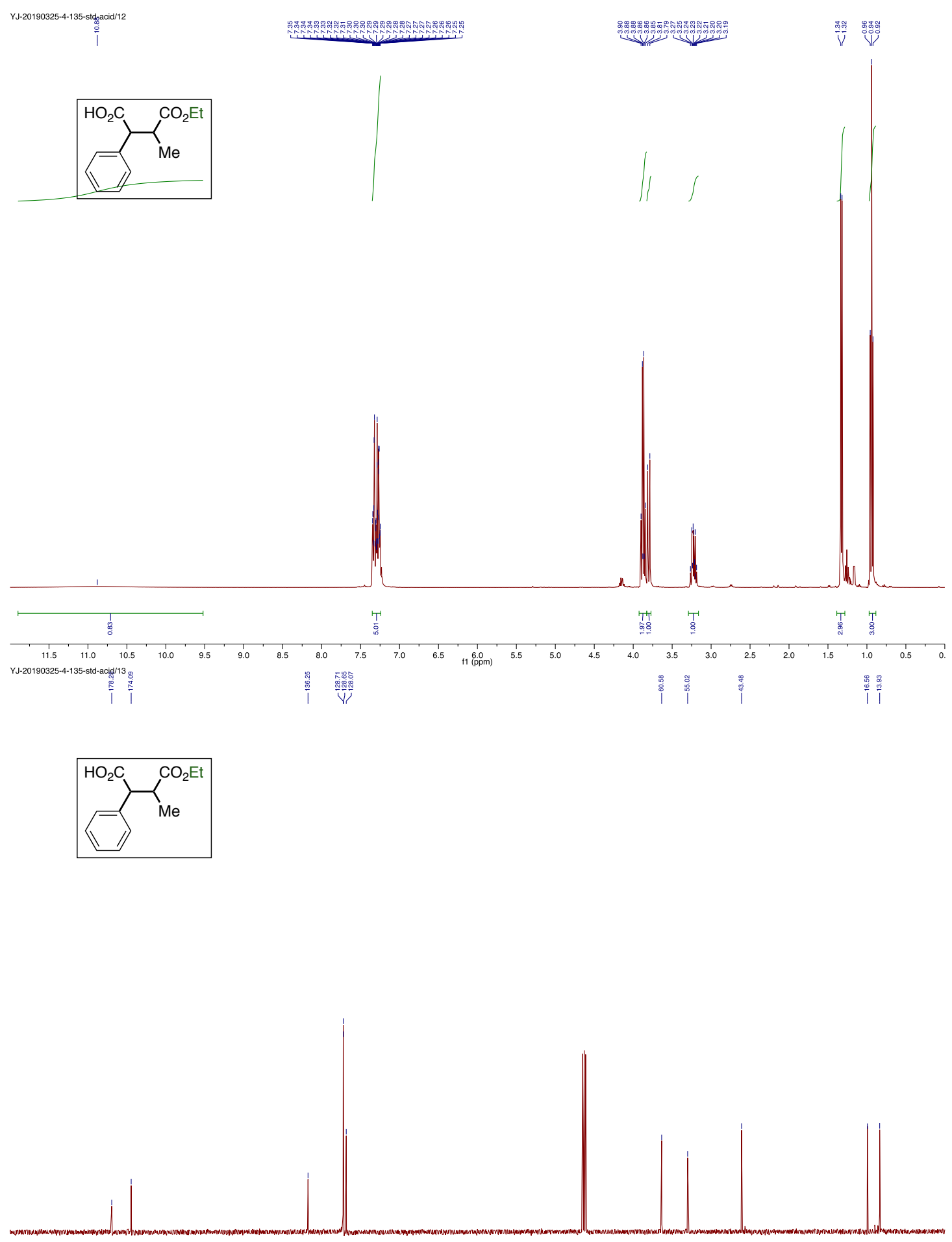

S-152 
ip-190118-4-44-2/10
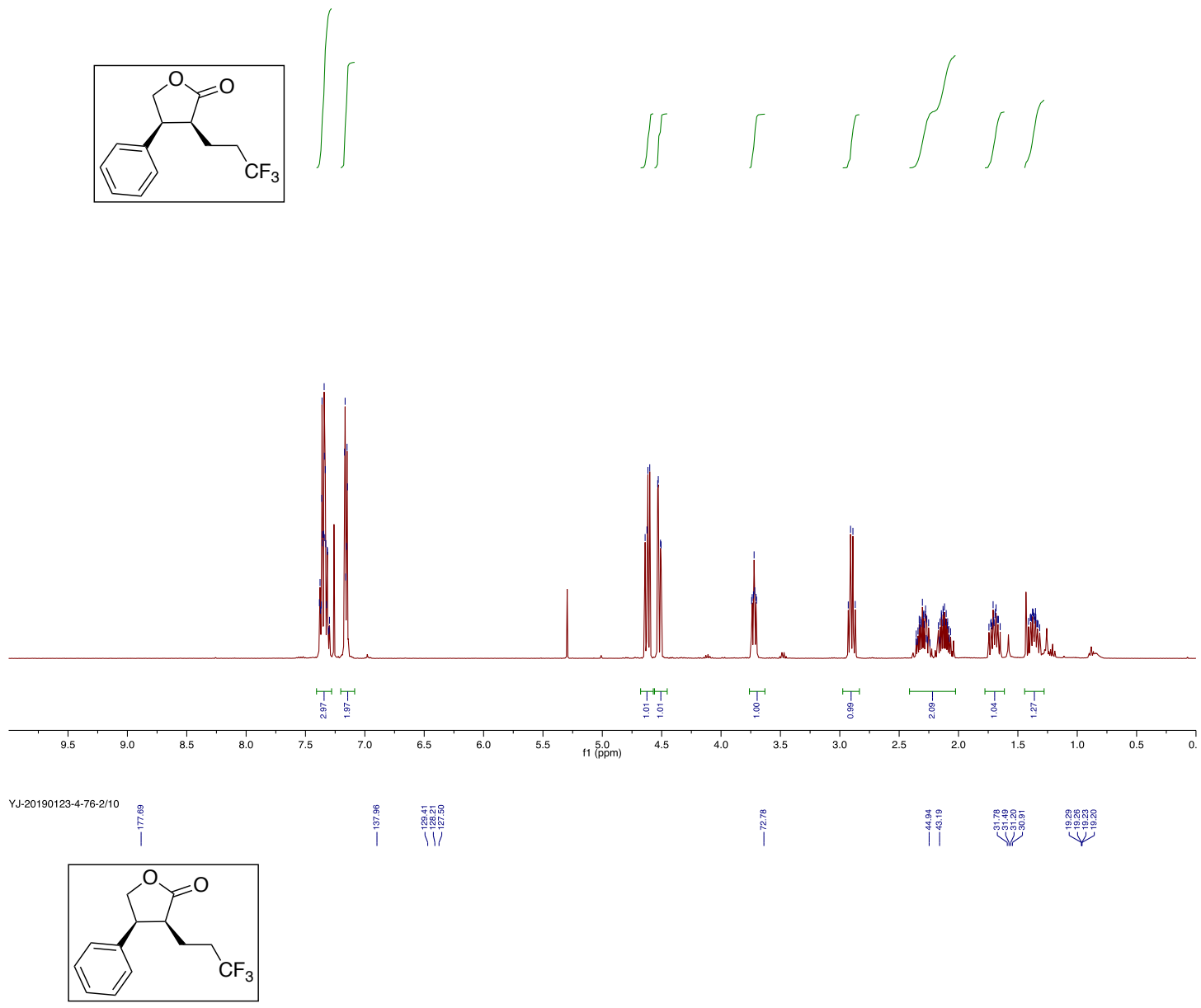

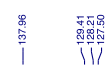

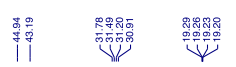

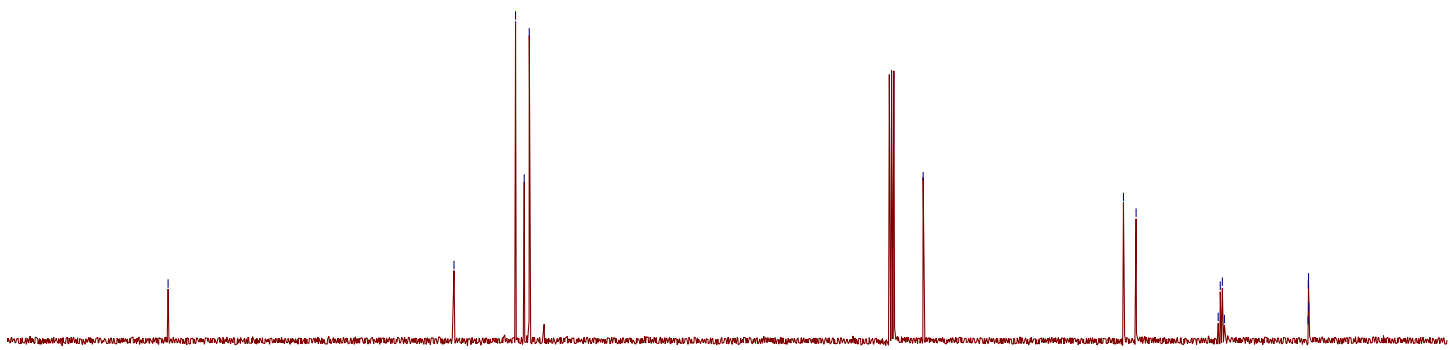

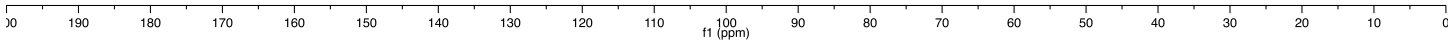



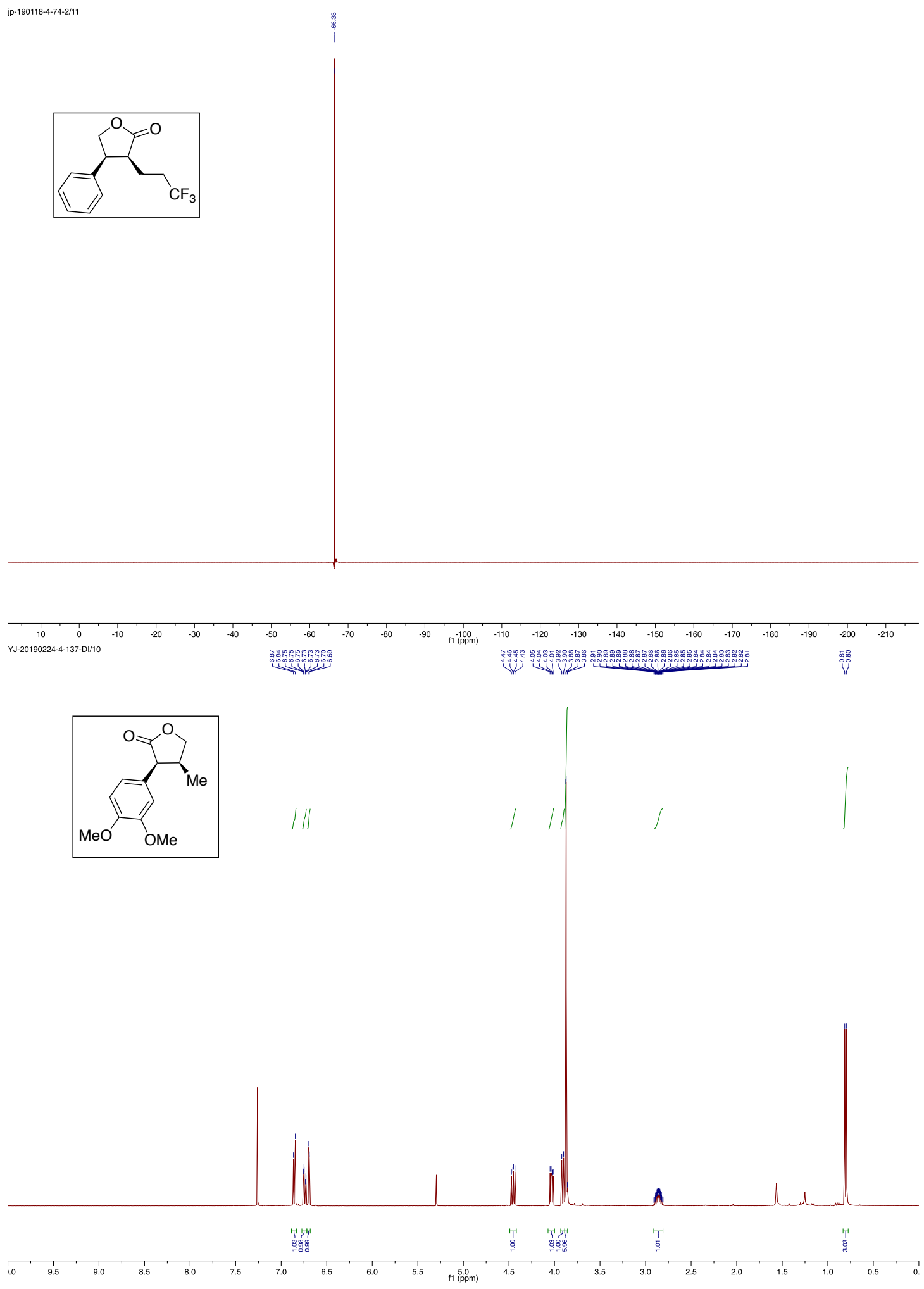

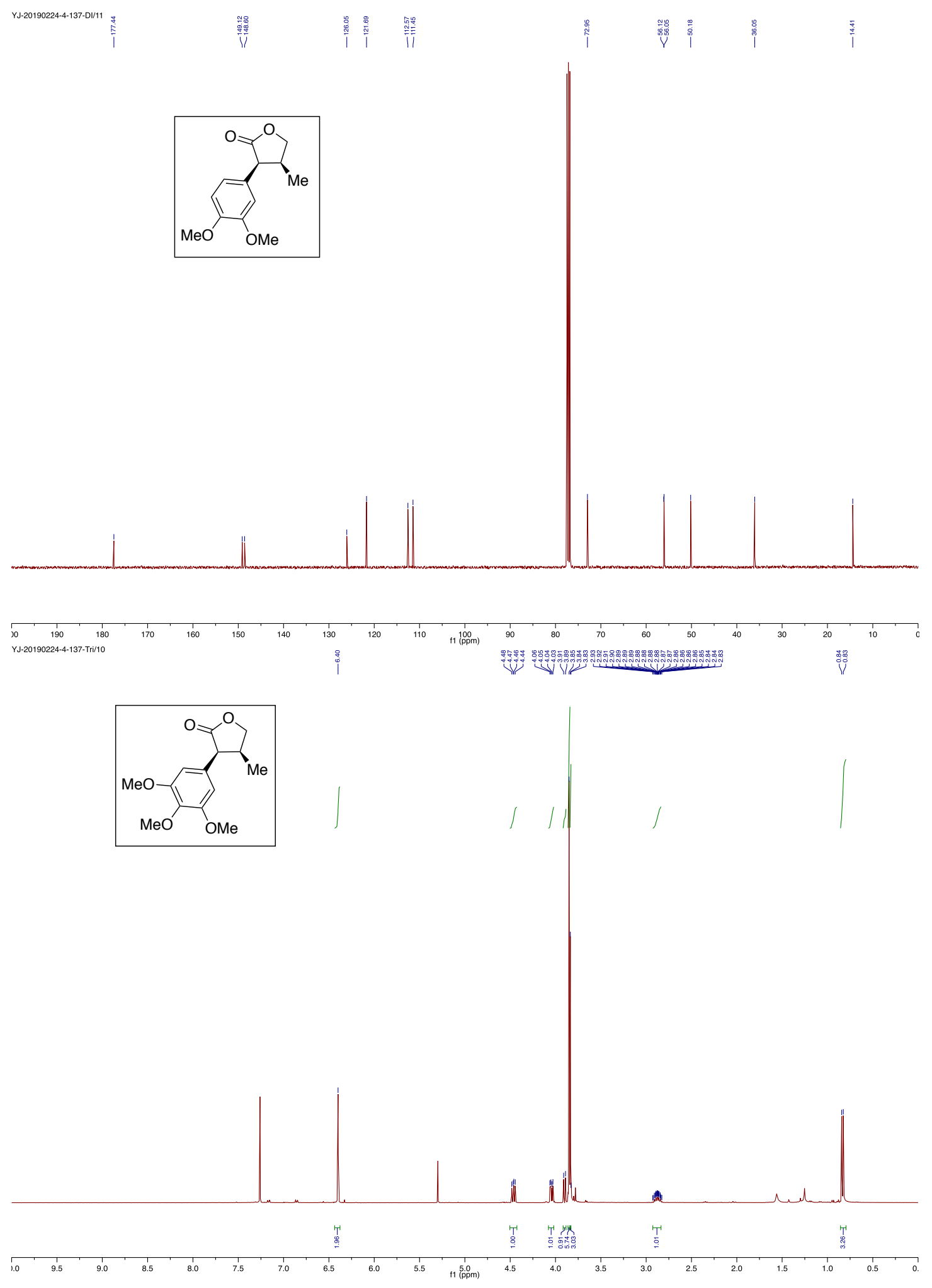

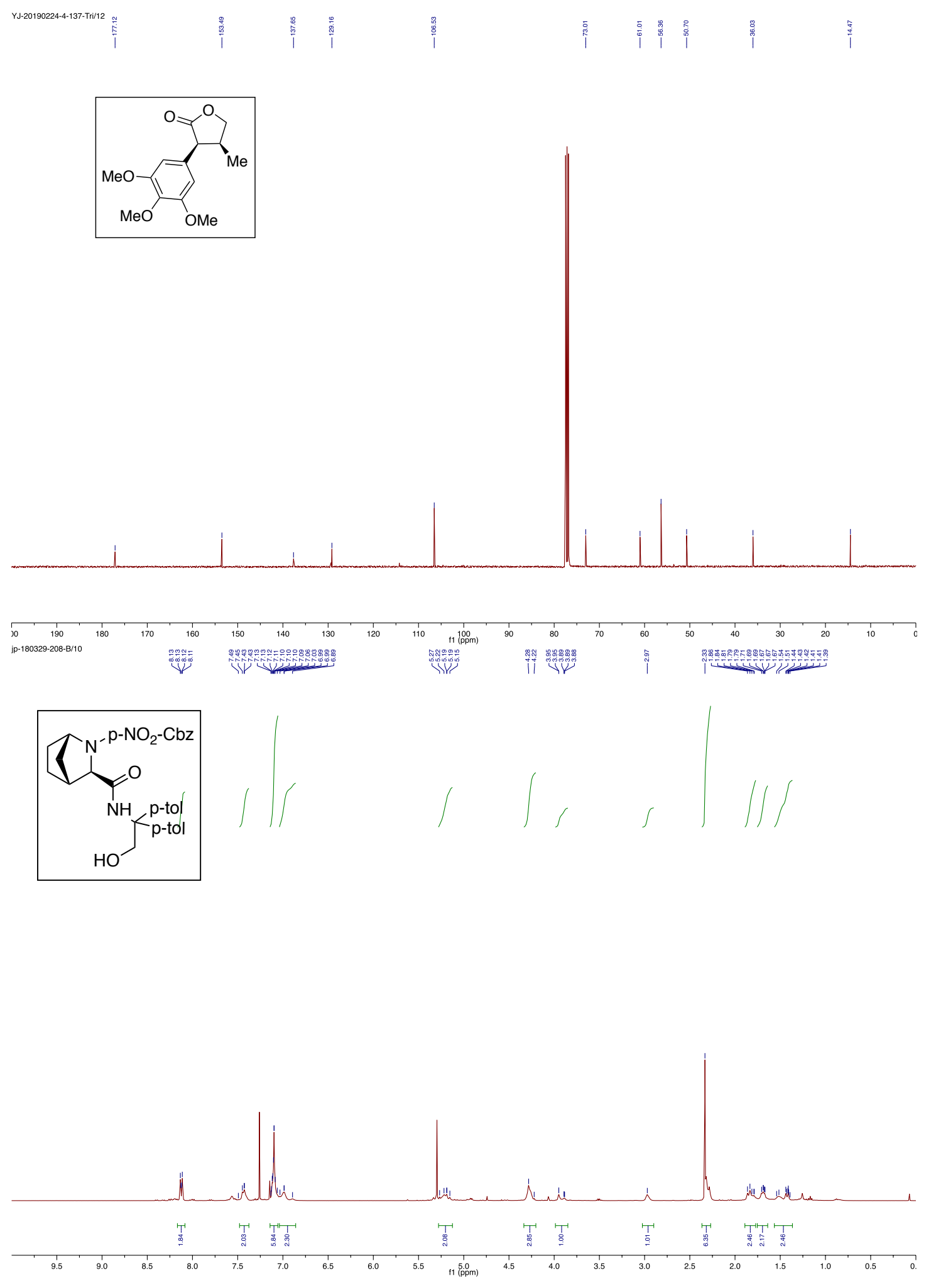

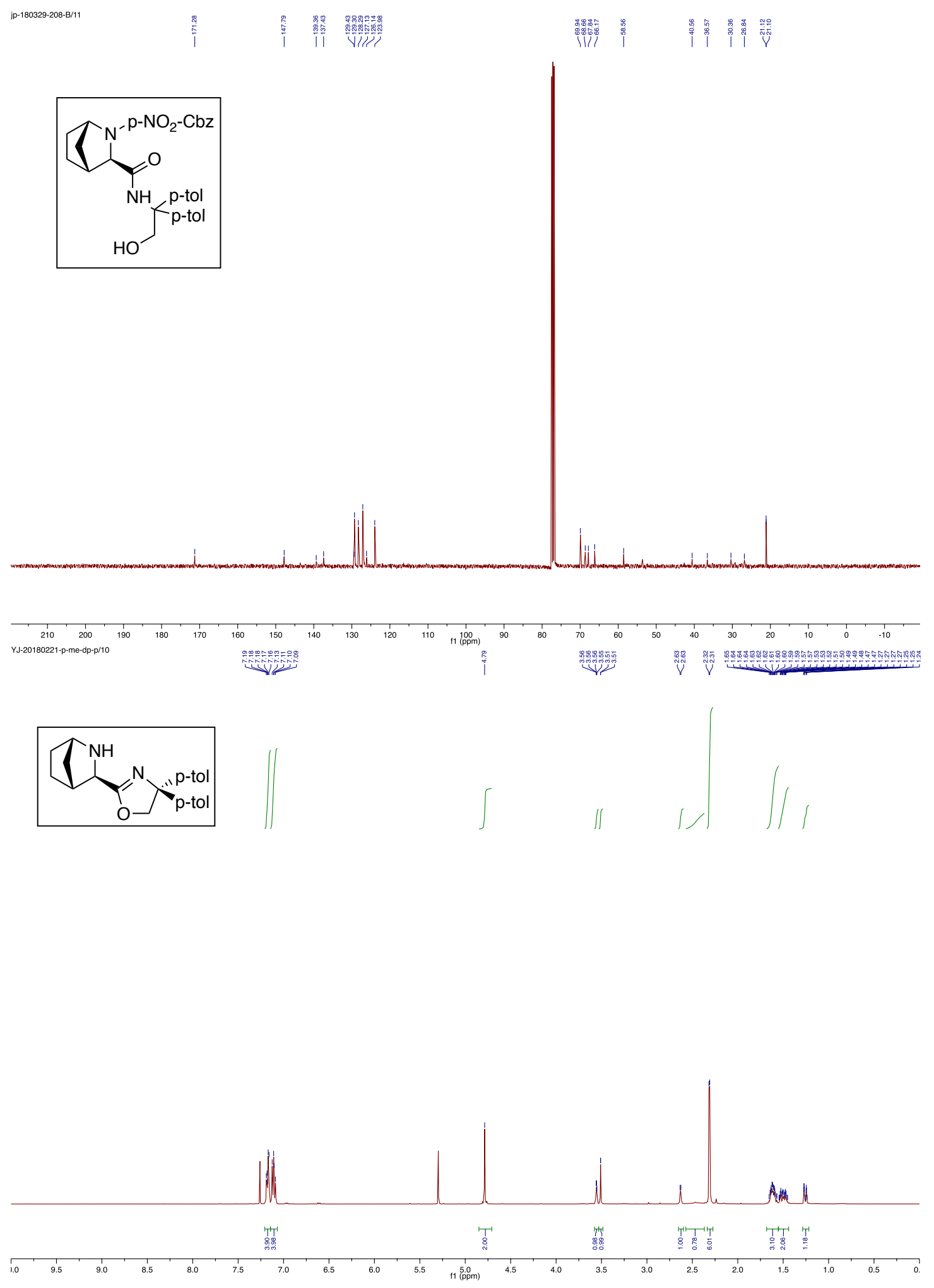
YJ-20180221-p-me-dp-p/11

$$
\overbrace{0}^{N H} N_{p-t o l}^{N}
$$
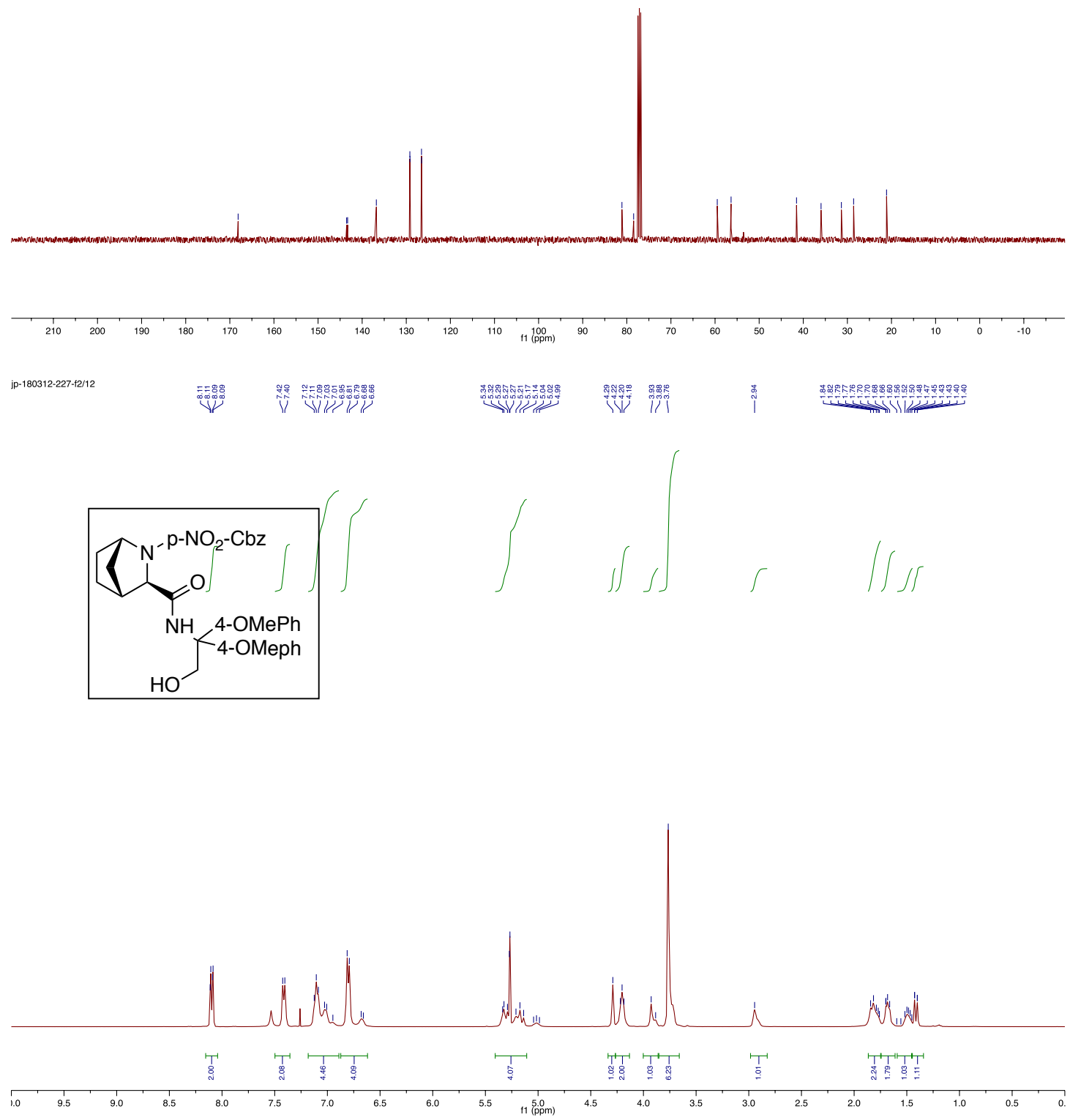
jp-180312-227--2/13
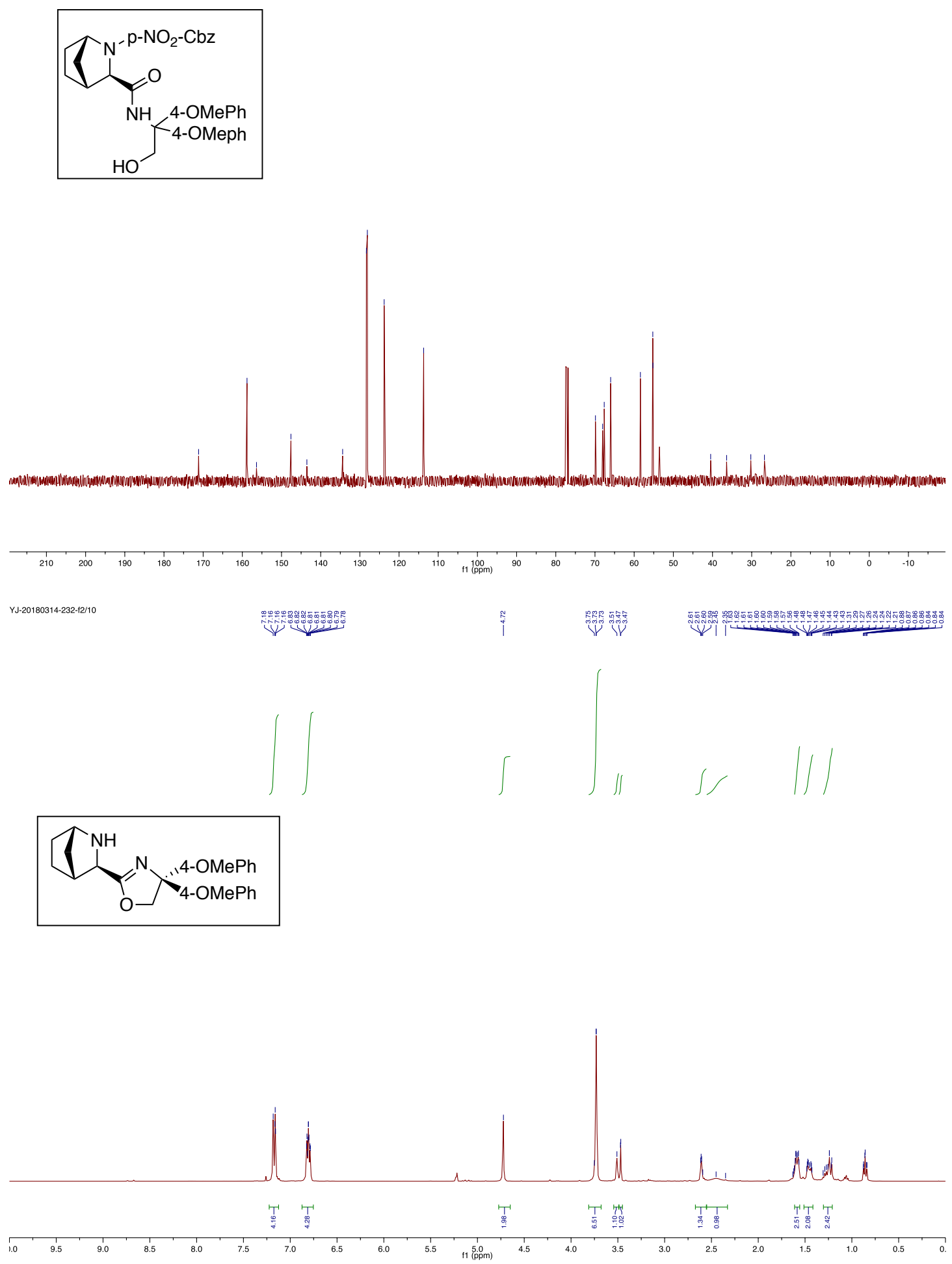
YJ-20180314-232-2/11

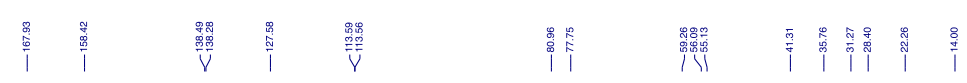

$$
\overbrace{0-14-O M e P h}^{N H}
$$
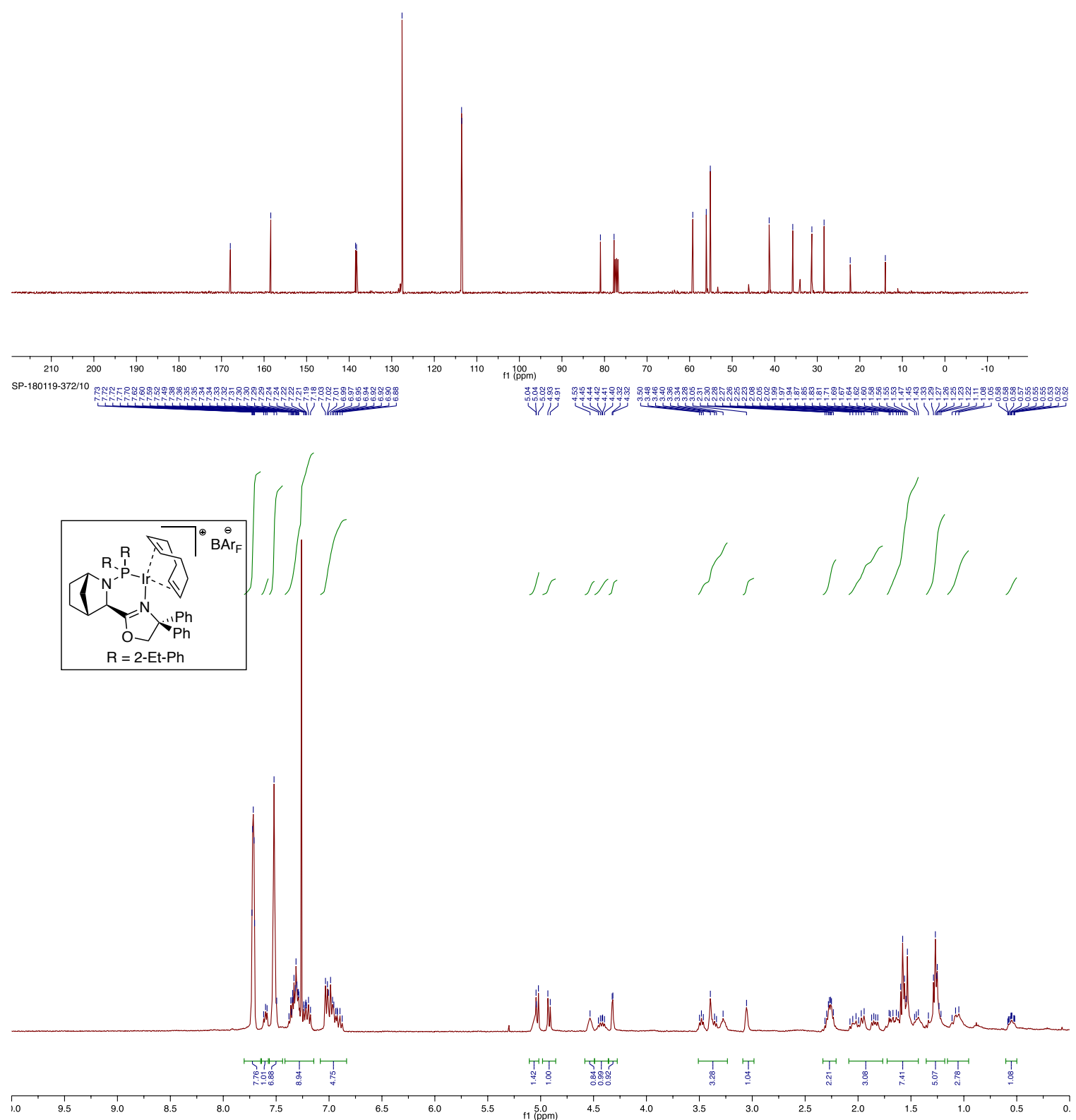

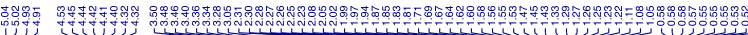



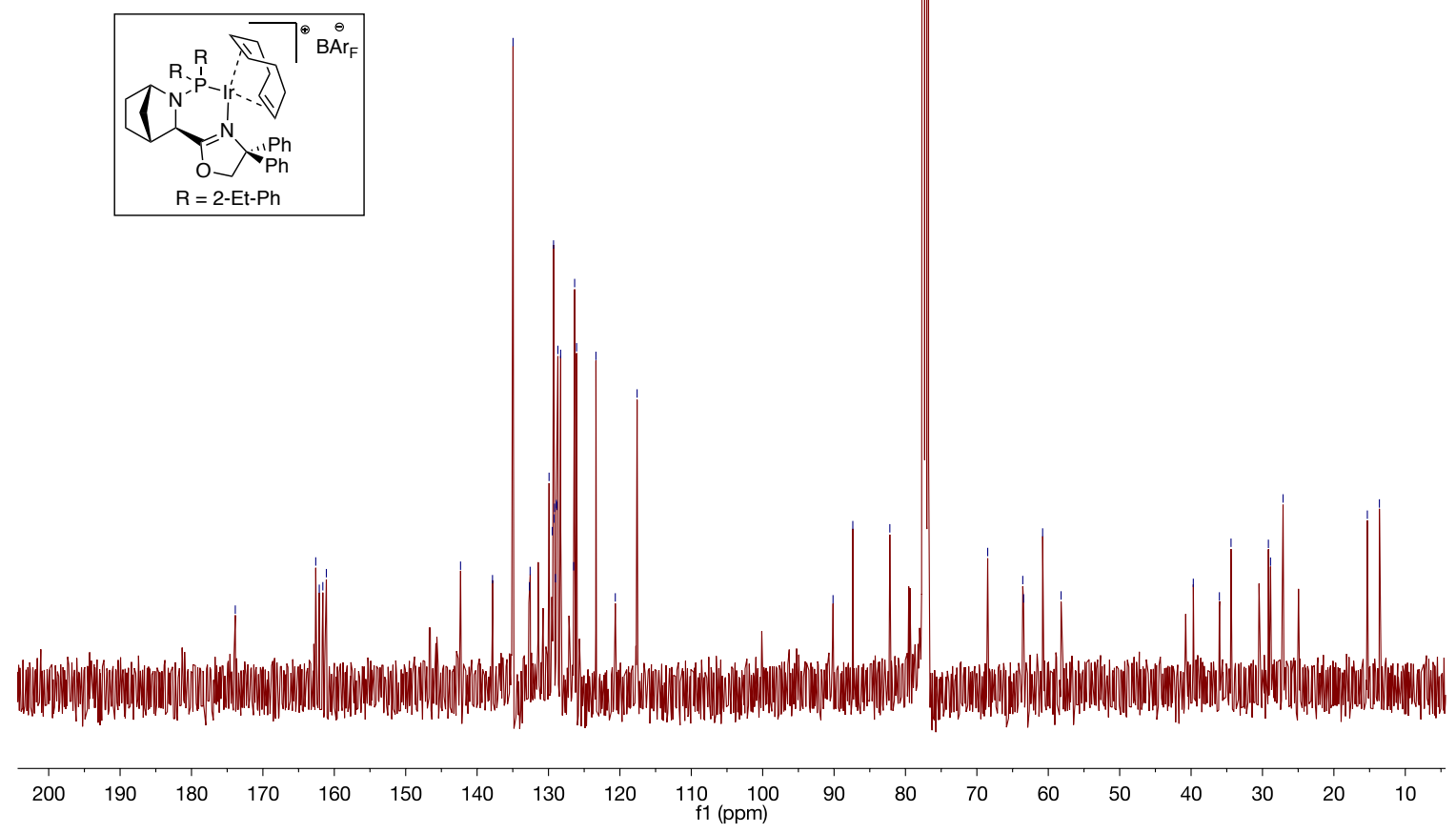

SP-180119-372/11

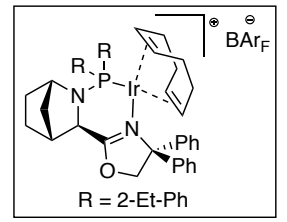

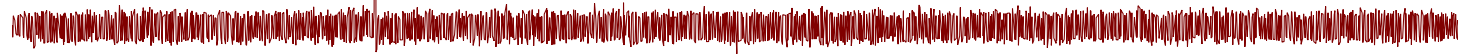

$\begin{array}{lllllllllllllllllllllll}140 & 120 & 100 & 80 & 60 & 40 & 20 & 0 & -20 & -40 & -60 & -80 & -100 & -120 & -140 & -160 & -180 & -200 & -220 & -240\end{array}$




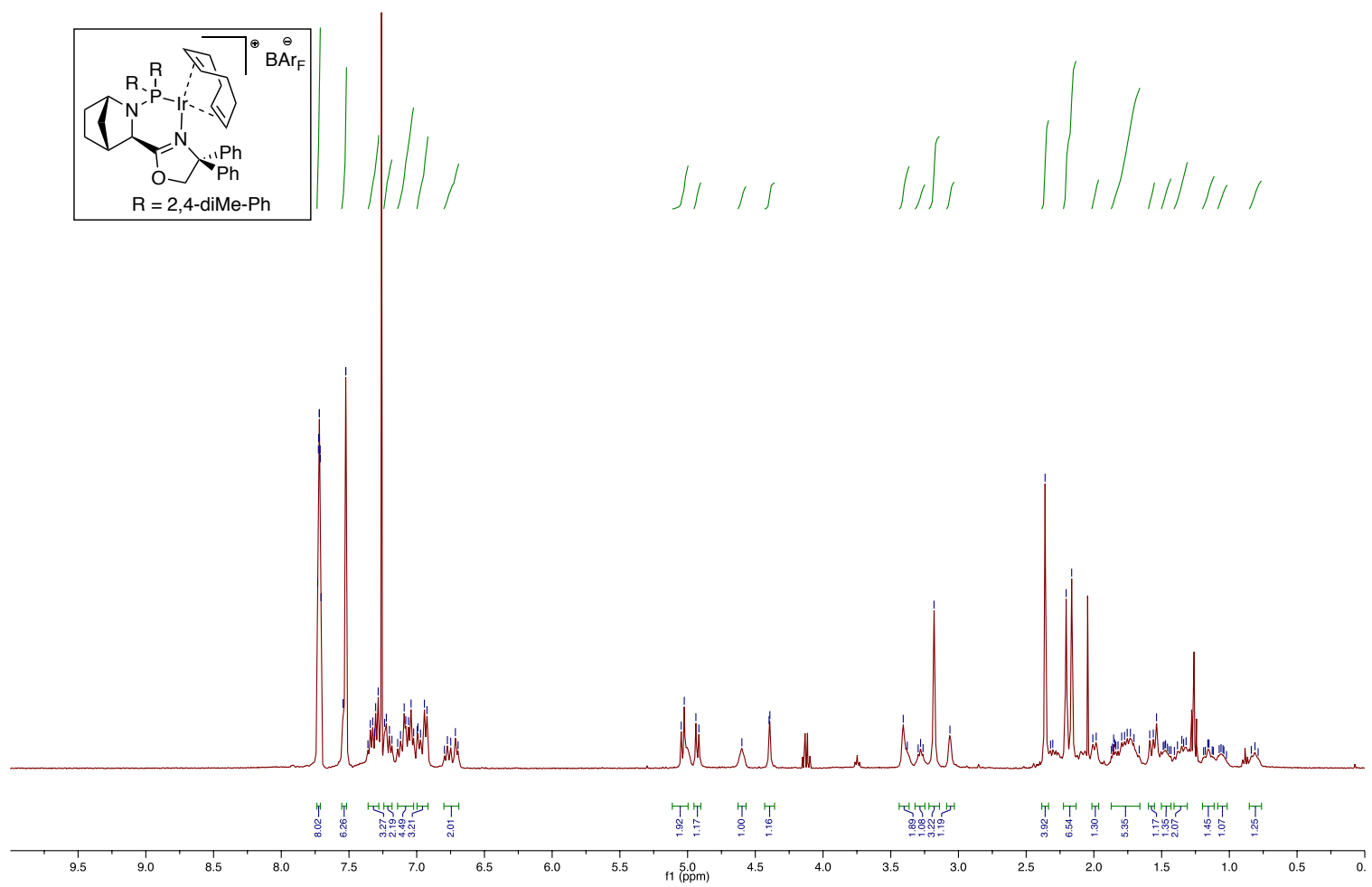

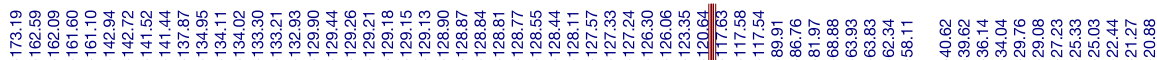

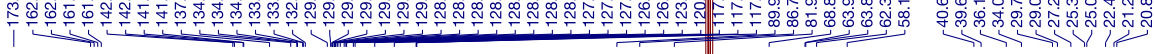

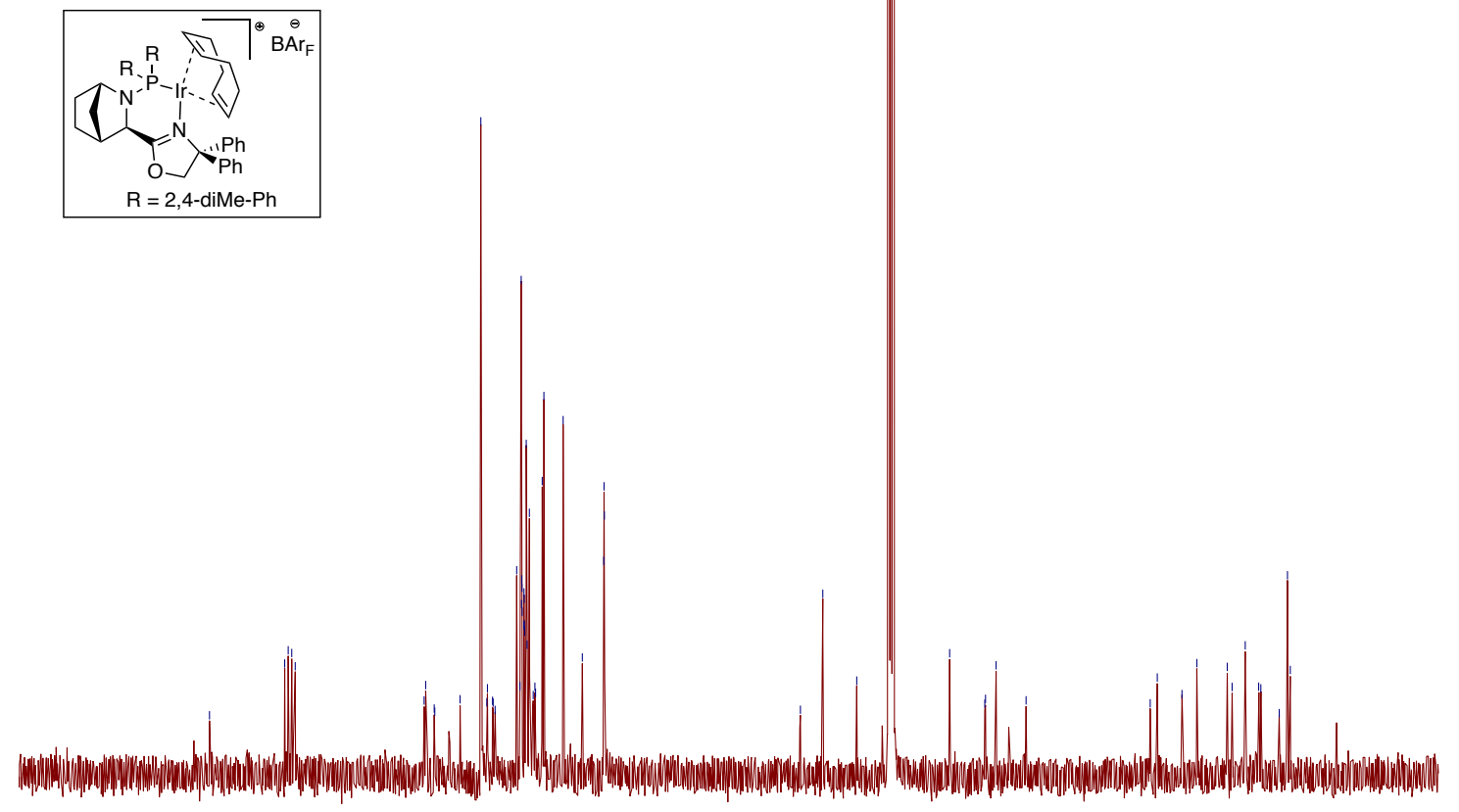

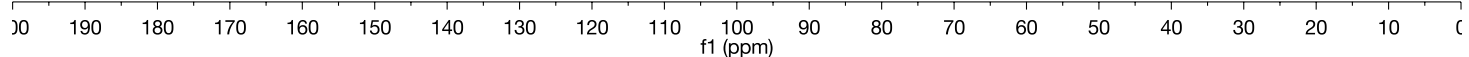




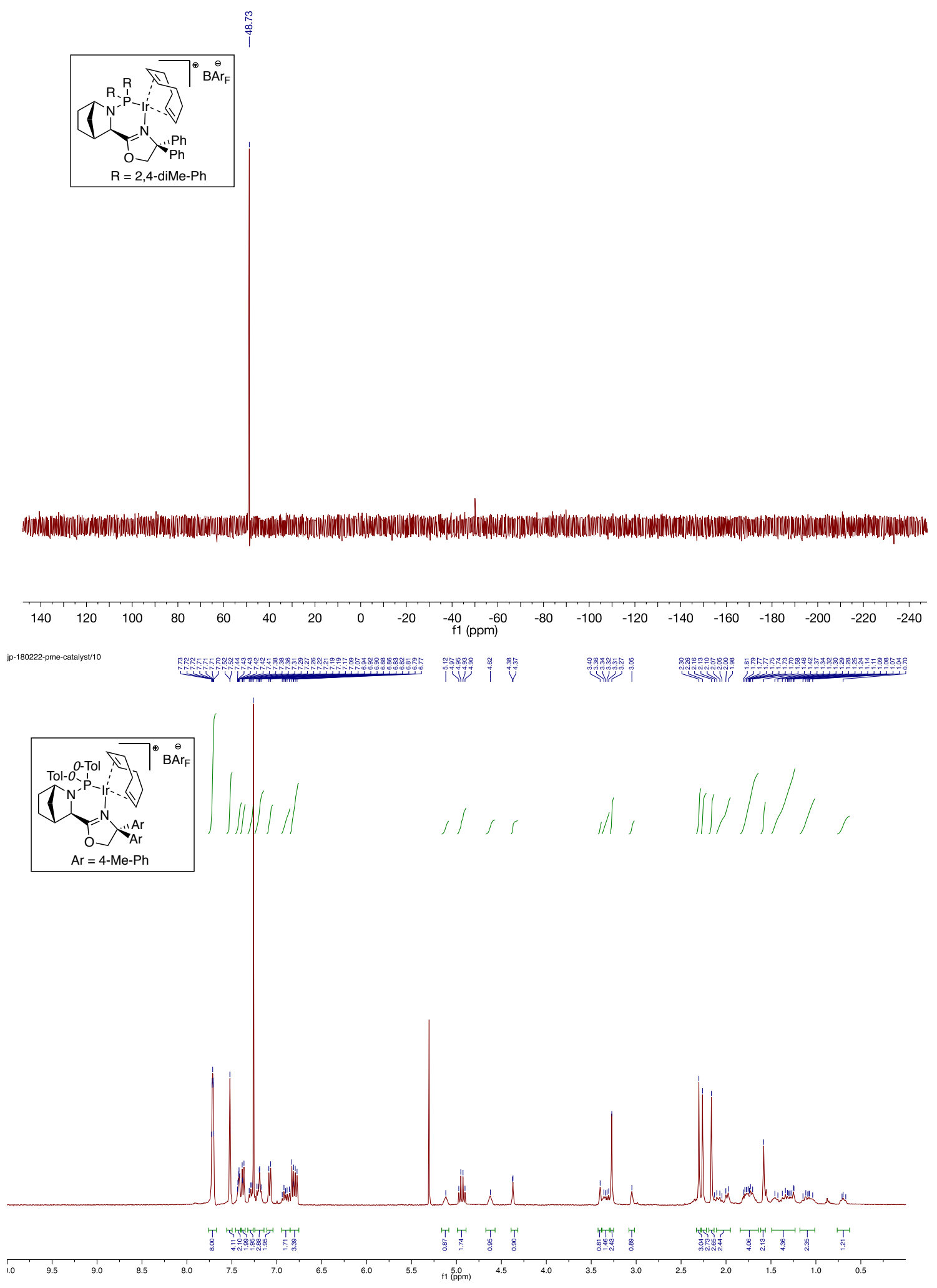

S-163 

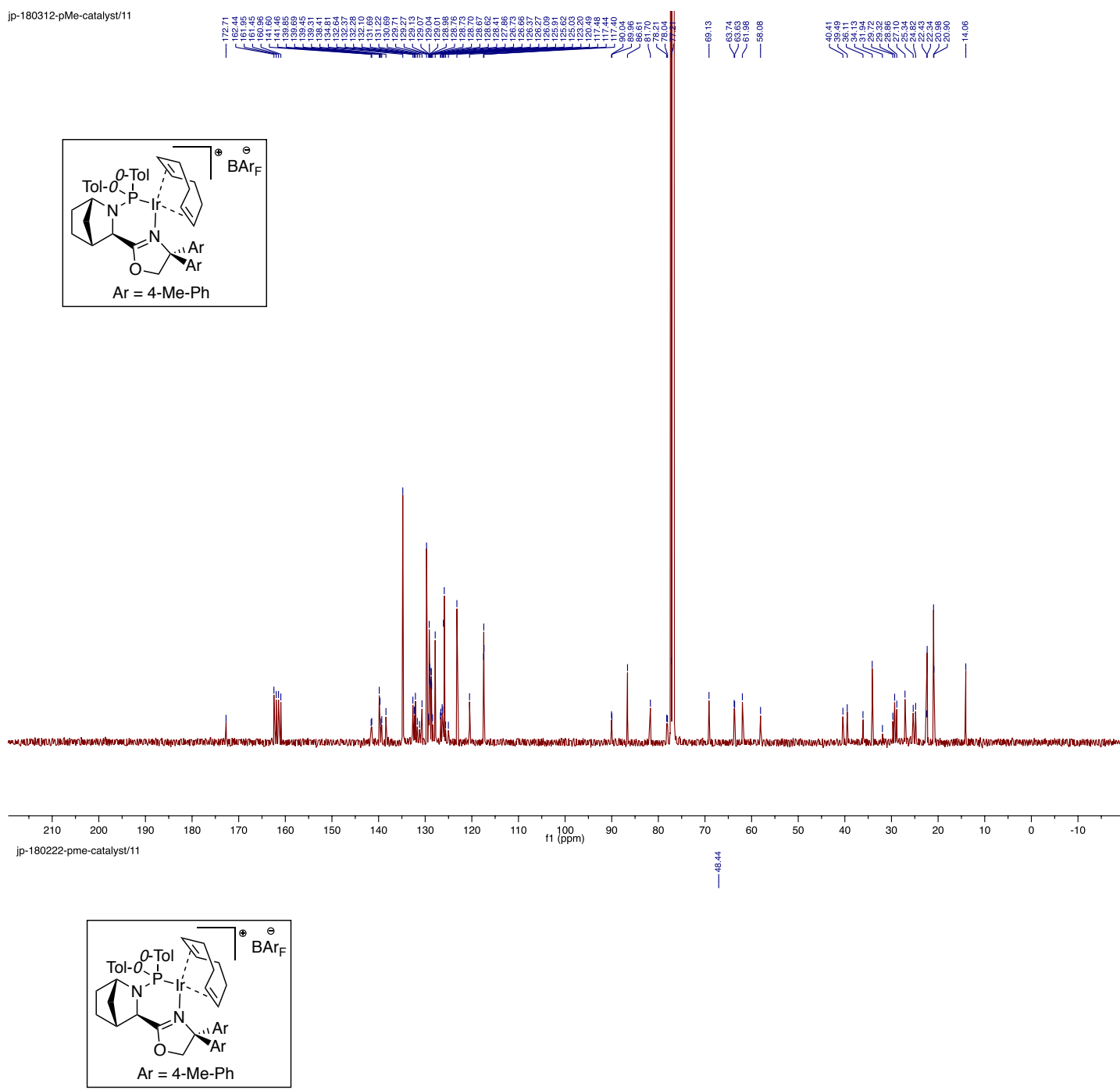



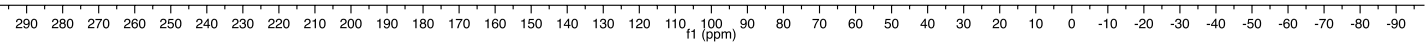



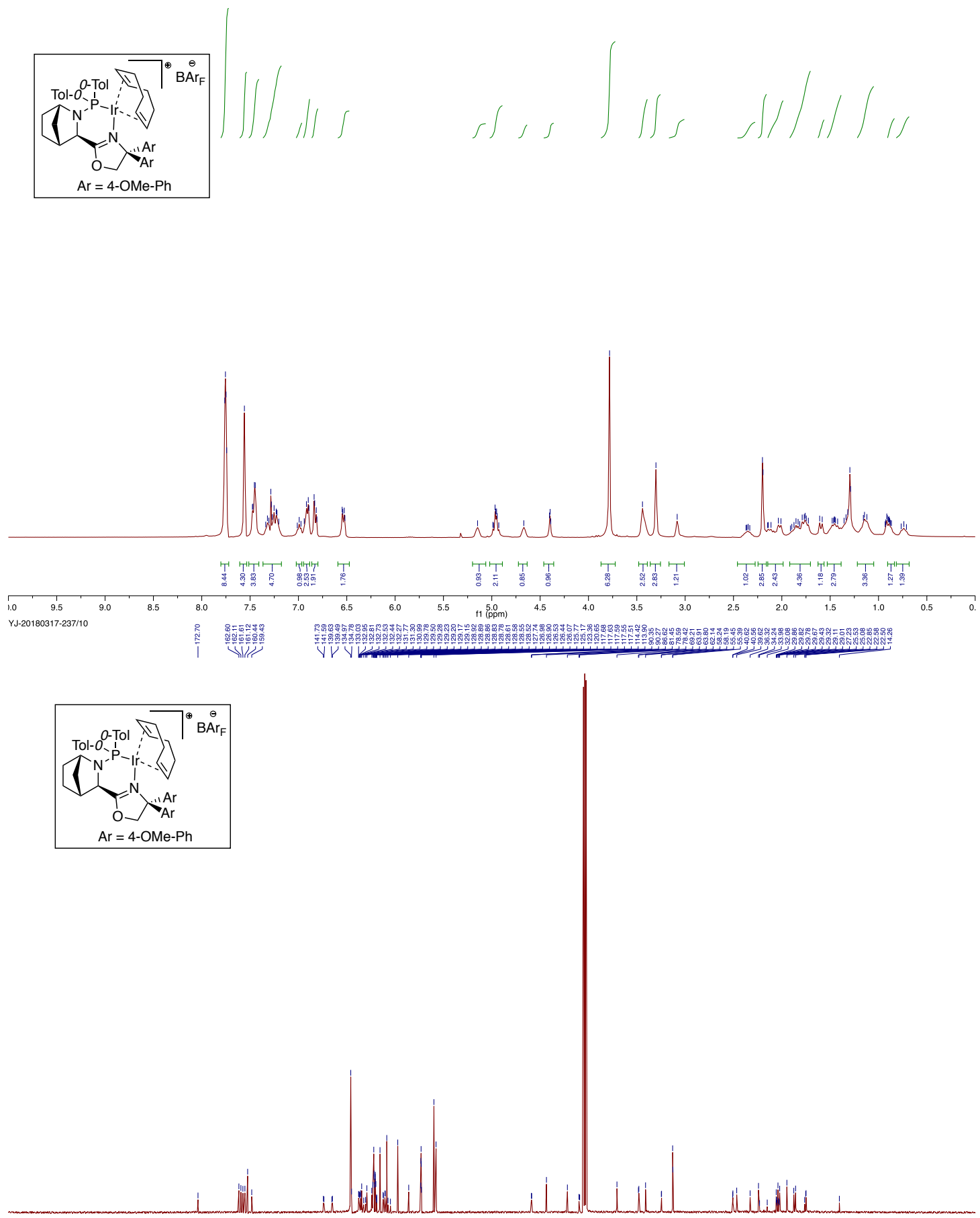


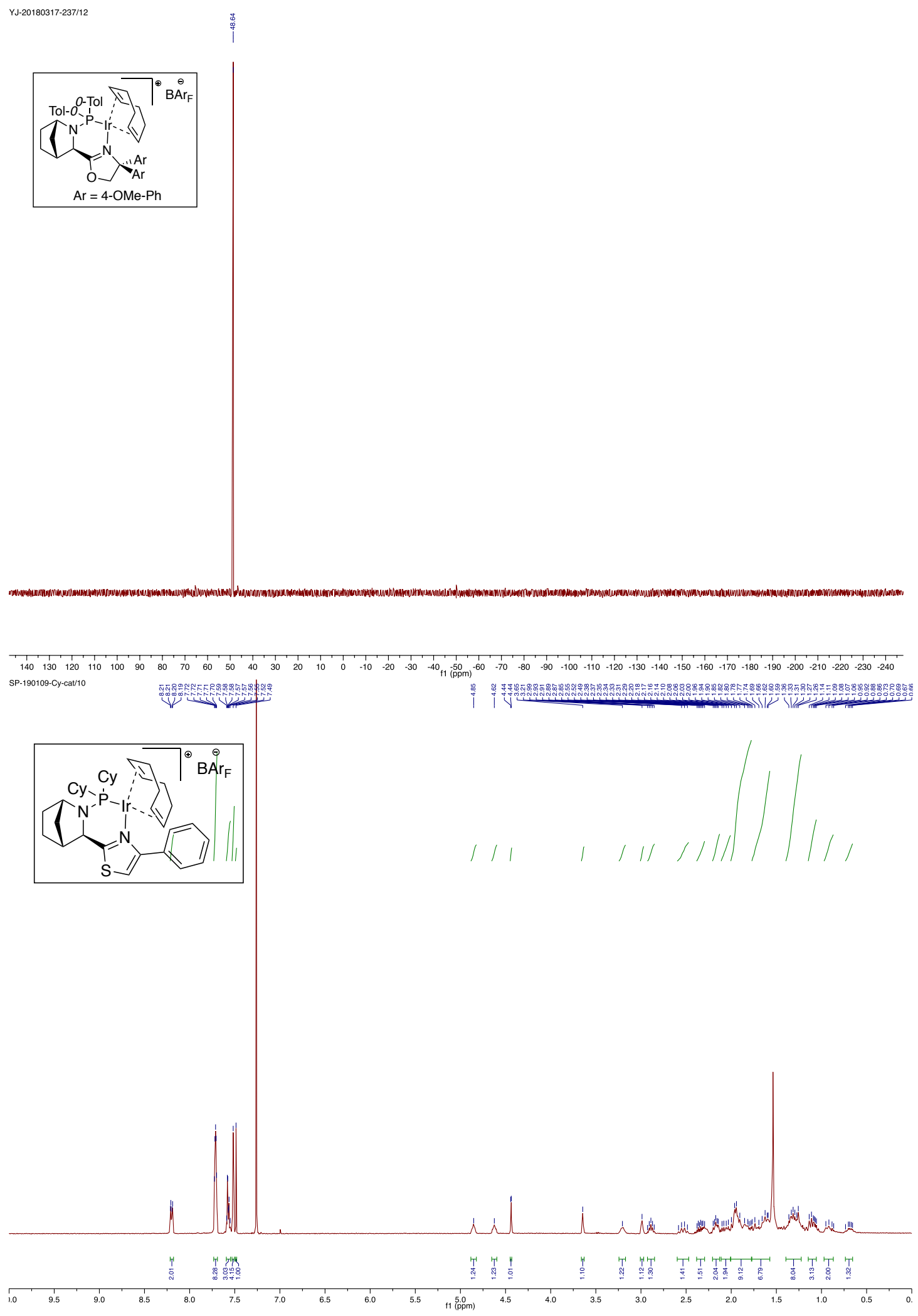



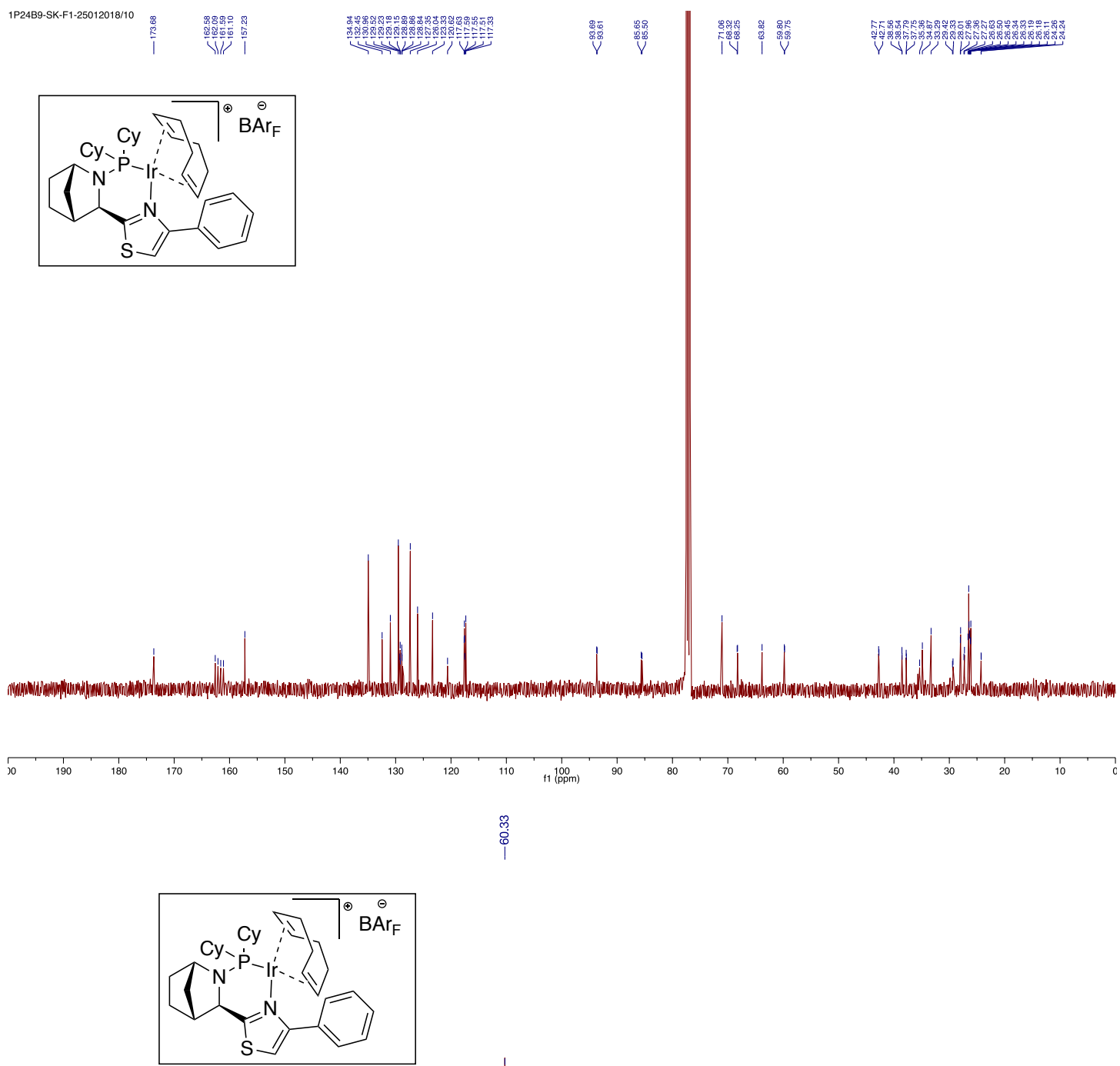

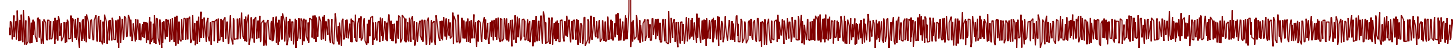

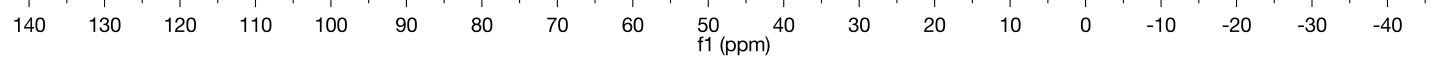




\section{References:}

1. Shen, X.-Y.; Peng, X.-S.; Wong, H. N.C. Total Synthesis of ( \pm -Gracilioether F. Org. Lett. 2016, 18, 1032-1035.

2. Okuro, K.; Alper, H. Palladium-catalyzed intermolecular cyclocarbonylation of 2iodoanilines with the Michael acceptor, diethyl ethoxycarbonylbutendienoate. J. Org. Chem. 2012, 77, 4420-4424.

3. Cserényi, S.; Felföldi, K.; Forgo, P.; Pálinko, I. Preparation of 3-substituted and 2, 3disubstituted-4, 4, 4-trifluoro-2-butenoic acids-Perkin condensation of activated aromatic ketones. J. Fluor. Chem., 2006, 127, 850-853.

4. Trifonova, A.; Diesen, J. S.; Chapman, C. J.; Andersson, P. G. Application of phosphine- oxazoline ligands in ir-catalyzed asymmetric hydrogenation of acyclic aromatic N-arylimines. Org. Lett. 2004, 6, 3825-3827. 